\title{
RESULTS OF OBSERVATIONS
}

\author{
OF THE \\ SUN, MOON, AND PLANETS \\ FROM 1913 TO 1925
}




\section{EXPLANATION}

In the following pages are given the final observed right ascensions and declinations of the Sun, Moon, and planets and the corresponding corrections to the American Ephemeris, or to other tabular places.

As already explained, besides the usual corrections for instrumental constants, pivots, clock corrections, motion, defection, and semidiameter, the right ascensions were corrected for personal equation (p. 33); semidiameter correction for the Moon (p. 66), day terms (p. 62); and equinox correction (p. 73).

And besides the usual corrections for instrumental constants, division errors, flexure, refraction, latitude, variation of latitude, clamp difference, motion, defection, semidiameter, and parallax; the declinations were corrected for day terms (p. 64); personal equations for the Sun (p. 49); semidiameter correction for the Moon (p. 66); and corrections to the provisional latitude, refraction, and flexure (p. 53).

Corrections to tabular places have been collected by years, or otherwise, and given here following the individual results.

102 


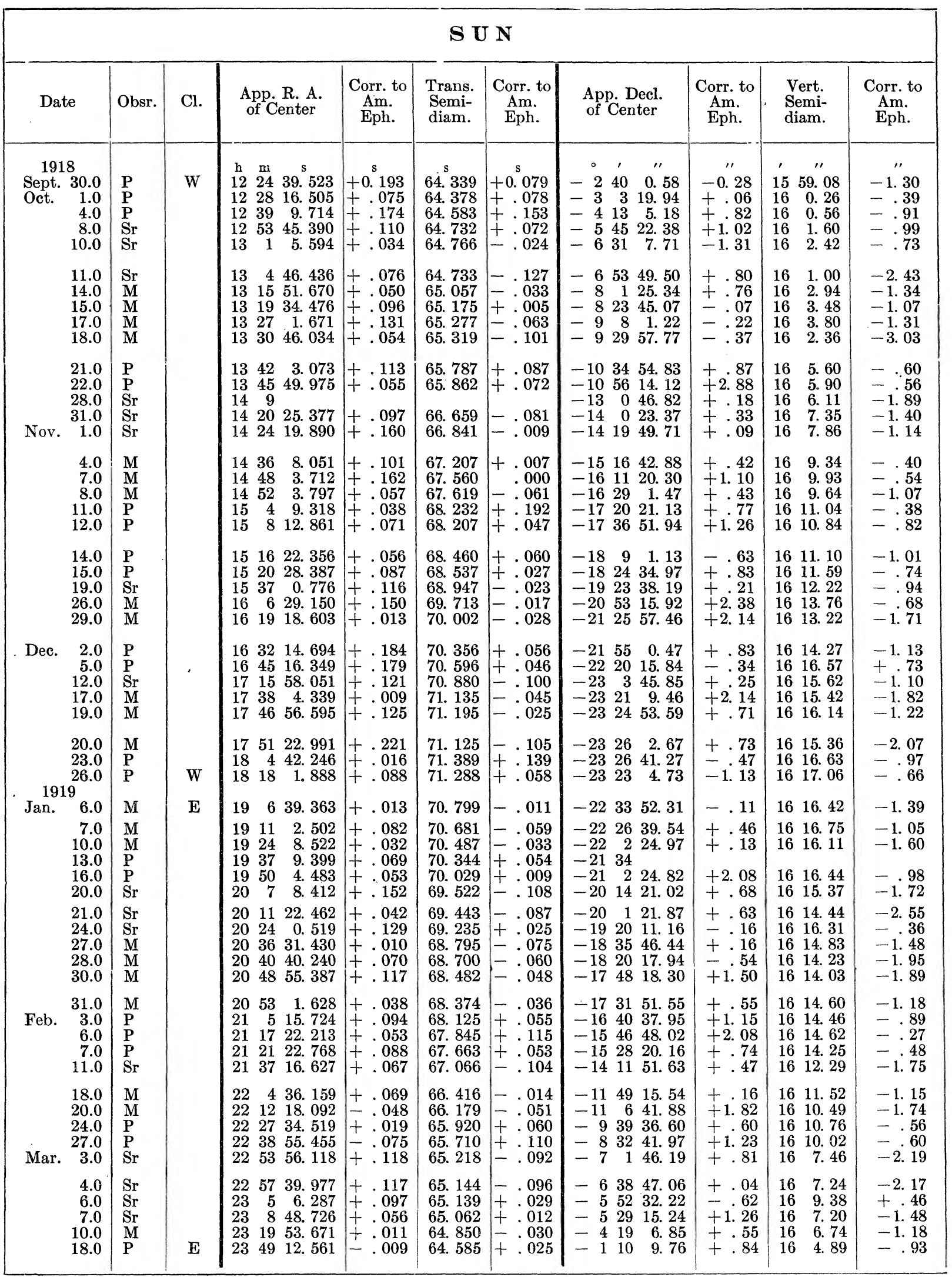




\begin{tabular}{|c|c|c|c|c|c|c|c|c|c|c|c|}
\hline \multicolumn{12}{|c|}{$\mathbf{S} \mathbf{U} \mathbf{N}$} \\
\hline $\mathrm{Da}$ & & Obsr. & Cl. & $\begin{array}{c}\text { App. R. A. } \\
\text { of Center }\end{array}$ & $\begin{array}{c}\text { Corr. to } \\
\text { Am. } \\
\text { Eph. }\end{array}$ & $\begin{array}{l}\text { Trans. } \\
\text { Semi- } \\
\text { diam. }\end{array}$ & $\begin{array}{c}\text { Corr. to } \\
\text { Am. } \\
\text { Eph. }\end{array}$ & $\begin{array}{l}\text { App. Decl. } \\
\text { of Center }\end{array}$ & $\begin{array}{l}\text { Corr. to } \\
\text { Am. } \\
\text { Eph. }\end{array}$ & $\begin{array}{l}\text { Vert. } \\
\text { Semi- } \\
\text { diam. }\end{array}$ & $\begin{array}{c}\text { Corr. to } \\
\text { Am. } \\
\text { Eph. }\end{array}$ \\
\hline $\begin{array}{l}19 \\
\text { Mar. } \\
\text { Apr. }\end{array}$ & $\begin{array}{r}19 \\
20.0 \\
21.0 \\
24.0 \\
25.0 \\
8.0\end{array}$ & $\begin{array}{l}\mathrm{P} \\
\mathrm{P} \\
\mathrm{Sr} \\
\mathrm{Sr} \\
\mathrm{P}\end{array}$ & $\underset{\mathrm{W}}{\mathrm{E}}$ & $\begin{array}{rrr}\mathrm{h} & \mathrm{m} & \mathrm{s} \\
23 & 56 & 30.103 \\
0 & 0 & 8.652 \\
0 & 11 & 3.659 \\
0 & 14 & 41.832 \\
1 & 6 & \end{array}$ & $\begin{array}{c}\mathrm{s} \\
+0.053 \\
+.082 \\
+.089 \\
+.052\end{array}$ & $\begin{array}{l}\text { s } \\
64.573 \\
64.576 \\
64.419 \\
64.418\end{array}$ & $\begin{array}{c}\mathrm{s} \\
+0.053 \\
+.076 \\
-.031 \\
-.022\end{array}$ & 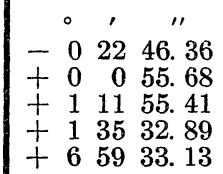 & $\begin{array}{c}\prime \prime \\
-0.26 \\
-.02 \\
+.01 \\
+.69 \\
+.43\end{array}$ & \begin{tabular}{|cr}
$\prime$ & \multicolumn{1}{c}{${ }^{\prime \prime}$} \\
16 & 3.50 \\
16 & 5.39 \\
16 & 4.09 \\
16 & 3.52 \\
15 & 59.24
\end{tabular} & $\begin{array}{r}\prime \prime \\
-1.77 \\
+.39 \\
-.07 \\
-.36 \\
-.78\end{array}$ \\
\hline May & $\begin{array}{r}18.0 \\
21.0 \\
22.0 \\
29.0 \\
2.0\end{array}$ & $\begin{array}{l}\mathrm{Sr} \\
\mathrm{M} \\
\mathrm{M} \\
\mathrm{P} \\
\mathrm{P}\end{array}$ & & $\begin{array}{lll}1 & 42 & 31.065 \\
1 & 53 & 40.608 \\
1 & 57 & 24.755 \\
2 & 23 & 46.825 \\
2 & 35 & 12.474\end{array}$ & $\begin{array}{r}+.055 \\
+.008 \\
+.085 \\
+.075 \\
+.016\end{array}$ & $\begin{array}{l}\text { 65. } 048 \\
65.346 \\
65.335 \\
65.820 \\
66.264\end{array}$ & $\begin{array}{l}-.052 \\
+.066 \\
-.005 \\
\bar{t} .020 \\
+.194\end{array}$ & 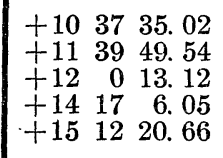 & $\begin{array}{l}+.82 \\
+.36 \\
+.32 \\
+.45 \\
+.16\end{array}$ & $\begin{array}{ll}15 & 57.40 \\
15 & 55.76 \\
15 & 55.16 \\
15 & 53.16 \\
15 & 54.86\end{array}$ & $\begin{array}{l}+.05 \\
-.79 \\
-1.12 \\
-1.35 \\
+1.07\end{array}$ \\
\hline & $\begin{array}{r}5.0 \\
12.0 \\
13.0 \\
15.0 \\
19.0\end{array}$ & $\begin{array}{l}\mathrm{Sr} \\
\mathbf{M} \\
\mathrm{M} \\
\mathbf{M} \\
\mathrm{P}\end{array}$ & & $\begin{array}{lll}2 & 46 & 43.000 \\
3 & 13 & 53.374 \\
3 & 18 & \\
3 & 25 & 40 . \\
3 & 41 & 31.361\end{array}$ & $\begin{array}{l}+.010 \\
+.074 \\
+.023 \\
+.021\end{array}$ & $\begin{array}{l}66.265 \\
66.883 \\
67.108 \\
67.505\end{array}$ & $\begin{array}{l}-.045 \\
+.003 \\
-.022 \\
+.055\end{array}$ & $\begin{array}{rrr}+16 & 5 & 20.20 \\
+17 & 59 & 22.11 \\
+18 & 14 & 30.60 \\
+18 & 43 & 48.96 \\
+19 & 38 & 39.33\end{array}$ & $\begin{array}{l}+.60 \\
+.01 \\
+1.10 \\
+.04 \\
+.43\end{array}$ & $\begin{array}{ll}15 & 51.64 \\
15 & 50.94 \\
15 & 50.96 \\
15 & 49.98 \\
15 & 49.54\end{array}$ & $\begin{array}{l}-1.46 \\
=.67 \\
-.45 \\
-1.02 \\
-.67\end{array}$ \\
\hline & $\begin{array}{l}22.0 \\
23.0 \\
26.0 \\
27.0 \\
29.0\end{array}$ & $\begin{array}{l}\mathrm{P} \\
\mathrm{P} \\
\mathrm{Sr} \\
\mathrm{Sr} \\
\mathrm{Sr}\end{array}$ & & $\begin{array}{rrr}3 & 53 & 30.550 \\
3 & 57 & 31.350 \\
4 & 9 & 37.137 \\
4 & 13 & 40.100 \\
4 & 21 & 47.436\end{array}$ & $\begin{array}{r}+.060 \\
+.020 \\
+.067 \\
+.070 \\
+.036\end{array}$ & $\begin{array}{l}67.702 \\
67.823 \\
67.884 \\
68.020 \\
68.080\end{array}$ & $\begin{array}{l}+.022 \\
+.073 \\
-.076 \\
-.010 \\
-.080\end{array}$ & $\begin{array}{rrr}+20 & 16 & 17.91 \\
+20 & 28 & 9.82 \\
+21 & 1 & 37.47 \\
+21 & 12 & 4.13 \\
+21 & 31 & 50.22\end{array}$ & $\begin{array}{l}+.41 \\
+.52 \\
+.07 \\
+.53 \\
+.12\end{array}$ & $\begin{array}{ll}15 & 49.84 \\
15 & 49.32 \\
15 & 47.24 \\
15 & 47.82 \\
15 & 47.27\end{array}$ & $\begin{array}{l}+.20 \\
=.14 \\
-1.70 \\
=.96 \\
-1.20\end{array}$ \\
\hline June & $\begin{array}{r}2.0 \\
3.0 \\
13.0 \\
16.0 \\
17.0\end{array}$ & $\begin{array}{l}\mathrm{M} \\
\mathrm{M} \\
\mathrm{P} \\
\mathrm{Sr} \\
\mathrm{Sr}\end{array}$ & & $\begin{array}{rrr}4 & 38 & 7.564 \\
4 & 42 & 13.484 \\
5 & 23 & 30.304 \\
5 & 35 & 57.526 \\
5 & 40 & 7.019\end{array}$ & $\begin{array}{r}+.114 \\
+.034 \\
+.094 \\
+.006 \\
+.129\end{array}$ & $\begin{array}{l}68.335 \\
68.360 \\
68.962 \\
68.805 \\
68.841\end{array}$ & $\begin{array}{l}-.065 \\
-.100 \\
+.112 \\
-.105 \\
-.079\end{array}$ & $\begin{array}{rrr}+22 & 6 & 53.53 \\
+22 & 14 & 41.61 \\
+23 & 11 & 0.90 \\
+23 & 20 & 0.71 \\
+23 & 22 & 13.34\end{array}$ & $\begin{array}{l}+.63 \\
+.31 \\
+.60 \\
+.19 \\
+1.54\end{array}$ & $\begin{array}{ll}15 & 46.17 \\
15 & 46.36 \\
15 & 46.56 \\
15 & 44.67 \\
15 & 46.42\end{array}$ & $\begin{array}{l}-1.73 \\
-1.41 \\
-.13 \\
-1.76 \\
+.07\end{array}$ \\
\hline July & $\begin{array}{r}19.0 \\
20.0 \\
23.0 \\
30.0 \\
1.0\end{array}$ & $\begin{array}{l}\mathrm{Sr} \\
\mathrm{Sr} \\
\mathrm{Sr} \\
\mathrm{Sr} \\
\mathrm{P}\end{array}$ & & $\begin{array}{rrr}5 & 48 & 25.967 \\
5 & 52 & 35.567 \\
6 & 5 & 4.458 \\
6 & 34 & 9.224 \\
6 & 38 & 17.815\end{array}$ & $\begin{array}{l}+.057 \\
+.047 \\
+.018 \\
-.096 \\
-.065\end{array}$ & $\begin{array}{l}68.898 \\
68.849 \\
68.890 \\
68.780 \\
68.865\end{array}$ & $\begin{array}{l}-.042 \\
-.091 \\
-.050 \\
.010 \\
+.105\end{array}$ & $\begin{array}{rrr}+23 & 25 & 20.94 \\
+23 & 26 & 16.77 \\
+23 & 26 & 36.60 \\
+23 & 12 & 57.82 \\
+23 & 9 & 23.65\end{array}$ & $\begin{array}{l}+1.64 \\
+.87 \\
+.20 \\
+.18 \\
+.85\end{array}$ & $\begin{array}{ll}15 & 45.00 \\
15 & 44.23 \\
15 & 44.62 \\
15 & 44.66 \\
15 & 44.91\end{array}$ & $\begin{array}{l}-1.21 \\
-1.91 \\
-1.33 \\
-1.05 \\
-.79\end{array}$ \\
\hline Aug. & $\begin{array}{r}3.0 \\
4.0 \\
7.0 \\
8.0 \\
11.0\end{array}$ & $\begin{array}{l}\mathrm{P} \\
\mathrm{Sr} \\
\mathrm{Sr} \\
\mathrm{Sr} \\
\mathrm{M}\end{array}$ & & $\begin{array}{rrr}6 & 46 & 34.265 \\
8 & 54 & 52.272 \\
9 & 6 & 24.110 \\
9 & 10 & 13.538 \\
9 & 21 & 38.165\end{array}$ & $\begin{array}{r}+.075 \\
+.092 \\
+.060 \\
+.088 \\
+.085\end{array}$ & $\begin{array}{l}\text { 68. } 723 \\
66.345 \\
66.069 \\
\text { 65. } 988 \\
\text { 65. } 743\end{array}$ & $\begin{array}{l}+.033 \\
-.045 \\
-.071 \\
-.062 \\
-.057\end{array}$ & $\begin{array}{rrr}+23 & 0 & 59.27 \\
+17 & 24 & 21.16 \\
+16 & 35 & 43.44 \\
+16 & 18 & 58.01 \\
+15 & 27 & 9.06\end{array}$ & $\begin{array}{l}-.53 \\
+.26 \\
+.74 \\
+.51 \\
+.06\end{array}$ & $\begin{array}{ll}15 & 45.00 \\
15 & 46.34 \\
15 & 46.76 \\
15 & 46.83 \\
15 & 47.30\end{array}$ & $\begin{array}{l}-.69 \\
-1.47 \\
-1.48 \\
-1.56 \\
-1.55\end{array}$ \\
\hline & $\begin{array}{l}12.0 \\
14.0 \\
15.0 \\
18.0 \\
19.0\end{array}$ & $\begin{array}{l}\mathbf{M} \\
\mathbf{M} \\
\mathbf{M} \\
\mathbf{M} \\
\mathbf{M}\end{array}$ & & $\begin{array}{lll}9 & 25 & 25.239 \\
9 & 32 & 57.684 \\
9 & 36 & 43.125 \\
9 & 47 & 56.248 \\
9 & 51 & 39.674\end{array}$ & $\begin{array}{l}+.099 \\
+.104 \\
+.125 \\
+.078 \\
+.114\end{array}$ & $\begin{array}{l}\text { 65. } 648 \\
65.514 \\
65.452 \\
65.205 \\
65.122\end{array}$ & $\begin{array}{l}-.072 \\
-.036 \\
-.018 \\
-.045 \\
-.058\end{array}$ & $\begin{array}{rrr}+15 & 9 & 22.13 \\
+14 & 33 & 7.71 \\
+14 & 14 & 38.08 \\
+13 & 17 & 49.61 \\
+12 & 58 & 27.89\end{array}$ & $\begin{array}{l}-.67 \\
+.81 \\
+.28 \\
+.01 \\
+.29\end{array}$ & $\begin{array}{ll}15 & 47.47 \\
15 & 48.34 \\
15 & 48.46 \\
15 & 49.18 \\
15 & 48.89\end{array}$ & $\begin{array}{l}-1.54 \\
-1.01 \\
-1.06 \\
-. .85 \\
-1.32\end{array}$ \\
\hline Sept. & $\begin{array}{r}21.0 \\
22.0 \\
26.0 \\
8.0 \\
11.0\end{array}$ & $\begin{array}{l}\mathrm{P} \\
\mathrm{P} \\
\mathrm{P} \\
\mathrm{M} \\
\mathrm{M}\end{array}$ & & $\begin{array}{rrr}9 & 59 & 5.076 \\
10 & 2 & 47.064 \\
10 & 18 & \\
11 & 4 & 42.185 \\
11 & 15 & 29.613\end{array}$ & $\begin{array}{l}+.126 \\
+.124 \\
+.025 \\
+.113\end{array}$ & $\begin{array}{l}65.057 \\
64.996 \\
\\
64.106 \\
64.009\end{array}$ & $\begin{array}{l}+.017 \\
+.026 \\
-.034 \\
-.061\end{array}$ & $\begin{array}{rrr}+12 & 19 & 6.71 \\
+11 & 59 & 8.07 \\
+10 & 37 & 23.64 \\
+\quad 5 & 55 & 1.96 \\
+ & 447 & 7.06\end{array}$ & $\begin{array}{l}+.11 \\
-.23 \\
=.56 \\
\overline{.} .34 \\
+.96\end{array}$ & $\begin{array}{ll}15 & 49.47 \\
15 & 51.07 \\
15 & 50.86 \\
15 & 53.26 \\
15 & 54.22\end{array}$ & $\begin{array}{l}-1.11 \\
+.30 \\
-.72 \\
-1.33 \\
-1.11\end{array}$ \\
\hline & $\begin{array}{l}12.0 \\
15.0 \\
16.0 \\
18.0 \\
25.0\end{array}$ & $\begin{array}{l}\mathbf{M} \\
\mathbf{P} \\
\mathbf{P} \\
\mathbf{P} \\
\mathbf{M}\end{array}$ & & $\begin{array}{rrr}11 & 19 & 5.102 \\
11 & 29 & 51.089 \\
11 & 33 & 26.399 \\
11 & 40 & 36.866 \\
12 & 5 & 45.504\end{array}$ & $\begin{array}{l}+.092 \\
+.049 \\
+.099 \\
+.066 \\
+.094\end{array}$ & $\begin{array}{l}64.028 \\
64.144 \\
64.087 \\
64.089 \\
64.063\end{array}$ & $\begin{array}{r}-.032 \\
+.124 \\
+.077 \\
+.079 \\
-.027\end{array}$ & $\begin{array}{rrrr}+ & 4 & 24 & 17.34 \\
+ & 3 & 15 & 23.31 \\
+ & 2 & 52 & 16.36 \\
+ & 2 & 5 & 56.25 \\
- & 0 & 37 & 28.19\end{array}$ & $\begin{array}{l}+.24 \\
+.41 \\
.64 \\
+.95 \\
-.99\end{array}$ & $\begin{array}{ll}15 & 54.56 \\
15 & 55.98 \\
15 & 55.99 \\
15 & 56.52 \\
15 & 57.38\end{array}$ & $\begin{array}{l}-1.02 \\
=.35 \\
=.59 \\
-.57 \\
-1.56\end{array}$ \\
\hline Oct. & $\begin{array}{r}26.0 \\
7.0 \\
10.0 \\
13.0 \\
16.0\end{array}$ & $\begin{array}{l}\mathrm{M} \\
\mathrm{M} \\
\mathrm{M} \\
\mathrm{P} \\
\mathrm{P}\end{array}$ & $\begin{array}{l}\mathrm{W} \\
\mathrm{E}\end{array}$ & 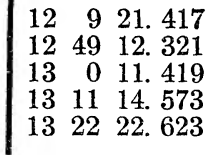 & $\begin{array}{l}+.037 \\
+.011 \\
+.069 \\
+.023 \\
+.03\end{array}$ & $\begin{array}{l}64.129 \\
64.646 \\
64.710 \\
65.115 \\
65.231\end{array}$ & $\begin{array}{r}+.009 \\
+.056 \\
-.060 \\
+.125 \\
+\quad .001\end{array}$ & 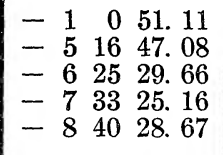 & $\begin{array}{l}+.09 \\
\pm .98 \\
-.76 \\
+.94 \\
-.37\end{array}$ & $\begin{array}{ll}15 & 58.77 \\
16 & 1.34 \\
16 & 1.32 \\
16 & 4.25 \\
16 & 3.22\end{array}$ & $\begin{array}{l}-.44 \\
-.96 \\
-1.81 \\
+.30 \\
-1.53\end{array}$ \\
\hline
\end{tabular}




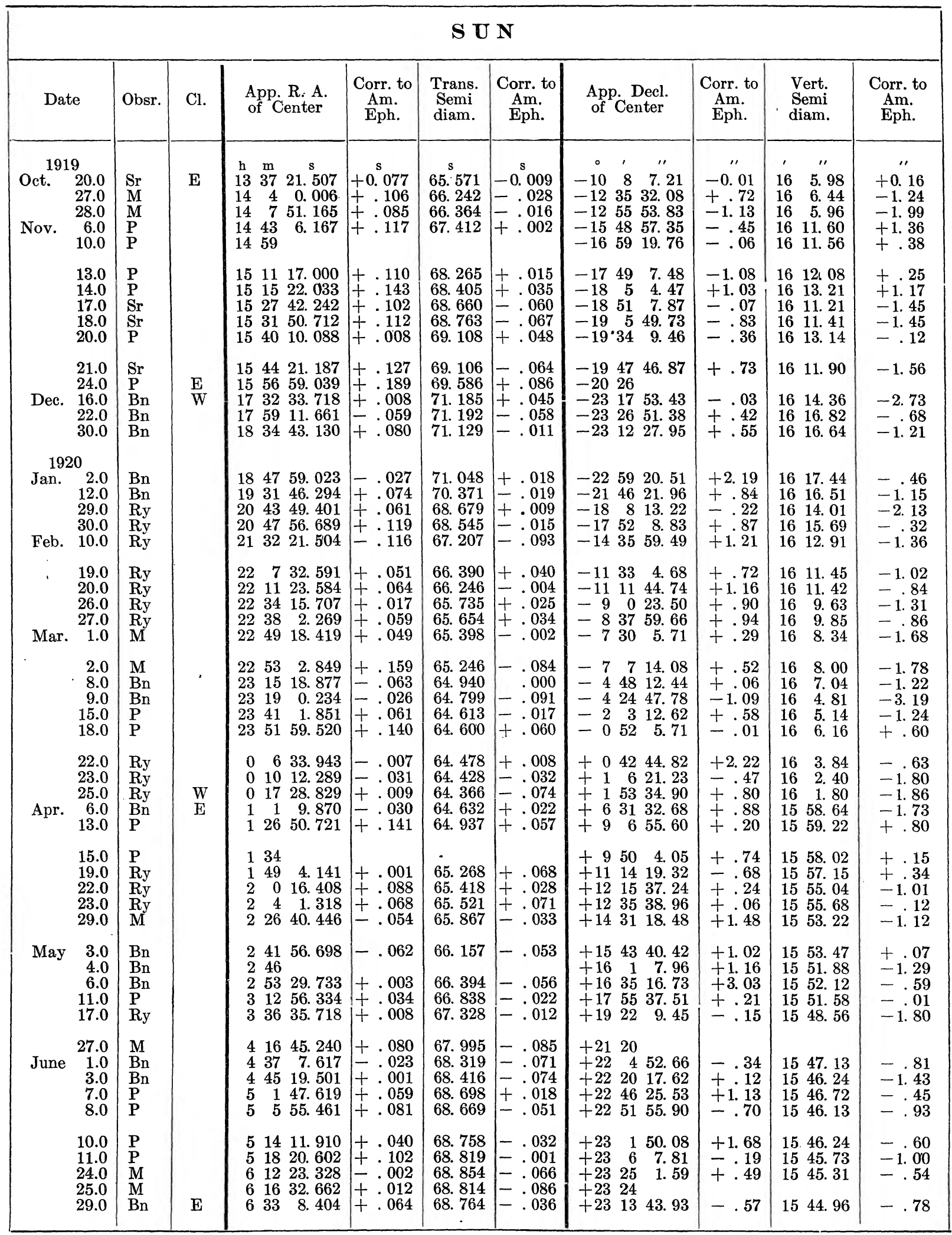




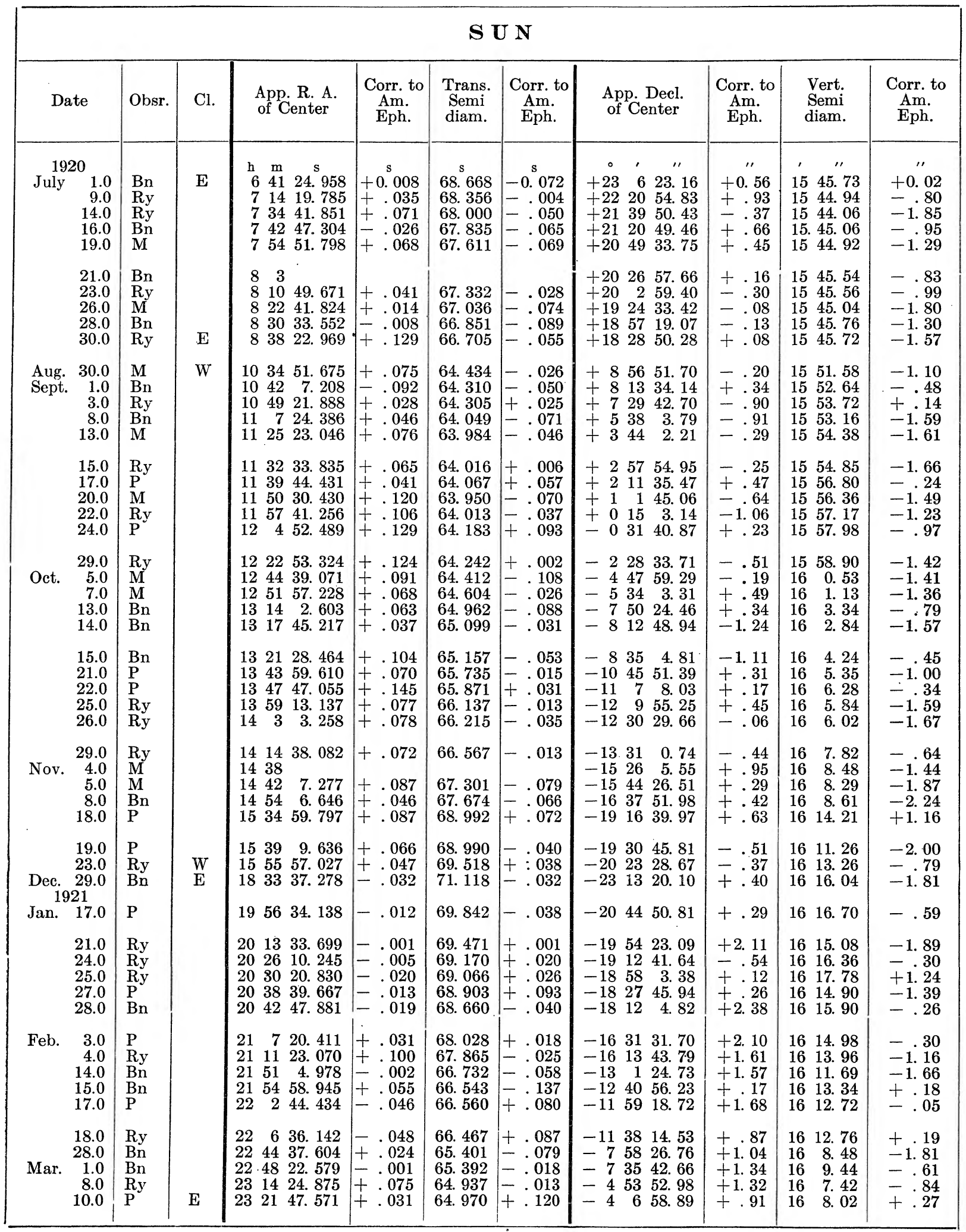




\begin{tabular}{|c|c|c|c|c|c|c|c|c|c|c|c|}
\hline \multicolumn{12}{|c|}{$\mathbf{S} \mathbf{U} \mathbf{N}$} \\
\hline Date & & Obsr. & Cl. & $\begin{array}{l}\text { App. R. A. } \\
\text { of Center }\end{array}$ & $\begin{array}{c}\text { Corr. to } \\
\text { Am. } \\
\text { Eph. }\end{array}$ & $\begin{array}{l}\text { Trans. } \\
\text { Semi } \\
\text { diam. }\end{array}$ & $\begin{array}{c}\text { Corr. to } \\
\text { Am. } \\
\text { Eph. }\end{array}$ & $\begin{array}{l}\text { App. Decl. } \\
\text { of Center }\end{array}$ & $\begin{array}{c}\text { Corr. to } \\
\text { Am. } \\
\text { Eph. }\end{array}$ & $\begin{array}{l}\text { Vert. } \\
\text { Semi } \\
\text { diam. }\end{array}$ & $\begin{array}{c}\text { Corr. to } \\
\text { Am. } \\
\text { Eph. }\end{array}$ \\
\hline $\begin{array}{r}1921 \\
\text { Mar. } 1 \\
1 \\
1 \\
1 \\
1\end{array}$ & $\begin{array}{l}1 \\
11.0 \\
14.0 \\
15.0 \\
17.0 \\
18.0\end{array}$ & $\begin{array}{l}\mathrm{Bn} \\
\mathrm{Bn} \\
\mathrm{Bn} \\
\mathrm{P} \\
\mathrm{Ry}\end{array}$ & $\mathbf{E}$ & \begin{tabular}{ccr|}
$\mathrm{h}$ & $\mathrm{m}$ & \multicolumn{1}{c}{$\mathrm{s}$} \\
23 & 25 & 28.436 \\
23 & 36 & 29.149 \\
23 & 40 & 8.765 \\
23 & 47 & 27.364 \\
23 & 51 & 6.226
\end{tabular} & $\begin{array}{r}\mathrm{s} \\
+0.046 \\
+.079 \\
+.045 \\
+.074 \\
-.024\end{array}$ & $\begin{array}{l}\text { s } \\
64.777 \\
64.589 \\
64.607 \\
64.577 \\
64.516\end{array}$ & $\begin{array}{r}s \\
-0.023 \\
-.081 \\
-.023 \\
+.007 \\
-.034\end{array}$ & 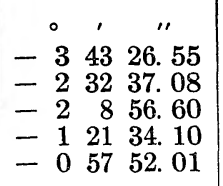 & $\begin{array}{r}\text { '" } \\
+1.25 \\
+.72 \\
+1.00 \\
+.40 \\
+.19\end{array}$ & $\begin{array}{cc}, & \text { " } \\
16 & 6.96 \\
16 & 5.34 \\
16 & 5.60 \\
16 & 5.28 \\
16 & 4.82\end{array}$ & $\begin{aligned} & \prime \prime \\
&-0.53 \\
&=1.37 \\
&=.85 \\
&=.64 \\
&-.84\end{aligned}$ \\
\hline Apr. & $\begin{array}{l}21.0 \\
25.0 \\
11.0 \\
12.0 \\
14.0\end{array}$ & $\begin{array}{l}\mathrm{Ry} \\
\mathrm{Bn} \\
\mathrm{Bn} \\
\mathrm{Bn} \\
\mathrm{P}\end{array}$ & $\underset{W}{E}$ & $\begin{array}{rrr}0 & 2 & 2.050 \\
0 & 16 & 34.780 \\
1 & 18 & 35.617 \\
1 & 22 & 16.238 \\
1 & 29 & 38.328\end{array}$ & $\begin{array}{l}+.030 \\
.020 \\
+.047 \\
+.028 \\
-.022\end{array}$ & $\begin{array}{l}64.436 \\
64.416 \\
64.748 \\
64.797 \\
64.860\end{array}$ & $\begin{array}{l}-.054 \\
-.024 \\
-.032 \\
-.023 \\
-.050\end{array}$ & 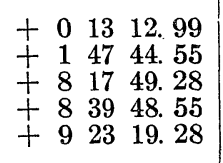 & $\begin{array}{l}-.01 \\
+.55 \\
\overline{+} .22 \\
+.55 \\
+.88\end{array}$ & $\begin{array}{rr}16 & 3.34 \\
16 & 1.92 \\
15 & 58.92 \\
15 & 58.05 \\
15 & 56.02\end{array}$ & $\begin{array}{l}-1.51 \\
-1.84 \\
=.09 \\
-.70 \\
-2.20\end{array}$ \\
\hline & $\begin{array}{l}15.0 \\
19.0 \\
21.0 \\
22.0 \\
25.0\end{array}$ & $\begin{array}{l}\text { Ry } \\
\text { Ry } \\
\text { P } \\
\text { Bn } \\
\text { Bn }\end{array}$ & & $\begin{array}{rrr}1 & 33 & \\
1 & 48 & 9.476 \\
1 & 55 & 36.690 \\
1 & 59 & 20 . \\
2 & 10 & 36.347\end{array}$ & $\begin{array}{r}-.054 \\
.000 \\
+.037 \\
+.023\end{array}$ & $\begin{array}{l}65.206 \\
65.332 \\
65.308 \\
65.463\end{array}$ & $\begin{array}{l}+\quad .016 \\
+\quad .022 \\
-.062 \\
-.117\end{array}$ & $\begin{array}{rrr}+ & 944 & 50.22 \\
+11 & 9 & 13.86 \\
+11 & 50 & \\
+12 & 10 & 38.40 \\
+13 & 10 & 13.29\end{array}$ & $\begin{array}{l}+.52 \\
-.14 \\
+.70 \\
-.21\end{array}$ & $\begin{array}{ll}15 & 56.96 \\
15 & 57.39\end{array}$ & $\begin{array}{l}-1.00 \\
+.47 \\
-2.33 \\
-1.85\end{array}$ \\
\hline May & $\begin{array}{r}28.0 \\
9.0 \\
10.0 \\
17.0 \\
19.0\end{array}$ & $\begin{array}{l}\mathrm{P} \\
\mathrm{Bn} \\
\mathrm{Bn} \\
\mathrm{Ry} \\
\mathrm{P}\end{array}$ & & $\begin{array}{rrr}2 & 21 & 56.189 \\
3 & 4 & 11.283 \\
3 & 8 & 5.118 \\
3 & 35 & 37.631 \\
3 & 43 & 34.837\end{array}$ & $\begin{array}{l}+.001 \\
+.043 \\
+.018 \\
.069 \\
+.017\end{array}$ & $\begin{array}{l}\text { 65. } 854 \\
66.621 \\
66.672 \\
67.380 \\
67.519\end{array}$ & $\begin{array}{l}+.054 \\
+.049 \\
+.078 \\
+.050 \\
+.039\end{array}$ & $\begin{array}{rrr}+14 & 7 & 53.03 \\
+17 & 20 & 23.98 \\
+17 & 36 & 16.20 \\
+19 & 18 & 50.43 \\
+19 & 45 & 15.82\end{array}$ & $\begin{array}{r}-.07 \\
-.12 \\
.00 \\
+.53 \\
+.52\end{array}$ & $\begin{array}{ll}15 & 53.30 \\
15 & 51.76 \\
15 & 50.46 \\
15 & 51.08 \\
15 & 48.61\end{array}$ & $\begin{array}{l}-1.33 \\
-.29 \\
-1.38 \\
+.63 \\
-1.47\end{array}$ \\
\hline June & $\begin{array}{r}20.0 \\
23.0 \\
31.0 \\
2.0 \\
3.0\end{array}$ & $\begin{array}{l}\mathrm{Bn} \\
\mathrm{Bn} \\
\mathrm{Ry} \\
\mathrm{P} \\
\mathrm{Bn}\end{array}$ & & $\begin{array}{rrr}3 & 47 & 34.229 \\
3 & 59 & 35.678 \\
4 & 32 & 1.643 \\
4 & 40 & 12.907 \\
4 & 44 & 19.131\end{array}$ & $\begin{array}{l}+.039 \\
+.0138 \\
+.017 \\
+.067 \\
+.081\end{array}$ & $\begin{array}{l}67.556 \\
67.699 \\
68.339 \\
68.510 \\
68.439\end{array}$ & $\begin{array}{r}-.004 \\
+.081 \\
+.029 \\
+.080 \\
-.041\end{array}$ & $\begin{array}{rrr}+19 & 57 & 58.13 \\
+20 & 34 & 1.96 \\
+21 & 54 & 27.57 \\
+22 & 10 & 50.34 \\
+22 & 18 & 26.31\end{array}$ & $\begin{array}{l}+.33 \\
+.14 \\
+.17 \\
+.74 \\
+.31\end{array}$ & $\begin{array}{ll}15 & 49.84 \\
15 & 47.92 \\
15 & 47.64 \\
15 & 47.48 \\
15 & 46.66\end{array}$ & $\begin{array}{l}-.06 \\
=1.45 \\
=.44 \\
=.32 \\
-1.00\end{array}$ \\
\hline & $\begin{array}{l}10.0 \\
13.0 \\
14.0 \\
16.0 \\
20.0\end{array}$ & $\begin{array}{l}\text { Ry } \\
\text { Ry } \\
\text { Ry } \\
P \\
P\end{array}$ & & $\begin{array}{rrr}5 & 13 & 11.833 \\
5 & 25 & 38.167 \\
5 & 29 & 47.191 \\
5 & 38 & 5.777 \\
5 & 54 & 43.591\end{array}$ & $\begin{array}{r}+.003 \\
+.047 \\
.009 \\
+.057 \\
+.021\end{array}$ & $\begin{array}{l}68.707 \\
68.842 \\
68.866 \\
68.882 \\
68.936\end{array}$ & $\begin{array}{r}-.073 \\
-.018 \\
-.014 \\
-.028 \\
-.004\end{array}$ & $\begin{array}{rrrr}+23 & 0 & 38.96 \\
+23 & 12 & 42.86 \\
+23 & 15 & 55.10 \\
+23 & 21 & \\
+23 & 26 & 29.00\end{array}$ & $\begin{array}{l}+.26 \\
+.56 \\
+.50 \\
-.50\end{array}$ & $\begin{array}{ll}15 & 46.84 \\
15 & 45.46 \\
15 & 44.76 \\
15 & 45.74\end{array}$ & $\begin{array}{l}-.02 \\
-1.13 \\
-1.75 \\
-.36\end{array}$ \\
\hline July & $\begin{array}{l}24.0 \\
27.0 \\
18.0 \\
25.0 \\
27.0\end{array}$ & $\begin{array}{l}\text { Ry } \\
\text { Ry } \\
\text { Ry } \\
\text { Bn } \\
\text { Ry }\end{array}$ & & $\begin{array}{lll}6 & 11 & \\
6 & 23 & 49 . \\
7 & 49 & 51.911 \\
8 & 17 & 46.502 \\
8 & 25 & 39.807\end{array}$ & $\begin{array}{l}+.070 \\
+.111 \\
+.112 \\
+\quad .067\end{array}$ & $\begin{array}{l}\text { 68. } 871 \\
67.771 \\
67.169 \\
67.011\end{array}$ & $\begin{array}{l}+.011 \\
+.001 \\
-.041 \\
-.029\end{array}$ & $\begin{array}{rrr}+23 & 25 & 16.68 \\
+23 & 20 & 2.50 \\
+21 & 2 & 54.51 \\
+19 & 40 & 50.61 \\
+19 & 14 & 24.55\end{array}$ & $\begin{array}{r}-.32 \\
.00 \\
+.91 \\
+.99 \\
+.15\end{array}$ & $\begin{array}{ll}15 & 44.96 \\
15 & 45.56 \\
15 & 46.52 \\
15 & 46.04 \\
15 & 46.23\end{array}$ & $\begin{array}{l}-.93 \\
\overline{0} .21 \\
\pm .33 \\
-.70 \\
-.69\end{array}$ \\
\hline $\begin{array}{l}\text { Aug. } \\
\qquad \begin{array}{r}1 \\
1\end{array}\end{array}$ & $\begin{array}{r}1.0 \\
5.0 \\
8.0 \\
10.0 \\
19.0\end{array}$ & $\begin{array}{l}\text { Ry } \\
\text { P } \\
\text { Bn } \\
\text { Ry } \\
P\end{array}$ & & $\begin{array}{rrr}8 & 45 & 12.958 \\
9 & 0 & 40.899 \\
9 & 12 & 10.269 \\
9 & 20 & \\
9 & 53 & 33.515\end{array}$ & $\begin{array}{l}+.058 \\
+.169 \\
+.039 \\
-.035\end{array}$ & $\begin{array}{l}\text { 66. } 606 \\
66.236 \\
65.956 \\
65.198\end{array}$ & $\begin{array}{l}-.004 \\
-.024 \\
-.044 \\
+.048\end{array}$ & $\begin{array}{rrr}+18 & 2 & 44.88 \\
+17 & 0 & 7.26 \\
+16 & 10 & 13.90 \\
+15 & 35 & 41.68 \\
+12 & 48 & 25.25\end{array}$ & $\begin{array}{r}-1.52 \\
-.24 \\
-.50 \\
+.78 \\
-1.05\end{array}$ & $\begin{array}{ll}15 & 47.56 \\
15 & 46.74 \\
15 & 46.76 \\
15 & 48.34 \\
15 & 49.95\end{array}$ & $\begin{array}{l}+.09 \\
-1.25 \\
-1.68 \\
-.42 \\
-.42\end{array}$ \\
\hline Sept. ${ }^{2}$ & $\begin{array}{r}22.0 \\
24.0 \\
26.0 \\
31.0 \\
2.0\end{array}$ & $\begin{array}{l}\text { Ry } \\
\text { Ry } \\
\text { Ry } \\
\text { Ry } \\
\text { Bn }\end{array}$ & & $\begin{array}{rrr}10 & 4 & 39.883 \\
10 & 12 & 1.945 \\
10 & 19 & 22.232 \\
10 & 37 & 36.880 \\
10 & 44 & 52.587\end{array}$ & $\begin{array}{l}+.003 \\
+.055 \\
.018 \\
+.020 \\
+.117\end{array}$ & $\begin{array}{l}64.906 \\
64.763 \\
64.691 \\
64.409 \\
64.283\end{array}$ & $\begin{array}{r}-.034 \\
-.047 \\
+.011 \\
-.011 \\
-.047\end{array}$ & $\begin{array}{rrr}+11 & 48 & 50.50 \\
+11 & 8 & 10.60 \\
+10 & 26 & 46.68 \\
+8 & 40 & 28.42 \\
+ & 56 & 54.48\end{array}$ & $\begin{array}{l}-.40 \\
+.20 \\
-.52 \\
-.98 \\
-2.72\end{array}$ & $\begin{array}{ll}15 & 51.24 \\
15 & 50.98 \\
15 & 52.80 \\
15 & 52.76 \\
15 & 52.16\end{array}$ & $\begin{array}{l}+.30 \\
\pm .36 \\
+1.06 \\
\pm .06 \\
-1.11\end{array}$ \\
\hline & $\begin{array}{r}7.0 \\
9.0 \\
13.0 \\
15.0 \\
16.0\end{array}$ & $\begin{array}{l}\mathrm{Ry} \\
\mathrm{Bn} \\
\mathrm{Bn} \\
\mathrm{P} \\
\mathrm{Ry}\end{array}$ & & $\begin{array}{rrr}11 & 2 & 56.743 \\
11 & 10 & 9.008 \\
11 & 24 & 31.212 \\
11 & 31 & 41.740 \\
11 & 35 & 16.982\end{array}$ & $\begin{array}{l}+.023 \\
+.178 \\
+.042 \\
+.020 \\
+.072\end{array}$ & $\begin{array}{l}\text { 64. } 181 \\
64.030 \\
63.972 \\
64.010 \\
63.978\end{array}$ & $\begin{array}{l}+.031 \\
-.080 \\
-.068 \\
-.010 \\
-.032\end{array}$ & $\begin{array}{r}+6558.88 \\
+52050.21 \\
+34932.09 \\
+3329.81 \\
+\quad 24022.63\end{array}$ & $\begin{array}{l}+.08 \\
-.99 \\
-2.51 \\
-.89 \\
-.77\end{array}$ & $\begin{array}{ll}15 & 54.72 \\
15 & 53.98 \\
15 & 56.46 \\
15 & 56.35 \\
15 & 56.16\end{array}$ & $\begin{array}{l}+.25 \\
\pm 1.00 \\
+.45 \\
\pm .18 \\
-.63\end{array}$ \\
\hline & $\begin{array}{l}19.0 \\
20.0 \\
22.0 \\
23.0 \\
29.0\end{array}$ & $\begin{array}{l}\text { Ry } \\
\text { Ry } \\
P \\
\mathrm{Bn} \\
\mathrm{P}\end{array}$ & W & $\begin{array}{rrr}11 & 46 & 2.444 \\
11 & 49 & 37.827 \\
11 & 56 & 48.621 \\
12 & 0 & \\
12 & 22 & 1.146\end{array}$ & $\begin{array}{l}+.036 \\
+.087 \\
+.121 \\
+.116\end{array}$ & $\begin{array}{l}64.075 \\
63.952 \\
64.124 \\
64.254\end{array}$ & $\begin{array}{l}+.055 \\
.068 \\
+.074 \\
+.024\end{array}$ & $\begin{array}{r}+13041.69 \\
+12726.12 \\
+02045.46 \\
+0237.23 \\
-22257.31\end{array}$ & $\begin{array}{l}-2.41 \\
-.18 \\
+.06 \\
-.33 \\
-.91\end{array}$ & $\begin{array}{ll}15 & 58.99 \\
15 & 57.02 \\
15 & 59.95 \\
15 & 56.99 \\
15 & 59.42\end{array}$ & $\begin{array}{l}+1.42 \\
+.81 \\
+1.59 \\
-1.63 \\
-.80\end{array}$ \\
\hline
\end{tabular}




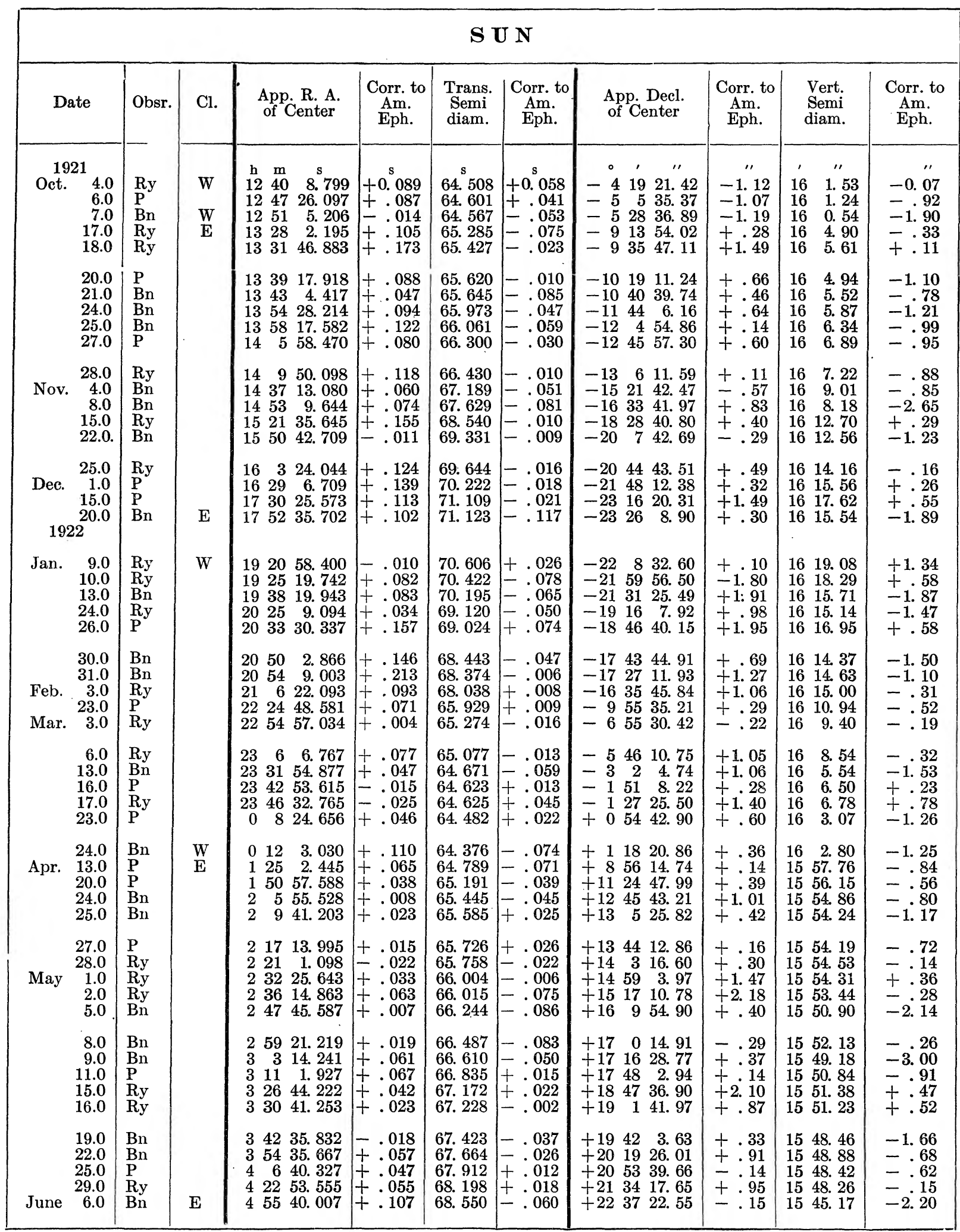




\begin{tabular}{|c|c|c|c|c|c|c|c|c|c|c|c|}
\hline \multicolumn{12}{|c|}{$\mathbf{S} \mathbf{U} \mathbf{N}$} \\
\hline Date & & Obsr. & Cl. & $\begin{array}{l}\text { App. R. A. } \\
\text { of Center }\end{array}$ & $\begin{array}{l}\text { Corr. to } \\
\text { Am. } \\
\text { Eph. }\end{array}$ & $\begin{array}{l}\text { Trans. } \\
\text { Semi } \\
\text { diam. }\end{array}$ & $\begin{array}{c}\text { Corr. to } \\
\text { Am. } \\
\text { Eph. }\end{array}$ & $\begin{array}{l}\text { App. Decl. } \\
\text { of Center }\end{array}$ & $\begin{array}{c}\text { Corr. to } \\
\text { Am. } \\
\text { Eph. }\end{array}$ & $\begin{array}{l}\text { Vert. } \\
\text { Semi } \\
\text { diam. }\end{array}$ & $\begin{array}{c}\text { Corr. to } \\
\text { Am. } \\
\text { Eph. }\end{array}$ \\
\hline $\begin{array}{r}1922 \\
\text { June } \\
1 \\
1 \\
1 \\
2\end{array}$ & $\begin{array}{r}8.0 \\
12.0 \\
15.0 \\
16.0 \\
22.0\end{array}$ & $\begin{array}{l}\mathrm{P} \\
\mathrm{Ry} \\
\mathrm{Bn} \\
\mathrm{Bn} \\
\mathrm{P}\end{array}$ & $\begin{array}{l}\mathrm{E} \\
\mathrm{W}\end{array}$ & \begin{tabular}{rrr}
$\mathrm{h}$ & $\mathrm{m}$ & \multicolumn{1}{c}{$\mathrm{s}$} \\
$\mathbf{5}$ & $\mathbf{3}$ & $\mathbf{5 4} .893$ \\
5 & 20 & 27.984 \\
5 & 32 & 54.951 \\
5 & 37 & 4.351 \\
$\mathbf{6}$ & 2 & 1.970
\end{tabular} & $\begin{array}{r}\mathrm{s} \\
+0.043 \\
+\quad .054 \\
+\quad .001 \\
+.081 \\
+\quad .090\end{array}$ & $\begin{array}{l}\text { s } \\
68.630 \\
68.807 \\
68.804 \\
68.836 \\
68.899\end{array}$ & $\begin{array}{r}\mathrm{s} \\
-0.070 \\
-.023 \\
-.086 \\
-.074 \\
-.041\end{array}$ & \begin{tabular}{rrr}
$\circ$ & \multicolumn{1}{c}{${ }^{\prime \prime}$} \\
+22 & 49 & 14.08 \\
+23 & 8 & 9.01 \\
+23 & 18 & 3.33 \\
+23 & 20 & 31.75 \\
+23 & 26 & 44.99
\end{tabular} & $\begin{array}{c}\prime \prime \\
-0.22 \\
+.81 \\
+.93 \\
+.55 \\
+.69\end{array}$ & \begin{tabular}{ll}
$\prime$ & \multicolumn{1}{c}{} \\
15 & 45.79 \\
15 & 46.33 \\
15 & 45.20 \\
15 & 44.98 \\
15 & 45.41
\end{tabular} & $\begin{aligned} & 1 . \\
&-1.36 \\
&=.40 \\
&=1.25 \\
&=1.39 \\
&-.54\end{aligned}$ \\
\hline July $\begin{array}{r}2 \\
2 \\
3\end{array}$ & $\begin{array}{r}23.0 \\
26.0 \\
30.0 \\
3.0 \\
6.0\end{array}$ & $\begin{array}{l}\text { Ry } \\
\text { Ry } \\
\text { Ry } \\
\text { Bn } \\
P\end{array}$ & & $\begin{array}{rrr}6 & 6 & 11.488 \\
6 & 18 & 40.026 \\
6 & 35 & 15.985 \\
6 & 47 & 40.390 \\
7 & 0 & 1.965\end{array}$ & $\begin{array}{r}-.042 \\
-.014 \\
+.055 \\
+.040 \\
-.025\end{array}$ & $\begin{array}{l}\text { 68. } 931 \\
68.926 \\
68.794 \\
68.646 \\
68.543\end{array}$ & $\begin{array}{r}+.001 \\
+.036 \\
+.014 \\
-.034 \\
-.007\end{array}$ & 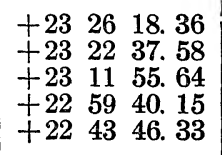 & $\begin{array}{r}-1.34 \\
+.08 \\
.76 \\
+.75 \\
+.23\end{array}$ & $\begin{array}{ll}15 & 46.55 \\
15 & 46.24 \\
15 & 45.85 \\
15 & 44.06 \\
15 & 45.28\end{array}$ & $\begin{array}{l}+.65 \\
+.46 \\
+.15 \\
+1.63 \\
-.43\end{array}$ \\
\hline & $\begin{array}{r}7.0 \\
13.0 \\
17.0 \\
18.0 \\
21.0\end{array}$ & $\begin{array}{l}\text { Ry } \\
\text { Ry } \\
P \\
\text { Bn } \\
\text { Ry }\end{array}$ & & $\begin{array}{rrr}7 & 4 & 8.500 \\
7 & 28 & 39.358 \\
7 & 45 & \\
7 & 48 & 52.537 \\
8 & 0 & 54.371\end{array}$ & $\begin{array}{r}+.010 \\
+.0108 \\
+.047 \\
+.011\end{array}$ & $\begin{array}{l}68.449 \\
68.122 \\
\\
67.686 \\
67.571\end{array}$ & $\begin{array}{r}-.051 \\
-.028 \\
-.104 \\
+.021\end{array}$ & $\begin{array}{rrr}+22 & 37 & 40.97 \\
+21 & 52 & 59.92 \\
+21 & 15 & 40.93 \\
+21 & 5 & \\
+20 & 32 & 33.30\end{array}$ & $\begin{array}{l}+.27 \\
+.18 \\
-.33 \\
-.90\end{array}$ & $\begin{array}{ll}15 & 45.28 \\
15 & 44.73 \\
15 & 44.94 \\
& \\
15 & 46.44\end{array}$ & $\begin{array}{l}-.45 \\
-1.17 \\
-1.13 \\
+.11\end{array}$ \\
\hline Aug. & $\begin{array}{r}24.0 \\
28.0 \\
31.0 \\
3.0 \\
4.0\end{array}$ & $\begin{array}{l}\mathrm{P} \\
\mathrm{Ry} \\
\mathrm{P} \\
\mathrm{P} \\
\mathrm{Ry}\end{array}$ & W & $\begin{array}{rrr}8 & 12 & 51.368 \\
8 & 28 & 38.971 \\
8 & 40 & 23.298 \\
\cdot 8 & 52 & 1.852 \\
8 & 55 & 53.503\end{array}$ & $\begin{array}{r}+.098 \\
+.041 \\
+.0108 \\
+.042 \\
+.063\end{array}$ & $\begin{array}{l}\text { 67. } 293 \\
66.956 \\
66.754 \\
66.495 \\
66.294\end{array}$ & $\begin{array}{l}-.017 \\
-.024 \\
+.034 \\
+.035 \\
-.076\end{array}$ & $\begin{array}{rrr}+19 & 56 & 34.91 \\
+19 & 3 & 59.13 \\
+18 & 21 & 15.59 \\
+17 & 35 & 48.94 \\
+17 & 20 & 4.46\end{array}$ & $\begin{array}{l}-1.09 \\
-1.47 \\
=.01 \\
+.44 \\
-.44\end{array}$ & $\begin{array}{ll}15 & 45.38 \\
15 & 46.68 \\
15 & 46.81 \\
15 & 47.86 \\
15 & 48.17\end{array}$ & $\begin{array}{l}-1.19 \\
-\quad .29 \\
-.52 \\
+.14 \\
-.31\end{array}$ \\
\hline & $\begin{array}{l}18.0 \\
21.0 \\
22.0 \\
24.0 \\
28.0\end{array}$ & $\begin{array}{l}\mathrm{Ry} \\
\mathrm{P} \\
\mathrm{P} \\
\mathrm{P} \\
\mathrm{P}\end{array}$ & $\mathrm{E}$ & $\begin{array}{rrr}9 & 48 & 55.629 \\
10 & 0 & 4.167 \\
10 & 3 & 46.054 \\
10 & 11 & 8.494 \\
10 & 25 & 48.339\end{array}$ & $\begin{array}{l}+.099 \\
+.097 \\
+.064 \\
+.024 \\
+.079\end{array}$ & $\begin{array}{l}\text { 65. } 217 \\
65.001 \\
64.897 \\
64.773 \\
64.575\end{array}$ & $\begin{array}{l}-.013 \\
-.019 \\
-.053 \\
-.047 \\
-.005\end{array}$ & 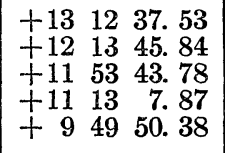 & $\begin{array}{l} \pm .17 \\
\pm .64 \\
. .12 \\
+.07 \\
-.52\end{array}$ & $\begin{array}{ll}15 & 49.26 \\
15 & 51.15 \\
15 & 50.54 \\
15 & 50.10 \\
15 & 51.18\end{array}$ & $\begin{array}{l}-.81 \\
+.52 \\
-.29 \\
-1.13 \\
-.92\end{array}$ \\
\hline Sept. & $\begin{array}{r}29.0 \\
31.0 \\
5.0 \\
7.0 \\
11.0\end{array}$ & $\begin{array}{l}\mathrm{Bn} \\
\mathrm{Bn} \\
\mathrm{Bn} \\
\mathrm{Bn} \\
\mathrm{Ry}\end{array}$ & & $\begin{array}{rrr}10 & 29 & 27.311 \\
10 & 36 & 44.127 \\
10 & 54 & 50.481 \\
11 & 2 & 3.308 \\
11 & 16 & 26.733\end{array}$ & $\begin{array}{l}+.111 \\
+.0127 \\
+.051 \\
+.088 \\
+.093\end{array}$ & $\begin{array}{l}64.444 \\
64.420 \\
64.148 \\
64.096 \\
63.989\end{array}$ & $\begin{array}{l}-.086 \\
-.010 \\
-.082 \\
-.074 \\
-.081\end{array}$ & 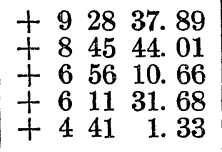 & $\begin{array}{l}+.29 \\
.39 \\
+.06 \\
+.28 \\
+.03\end{array}$ & $\begin{array}{ll}15 & 52.11 \\
15 & 51.73 \\
15 & 52.87 \\
15 & 53.25 \\
15 & 54.64\end{array}$ & $\begin{array}{l}-.21 \\
-1.05 \\
-1.09 \\
-1.19 \\
-.77\end{array}$ \\
\hline & $\begin{array}{l}12.0 \\
14.0 \\
15.0 \\
18.0 \\
25.0\end{array}$ & $\begin{array}{l}\mathrm{P} \\
\mathrm{P} \\
\mathrm{Ry} \\
\mathrm{Ry} \\
\mathrm{Ry}\end{array}$ & & $\begin{array}{rrr}11 & 20 & \\
11 & 27 & 13.058 \\
11 & 30 & 48.452 \\
11 & 41 & 34.442 \\
12 & 6 & 43.295\end{array}$ & $\begin{array}{l}+.048 \\
+.102 \\
+\quad .132 \\
+\quad .035\end{array}$ & $\begin{array}{l}64.045 \\
63.966 \\
64.009 \\
64.143\end{array}$ & $\begin{array}{l}+.015 \\
-.054 \\
-.001 \\
+.043\end{array}$ & $\begin{array}{r}+41810.72 \\
+33216.49 \\
+39 \\
+13.78 \\
+15942.63 \\
-04340.97\end{array}$ & $\begin{array}{l}+.02 \\
+\quad .29 \\
+.98 \\
+\quad .33 \\
+1.33\end{array}$ & $\begin{array}{ll}15 & 54.46 \\
15 & 55.76 \\
15 & 55.88 \\
15 & 57.74 \\
15 & 59.52\end{array}$ & $\begin{array}{r}-1.19 \\
-.39 \\
-.52 \\
+.57 \\
+.47\end{array}$ \\
\hline Oct. & $\begin{array}{r}26.0 \\
28.0 \\
29.0 \\
3.0 \\
5.0\end{array}$ & $\begin{array}{l}\mathrm{P} \\
\mathrm{P} \\
\mathrm{Ry} \\
\mathrm{Ry} \\
\mathrm{P}\end{array}$ & & $\begin{array}{rrr}12 & 10 & 19.407 \\
12 & 17 & 31.862 \\
12 & 21 & 8.406 \\
12 & 35 & 37.084 \\
12 & 42 & 53.212\end{array}$ & $\begin{array}{l}+.157 \\
+.112 \\
+.116 \\
+.134 \\
+.122\end{array}$ & $\begin{array}{l}\text { 64. } 125 \\
\text { 64. } 247 \\
64.152 \\
64.369 \\
64.494\end{array}$ & $\begin{array}{l}-.005 \\
+.057 \\
-.068 \\
.021 \\
+.004\end{array}$ & 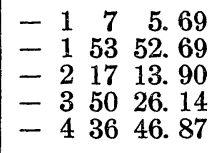 & $\begin{array}{l}+.41 \\
\pm .19 \\
+.50 \\
-.74 \\
-.67\end{array}$ & $\begin{array}{rr}15 & 59.28 \\
15 & 59.24 \\
15 & 59.72 \\
16 & 1.07 \\
16 & 0.20\end{array}$ & $\begin{array}{l}=.05 \\
=.65 \\
=.45 \\
=.22 \\
-1.65\end{array}$ \\
\hline & $\begin{array}{l}12.0 \\
13.0 \\
16.0 \\
19.0 \\
20.0\end{array}$ & $\begin{array}{l}\mathrm{P} \\
\text { Ry } \\
\text { Ry } \\
\mathrm{P} \\
\mathrm{Bn}\end{array}$ & & $\begin{array}{rrr}13 & 8 & 32.316 \\
13 & 12 & 14.005 \\
13 & 23 & 22.696 \\
13 & 34 & 36.682 \\
13 & 38 & 22.481\end{array}$ & $\begin{array}{r}+.106 \\
+.025 \\
+.096 \\
+.162 \\
+.071\end{array}$ & $\begin{array}{l}\text { 64. } 960 \\
64.998 \\
65.330 \\
\text { 65. } 594 \\
65.564\end{array}$ & $\begin{array}{l}+.020 \\
-.012 \\
+.080 \\
+.084 \\
-.036\end{array}$ & $\begin{array}{rrr}7 & 16 & 54.75 \\
-7 & 39 & 26.31 \\
-8 & 46 & 22.25 \\
-9 & 52 & 12.20 \\
-10 & 13 & 53.18\end{array}$ & $\begin{array}{l}-.65 \\
-.21 \\
+.75 \\
+1.30 \\
+.42\end{array}$ & $\begin{array}{ll}16 & 3.69 \\
16 & 5.62 \\
16 & 3.05 \\
16 & 5.63 \\
16 & 4.08\end{array}$ & $\begin{array}{l}-.07 \\
+1.59 \\
+1.79 \\
-.01 \\
-1.83\end{array}$ \\
\hline Nov. & $\begin{array}{r}27.0 \\
30.0 \\
31.0 \\
9.0 \\
10.0\end{array}$ & $\begin{array}{l}\text { Ry } \\
R y \\
R y \\
P \\
\text { Ry }\end{array}$ & & $\begin{array}{rrr}14 & 5 & 2.184 \\
14 & 16 & 38.426 \\
14 & 20 & 31.973 \\
14 & 56 & 10.521 \\
15 & 0 & 12.205\end{array}$ & $\begin{array}{r}+.044 \\
+.086 \\
+.063 \\
+.141 \\
+.005\end{array}$ & $\begin{array}{l}\text { 66. } 291 \\
66.687 \\
66.768 \\
67.789 \\
67.986\end{array}$ & $\begin{array}{r}+.019 \\
+.057 \\
+.018 \\
+.011 \\
+.066\end{array}$ & $\begin{array}{rrr}-12 & 40 & 58.62 \\
-13 & 41 & 12.69 \\
-14 & 0 & 49.88 \\
-16 & 46 & 43.34 \\
-17 & 3 & 49.38\end{array}$ & $\begin{array}{l}+.78 \\
.79 \\
+.52 \\
+.66 \\
+.72\end{array}$ & $\begin{array}{rr}16 & 7.70 \\
16 & 8.70 \\
16 & 10.52 \\
16 & 11.14 \\
16 & 11.98\end{array}$ & $\begin{array}{l}-.10 \\
+.11 \\
+1.68 \\
+.12 \\
+.74\end{array}$ \\
\hline & $\begin{array}{l}13.0 \\
14.0 \\
16.0 \\
17.0 \\
20.0\end{array}$ & $\begin{array}{l}\text { Ry } \\
R y \\
\text { P } \\
B n \\
B n\end{array}$ & $\mathbf{E}$ & $\begin{array}{lll}15 & 12 & 23.025 \\
15 & 16 & 28.208 \\
15 & 24 & 41.561 \\
15 & 28 & 49.270 \\
15 & 41 & 18.158\end{array}$ & $\begin{array}{r}+.135 \\
+.018 \\
+.141 \\
.040 \\
+.128\end{array}$ & $\begin{array}{l}\text { 68. } 219 \\
68.340 \\
68.676 \\
68.710 \\
69.022\end{array}$ & $\begin{array}{l}-.061 \\
\overline{.} .060 \\
+.046 \\
-.040 \\
-.068\end{array}$ & $\begin{array}{rrr}-17 & 53 & 22.82 \\
-18 & 9 & 15.93 \\
-18 & 40 & 8.56 \\
-18 & 55 & 2.17 \\
-19 & 37 & 48.13\end{array}$ & $\begin{array}{l}-.42 \\
+.57 \\
-1.46 \\
+.83 \\
-.33\end{array}$ & $\begin{array}{ll}16 & 12.20 \\
16 & 12.01 \\
16 & 11.51 \\
16 & 12.62 \\
16 & 13.24\end{array}$ & $\begin{array}{l} \pm .31 \\
\pm .09 \\
-1.01 \\
=.10 \\
-.09\end{array}$ \\
\hline
\end{tabular}

$137506^{\circ}-33-8$ 


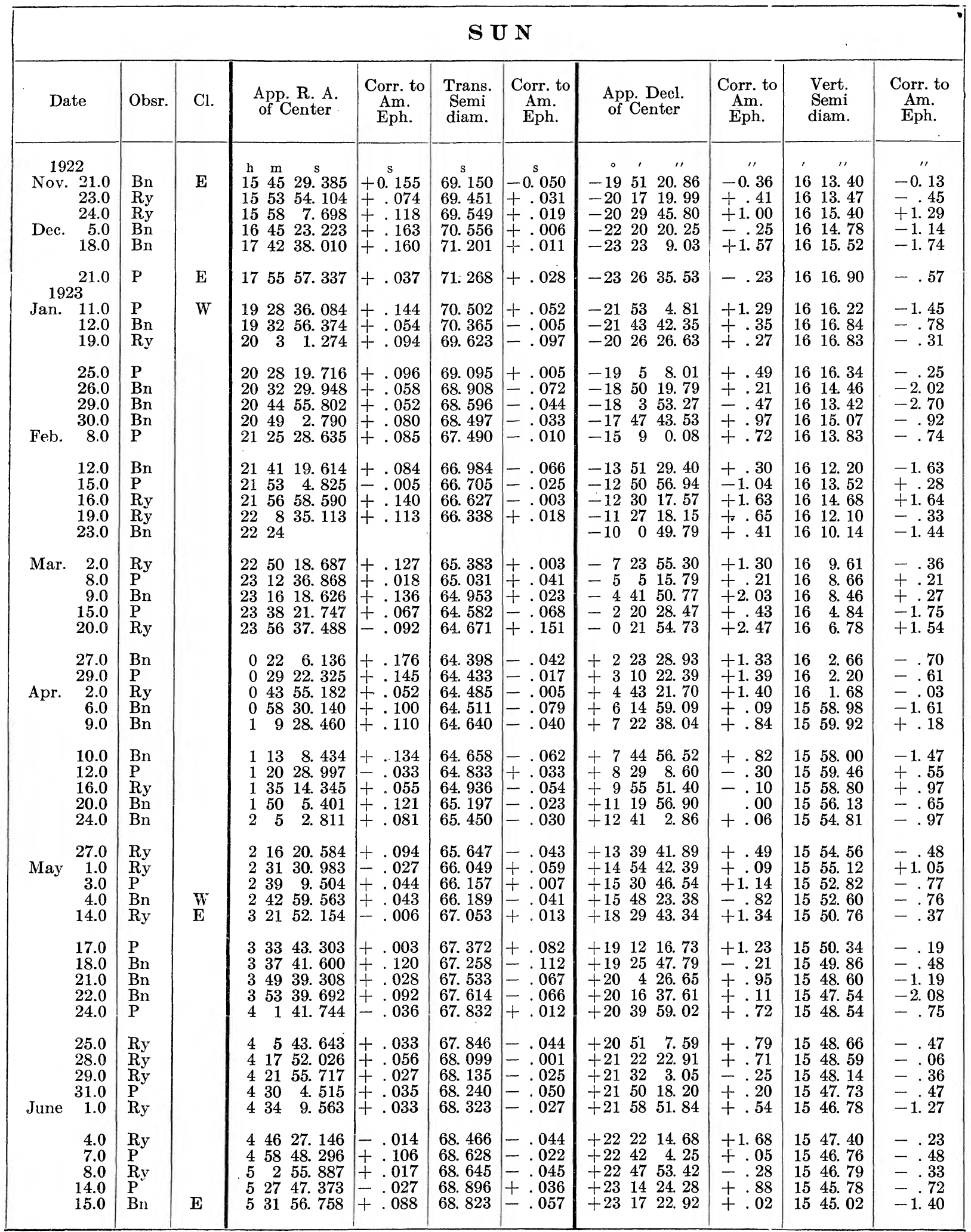




\begin{tabular}{|c|c|c|c|c|c|c|c|c|c|c|c|}
\hline \multicolumn{12}{|c|}{$\mathbf{S} \mathbf{U} \mathbf{N}$} \\
\hline Dat & & Obsr. & Cl. & $\begin{array}{l}\text { App. R. A. } \\
\text { of Center }\end{array}$ & $\begin{array}{c}\text { Corr. to } \\
\text { Am. } \\
\text { Eph. }\end{array}$ & $\begin{array}{l}\text { Trans. } \\
\text { Semi } \\
\text { diam. }\end{array}$ & $\begin{array}{c}\text { Corr. to } \\
\text { Am. } \\
\text { Eph. }\end{array}$ & $\begin{array}{l}\text { App. Decl. } \\
\text { of Center }\end{array}$ & $\begin{array}{c}\text { Corr. to } \\
\text { Am. } \\
\text { Eph. }\end{array}$ & $\begin{array}{l}\text { Vert. } \\
\text { Semi } \\
\text { diam. }\end{array}$ & $\begin{array}{c}\text { Corr. to } \\
\text { Am. } \\
\text { Eph. }\end{array}$ \\
\hline $\begin{array}{l}\text { 192: } \\
\text { June }\end{array}$ & $\begin{array}{l}3 \\
18.0 \\
19.0 \\
21.0 \\
22.0 \\
25.0\end{array}$ & $\begin{array}{l}\mathrm{Bn} \\
\mathrm{Bn} \\
\mathrm{Ry} \\
\mathrm{Ry} \\
\mathrm{Ry}\end{array}$ & $\mathrm{E}$ & \begin{tabular}{rrr}
$\mathrm{h}$ & $\mathrm{m}$ & \multicolumn{1}{c}{$\mathrm{s}$} \\
5 & 44 & 25.088 \\
5 & 48 & 34.821 \\
5 & 56 & 53.932 \\
6 & 1 & 3.638 \\
6 & 13 & 31.976
\end{tabular} & $\begin{array}{r}\mathrm{s} \\
-0.052 \\
+\quad .071 \\
+.058 \\
+.068 \\
+.066\end{array}$ & $\begin{array}{l}\text { s } \\
68.868 \\
68.834 \\
68.883 \\
68.922 \\
68.845\end{array}$ & $\begin{array}{r}\mathrm{s} \\
-0.062 \\
-.096 \\
-.057 \\
-.018 \\
-.055\end{array}$ & $\begin{array}{rcc}\circ & \prime & \prime \prime \\
+23 & 23 & 54.34 \\
+23 & 25 & 14.30 \\
+23 & 26 & 40.92 \\
+23 & 26 & 47.56 \\
+23 & 24 & 37.25\end{array}$ & $\begin{array}{r}1 . \\
+1.04 \\
+.40 \\
+.22 \\
+.56 \\
+.45\end{array}$ & $\begin{array}{lc}\prime & \prime \prime \\
15 & 45.10 \\
15 & 45.84 \\
15 & 46.15 \\
15 & 46.92 \\
15 & 44.54\end{array}$ & $\begin{array}{r} \\
-1.10 \\
-.30 \\
+.12 \\
+.94 \\
-1.31\end{array}$ \\
\hline July & $\begin{array}{r}29.0 \\
3.0 \\
5.0 \\
6.0 \\
10.0\end{array}$ & $\begin{array}{l}\mathrm{Bn} \\
\mathrm{P} \\
\mathrm{P} \\
\mathrm{Ry} \\
\mathrm{P}\end{array}$ & & $\begin{array}{llr}6 & 30 & \\
6 & 46 & 40.872 \\
6 & 54 & 55.851 \\
6 & 59 & 2.836 \\
7 & 15 & 27 . \\
295\end{array}$ & $\begin{array}{l}+.032 \\
+.071 \\
+.046 \\
-.095\end{array}$ & $\begin{array}{l}68.669 \\
68.617 \\
68.492 \\
68.310\end{array}$ & $\begin{array}{l}-.021 \\
+.017 \\
-.068 \\
-.030\end{array}$ & $\begin{array}{rrr}+23 & 15 & 57.12 \\
+23 & 0 & 46.08 \\
+22 & 50 & 45.42 \\
+22 & 45 & 9.22 \\
+22 & 18 & 48.53\end{array}$ & $\begin{array}{r}-.18 \\
+.08 \\
+.12 \\
+.12 \\
-.07\end{array}$ & $\begin{array}{ll}15 & 44.38 \\
15 & 44.43 \\
15 & 45.52 \\
15 & 45.42 \\
15 & 44.28\end{array}$ & $\begin{array}{l}-1.36 \\
=1.24 \\
=.14 \\
=.24 \\
-1.43\end{array}$ \\
\hline & $\begin{array}{l}17.0 \\
19.0 \\
20.0 \\
23.0 \\
26.0\end{array}$ & $\begin{array}{l}\mathrm{Bn} \\
\mathrm{Bn} \\
\mathrm{Ry} \\
\mathrm{Ry} \\
\mathrm{Bn}\end{array}$ & & $\begin{array}{rrr}7 & 43 & 54.079 \\
7 & 51 & 57.148 \\
7 & 55 & 57.925 \\
8 & 7 & 56.515 \\
8 & 19 & 49.755\end{array}$ & $\begin{array}{r}+.029 \\
+.028 \\
+.115 \\
+.095 \\
+.065\end{array}$ & $\begin{array}{l}67.799 \\
67.684 \\
67.598 \\
67.401 \\
67.105\end{array}$ & $\begin{array}{l}-.081 \\
-.046 \\
-.052 \\
-.009 \\
-.065\end{array}$ & $\begin{array}{rrr}+21 & 18 & 1.43 \\
+20 & 57 & 22.36 \\
+20 & 46 & 29.36 \\
+20 & 11 & 49.43 \\
+19 & 34 & 6.15\end{array}$ & $\begin{array}{r}\overline{0} .07 \\
+.96 \\
.04 \\
+.93 \\
+.64\end{array}$ & $\begin{array}{ll}15 & 44.88 \\
15 & 45.00 \\
15 & 46.47 \\
15 & 46.76 \\
15 & 45.46\end{array}$ & $\begin{array}{l}-1.12 \\
-1.14 \\
+.25 \\
+.29 \\
-1.29\end{array}$ \\
\hline Aug. & $\begin{array}{r}27.0 \\
6.0 \\
7.0 \\
13.0 \\
14.0\end{array}$ & $\begin{array}{l}\mathrm{Ry} \\
\mathrm{Bn} \\
\mathrm{P} \\
\mathrm{Bn} \\
\mathrm{P}\end{array}$ & & $\begin{array}{rrr}8 & 23 & 46.342 \\
9 & 2 & 39.209 \\
9 & 6 & 29.231 \\
9 & 29 & 17.684 \\
9 & 33 & 3.609\end{array}$ & $\begin{array}{r}+.092 \\
+.079 \\
+.051 \\
+.174 \\
+.029\end{array}$ & $\begin{array}{l}\text { 67. } 031 \\
66.167 \\
66.155 \\
65.643 \\
65.500\end{array}$ & $\begin{array}{r}-.049 \\
.043 \\
+.025 \\
+.013 \\
-.050\end{array}$ & 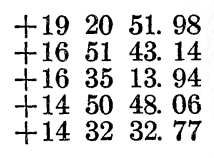 & $\begin{array}{l}+.08 \\
\pm .86 \\
=.26 \\
-.24 \\
+.17\end{array}$ & $\begin{array}{ll}15 & 46.30 \\
15 & 46.98 \\
15 & 47.43 \\
15 & 48.24 \\
15 & 48.76\end{array}$ & $\begin{array}{l}-.55 \\
=1.04 \\
=.73 \\
=.85 \\
-.50\end{array}$ \\
\hline & $\begin{array}{l}16.0 \\
20.0 \\
21.0 \\
30.0 \\
31.0\end{array}$ & $\begin{array}{l}\mathrm{P} \\
\mathrm{Bn} \\
\mathrm{P} \\
\mathrm{Bn} \\
\mathrm{Ry}\end{array}$ & & $\begin{array}{rrr}9 & 40 & 34.122 \\
9 & 55 & 28.676 \\
9 & 59 & 10.935 \\
10 & 32 & 12.101 \\
10 & 35 & 50.367\end{array}$ & $\begin{array}{l}+.062 \\
+.126 \\
+.045 \\
+.041 \\
+.067\end{array}$ & $\begin{array}{l}65.398 \\
65.084 \\
65.044 \\
64.443 \\
64.443\end{array}$ & $\begin{array}{r}+.008 \\
.016 \\
+.014 \\
.047 \\
+.003\end{array}$ & $\begin{array}{rrr}+13 & 55 & 19.25 \\
+12 & 38 & 17.74 \\
+12 & 18 & 32.00 \\
+\quad 912 & 30.20 \\
+ & 51 & 1.32\end{array}$ & $\begin{array}{r}-.35 \\
+.04 \\
+.40 \\
+.10 \\
+.08\end{array}$ & $\begin{array}{ll}15 & 50.44 \\
15 & 50.18 \\
15 & 49.76 \\
15 & 51.02 \\
15 & 52.24\end{array}$ & $\begin{array}{l} \pm .83 \\
\pm .19 \\
=.81 \\
=1.44 \\
-.44\end{array}$ \\
\hline $\begin{array}{r}\text { Sept. } \\
\text {. }\end{array}$ & $\begin{array}{r}4.0 \\
10.0 \\
13.0 \\
14.0 \\
17.0\end{array}$ & $\begin{array}{l}\mathrm{Bn} \\
\mathrm{Ry} \\
\mathrm{Bn} \\
\mathrm{Ry} \\
\mathrm{Ry}\end{array}$ & & $\begin{array}{rrrr}10 & 50 & 20 . & 404 \\
11 & 11 & 58.742 \\
11 & 22 & 45.796 \\
11 & 26 & 21.181 \\
11 & 37 & 7.090\end{array}$ & $\begin{array}{r}+.024 \\
+.062 \\
+.126 \\
+.071 \\
+.080\end{array}$ & $\begin{array}{l}64.195 \\
64.101 \\
64.002 \\
64.067 \\
64.031\end{array}$ & $\begin{array}{r}+.065 \\
+.011 \\
.038 \\
+.047 \\
+.021\end{array}$ & $\begin{array}{r}+72343.61 \\
+59 \\
+517.32 \\
+4048.36 \\
+33749.77 \\
+22832.54\end{array}$ & $\begin{array}{l}-.89 \\
\overline{0} .18 \\
+.06 \\
+.07 \\
+.44\end{array}$ & $\begin{array}{ll}15 & 51.45 \\
15 & 55.46 \\
15 & 55.01 \\
15 & 56.83 \\
15 & 57.38\end{array}$ & $\begin{array}{l}-2.13 \\
+.46 \\
.76 \\
+.80 \\
+.55\end{array}$ \\
\hline Oct. & $\begin{array}{r}24.0 \\
28.0 \\
1.0 \\
2.0 \\
4.0\end{array}$ & $\begin{array}{l}\text { Ry } \\
\text { Ry } \\
\text { Ry } \\
\text { P } \\
\text { P }\end{array}$ & & $\begin{array}{rrr}12 & 2 & 14.303 \\
12 & 16 & 37.969 \\
12 & 27 & 27.997 \\
12 & 31 & 5.250 \\
12 & 38 & 20.705\end{array}$ & $\begin{array}{r}+.093 \\
+.089 \\
+.047 \\
+.060 \\
+.045\end{array}$ & $\begin{array}{l}\text { 64. } 075 \\
64.160 \\
64.206 \\
64.327 \\
64.370\end{array}$ & $\begin{array}{l}+.005 \\
-.020 \\
-.084 \\
-.003 \\
-.050\end{array}$ & 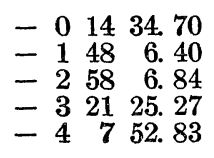 & $\begin{array}{l}-1.10 \\
=1.40 \\
=.14 \\
-1.07 \\
-.43\end{array}$ & $\begin{array}{rr}15 & 58.78 \\
16 & 0.43 \\
15 & 58.90 \\
16 & 1.79 \\
16 & 0.62\end{array}$ & $\begin{array}{l}+.06 \\
+.62 \\
+1.72 \\
+.90 \\
-.81\end{array}$ \\
\hline & $\begin{array}{r}5.0 \\
8.0 \\
9.0 \\
11.0 \\
12.0\end{array}$ & $\begin{array}{l}\text { Ry } \\
\text { Ry } \\
P \\
P \\
\text { Ry }\end{array}$ & & $\begin{array}{rrr}12 & 41 & 58.979 \\
12 & 52 & 56.145 \\
12 & 56 & 35.889 \\
13 & 3 & 57.083 \\
13 & 7 & 38.264\end{array}$ & $\begin{array}{r}+.059 \\
+.135 \\
+.019 \\
+.133 \\
+.084\end{array}$ & $\begin{array}{l}64.505 \\
64.634 \\
64.735 \\
64.866 \\
64.833\end{array}$ & $\begin{array}{r}+.035 \\
.006 \\
+.025 \\
+.026 \\
-.077\end{array}$ & 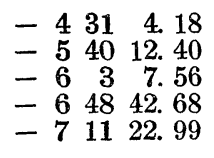 & $\begin{array}{l}-1.68 \\
=.30 \\
-.36 \\
+.12 \\
-.49\end{array}$ & $\begin{array}{ll}16 & 1.99 \\
16 & 3.26 \\
16 & 2.59 \\
16 & 3.64 \\
16 & 2.48\end{array}$ & $\begin{array}{l}+.29 \\
+.74 \\
+.21 \\
+.28 \\
-1.16\end{array}$ \\
\hline Nov. & $\begin{array}{r}18.0 \\
22.0 \\
1.0 \\
2.0 \\
9.0\end{array}$ & $\begin{array}{l}\mathrm{P} \\
\mathrm{Ry} \\
\mathrm{P} \\
\mathrm{Ry} \\
\mathrm{Bn}\end{array}$ & $\underset{W}{\mathrm{E}}$ & $\begin{array}{lll}13 & 29 & 56.315 \\
13 & 44 & 59.790 \\
14 & 23 & 27.473 \\
14 & 27 & 22.523 \\
14 & 55 & 11.096\end{array}$ & $\begin{array}{l}+.035 \\
-.050 \\
+.003 \\
+.033 \\
+.006\end{array}$ & $\begin{array}{l}65.459 \\
65.765 \\
66.843 \\
66.908 \\
67.802\end{array}$ & $\begin{array}{l}+.059 \\
.005 \\
+.013 \\
.042 \\
+.032\end{array}$ & $\begin{array}{rrr}-9 & 25 & 3.65 \\
-10 & 51 & 31.41 \\
-14 & 15 & 26.10 \\
-14 & 34 & 39.57 \\
-16 & 42 & 28.73\end{array}$ & $\begin{array}{l}=.15 \\
=.01 \\
=.60 \\
\pm .83 \\
-.13\end{array}$ & $\begin{array}{rr}16 & 5.15 \\
16 & 6.28 \\
16 & 8.98 \\
16 & 8.00 \\
16 & 10.43\end{array}$ & $\begin{array}{l}=.17 \\
=.14 \\
=.02 \\
=1.25 \\
-.49\end{array}$ \\
\hline & $\begin{array}{l}13.0 \\
19.0 \\
20.0 \\
22.0 \\
27.0\end{array}$ & $\begin{array}{l}\mathrm{P} \\
\mathrm{Ry} \\
\mathrm{Ry} \\
\mathrm{P} \\
\mathrm{Bn}\end{array}$ & & $\begin{array}{rrr}15 & 11 & 23.216 \\
15 & 36 & 6.080 \\
15 & 40 & 16.150 \\
15 & 49 & \\
16 & 9 & 48.655\end{array}$ & $\begin{array}{l}+.136 \\
+.070 \\
+.120 \\
+.105\end{array}$ & $\begin{array}{l}68.243 \\
68.946 \\
69.087 \\
69.817\end{array}$ & $\begin{array}{r}-.007 \\
-.004 \\
+.017 \\
-.003\end{array}$ & $\begin{array}{rrr}-17 & 49 & 26.27 \\
-19 & 20 & 26.02 \\
-19 & 34 & 24.90 \\
-20 & 1 & 18.76 \\
-21 & 2 & 2.24\end{array}$ & $\begin{array}{l}+.03 \\
+.08 \\
+.10 \\
-.26 \\
-1.04\end{array}$ & $\begin{array}{ll}16 & 10.80 \\
16 & 14.01 \\
16 & 12.54 \\
16 & 12.58 \\
16 & 12.04\end{array}$ & $\begin{array}{l}-1.04 \\
+.87 \\
-.81 \\
-1.16 \\
-2.61\end{array}$ \\
\hline Dec. & $\begin{array}{r}3.0 \\
4.0 \\
7.0 \\
11.0 \\
14.0\end{array}$ & $\begin{array}{l}\mathrm{P} \\
\mathrm{Bn} \\
\mathrm{P} \\
\mathrm{Bn} \\
\mathrm{P}\end{array}$ & W & $\begin{array}{rrr}16 & 35 & 37.044 \\
16 & 39 & 57.519 \\
16 & 53 & 2.235 \\
17 & 10 & 35.666 \\
17 & 23 & 49.859\end{array}$ & $\begin{array}{l}+.084 \\
+.139 \\
+.085 \\
+.076 \\
+.049\end{array}$ & $\begin{array}{l}\text { 70. } 329 \\
70.361 \\
70.708 \\
70.915 \\
71.098\end{array}$ & $\begin{array}{r}-.041 \\
-.089 \\
+.038 \\
.005 \\
+.028\end{array}$ & $\begin{array}{rrr}-22 & 1 & 49.66 \\
-22 & 10 & 19.86 \\
-22 & 33 & 14.68 \\
-22 & 57 & 39.31 \\
-23 & 11 & 9.85\end{array}$ & $\begin{array}{l}-1.36 \\
-1.06 \\
+.22 \\
+.81 \\
+.65\end{array}$ & $\begin{array}{ll}16 & 14.66 \\
16 & 13.96 \\
16 & 15.10 \\
16 & 16.04 \\
16 & 16.94\end{array}$ & $\begin{array}{r}-.92 \\
-1.76 \\
-1.02 \\
-.57 \\
.00\end{array}$ \\
\hline
\end{tabular}




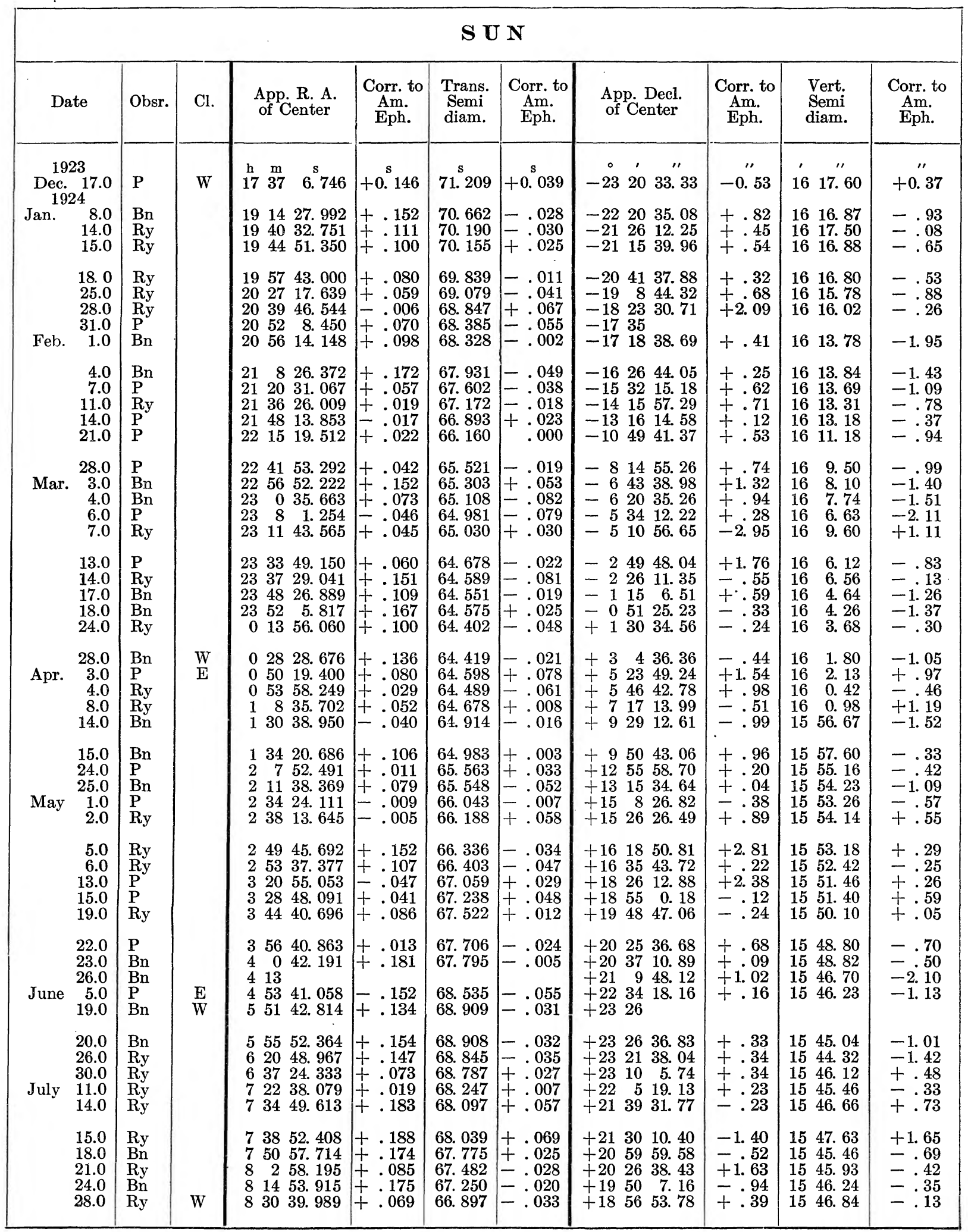




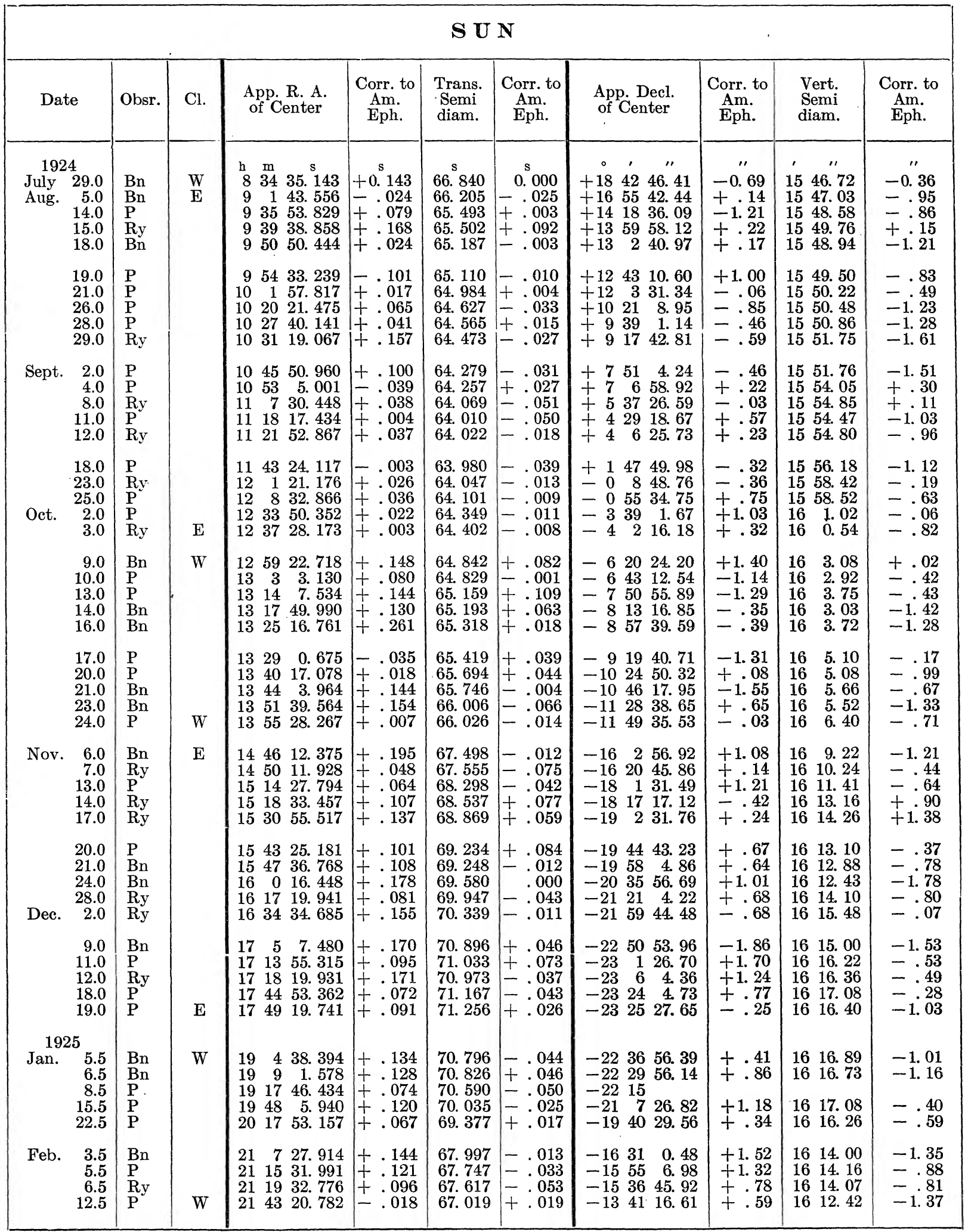




\begin{tabular}{|c|c|c|c|c|c|c|c|c|c|c|c|c|c|}
\hline \multicolumn{14}{|c|}{ MOON } \\
\hline \multirow{2}{*}{\multicolumn{2}{|c|}{ Date }} & \multirow[b]{2}{*}{ Obsr. } & \multirow[b]{2}{*}{ Cl. } & \multirow[b]{2}{*}{$\operatorname{Limb}$} & \multirow{2}{*}{$\begin{array}{l}\text { App. R. A. } \\
\text { of Center }\end{array}$} & \multicolumn{3}{|c|}{ Corr. to- } & \multirow[b]{2}{*}{ Limb } & \multirow{2}{*}{$\begin{array}{l}\text { App. Decl. } \\
\text { of Center }\end{array}$} & \multicolumn{3}{|c|}{ Corr. to- } \\
\hline & & & & & & $\begin{array}{l}\text { Am. } \\
\text { Eph. }\end{array}$ & $\begin{array}{l}\text { Tab. } \\
\text { R. A. }\end{array}$ & $\begin{array}{l}\text { Tab. } \\
\text { Long. }\end{array}$ & & & $\begin{array}{l}\text { Am. } \\
\text { Eph. }\end{array}$ & $\begin{array}{l}\text { Tab. } \\
\text { Decl. }\end{array}$ & $\begin{array}{l}\text { Tab. } \\
\text { Lat. }\end{array}$ \\
\hline $\begin{array}{r}191 \\
\text { Aug. }\end{array}$ & $\begin{array}{r}13 \\
7.2 \\
8.2 \\
11.3 \\
14.4 \\
15.4\end{array}$ & $\begin{array}{l}\mathrm{P} \\
\mathrm{Ep} \\
\mathrm{P} \\
\mathrm{P} \\
\mathrm{P}\end{array}$ & W & $\begin{array}{l}\text { I } \\
\text { I } \\
\text { I } \\
\text { I }\end{array}$ & $\begin{array}{ccc}\mathrm{h} & \mathrm{m} & \mathrm{s} \\
13 & 41 & 37.29 \\
14 & 36 & 40.70 \\
17 & 33 & \\
20 & 25 & 12.62 \\
21 & 15 & 58.58\end{array}$ & $\begin{array}{r}\mathrm{s} \\
+0.75 \\
+.74 \\
+.76 \\
+.80\end{array}$ & $\begin{array}{l}\mathrm{s} \\
-0.13 \\
-.19 \\
-.06 \\
+.03\end{array}$ & $\begin{array}{r}\prime \prime \\
-1.8 \\
-2.6 \\
-1.1 \\
+.1\end{array}$ & $\begin{array}{rr}N & \mathbf{S} \\
\mathbf{S} \\
\mathbf{S}\end{array}$ & \begin{tabular}{rrr}
$\circ$ & $\prime$ & \multicolumn{1}{c}{} \\
-13 & 38 & \\
-19 & 23 & \\
-28 & 30 & 16.4 \\
-23 & 27 & 29.4 \\
-19 & 19 & 6.4
\end{tabular} & $\begin{array}{l}-0.4 \\
+2.4 \\
+2.6\end{array}$ & $\begin{array}{r}-0.6 \\
-.7 \\
-1.0\end{array}$ & $\begin{array}{r}-0.6 \\
-. .5 \\
-1.2\end{array}$ \\
\hline & $\begin{array}{l}17.5 \\
19.6 \\
20.6 \\
21.7 \\
23.7\end{array}$ & $\begin{array}{l}\mathrm{Ep} \\
\mathrm{P} \\
\mathrm{P} \\
\mathrm{P} \\
\mathrm{P}\end{array}$ & & $\begin{array}{l}\text { II } \\
\text { II } \\
\text { II } \\
\text { II } \\
\text { II }\end{array}$ & $\begin{array}{rrr}22 & 48 & 19.53 \\
0 & 14 & 15.15 \\
0 & 57 & 24.70 \\
1 & 42 & 11.28 \\
3 & 21 & 3.67\end{array}$ & $\begin{array}{l}+.63 \\
+.44 \\
+.42 \\
+.45 \\
+.74\end{array}$ & $\begin{array}{l}-.07 \\
=.23 \\
=.26 \\
=.25 \\
-.04\end{array}$ & $\begin{array}{l}-.9 \\
-3.1 \\
-3.8 \\
-3.7 \\
-.7\end{array}$ & $\begin{array}{l}\mathrm{N} \\
\mathrm{N} \\
\mathrm{N} \\
\mathrm{N} \\
\mathrm{N}\end{array}$ & $\begin{array}{rrr}-8 & 59 & 22.2 \\
+\quad 2 & 32 & 39.6 \\
+8 & 17 & 24.9 \\
+13 & 47 & 0.1 \\
+23 & 828.7\end{array}$ & $\begin{array}{l}+4.8 \\
+5.0 \\
+4.5 \\
+3.9 \\
+2.9\end{array}$ & $\begin{array}{r}+.4 \\
+.3 \\
-.2 \\
-.4 \\
.0\end{array}$ & $\begin{array}{l}+.6 \\
+1.5 \\
+1.3 \\
+.9 \\
+.1\end{array}$ \\
\hline Sept. & $\begin{array}{r}4.2 \\
5.2 \\
8.3 \\
9.3 \\
10.3\end{array}$ & $\begin{array}{l}\mathbf{P} \\
\mathbf{M} \\
\mathbf{M} \\
\mathbf{M}\end{array}$ & & $\begin{array}{l}\text { I } \\
\text { I } \\
\text { I } \\
\text { I } \\
\text { I }\end{array}$ & $\begin{array}{rrr}14 & 14 & 48.03 \\
15 & 12 & 55.38 \\
18 & 14 & 42.21 \\
19 & 13 & 15.42 \\
20 & 8 & 32.41\end{array}$ & $\begin{array}{l}+.74 \\
+.60 \\
+.91 \\
+.84 \\
+.82\end{array}$ & $\begin{array}{l}-.14 \\
=.33 \\
=.03 \\
=.06 \\
-.03\end{array}$ & $\begin{array}{l}-1.9 \\
-4.4 \\
-.5 \\
-1.0 \\
-.4\end{array}$ & $\begin{array}{l}\mathbf{S} \\
\mathbf{S} \\
\mathbf{S}\end{array}$ & $\begin{array}{rrr}-17 & 22 & \\
-22 & 34 & \\
-28 & 38 & 4.2 \\
-27 & 19 & 32.0 \\
-24 & 37 & 0.4\end{array}$ & $\begin{array}{l}\overline{+} .1 \\
+1.5 \\
+3.5\end{array}$ & $\begin{array}{r}-1.3 \\
-.7 \\
+.5\end{array}$ & $\begin{array}{r}-1.3 \\
-.6 \\
+.5\end{array}$ \\
\hline Oct. & $\begin{array}{r}11.4 \\
22.7 \\
23.7 \\
24.7 \\
4.2\end{array}$ & $\begin{array}{l}\mathrm{M} \\
\mathrm{P} \\
\mathrm{P} \\
\mathrm{P}\end{array}$ & & $\begin{array}{ll}\text { I } & \\
& \text { II } \\
& \text { II }\end{array}$ & $\begin{array}{rrr}21 & 0 & 8.36 \\
5 & 55 & 30.88 \\
6 & 58 & \\
8 & 1 & 27.22 \\
16 & 49 & 34.83\end{array}$ & $\begin{array}{l}+.84 \\
+.91 \\
+.78 \\
+.86\end{array}$ & $\begin{array}{l}+.04 \\
+.09 \\
+.01 \\
-.08\end{array}$ & $\begin{array}{l}+.6 \\
+1.1 \\
+.1 \\
-1.1\end{array}$ & $N_{S}^{S}$ & $\begin{array}{rrr}-20 & 47 & 2.7 \\
+28 & 43 & 23.5 \\
+27 & 48 & 30.2 \\
+25 & 3 & \\
-27 & 42 & \end{array}$ & $\begin{array}{r}+4.1 \\
+.3 \\
+.5\end{array}$ & $\begin{array}{l}+.4 \\
+\quad .2 \\
+2.3\end{array}$ & $\begin{array}{r}+.1 \\
+.2 \\
+2.3\end{array}$ \\
\hline & $\begin{array}{r}6.2 \\
9.3 \\
12.4 \\
13.4 \\
14.5\end{array}$ & $\begin{array}{l}\mathrm{Ep} \\
\mathrm{Ep} \\
\mathrm{P} \\
\mathrm{Ep}\end{array}$ & & $\begin{array}{ll}\text { I } & \\
\text { I } & \\
\text { I } \\
\text { I } \\
\text { I } & \text { II }\end{array}$ & $\begin{array}{rrr}18 & 53 & 3.45 \\
21 & 32 & 44.49 \\
23 & 45 & 52.99 \\
0 & 28 & 45.92 \\
1 & 12 & 38.72\end{array}$ & $\begin{array}{l}+.95 \\
+.86 \\
+.71 \\
+.70 \\
+.62\end{array}$ & $\begin{array}{l}+.06 \\
+.10 \\
+.01 \\
+.01 \\
-.11\end{array}$ & $\begin{array}{l}+.7 \\
+1.3 \\
-.3 \\
-.3 \\
-2.1\end{array}$ & $\begin{array}{r}\mathrm{S} \\
\mathrm{S} \\
\mathrm{S} \\
\mathrm{S} \\
\mathrm{S} \\
\mathrm{S}\end{array}$ & $\begin{array}{rrr}-27 & 56 & 7.2 \\
-17 & 42 & 20.7 \\
-1 & 18 & 55.0 \\
+4 & 31 & 16.9 \\
+10 & 14 & 5.2\end{array}$ & $\begin{array}{l}+1.7 \\
+4.1 \\
+4.7 \\
+5.1 \\
+3.9\end{array}$ & $\begin{array}{l}-.2 \\
=.1 \\
=.7 \\
-.3 \\
-1.2\end{array}$ & $\begin{array}{l}-.3 \\
=.6 \\
=.7 \\
=.3 \\
-.6\end{array}$ \\
\hline Nov. & $\begin{array}{r}15.5 \\
16.5 \\
21.7 \\
22.7 \\
5.3\end{array}$ & $\begin{array}{l}\mathbf{M} \\
\mathbf{P} \\
\mathbf{E p} \\
\mathbf{M} \\
\mathbf{P}\end{array}$ & $\begin{array}{l}\text { W } \\
\mathrm{E}\end{array}$ & $\begin{array}{ll}\text { II } \\
\text { II } \\
\text { II } \\
\text { II }\end{array}$ & $\begin{array}{rrr}1 & 58 & 32.64 \\
2 & 47 & 23.33 \\
7 & 38 & 43.04 \\
8 & 38 & 43.71 \\
21 & 14 & 36.66\end{array}$ & $\begin{array}{l}+.56 \\
+.59 \\
+.83 \\
+.70 \\
+.78\end{array}$ & $\begin{array}{l}-.19 \\
\overline{-} .18 \\
+.07 \\
+.02 \\
+.03\end{array}$ & $\begin{array}{r}-2.6 \\
-2.5 \\
+.5 \\
+.6 \\
+.2\end{array}$ & $\begin{array}{ll}\mathrm{N} & \\
\mathbf{N} & \\
& \mathbf{S} \\
& \mathbf{S} \\
& \mathrm{S}\end{array}$ & $\begin{array}{rrr}+15 & 37 & 5.2 \\
+20 & 25 & 44.3 \\
+26 & 9 & 17.3 \\
+22 & 25 & 17.8 \\
-19 & 17 & 52.4\end{array}$ & $\begin{array}{l}+4.9 \\
+4.1 \\
+.7 \\
+1.7 \\
+3.2\end{array}$ & $\begin{array}{l}+.2 \\
+.2 \\
+3.1 \\
+1.7 \\
-.6\end{array}$ & $\begin{array}{l}+1.0 \\
+1.0 \\
+3.2 \\
+1.6 \\
-.7\end{array}$ \\
\hline & $\begin{array}{r}6.3 \\
7.3 \\
10.3 \\
12.5 \\
13.5\end{array}$ & $\begin{array}{l}\mathbf{P} \\
\mathbf{M} \\
\mathbf{P} \\
\mathbf{E p} \\
\mathbf{P}\end{array}$ & & $\begin{array}{ll}\text { I } \\
\text { I } \\
\text { I } \\
\text { I } \\
\text { I } & \text { II }\end{array}$ & $\begin{array}{rrr}22 & 2 & 12.60 \\
22 & 47 & 2.65 \\
0 & 56 & 31.73 \\
2 & 29 & 57.44 \\
3 & 21 & 45.13\end{array}$ & $\begin{array}{l}+.74 \\
+.79 \\
+.64 \\
+.86 \\
+.76\end{array}$ & $\begin{array}{l}+.03 \\
+.09 \\
.09 \\
+.08 \\
-.04\end{array}$ & $\begin{array}{l}+.5 \\
+1.4 \\
-1.2 \\
+.5 \\
-.6\end{array}$ & $\begin{array}{ll} & S \\
& S \\
& S \\
N & S \\
N & \end{array}$ & $\begin{array}{rrr}-14 & 23 & 35.0 \\
-9 & 0 & 1.8 \\
+8 & 14 & 38.2 \\
+18 & 48 & 23.7 \\
+23 & 6 & 11.5\end{array}$ & $\begin{array}{l}+5.0 \\
+5.5 \\
+5.7 \\
+3.2 \\
+3.8\end{array}$ & $\begin{array}{l}+.7 \\
+.7 \\
+.2 \\
-1.3 \\
+.2\end{array}$ & $\begin{array}{r}+.4 \\
.0 \\
+.6 \\
+1.7 \\
+.3\end{array}$ \\
\hline & $\begin{array}{l}14.5 \\
17.6 \\
18.7 \\
20.8 \\
21.8\end{array}$ & $\begin{array}{l}\mathbf{P} \\
\mathbf{E p} \\
\mathbf{P} \\
\text { Ep } \\
\mathbf{P}\end{array}$ & & $\begin{array}{l}\text { II } \\
\text { II } \\
\text { II } \\
\text { II }\end{array}$ & $\begin{array}{rrr}4 & 17 & 33.47 \\
7 & 20 & 13.24 \\
8 & 20 & 19.09 \\
10 & 13 & \\
11 & 5 & 28.80\end{array}$ & $\begin{array}{l}+.79 \\
+.86 \\
+.62 \\
+.54\end{array}$ & $\begin{array}{l}\bar{t} .03 \\
\pm .10 \\
-.09 \\
-.11\end{array}$ & $\begin{array}{l}-.5 \\
+1.1 \\
-1.0 \\
-.9\end{array}$ & $\begin{array}{rr}\mathbf{N} \\
\mathbf{S} \\
\mathbf{S} \\
\mathbf{S} \\
\mathbf{S}\end{array}$ & 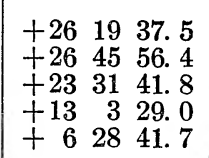 & $\begin{array}{r}+2.4 \\
.0 \\
-3.1 \\
-5.3 \\
-5.8\end{array}$ & $\begin{array}{l}+.1 \\
+1.8 \\
-.3 \\
-1.2 \\
-1.4\end{array}$ & $\begin{array}{l}+.2 \\
+2.0 \\
-.5 \\
-1.1 \\
-1.8\end{array}$ \\
\hline Dec. & $\begin{array}{r}4.2 \\
5.2 \\
8.3 \\
9.4 \\
11.4\end{array}$ & $\begin{array}{l}\mathrm{Ep} \\
\mathrm{Ep} \\
\mathrm{M} \\
\mathrm{M} \\
\mathbf{P}\end{array}$ & & $\begin{array}{l}\text { I } \\
\text { I } \\
\text { I } \\
\text { I } \\
\text { I }\end{array}$ & $\begin{array}{rrr}22 & 28 & 49.39 \\
23 & 12 & 48.59 \\
1 & 23 & 0.99 \\
2 & 9.52 .01 \\
3 & 54 & 57.52\end{array}$ & $\begin{array}{l}+.79 \\
+.68 \\
+.76 \\
+.74 \\
+.68\end{array}$ & $\begin{array}{r}+.08 \\
-.02 \\
.00 \\
-.06 \\
-.18\end{array}$ & $\begin{array}{l}+.9 \\
-.3 \\
-.3 \\
-.9 \\
-2.5\end{array}$ & $\begin{aligned} & \mathbf{S} \\
& \mathbf{S} \\
& \mathrm{S} \\
& \mathrm{N} \\
& \mathrm{N} \\
& \mathrm{S}\end{aligned}$ & 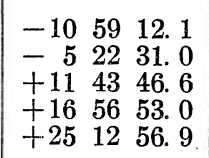 & $\begin{array}{l}+4.2 \\
+5.1 \\
+4.8 \\
+5.0 \\
+2.7\end{array}$ & $\begin{array}{r}-.3 \\
+.2 \\
-.6 \\
.0 \\
-.3\end{array}$ & $\begin{array}{l}-.8 \\
+.3 \\
+.7 \\
+.2 \\
+.2\end{array}$ \\
\hline 191 & $\begin{array}{l}13.5 \\
15.6 \\
18.7 \\
19.7 \\
4\end{array}$ & $\begin{array}{l}\text { M } \\
\text { Ep } \\
\text { Ep } \\
\text { Ep }\end{array}$ & & $\begin{array}{l}\text { II } \\
\text { II } \\
\text { II } \\
\text { II }\end{array}$ & $\begin{array}{rrr}5 & 55 & 55.74 \\
8 & 1 & 2.32 \\
10 & 49 & 22.31 \\
11 & 41 & 1.82\end{array}$ & $\begin{array}{l}+.85 \\
+.79 \\
+.61 \\
+.59\end{array}$ & $\begin{array}{r}.00 \\
+.06 \\
-.00 \\
-.03\end{array}$ & $\begin{array}{r}.0 \\
+\quad .6 \\
+\quad .9 \\
.0\end{array}$ & $\begin{array}{l}\mathbf{N} \\
\underset{S}{S} \\
\mathbf{S}\end{array}$ & $\begin{array}{l}+282225.2 \\
+243255.2 \\
+81527.6 \\
+12553.2\end{array}$ & $\begin{array}{l}+.7 \\
-1.0 \\
-6.2 \\
-4.7\end{array}$ & $\begin{array}{l}+.6 \\
+1.2 \\
-2.5 \\
-.9\end{array}$ & $\begin{array}{l}+.6 \\
+1.4 \\
-2.2 \\
-.9\end{array}$ \\
\hline Jan. & $\begin{array}{r}5.3 \\
6.3 \\
7.3 \\
8.4 \\
10.5\end{array}$ & $\begin{array}{l}\mathrm{Ep} \\
\mathrm{M} \\
\mathrm{P} \\
\mathrm{Ep}\end{array}$ & $\mathrm{E}$ & $\begin{array}{l}\text { I } \\
\text { I } \\
\text { I } \\
\text { I } \\
\text { I }\end{array}$ & $\begin{array}{llr}1 & 48 & 56.78 \\
2 & 37 & 15.63 \\
3 & 29 & 35.41 \\
4 & 26 & 27.01 \\
6 & 31 & 3.21\end{array}$ & $\begin{array}{l}+.87 \\
+.94 \\
+.93 \\
+.95 \\
+.96\end{array}$ & $\begin{array}{l}+.06 \\
+.08 \\
+.03 \\
+.02 \\
+.07\end{array}$ & $\begin{array}{r}+.4 \\
+.9 \\
+.0 \\
+.1 \\
+\quad .8\end{array}$ & $\begin{array}{ll} & \mathbf{S} \\
& \mathbf{S} \\
\mathrm{N} & \stackrel{\mathbf{S}}{\mathbf{S}} \\
\mathrm{N} & \end{array}$ & $\begin{array}{rrr}+14 & 58 & 56.4 \\
+19 & 48 & 33.4 \\
+23 & 51 & 40.3 \\
+26 & 48 & 38.5 \\
+28 & 2 & 2.4\end{array}$ & $\begin{array}{l}+4.6 \\
+4.4 \\
+2.4 \\
+1.8 \\
+\quad .7\end{array}$ & $\begin{array}{l}-.7 \\
=.1 \\
-1.2 \\
-.6 \\
+1.3\end{array}$ & $\begin{array}{l}-1.0 \\
-. .5 \\
-1.4 \\
-.7 \\
+1.4\end{array}$ \\
\hline
\end{tabular}




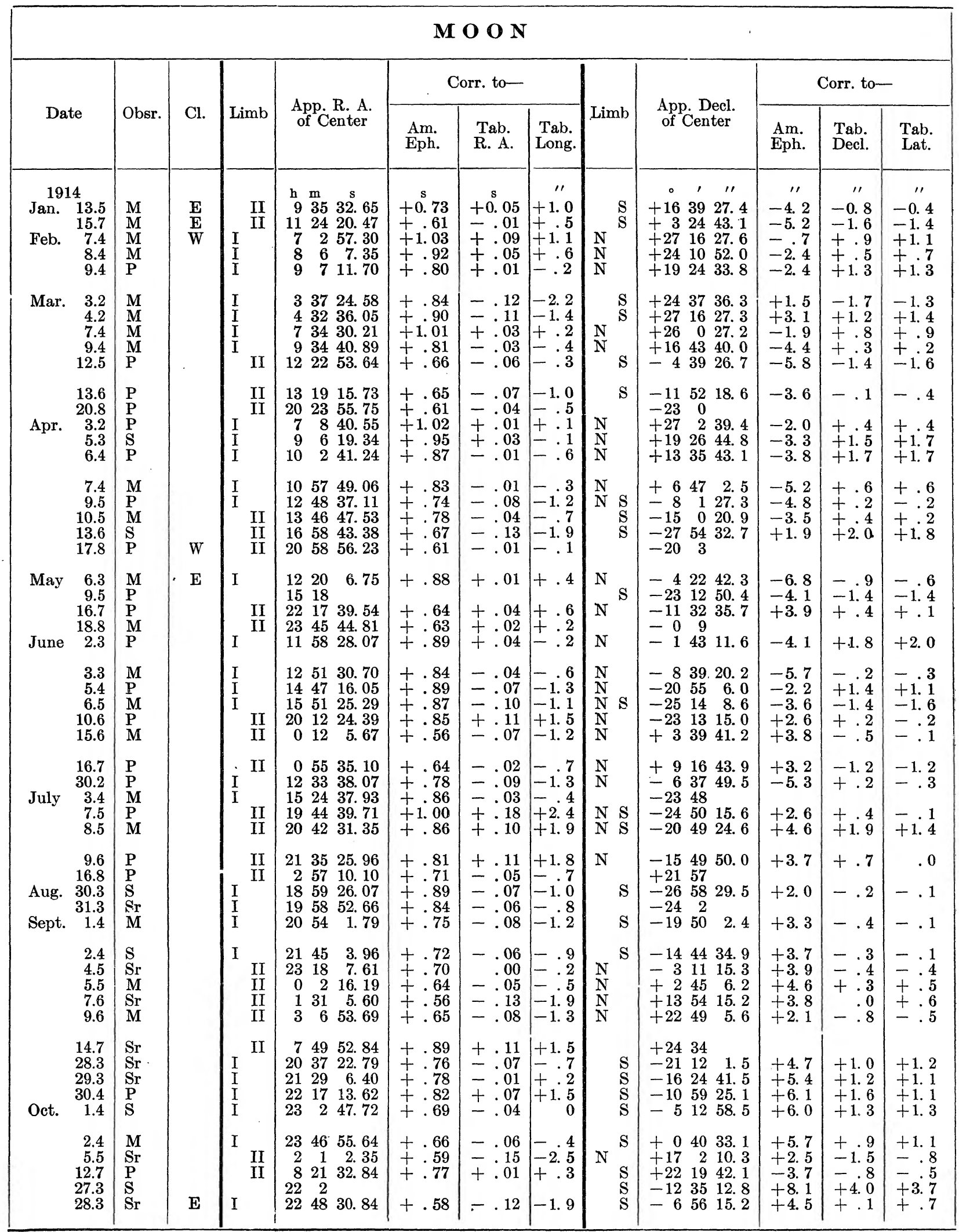




\begin{tabular}{|c|c|c|c|c|c|c|c|c|c|c|c|c|c|}
\hline \multicolumn{14}{|c|}{$\mathbf{M O O N}$} \\
\hline \multirow{2}{*}{\multicolumn{2}{|c|}{ Date }} & \multirow[b]{2}{*}{ Obsr. } & \multirow[b]{2}{*}{ Cl. } & \multirow[b]{2}{*}{ Limb } & \multirow{2}{*}{ App. R. A. } & \multicolumn{3}{|c|}{ Corr. to- } & \multirow[b]{2}{*}{ Limb } & \multirow{2}{*}{$\begin{array}{l}\text { App. Decl. } \\
\text { of Center }\end{array}$} & \multicolumn{3}{|c|}{ Corr. to- } \\
\hline & & & & & & $\begin{array}{l}\text { Am. } \\
\text { Eph. }\end{array}$ & $\begin{array}{l}\text { Tab. } \\
\text { R. A. }\end{array}$ & $\begin{array}{l}\text { Tab. } \\
\text { Long. }\end{array}$ & & & $\begin{array}{l}\text { Am. } \\
\text { Eph. }\end{array}$ & $\begin{array}{l}\text { Tab. } \\
\text { Decl. }\end{array}$ & $\begin{array}{l}\text { Tab. } \\
\text { Lat. }\end{array}$ \\
\hline $\begin{array}{l}1914 \\
\text { Oct. } \\
\text { Nov. }\end{array}$ & $\begin{array}{l}14 \\
30.4 \\
31.4 \\
23.2 \\
24.3 \\
25.3\end{array}$ & $\begin{array}{l}\mathrm{S} \\
\mathrm{Sr} \\
\mathrm{P} \\
\mathrm{S} \\
\mathrm{Sr}\end{array}$ & $\frac{\mathrm{E}}{\mathrm{E}}$ & $\begin{array}{l}\text { I } \\
\text { I } \\
\text { I } \\
\text { I } \\
\text { I }\end{array}$ & $\begin{array}{rrc}\mathrm{h} & \mathrm{m} & \mathrm{s} \\
0 & 16 & 21.00 \\
1 & 0 & 18.38 \\
21 & 44 & 36.48 \\
22 & 32 & 31.63 \\
23 & 17 & 44.75\end{array}$ & $\begin{array}{r}\mathrm{s} \\
+0.70 \\
+.65 \\
+.87 \\
+.74 \\
+.55\end{array}$ & $\begin{array}{l}\mathrm{s} \\
-0.01 \\
-.07 \\
+.16 \\
+.05 \\
-.12\end{array}$ & $\begin{array}{r}11 \\
+0.6 \\
-1.0 \\
+2.6 \\
+1.0 \\
-1.7\end{array}$ & $\begin{array}{l}\mathrm{S} \\
\mathrm{S} \\
\mathrm{S} \\
\mathrm{S}\end{array}$ & 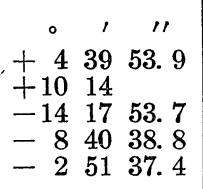 & $\begin{array}{r}\text { 'l } \\
+6.6 \\
+4.9 \\
+4.6 \\
+3.9\end{array}$ & $\begin{array}{r}11 \\
+1.8 \\
+1.2 \\
+.7 \\
-.4\end{array}$ & $\begin{array}{r}\prime \prime \\
+1.6 \\
+\quad .3 \\
+.2 \\
+.2\end{array}$ \\
\hline Dec. & $\begin{array}{l}28.4 \\
21.2 \\
22.2 \\
23.2 \\
30.4\end{array}$ & $\begin{array}{l}\mathrm{Sr} \\
\mathrm{Sr} \\
\mathrm{S} \\
\mathrm{Sr} \\
\mathrm{P}\end{array}$ & & $\begin{array}{l}\mathrm{I} \\
\mathrm{I} \\
\mathrm{I} \\
\mathrm{I}\end{array}$ & $\begin{array}{rrr}1 & 30 & 7.28 \\
22 & 12 & \\
22 & 59 & 20.94 \\
23 & 44 & 26.81 \\
5 & 29 & 55.60\end{array}$ & $\begin{array}{l}+.67 \\
+.74 \\
+.72 \\
+.97\end{array}$ & $\begin{array}{l}-.05 \\
+.03 \\
+.01 \\
+.11\end{array}$ & $\begin{array}{l}-.2 \\
+1.3 \\
+.6 \\
+1.4\end{array}$ & 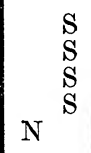 & $\begin{array}{rrr}+13 & 50 & 59.9 \\
-10 & 52 & 35.3 \\
-4 & 57 & 44.6 \\
+\quad 0 & 58 & 34.4 \\
+27 & 52 & 35.6\end{array}$ & $\begin{array}{l}+5.7 \\
+4.4 \\
+6.2 \\
+5.0 \\
+\quad .1\end{array}$ & $\begin{array}{l}+1.2 \\
+.6 \\
+2.2 \\
+.7 \\
-1.1\end{array}$ & $\begin{array}{l}+1.3 \\
+.5 \\
+1.7 \\
+.5 \\
-1.2\end{array}$ \\
\hline $\begin{array}{l}191 \\
\text { Jan. }\end{array}$ & $\begin{array}{r}15 \\
1.5 \\
4.6 \\
7.7 \\
9.8 \\
21.2\end{array}$ & $\begin{array}{l}\mathrm{M} \\
\mathrm{S} \\
\mathrm{Sr} \\
\mathrm{M} \\
\mathrm{P}\end{array}$ & & $\mathrm{I}^{\text {II }}$ & $\begin{array}{rrr}7 & 25 & \\
10 & 5 & 38.06 \\
12 & 36 & 4.94 \\
14 & 26 & 21.31 \\
0 & 54 & 21.19\end{array}$ & $\begin{array}{l}+.66 \\
+.70 \\
+.64 \\
+.84\end{array}$ & $\begin{array}{l}+.02 \\
+.06 \\
.07 \\
+.05\end{array}$ & $\begin{array}{l}-.6 \\
+.8 \\
-1.1 \\
+1.1\end{array}$ & $\begin{array}{l}\mathrm{N} \\
\mathrm{S} \\
\mathrm{S} \\
\mathrm{S}\end{array}$ & $\begin{array}{rrr}+25 & 12 & 39.7 \\
+11 & 30 & 59.3 \\
-7 & 49 & \\
-19 & 44 & 14.8 \\
+10 & 9 & 13.1\end{array}$ & $\begin{array}{l}-2.0 \\
-.8 \\
-2.4 \\
+5.5\end{array}$ & $\begin{array}{l}-.8 \\
+2.4 \\
+.2 \\
+1.0\end{array}$ & $\begin{array}{l}\overline{+} .8 \\
-.5 \\
+.5\end{array}$ \\
\hline Feb. & $\begin{array}{r}26.4 \\
28.4 \\
29.4 \\
8.8 \\
18.2\end{array}$ & $\begin{array}{l}\mathrm{M} \\
\mathrm{S} \\
\mathrm{M} \\
\mathrm{P} \\
\mathrm{P}\end{array}$ & $\begin{array}{l}\mathrm{W} \\
\mathrm{E}\end{array}$ & $\begin{array}{l}\text { I } \\
\text { I } \\
\text { I }\end{array}$ & $\begin{array}{rrr}5 & 6 & 22.17 \\
7 & 1 & 48.49 \\
7 & 58 & 50.34 \\
17 & 16 & 53.68 \\
1 & 20 & 11.27\end{array}$ & $\begin{array}{l}+1.04 \\
+1.19 \\
+.96 \\
+.66 \\
+.81\end{array}$ & $\begin{array}{l}+.11 \\
+.29 \\
+.11 \\
+.03 \\
+.01\end{array}$ & $\begin{array}{l}+1.6 \\
+3.9 \\
+1.6 \\
+\quad .4 \\
+\quad .2\end{array}$ & ${\underset{N}{N}}^{S}$ & $\begin{array}{lll}+27 & 46 & 46.3 \\
+26 & 16 & 34.9 \\
+23 & 16 & 57.4 \\
+27 & 55 & \\
+13 & 14 & 16.1\end{array}$ & $\begin{array}{l}+2.6 \\
-2.0 \\
-2.6 \\
+4.6\end{array}$ & $\begin{array}{l}+1.2 \\
-1.0 \\
-.4 \\
+.2\end{array}$ & $\begin{array}{r}+1.0 \\
-.6 \\
.0 \\
+.1\end{array}$ \\
\hline Mar. & $\begin{array}{r}19.2 \\
20.2 \\
26.4 \\
27.5 \\
1.5\end{array}$ & $\begin{array}{l}\mathrm{S} \\
\mathrm{Sr} \\
\mathrm{P} \\
\mathrm{Sr} \\
\mathrm{Sr}\end{array}$ & & $\begin{array}{ll}\text { I } & \\
\text { I } & \\
I & \\
& \text { II }\end{array}$ & $\begin{array}{rrr}2 & 7 & 16.37 \\
2 & 56 & 23.71 \\
8 & 29 & \\
9 & 23 & 9.25 \\
11 & 7 & 57.05\end{array}$ & $\begin{array}{l}+.84 \\
+.88 \\
+.85 \\
+.79\end{array}$ & $\begin{array}{l}-.01 \\
-.02 \\
+.01 \\
+.04\end{array}$ & $\begin{array}{l}+.4 \\
-.2 \\
-.2 \\
+\quad .8\end{array}$ & 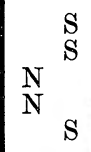 & 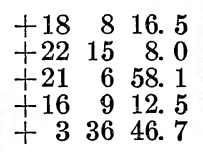 & $\begin{array}{l}+6.0 \\
+3.7 \\
-2.5 \\
-3.1 \\
-5.7\end{array}$ & $\begin{array}{l}+1.9 \\
+.1 \\
+.7 \\
+1.0 \\
-1.0\end{array}$ & $\begin{array}{l}+1.7 \\
+.1 \\
+.7 \\
+1.1 \\
+.6\end{array}$ \\
\hline & $\begin{array}{r}3.6 \\
8.8 \\
9.8 \\
24.3 \\
26.4\end{array}$ & $\begin{array}{l}\mathrm{P} \\
\mathrm{Sr} \\
\mathrm{S} \\
\mathrm{Sr} \\
\mathrm{Sr}\end{array}$ & & $\mathrm{I}_{\text {I }}$ & $\begin{array}{rrr}12 & 53 & 34.59 \\
18 & 2 & 57.86 \\
19 & 6 & 53.60 \\
7 & 7 & 26.82 \\
8 & 57 & 0.28\end{array}$ & $\begin{array}{l}+.67 \\
+.61 \\
+.66 \\
+1.08 \\
+.83\end{array}$ & $\begin{array}{l}-.06 \\
\overline{0} .05 \\
+.03 \\
+.03 \\
-.06\end{array}$ & $\begin{array}{l}-.5 \\
-.7 \\
+.4 \\
+1.2 \\
-1.1\end{array}$ & $\begin{array}{l}\mathrm{N} \\
\mathrm{N}\end{array}$ & $\begin{array}{rrr}-10 & 5 & 44.4 \\
-27 & 48 & \\
-26 & 4 & \\
+25 & 57 & 44.0 \\
+18 & 37 & 3.7\end{array}$ & $\begin{array}{l}-1.7 \\
-3.1\end{array}$ & $\begin{array}{l}-1.0 \\
+\quad .1 \\
+1.0\end{array}$ & $\begin{array}{l}-1.2 \\
\\
+.3 \\
+\quad .8\end{array}$ \\
\hline Apr. & $\begin{array}{r}27.4 \\
29.4 \\
30.5 \\
31.5 \\
4.7\end{array}$ & $\begin{array}{l}\mathrm{Sr} \\
\mathrm{M} \\
\mathrm{P} \\
\mathrm{S} \\
\mathrm{Sr}\end{array}$ & & $\begin{array}{ll}\text { I } & \\
\text { I } & \\
\text { I } & \text { II } \\
& \text { II } \\
& \text { II }\end{array}$ & $\begin{array}{rrr}9 & 49 & 50.97 \\
11 & 34 & 15.53 \\
12 & 27 & 53.96 \\
13 & 24 & 6.31 \\
17 & 41 & 54.13\end{array}$ & $\begin{array}{l}+.78 \\
+.80 \\
+.77 \\
+.75 \\
+.60\end{array}$ & $\begin{array}{l}\bar{T} .06 \\
+.01 \\
\overline{.} .04 \\
-.03 \\
-.14\end{array}$ & $\begin{array}{l}-1.6 \\
-.2 \\
-.2 \\
-1.2 \\
-1.8\end{array}$ & $\begin{array}{ll}\mathrm{N} & \\
\mathrm{N} & \\
& \mathrm{S} \\
& \mathrm{S} \\
\mathrm{S}\end{array}$ & $\begin{array}{rrr}+13 & 12 & 34.4 \\
+ & 0 & 53.3 \\
-6 & 51 & 39.5 \\
-13 & 34 & 49.4 \\
-27 & 48 & 15.2\end{array}$ & $\begin{array}{l}-2.7 \\
-4.8 \\
-6.0 \\
-2.3 \\
+.6\end{array}$ & $\begin{array}{l}+2.2 \\
+.6 \\
+1.2 \\
+1.7 \\
-.3\end{array}$ & $\begin{array}{l}+1.8 \\
+.7 \\
-1.2 \\
+1.5 \\
-.3\end{array}$ \\
\hline June & $\begin{array}{r}7.8 \\
8.8 \\
21.2 \\
24.3 \\
17.2\end{array}$ & $\begin{array}{l}\mathrm{P} \\
\mathrm{M} \\
\mathrm{S} \\
\mathrm{S} \\
\mathrm{Sr}\end{array}$ & $\underset{\mathrm{W}}{\mathrm{E}}$ & $\mathrm{I}_{\text {I }}{ }^{\text {II }}$ & $\begin{array}{rrr}20 & 46 & 24.13 \\
21 & 38 & 51.02 \\
7 & 40 & 20.40 \\
10 & 16 & 37.22 \\
9 & 39 & 47.93\end{array}$ & $\begin{array}{l}+.64 \\
+.69 \\
+.95 \\
+.82 \\
+.78\end{array}$ & $\begin{array}{l}+.03 \\
\pm .09 \\
\pm .03 \\
-.03 \\
-.15\end{array}$ & $\begin{array}{l}+.4 \\
+1.2 \\
-\quad .2 \\
-\quad .9 \\
-2.1\end{array}$ & $\stackrel{N}{N}$ & $\begin{array}{r}-1929 \\
-1422 \\
+24636.8 \\
+95358.0 \\
+1335\end{array}$ & $\begin{array}{l}-4.5 \\
-4.2\end{array}$ & $\begin{array}{l}-1.9 \\
+1.3\end{array}$ & $\begin{array}{r}-1.9 \\
+1.2\end{array}$ \\
\hline & $\begin{array}{l}19.2 \\
22.3 \\
23.3 \\
24.4 \\
25.4\end{array}$ & $\begin{array}{l}M \\
P \\
M \\
S \\
P\end{array}$ & & $\begin{array}{l}\mathrm{I} \\
\mathrm{I} \\
\mathrm{I} \\
\mathrm{I}\end{array}$ & $\begin{array}{rrr}11 & 16 & 45.07 \\
13 & 53 & 44.18 \\
14 & 54 & 36.62 \\
16 & 0 & 52.08 \\
17 & 11 & \end{array}$ & $\begin{array}{l}+.85 \\
+.92 \\
+.90 \\
+.89\end{array}$ & $\begin{array}{l}-.07 \\
=.03 \\
=.06 \\
-.05\end{array}$ & $\begin{array}{l}-.8 \\
-.9 \\
-.9 \\
-.4\end{array}$ & $\begin{array}{lr}\mathrm{N} & \\
\mathrm{N} & \\
\mathrm{N} & \\
\mathrm{N} & \\
& \end{array}$ & $\begin{array}{rrr}+1 & 47 & 15.1 \\
-16 & 56 & 10.2 \\
-22 & 1 & 38.9 \\
-25 & 41 & 19.0 \\
-27 & 23 & 50.9\end{array}$ & $\begin{array}{l}-7.0 \\
-3.6 \\
-3.1 \\
-3.7 \\
-2.1\end{array}$ & $\begin{array}{l}-.8 \\
+1.0 \\
+.2 \\
-1.9 \\
-1.7\end{array}$ & $\begin{array}{l}-1.0 \\
+.9 \\
-1.0 \\
-1.9\end{array}$ \\
\hline July & $\begin{array}{r}26.5 \\
28.5 \\
2.7 \\
22.4 \\
23.4\end{array}$ & $\begin{array}{l}\mathrm{P} \\
\mathrm{M} \\
\mathrm{P} \\
\mathrm{Sr} \\
\mathrm{M}\end{array}$ & & $\begin{array}{ll}\text { I } & \text { II } \\
& \text { II } \\
& \text { II } \\
\text { I } & \\
\text { I } & \end{array}$ & $\begin{array}{rrr}18 & 22 & 4.92 \\
20 & 33 & 7.26 \\
23 & 58 & 14.68 \\
16 & 39 & 15.41 \\
17 & 48 & 29.09\end{array}$ & $\begin{array}{l}+.91 \\
+.81 \\
+.66 \\
+.93 \\
+1.09\end{array}$ & $\begin{array}{l}+.09 \\
+.14 \\
+.03 \\
+.10 \\
+.11\end{array}$ & $\begin{array}{l}+1.1 \\
+2.4 \\
+\quad .4 \\
-1.5 \\
+1.4\end{array}$ & $\begin{array}{ll} & \mathrm{S} \\
\mathrm{N} & \mathrm{S} \\
& \mathrm{S}\end{array}$ & $\begin{array}{rrr}-26 & 52 & 39.5 \\
-19 & 56 & 36.3 \\
+3 & 41 & \\
-26 & 58 & 31.3 \\
-27 & 27 & 2.4\end{array}$ & $\begin{array}{l}-1.4 \\
+4.1 \\
-.4 \\
-.3\end{array}$ & $\begin{array}{l}-2.2 \\
+1.9 \\
+.5 \\
-.8\end{array}$ & $\begin{array}{l}-2.2 \\
+1.4 \\
+.3 \\
-.8\end{array}$ \\
\hline $\begin{array}{l}\text { Aug. } \\
\text { Sept. }\end{array}$ & $\begin{array}{r}24.4 \\
31.7 \\
2.7 \\
14.2 \\
15.2\end{array}$ & $\begin{array}{l}\mathrm{S} \\
\mathrm{S} \\
\mathrm{S} \\
\mathrm{Sr} \\
\mathrm{P}\end{array}$ & W & $\begin{array}{ll}\text { I } & \\
& \text { II } \\
\text { I } & \text { II } \\
\text { I } & \end{array}$ & $\begin{array}{rrr}18 & 57 & 6.44 \\
1 & 11 & 38.40 \\
2 & 49 & 3.41 \\
15 & 56 & 31.58 \\
17 & 2 & 16.24\end{array}$ & $\begin{array}{l}+.96 \\
+.63 \\
+.59 \\
+.84 \\
+.87\end{array}$ & $\begin{array}{l} \pm .06 \\
\pm .02 \\
=.13 \\
=.14 \\
-.13\end{array}$ & $\begin{array}{l}+.7 \\
-.4 \\
-1.8 \\
-1.9 \\
-1.6\end{array}$ & $\mathrm{~N}^{\mathrm{S}}$ & $\begin{array}{lll}-25 & 47 & 59.2 \\
+12 & 43 & 33.0 \\
+21 & 47 & \\
-25 & 32 & \\
-27 & 17 & 46.6\end{array}$ & $\overline{+2.6}$ & $\begin{array}{l}-2.0 \\
-.6\end{array}$ & -2.1 \\
\hline
\end{tabular}




\begin{tabular}{|c|c|c|c|c|c|c|c|c|c|c|c|c|c|}
\hline \multicolumn{14}{|c|}{$\mathbf{M O O N}$} \\
\hline \multirow{2}{*}{\multicolumn{2}{|c|}{ Date }} & \multirow[b]{2}{*}{ Obsr. } & \multirow[b]{2}{*}{ Cl. } & \multirow[b]{2}{*}{ Limb } & \multirow{2}{*}{$\begin{array}{l}\text { App. R. A. } \\
\text { of Center }\end{array}$} & \multicolumn{3}{|c|}{ Corr. to- } & \multirow[b]{2}{*}{$\operatorname{Limb}$} & \multirow{2}{*}{ App. Decl. } & \multicolumn{3}{|c|}{ Corr. to- } \\
\hline & & & & & & Am. & $\begin{array}{l}\text { Tab. } \\
\text { R. A. }\end{array}$ & $\begin{array}{l}\text { Tab. } \\
\text { Long. }\end{array}$ & & & Am. & $\begin{array}{l}\text { Tab. } \\
\text { Decl. }\end{array}$ & $\begin{array}{l}\text { Tab. } \\
\text { Lat. }\end{array}$ \\
\hline $\begin{array}{l}1915 \\
\text { Sept. }\end{array}$ & $\begin{array}{l}5 \\
16.3 \\
20.4 \\
21.4 \\
22.4 \\
23.5\end{array}$ & $\begin{array}{l}\mathrm{S} \\
\mathrm{M} \\
\mathrm{Sr} \\
\mathrm{S} \\
\mathrm{S}\end{array}$ & $\mathrm{W}$ & $\begin{array}{ll}\text { I } & \\
\text { I } & \\
\text { I } & \\
\text { I } & \\
& \text { II }\end{array}$ & \begin{tabular}{rrr}
$\mathrm{h}$ & $\mathrm{m}$ & \multicolumn{1}{c}{$\mathrm{s}$} \\
18 & 8 & 40.52 \\
22 & 4 & 5.37 \\
22 & 54 & 11.57 \\
23 & 42 & 31.19 \\
0 & 30 & 10.58
\end{tabular} & $\begin{array}{r}\text { s } \\
+0.96 \\
+.73 \\
+.70 \\
+.66 \\
+.67\end{array}$ & $\begin{array}{c}\mathrm{s} \\
-0.03 \\
-.07 \\
-.08 \\
-.09 \\
-.06\end{array}$ & $\begin{array}{r}11 \\
-0.4 \\
-.7 \\
-.8 \\
-1.2 \\
-.8\end{array}$ & $\begin{array}{l}\stackrel{S}{S} \\
\stackrel{S}{S} \\
N\end{array}$ & 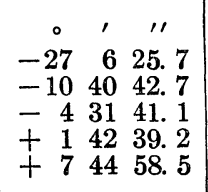 & $\begin{array}{l}11 \\
+0.5 \\
+5.4 \\
+5.0 \\
+4.4 \\
+4.2\end{array}$ & $\begin{array}{r}1 \prime \\
-0.9 \\
+.8 \\
+.4 \\
+.1 \\
.0\end{array}$ & $\begin{array}{r}11 \\
-0.9 \\
+1.0 \\
+\quad .6 \\
+.3 \\
+\quad .2\end{array}$ \\
\hline Oct. & $\begin{array}{l}24.5 \\
27.6 \\
28.7 \\
12.2 \\
15.3\end{array}$ & $\begin{array}{l}\mathrm{M} \\
\mathrm{Sr} \\
\mathrm{M} \\
\mathrm{Sr} \\
\mathrm{Sr}\end{array}$ & & $\begin{array}{ll} & \text { II } \\
& \text { II } \\
\text { II } & \\
\text { I } & \end{array}$ & $\begin{array}{rrr}1 & 18 & 9.27 \\
3 & 50 & 38.95 \\
4 & 44 & 49.20 \\
16 & 40 & 26.58 \\
19 & 56 & 17.57\end{array}$ & $\begin{array}{l}+.64 \\
+.72 \\
+.76 \\
+.76 \\
+.80\end{array}$ & $\begin{array}{l}-.09 \\
\overline{0} .02 \\
+.01 \\
-.14 \\
-.03\end{array}$ & $\begin{array}{r}-1.0 \\
-.3 \\
+.1 \\
-1.9 \\
-.6\end{array}$ & $\begin{array}{l}N \\
N \\
N\end{array}$ & $\begin{array}{rrr}+13 & 20 & 4.6 \\
+25 & 12 & 3.7 \\
+26 & 55 & 36.2 \\
-26 & 45 & \\
-22 & 23 & 24.2\end{array}$ & $\begin{array}{l}+4.4 \\
+1.6 \\
+.8 \\
+2.1\end{array}$ & $\begin{array}{l} \pm .5 \\
\pm .6 \\
-.7 \\
-1.0\end{array}$ & $\begin{array}{l} \pm .8 \\
-.6 \\
-.7 \\
-.9\end{array}$ \\
\hline & $\begin{array}{l}21.4 \\
22.5 \\
24.6 \\
25.6 \\
27.6\end{array}$ & $\begin{array}{l}\mathrm{M} \\
\mathrm{Sr} \\
\mathrm{S} \\
\mathrm{Sr} \\
\mathrm{P}\end{array}$ & $\mathrm{W}$ & $\begin{array}{ll}\text { I } & \\
\text { I } & \text { II } \\
& \text { II } \\
& \text { II }\end{array}$ & $\begin{array}{rrr}0 & 59 & 40.09 \\
1 & 48 & 2.30 \\
3 & 30 & 5.69 \\
4 & 23 & 49.16 \\
6 & 13 & \end{array}$ & $\begin{array}{l}+.72 \\
+.64 \\
+.65 \\
+.82\end{array}$ & $\begin{array}{l}-.05 \\
-.13 \\
-.13 \\
+.04\end{array}$ & $\begin{array}{l}-.6 \\
-1.9 \\
-2.1 \\
+.5\end{array}$ & $\begin{array}{l}N \\
N \\
N \\
N \\
N \\
N\end{array}$ & $\begin{array}{rrr}+11 & 14 & 16.8 \\
+16 & 21 & 23.1 \\
+24 & 230.0 \\
+26 & 13 & 29.7 \\
+26 & 40 & 52.7\end{array}$ & $\begin{array}{l}+4.4 \\
+3.5 \\
+1.3 \\
+1.5 \\
+.5\end{array}$ & $\begin{array}{r}.0 \\
-.6 \\
-1.6 \\
+.6 \\
+.3\end{array}$ & $\begin{array}{r}-.1 \\
-1.2 \\
-1.2 \\
+.7\end{array}$ \\
\hline Nov. & $\begin{array}{l}10.2 \\
13.2 \\
15.3 \\
16.3 \\
24.6\end{array}$ & $\begin{array}{l}\mathrm{P} \\
\mathrm{P} \\
\mathrm{M} \\
\mathrm{Sr} \\
\mathrm{P}\end{array}$ & $\mathrm{E}$ & $\begin{array}{l}\text { I } \\
\text { I } \\
\text { I } \\
\text { I }\end{array}$ & $\begin{array}{rrr}18 & 29 & 34.72 \\
21 & 30 & 54.71 \\
23 & 10 & 26.69 \\
23 & 57 & 21.15 \\
6 & 48 & \end{array}$ & $\begin{array}{l}+.86 \\
+.71 \\
+.61 \\
+.57\end{array}$ & $\begin{array}{r}+.01 \\
+.03 \\
-.08 \\
-.13\end{array}$ & $\begin{array}{l}+.1 \\
+1.0 \\
-.5 \\
-.9\end{array}$ & $\begin{aligned} & S \\
& S \\
& S \\
& S \\
& S\end{aligned}$ & $\begin{array}{rrr}-26 & 12 & \\
-13 & 55 & 32.9 \\
-2 & 6 & 51.2 \\
+\quad 3 & 51 & 21.4 \\
+25 & 29 & 1.9\end{array}$ & $\begin{array}{l}+4.8 \\
+5.5 \\
+6.2 \\
-.4\end{array}$ & $\begin{array}{l}+1.4 \\
+1.3 \\
+1.9 \\
-.4\end{array}$ & $\begin{array}{l}+1.0 \\
+1.5 \\
+2.4 \\
+.4\end{array}$ \\
\hline Dec. & $\begin{array}{l}27.7 \\
10.2 \\
13.3 \\
14.3 \\
15.3\end{array}$ & $\begin{array}{l}\mathrm{P} \\
\mathrm{Sr} \\
\mathrm{M} \\
\mathrm{Sr} \\
\mathrm{S}\end{array}$ & . & $\begin{array}{l}\text { I }^{\text {II }} \\
\text { I } \\
\text { I }\end{array}$ & $\begin{array}{rrr}9 & 20 & 36.08 \\
21 & 9 & 15.86 \\
23 & 42 & 1.21 \\
0 & 29 & 2.17 \\
1 & 16 & 14.33\end{array}$ & $\begin{array}{l}+.80 \\
+.81 \\
+.65 \\
+.68 \\
+.71\end{array}$ & $\begin{array}{l}+.11 \\
+.06 \\
\pm .03 \\
+.01 \\
+.02\end{array}$ & $\begin{array}{l}+1.0 \\
+.7 \\
+.6 \\
+.7 \\
+1.1\end{array}$ & $\begin{array}{l}\mathbf{S} \\
\mathbf{S} \\
\mathbf{S} \\
\mathbf{S}\end{array}$ & $\begin{array}{r}+144912.3 \\
-1551 \\
+21048.4 \\
+8036.1 \\
+132239.6\end{array}$ & $\begin{array}{r}-1.4 \\
+5.9 \\
+5.0 \\
+5.6\end{array}$ & $\begin{array}{l}+1.5 \\
+2.0 \\
+1.2 \\
+1.9\end{array}$ & $\begin{array}{l}+2.0 \\
+2.0 \\
+\quad .9 \\
+1.5\end{array}$ \\
\hline & $\begin{array}{l}18.4 \\
21.5 \\
22.6 \\
23.6 \\
24.6\end{array}$ & $\begin{array}{l}\mathrm{S} \\
\mathrm{Sr} \\
\mathrm{P} \\
\mathrm{Sr} \\
\mathrm{P}\end{array}$ & & $\begin{array}{ll}\text { I } & \\
& \text { II } \\
& \text { II } \\
& \text { II } \\
\text { II }\end{array}$ & $\begin{array}{rrr}3 & 47 & 1.34 \\
6 & 30 & 33.13 \\
7 & 23 & 54.73 \\
8 & 15 & 18.08 \\
9 & 4 & 36.02\end{array}$ & $\begin{array}{l}+.86 \\
+.89 \\
+.86 \\
+.74 \\
+.74\end{array}$ & $\begin{array}{l}+.11 \\
+.15 \\
+.14 \\
+.04 \\
+.08\end{array}$ & $\begin{array}{r}+1.3 \\
+2.0 \\
+2.0 \\
+\quad .7 \\
+1.0\end{array}$ & $\begin{array}{ll}\mathbf{N} & \\
\mathbf{N} & \mathbf{S} \\
\mathbf{N} & \mathbf{S} \\
& \mathbf{S} \\
& \mathbf{S}\end{array}$ & $\begin{array}{rrr}+24 & 51 & 12.2 \\
+25 & 59 & 5.2 \\
+23 & 47 & 2.7 \\
+20 & 27 & 52.0 \\
+16 & 12 & 37.1\end{array}$ & $\begin{array}{l}+1.9 \\
+.9 \\
-.9 \\
-2.1 \\
-2.2\end{array}$ & $\begin{array}{r}-.9 \\
+.5 \\
-.4 \\
-.8 \\
.0\end{array}$ & $\begin{array}{l}-1.3 \\
+.6 \\
-.1 \\
-.6 \\
+.4\end{array}$ \\
\hline $\begin{array}{l}191 \\
\text { Jan. }\end{array}$ & $\begin{array}{r}6 \\
8.2 \\
14.2 \\
20.5 \\
23.6\end{array}$ & $\begin{array}{l}\mathrm{P} \\
\mathrm{Sr} \\
\mathrm{Sr} \\
\mathrm{Sr}\end{array}$ & & $\begin{array}{ll}\text { I } & \\
\text { I } & \\
& \text { II } \\
& \text { II }\end{array}$ & $\begin{array}{rrr}22 & 31 & 31.21 \\
3 & 29 & 10.57 \\
8 & 48 & 46.55 \\
11 & 10 & 55.59\end{array}$ & $\begin{array}{l}+.81 \\
+.81 \\
+.84 \\
+.61\end{array}$ & $\begin{array}{r}+.04 \\
+.05 \\
+.14 \\
-.02\end{array}$ & $\begin{array}{r}+.4 \\
+\quad .9 \\
+2.1 \\
-\quad .2\end{array}$ & $\begin{array}{l}\mathbf{S} \\
\mathbf{S} \\
\mathbf{S}\end{array}$ & $\begin{array}{rrr}-632 & \\
+24 & 7 & 5.0 \\
+1738 & 49.6 \\
+133 & 41.3\end{array}$ & $\begin{array}{l}+3.6 \\
-2.5 \\
-4.0\end{array}$ & $\begin{array}{l} \pm .9 \\
\pm .5 \\
-.4\end{array}$ & $\begin{array}{r}+.7 \\
+.1 \\
-.4\end{array}$ \\
\hline Feb. & $\begin{array}{r}25.7 \\
7.2 \\
10.2 \\
14.3 \\
15.3\end{array}$ & $\begin{array}{l}\mathrm{Sr} \\
\mathrm{S} \\
\mathrm{S} \\
\mathrm{P} \\
\mathrm{Sr}\end{array}$ & $\underset{\mathrm{W}}{\mathbf{E}}$ & $\begin{array}{l}\text { II } \\
\text { I } \\
\text { I } \\
\text { I }\end{array}$ & $\begin{array}{rrr}12 & 47 & 0.28 \\
0 & 37 & 17.56 \\
3 & 9 & 12.07 \\
6 & 46 & 9.37 \\
7 & 39 & 11.65\end{array}$ & $\begin{array}{l}+.64 \\
+.71 \\
+.84 \\
+1.05 \\
+.87\end{array}$ & $\begin{array}{r}.00 \\
-.09 \\
+.02 \\
+.24 \\
+.07\end{array}$ & $\begin{array}{l}+.1 \\
-1.2 \\
+.5 \\
+3.1 \\
+1.1\end{array}$ & $\begin{array}{l}S \\
N_{N}^{S}\end{array}$ & 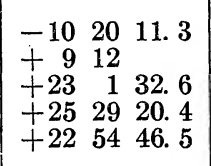 & $\begin{array}{l}-4.3 \\
+3.4 \\
+.6 \\
-2.1\end{array}$ & $\begin{array}{l}-.8 \\
+.5 \\
+.8 \\
-.9\end{array}$ & $\begin{array}{r}-.7 \\
.0 \\
+1.1 \\
-.6\end{array}$ \\
\hline Mar. & \begin{tabular}{r|r}
9.2 \\
11.3 \\
13.3 \\
16.4 \\
17.4
\end{tabular} & $\begin{array}{l}\mathrm{S} \\
\mathrm{P} \\
\mathrm{M} \\
\mathrm{P} \\
\mathrm{Sr}\end{array}$ & & $\begin{array}{l}\text { I } \\
\text { I } \\
\text { I } \\
\text { I } \\
\text { I }\end{array}$ & $\begin{array}{rrr}3 & 40 & 2.43 \\
5 & 29 & 44.65 \\
7 & 17 & 49.09 \\
9 & 48 & 15.43 \\
10 & 36 & 9.97\end{array}$ & $\begin{array}{l}+.92 \\
+1.04 \\
+1.01 \\
+.85 \\
+.84\end{array}$ & $\begin{array}{l}+.04 \\
+.15 \\
+.14 \\
+.05 \\
+.07\end{array}$ & $\begin{array}{r}+.5 \\
+2.1 \\
+2.0 \\
+.0 \\
+.6\end{array}$ & $\underbrace{}_{N}{ }_{N}^{S}$ & $\begin{array}{rrr}+24 & 31 & 4.1 \\
+26 & 51 & 59.7 \\
+23 & 59 & 10.0 \\
+11 & 34 & 1.9 \\
+ & 56 & 47.4\end{array}$ & $\begin{array}{l}+2.0 \\
+2.2 \\
-1.8 \\
-2.3 \\
-4.0\end{array}$ & $\begin{array}{l}-.6 \\
+1.4 \\
+.6 \\
+1.8 \\
+.7\end{array}$ & $\begin{array}{l}-1.0 \\
+1.1 \\
-.3 \\
+2.1 \\
+1.1\end{array}$ \\
\hline Apr. & $\begin{array}{l}22.6 \\
23.7 \\
24.7 \\
25.7 \\
10.3\end{array}$ & $\begin{array}{l}\mathrm{P} \\
\mathrm{Sr} \\
\mathrm{M} \\
\mathrm{P} \\
\mathrm{M}\end{array}$ & & $\mathrm{I}_{\text {I }} \begin{array}{l}\text { II } \\
\text { II } \\
\text { II } \\
\text { II }\end{array}$ & $\begin{array}{rrrr}14 & 57 & 52.95 \\
16 & 0 & 17.26 \\
17 & 5 & 30.33 \\
18 & 11 & 28.05 \\
7 & 47 & 56.49\end{array}$ & $\begin{array}{l}+.83 \\
+.69 \\
+.78 \\
+.66 \\
+.92\end{array}$ & $\begin{array}{l}+.07 \\
\pm .05 \\
+.07 \\
+.01 \\
+.04\end{array}$ & $\begin{array}{l}+.8 \\
+.7 \\
+1.0 \\
+.2\end{array}$ & $\mathrm{~N}_{\mathrm{N}}^{\stackrel{\mathrm{S}}{\mathrm{S}}}$ & $\begin{array}{rrr}-22 & 6 & \\
-25 & 14 & 53.7 \\
-26 & 41 & 44.5 \\
-26 & 15 & 25.0 \\
+22 & 1 & 34.0\end{array}$ & $\begin{array}{l}-1.8 \\
-1.4 \\
-.1 \\
-2.9\end{array}$ & $\begin{array}{l}-.7 \\
-1.4 \\
-1.0 \\
-.9\end{array}$ & $\begin{array}{l}-.8 \\
-1.3 \\
-1.0 \\
-.8\end{array}$ \\
\hline & $\begin{array}{l}12.3 \\
13.3 \\
15.4 \\
17.5 \\
18.5\end{array}$ & $\begin{array}{l}\mathrm{S} \\
\mathrm{M} \\
\mathrm{S} \\
\mathrm{M} \\
\mathrm{P}\end{array}$ & W & $\begin{array}{ll}\text { I } & \\
\text { I } & \\
\text { I } & \\
\text { I } & \text { II } \\
& \text { II }\end{array}$ & 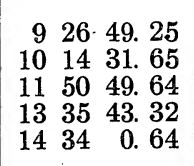 & $\begin{array}{l}+.86 \\
+.84 \\
+.80 \\
+.75 \\
+.86\end{array}$ & $\begin{array}{r}+.01 \\
+.02 \\
.00 \\
-.06 \\
+.03\end{array}$ & $\begin{array}{l}+.1 \\
-.1 \\
-.8 \\
-1.3 \\
+.6\end{array}$ & $\begin{array}{l}\mathrm{N} \\
\stackrel{N}{N} \\
\stackrel{S}{\mathbf{S}}\end{array}$ & 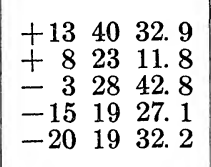 & $\begin{array}{l}-3.8 \\
-4.4 \\
-3.9 \\
-3.8 \\
-4.2\end{array}$ & $\begin{array}{l}+.4 \\
+.5 \\
+1.6 \\
+.6 \\
+.9\end{array}$ & $\begin{array}{l}+.5 \\
+.6 \\
+1.6 \\
+.3 \\
+.6\end{array}$ \\
\hline
\end{tabular}




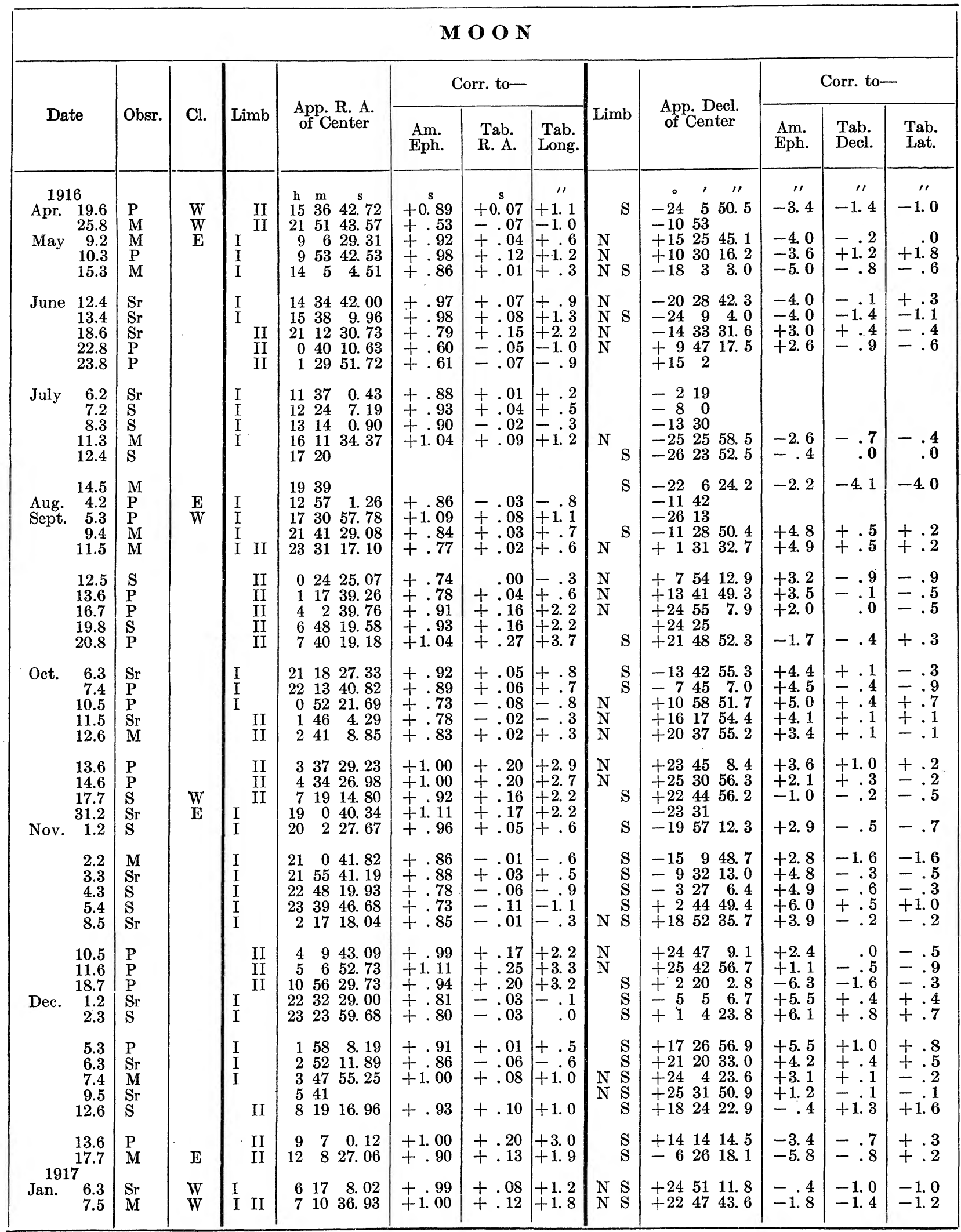




\begin{tabular}{|c|c|c|c|c|c|c|c|c|c|c|c|c|c|}
\hline \multicolumn{14}{|c|}{$\mathbf{M O O N}$} \\
\hline \multirow{2}{*}{\multicolumn{2}{|c|}{ Date }} & \multirow[b]{2}{*}{ Obsr. } & \multirow[b]{2}{*}{$\mathrm{Cl}$. } & \multirow[b]{2}{*}{ Limb } & \multirow{2}{*}{$\begin{array}{l}\text { App. R. A. } \\
\text { of Center }\end{array}$} & \multicolumn{3}{|c|}{ Corr. to- } & \multirow[b]{2}{*}{$\operatorname{Limb}$} & \multirow{2}{*}{$\begin{array}{l}\text { App. Decl. } \\
\text { of Center }\end{array}$} & \multicolumn{3}{|c|}{ Corr. to- } \\
\hline & & & & & & $\begin{array}{l}\text { Am. } \\
\text { Eph. }\end{array}$ & $\begin{array}{l}\text { Tab. } \\
\text { R. A. }\end{array}$ & $\begin{array}{l}\text { Tab. } \\
\text { Long. }\end{array}$ & & & $\begin{array}{l}\text { Am. } \\
\text { Eph. }\end{array}$ & $\begin{array}{l}\text { Tab. } \\
\text { Decl. }\end{array}$ & $\begin{array}{l}\text { Tab. } \\
\text { Lat. }\end{array}$ \\
\hline $\begin{array}{l}1917 \\
\text { Jan. } \\
\end{array}$ & $\begin{array}{r}7.5 \\
9.6 \\
18.8 \\
26.2 \\
30.3\end{array}$ & $\begin{array}{l}\mathrm{S} \\
\mathrm{P} \\
\mathrm{P} \\
\mathrm{Sr} \\
\mathrm{Sr}\end{array}$ & W & $\begin{array}{ll} & \text { II } \\
& \text { II } \\
\text { II } \\
\text { I }\end{array}$ & $\begin{array}{rrc}\mathrm{h} & \mathrm{m} & \mathrm{s} \\
8 & 1 & 41.39 \\
8 & 50 & 16.75 \\
16 & 18 & 43.66 \\
23 & 36 & 32.60 \\
3 & 12 & 17.67\end{array}$ & $\begin{array}{r}\mathrm{s} \\
+0.89 \\
+\quad .93 \\
+1.09 \\
+\quad .80 \\
+\quad .96\end{array}$ & $\begin{array}{r}\mathrm{s} \\
+0.05 \\
+\quad .11 \\
+.19 \\
+.00 \\
+.08\end{array}$ & $\begin{array}{r}1 \prime \\
+0.2 \\
+1.1 \\
+2.5 \\
+.0 \\
+\quad .9\end{array}$ & $\begin{array}{l}\mathbf{S} \\
\mathbf{S}\end{array}$ & $\begin{array}{rcc}\circ & \prime \prime \\
+19 & 41 & 45.5 \\
+15 & 45 & 32.3 \\
+25 & 4 & \\
+2 & 42 & \\
+22 & 31 & 39.4\end{array}$ & $\begin{array}{r}\prime \prime \\
+0.6 \\
-.9 \\
+2.5\end{array}$ & $\begin{array}{r}1 \prime \\
+1.9 \\
+1.3 \\
-.4\end{array}$ & $\begin{array}{r}1 \prime \\
+2.1 \\
+1.8 \\
-.8\end{array}$ \\
\hline $\begin{array}{l}\text { Feb. } \\
\text { Mar. }\end{array}$ & $\begin{array}{r}1.3 \\
6.5 \\
7.5 \\
16.7 \\
6.4\end{array}$ & $\begin{array}{l}\mathrm{M} \\
\mathrm{P} \\
\mathrm{P} \\
\mathrm{Sr} \\
\mathrm{P}\end{array}$ & & $\begin{array}{ll}\text { I } & \\
\text { I } & \text { II } \\
& \text { II } \\
& \text { II } \\
\text { I } & \end{array}$ & \begin{tabular}{rrr|}
5 & 4 & 7.40 \\
9 & 21 & 29.78 \\
10 & 7 & 3.92 \\
17 & 59 & 51.17 \\
9 & 51 & 43.96
\end{tabular} & $\begin{array}{r}+.98 \\
+.90 \\
+\quad .88 \\
+1.06 \\
+\quad .90\end{array}$ & $\begin{array}{r}+.08 \\
+.11 \\
+.11 \\
+.17 \\
+.11\end{array}$ & $\begin{array}{l}+1.2 \\
+1.7 \\
+1.7 \\
+2.3 \\
+1.7\end{array}$ & $\begin{array}{l}\mathbf{S} \\
\mathbf{S} \\
\mathbf{S}\end{array}$ & $\begin{array}{rrr}+25 & 40 & 2.5 \\
+124752.6 \\
+755 & 1.3 \\
+2510 & \\
+936 & 25.1\end{array}$ & $\begin{array}{l}+3.0 \\
-3.2 \\
-4.1 \\
-4.4\end{array}$ & $\begin{array}{l} \pm 1.6 \\
-.4 \\
-.7 \\
-.8\end{array}$ & $\begin{array}{r}+1.5 \\
+.2 \\
.0 \\
-.1\end{array}$ \\
\hline & $\begin{array}{r}9.5 \\
15.8 \\
17.8 \\
28.2 \\
29.2\end{array}$ & $\begin{array}{l}\mathrm{M} \\
\mathrm{M} \\
\mathrm{P} \\
\mathrm{S} \\
\mathrm{M}\end{array}$ & & $\begin{array}{ll} & \text { II } \\
& \text { II } \\
\text { II } & \\
\text { I } & \end{array}$ & $\begin{array}{rrr}12 & 7 & 23.44 \\
17 & 35 & 59.84 \\
19 & 42 & 5.95 \\
5 & 18 & 7.19 \\
6 & 14 & 25.13\end{array}$ & $\begin{array}{l}+.85 \\
+.85 \\
+.89 \\
+1.00 \\
+1.07\end{array}$ & $\begin{array}{l}+.12 \\
+.06 \\
+.11 \\
+.07 \\
+.16\end{array}$ & $\begin{array}{r}+1.8 \\
+.8 \\
+1.5 \\
+1.0 \\
+2.1\end{array}$ & $\mathrm{~N}$ & $\begin{array}{rrr}-6 & 9 & 32.6 \\
-25 & 18 & \\
-20 & 47 & \\
+25 & 17 & \\
+24 & 31 & 14.6\end{array}$ & -5.1 & -.7 & +.2 \\
\hline Apr. & $\begin{array}{r}30.3 \\
3.3 \\
7.5 \\
9.6 \\
10.6\end{array}$ & $\begin{array}{l}\mathrm{Sr} \\
\mathrm{P} \\
\mathrm{Sr} \\
\mathrm{Sr} \\
\mathrm{S}\end{array}$ & & $\begin{array}{ll}\text { I } & \\
\text { I } & \\
& \text { II }\end{array}$ & $\begin{array}{rrr}7 & 8 & 16.14 \\
10 & 20 & 7.17 \\
13 & 27 & 12.27 \\
15 & 16 & \\
16 & 15 & 18.89\end{array}$ & $\begin{array}{l}+1.02 \\
+.89 \\
+.80 \\
+.80\end{array}$ & $\begin{array}{l}+.13 \\
+.08 \\
+.04 \\
+.06\end{array}$ & $\begin{array}{l}+1.8 \\
+.7 \\
+.5 \\
+.7\end{array}$ & $\begin{array}{l}\mathrm{N} \\
\mathrm{N} \\
\mathbf{S} \\
\mathrm{S} \\
\mathrm{S}\end{array}$ & $\begin{array}{rrr}+22 & 32 & 32.5 \\
+6 & 19 & 16.0 \\
-14 & 26 & 29.1 \\
-22 & 13 & 3.2 \\
-24 & 25 & 29.7\end{array}$ & $\begin{array}{l}-1.8 \\
-3.6 \\
-4.5 \\
-3.2 \\
-.7\end{array}$ & $\begin{array}{l}-.9 \\
\pm .9 \\
\pm .1 \\
\bar{t} .6 \\
+.7\end{array}$ & $\begin{array}{l}-.6 \\
+1.4 \\
\pm .2 \\
+.6 \\
+.9\end{array}$ \\
\hline May & \begin{tabular}{r|}
13.7 \\
14.7 \\
30.3 \\
1.3 \\
2.4
\end{tabular} & $\begin{array}{l}\mathbf{S} \\
\mathbf{P} \\
\mathbf{M} \\
\mathbf{P} \\
\mathbf{S r}\end{array}$ & $\begin{array}{l}\mathrm{W} \\
\mathrm{E}\end{array}$ & $\begin{array}{ll} & \text { II } \\
\text { I } & \text { II } \\
\text { I } & \\
\text { I } & \end{array}$ & $\begin{array}{rrr}19 & 21 & 51.13 \\
20 & 21 & 46.91 \\
10 & 1 & 46.97 \\
10 & 46 & 43.99 \\
11 & 31 & 52.33\end{array}$ & $\begin{array}{r}+.70 \\
+.65 \\
+.98 \\
+1.09 \\
+.98\end{array}$ & $\begin{array}{l}+.01 \\
.02 \\
+.13 \\
+.26 \\
+.15\end{array}$ & \begin{tabular}{l}
+.3 \\
\hdashline .3 \\
+1.6 \\
+3.1 \\
+1.7
\end{tabular} & $\begin{array}{l}\mathrm{N} \\
\mathrm{N} \\
\mathrm{N} \\
\mathrm{N}\end{array}$ & $\begin{array}{rrr}-21 & 41 & 58.6 \\
-17 & 45 & \\
+8 & 7 & 43.6 \\
+3 & 3 & 14.4 \\
-2 & 12 & 23.5\end{array}$ & $\begin{array}{l}+2.7 \\
-4.2 \\
-4.2 \\
-4.9\end{array}$ & $\begin{array}{l}+1.3 \\
+.3 \\
+.9 \\
+.7\end{array}$ & $\begin{array}{l}+1.2 \\
+1.0 \\
+2.4 \\
+1.6\end{array}$ \\
\hline June & $\begin{array}{r}3.4 \\
11.7 \\
14.8 \\
25.2 \\
4.4\end{array}$ & $\begin{array}{l}\mathrm{Sr} \\
\mathrm{P} \\
\mathrm{Sr} \\
\mathrm{P} \\
\mathrm{P}\end{array}$ & & $\begin{array}{ll}\text { I } & \\
& \text { II } \\
\text { I } & \\
\text { I } & \end{array}$ & $\begin{array}{rrr}12 & 18 & 14.08 \\
20 & 3 & 58.24 \\
22 & 51 & 1.24 \\
8 & 7 & 59.40 \\
16 & 29 & 4.39\end{array}$ & $\begin{array}{l}+.97 \\
+.63 \\
+.52 \\
+1.02 \\
+.88\end{array}$ & $\begin{array}{l}+.14 \\
\pm .01 \\
=.08 \\
+.17 \\
.00\end{array}$ & $\begin{array}{l}+1.7 \\
-0.3 \\
-1.1 \\
+2.4 \\
-.2\end{array}$ & $\begin{array}{l}\mathrm{N} \\
\mathrm{N}\end{array}$ & $\begin{array}{rrr}-728 & 40.8 \\
-18 & 50 & 0.9 \\
-2 & 27 & \\
+18 & & \\
-24 & 34 & 16.7\end{array}$ & $\begin{array}{l}-5.3 \\
+1.5\end{array}$ & \pm .3 & $\begin{array}{l}+1.2 \\
-.8\end{array}$ \\
\hline 1918 & $\begin{array}{r}7.6 \\
13.7 \\
8\end{array}$ & $\stackrel{\mathrm{M}}{\mathrm{P}}$ & $\mathrm{E}$ & $\begin{array}{l}\text { II } \\
\text { II }\end{array}$ & $\begin{array}{rrr}19 & 42 & 36.54 \\
1 & 13 & 52.87\end{array}$ & $\begin{array}{r}+78 \\
+\quad .70\end{array}$ & $\begin{array}{r}+.05 \\
+.08\end{array}$ & $\begin{array}{r}. \\
+.5 \\
+1.0\end{array}$ & $\mathrm{~N}$ & $\begin{array}{l}-20828.1 \\
+1323\end{array}$ & +.4 & -1.6 & -1.8 \\
\hline Jan. & $\begin{array}{l}19.2 \\
21.3\end{array}$ & $\begin{array}{l}\mathrm{Sr} \\
\mathrm{Sr}\end{array}$ & $W$ & I & $\begin{array}{lll}1 & 59 & 35.99 \\
3 & 56 & 19.14\end{array}$ & $\begin{array}{r}+.89 \\
+\quad .92\end{array}$ & $=.01$ & \pm .1 & $\begin{array}{l}\mathbf{S} \\
\mathbf{S}\end{array}$ & $\begin{array}{r}+165430.4 \\
+23 \quad 254.7\end{array}$ & $\begin{array}{l}+5.0 \\
+1.5\end{array}$ & $\begin{array}{l}+.9 \\
-.9\end{array}$ & $\begin{array}{l}+.8 \\
-.9\end{array}$ \\
\hline Feb. & $\begin{array}{l}22.3 \\
23.3 \\
25.5 \\
15.2 \\
18.3\end{array}$ & $\begin{array}{l}\mathrm{P} \\
\mathrm{M} \\
\mathrm{Sr} \\
\mathrm{Sr} \\
\mathrm{M}\end{array}$ & & $\begin{array}{l}\text { I } \\
\text { I } \\
\text { I } \\
\text { I } \\
\text { I }\end{array}$ & $\begin{array}{lll}4 & 55 & 44.34 \\
5 & 54 & 24.33 \\
7 & 45 & 11.60 \\
1 & 39 & 36.08 \\
4 & 37 & 52.04\end{array}$ & $\begin{array}{r}+1.05 \\
+.94 \\
+.80 \\
+.74 \\
+.90\end{array}$ & $\begin{array}{r}+.11 \\
\pm .03 \\
\pm .05 \\
-.12 \\
.00\end{array}$ & $\begin{array}{r}+1.5 \\
+.3 \\
-.7 \\
-1.6 \\
.0\end{array}$ & $\begin{array}{r}\mathbf{S} \\
\mathbf{S} \\
\mathbf{S} \\
\mathrm{S}\end{array}$ & 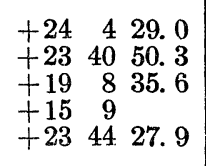 & $\begin{array}{l}+1.9 \\
+.1 \\
-1.3 \\
+2.0\end{array}$ & $\begin{array}{l} \pm .4 \\
\pm .4 \\
-.1 \\
+.6\end{array}$ & $\begin{array}{l} \pm .2 \\
\pm .4 \\
-.2 \\
+.5\end{array}$ \\
\hline Mar. & $\begin{array}{r}20.3 \\
21.4 \\
23.4 \\
26.5 \\
1.6\end{array}$ & $\begin{array}{l}\mathrm{M} \\
\mathrm{Sr} \\
\mathrm{M} \\
\mathrm{Sr} \\
\mathrm{Sr}\end{array}$ & & $\begin{array}{ll}\text { I } & \\
\text { I } & \\
\text { I } & \\
& \text { II } \\
& \text { II }\end{array}$ & $\begin{array}{rrr}6 & 33 & 43.98 \\
7 & 28 & 4.48 \\
9 & 8 & 25.29 \\
11 & 25 & 4.60 \\
13 & 42 & 50.13\end{array}$ & $\begin{array}{r}+.93 \\
+.92 \\
+.80 \\
+.78 \\
+.68\end{array}$ & $\begin{array}{l}+.05 \\
+.07 \\
+.01 \\
+.06 \\
+.02\end{array}$ & $\begin{array}{l}+.8 \\
+1.2 \\
+.6 \\
+1.0 \\
+.3\end{array}$ & $\begin{array}{ll}N & S \\
N & \\
N & S \\
& S \\
& S\end{array}$ & $\begin{array}{rrr}+22 & 30 & 21.3 \\
+20 & 4 & 3.4 \\
+12 & 37 & 52.4 \\
-1 & 39 & 44.3 \\
-15 & 16 & 28.8\end{array}$ & $\begin{array}{l}-.7 \\
-2.4 \\
-4.3 \\
-4.2 \\
-4.9\end{array}$ & $\begin{array}{l}-.4 \\
-1.2 \\
-1.7 \\
=.4 \\
-1.4\end{array}$ & $\begin{array}{l}-.3 \\
-1.0 \\
-1.5 \\
+.1 \\
-1.2\end{array}$ \\
\hline & $\begin{array}{r}2.6 \\
3.7 \\
15.2 \\
16.2 \\
18.2\end{array}$ & $\begin{array}{l}\mathrm{P} \\
\mathrm{M} \\
\mathrm{P} \\
\mathrm{M} \\
\mathrm{Sr}\end{array}$ & & $\begin{array}{ll}\text { II } \\
\text { II } \\
\text { I } \\
\text { I }\end{array}$ & $\begin{array}{rrr}14 & 32 & 30.09 \\
15 & 24 & 56.77 \\
2 & 12 & 27.30 \\
3 & 14 & 3.70 \\
5 & 16 & 44.84\end{array}$ & $\begin{array}{l}+.73 \\
+.76 \\
+.96 \\
+.95 \\
+.99\end{array}$ & $\begin{array}{l}+.02 \\
+.04 \\
+.05 \\
+.03 \\
+.08\end{array}$ & $\begin{array}{r}+.3 \\
+.6 \\
+.7 \\
+1.1\end{array}$ & $\begin{array}{l}S \\
S\end{array}$ & $\begin{array}{rrr}-18 & 50 & 13.8 \\
-21 & 35 & 2.0 \\
+17 & 25 & \\
+21 & 0 & 14.2 \\
+23 & 40 & 8.4\end{array}$ & $\begin{array}{l}-3.3 \\
-3.1 \\
+2.1 \\
+1.3\end{array}$ & $\begin{array}{l}-.1 \\
-.6 \\
-.8 \\
+.5\end{array}$ & $\begin{array}{l}+.1 \\
-.3 \\
-1.0 \\
+.4\end{array}$ \\
\hline & $\begin{array}{l}22.4 \\
23.4 \\
25.4 \\
26.5 \\
27.5\end{array}$ & $\begin{array}{l}\mathrm{P} \\
\mathrm{Sr} \\
\mathrm{Sr} \\
\mathrm{P} \\
\mathrm{P}\end{array}$ & W & $\begin{array}{ll}\text { I } & \\
I^{I} & \\
& \text { II }\end{array}$ & $\begin{array}{rrr}8 & 53 & \\
9 & 40 & 12.37 \\
11 & 10 & 26.01 \\
11 & 55 & 7.79 \\
12 & 40 & 35.64\end{array}$ & $\begin{array}{r}+.80 \\
+.76 \\
+.84 \\
+.78\end{array}$ & $\begin{array}{l}+.03 \\
+.03 \\
+.13 \\
+.09\end{array}$ & $\begin{array}{r}+3 \\
+\quad .3 \\
+\quad .3 \\
+1.8 \\
+1.5\end{array}$ & $\begin{array}{ll}\mathbf{N} & \\
\mathbf{N} & \\
\mathbf{N} & \mathbf{S} \\
& \mathbf{S} \\
& \mathrm{S}\end{array}$ & 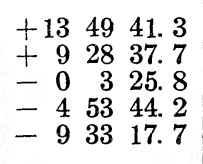 & $\begin{array}{l}-2.9 \\
-2.9 \\
-3.7 \\
-4.4 \\
-4.9\end{array}$ & $\begin{array}{l}-.2 \\
+.4 \\
\pm .5 \\
\pm .2 \\
-.7\end{array}$ & $\begin{array}{r}-.2 \\
+\quad .6 \\
+.7 \\
+.8 \\
.0\end{array}$ \\
\hline
\end{tabular}




\begin{tabular}{|c|c|c|c|c|c|c|c|c|c|c|c|c|c|}
\hline \multicolumn{14}{|c|}{$\mathbf{M O O N}$} \\
\hline \multirow{2}{*}{\multicolumn{2}{|c|}{ Date }} & \multirow[b]{2}{*}{ Obsr. } & \multirow[b]{2}{*}{ Cl. } & \multirow[b]{2}{*}{ Limb } & \multirow{2}{*}{$\begin{array}{l}\text { App. R. A. } \\
\text { of Center }\end{array}$} & \multicolumn{3}{|c|}{ Corr. to- } & \multirow[b]{2}{*}{ Limb } & \multirow{2}{*}{$\begin{array}{l}\text { App. Decl. } \\
\text { of Center }\end{array}$} & \multicolumn{3}{|c|}{ Corr. to- } \\
\hline & & & & & & $\begin{array}{l}\text { Am. } \\
\text { Eph. }\end{array}$ & $\begin{array}{l}\text { Tab. } \\
\text { R. A. }\end{array}$ & $\begin{array}{l}\text { Tab. } \\
\text { Long. }\end{array}$ & & & Am. & $\begin{array}{l}\text { Tab. } \\
\text { Decl. }\end{array}$ & $\begin{array}{l}\text { Tab. } \\
\text { Lat. }\end{array}$ \\
\hline $\begin{array}{l}1918 \\
\text { Mar. } \\
\text { Apr. }\end{array}$ & $\begin{array}{r}18 \\
28.5 \\
29.6 \\
30.6 \\
4.8 \\
5.8\end{array}$ & $\begin{array}{l}\mathrm{M} \\
\mathrm{Sr} \\
\mathrm{P} \\
\mathrm{Sr} \\
\mathrm{M}\end{array}$ & $\mathrm{W}$ & $\begin{array}{l}\text { II } \\
\text { II } \\
\text { II } \\
\text { II } \\
\text { II }\end{array}$ & \begin{tabular}{rrr}
$\mathrm{h}$ & $\mathrm{m}$ & \multicolumn{1}{c}{$\mathrm{s}$} \\
13 & 27 & 32.60 \\
14 & 16 & 34.53 \\
15 & 8 & 5.03 \\
19 & 52 & 43.87 \\
20 & 50 & 15.68
\end{tabular} & $\begin{array}{l}\mathrm{s} \\
+0.71 \\
+.66 \\
+.84 \\
+.90 \\
+.81\end{array}$ & $\begin{array}{r}s \\
+0.04 \\
.00 \\
+.18 \\
+.18 \\
+.09\end{array}$ & $\begin{array}{r} \\
+2 \\
+0.7 \\
+2.3 \\
+2.5 \\
+1.2\end{array}$ & $\begin{array}{l}\mathbf{S} \\
\mathbf{S} \\
\mathbf{S}\end{array}$ & $\begin{array}{rcc}\circ & \prime & \prime \prime \\
-13 & 51 & 19.4 \\
-17 & 36 & 13.4 \\
-20 & 35 & 46.1 \\
-18 & 13 & \\
-13 & 55 & \end{array}$ & $\begin{array}{r}\prime \prime \\
-4.6 \\
-2.7 \\
-3.8\end{array}$ & $\begin{array}{r}11 \\
-0.7 \\
+\quad .8 \\
-1.0\end{array}$ & $\begin{array}{r}1 \prime \\
-0.3 \\
+\quad .8 \\
-.2\end{array}$ \\
\hline May & $\begin{array}{r}15.2 \\
18.3 \\
21.3 \\
27.6 \\
1.7\end{array}$ & $\begin{array}{l}\mathrm{M} \\
\mathbf{M} \\
\mathrm{M} \\
\mathrm{P} \\
\mathrm{P}\end{array}$ & & $\begin{array}{ll}\text { I } & \\
\text { I } & \\
\text { I } & \\
& \text { II } \\
& \text { II }\end{array}$ & $\begin{array}{rrr}5 & 51 & 34.38 \\
8 & 35 & 59.00 \\
10 & 55 & 14.81 \\
15 & 45 & 3.19 \\
19 & 33 & 45.70\end{array}$ & $\begin{array}{r}+1.00 \\
+.88 \\
+.85 \\
+.72 \\
+.76\end{array}$ & $\begin{array}{l}+.05 \\
+.03 \\
+.08 \\
+.03 \\
+.13\end{array}$ & $\begin{array}{r}+.6 \\
+.7 \\
+1.7 \\
+.6 \\
+1.6\end{array}$ & $\begin{array}{l}\mathrm{N} \\
\mathrm{N} \\
\mathrm{N}\end{array}$ & $\begin{array}{rrr}+23 & 4 \\
+14 & 59 & 35.9 \\
+1 & 27 & 0.4 \\
-21 & 58 & 45.8 \\
-19 & 8 & 28.1\end{array}$ & $\begin{array}{l}-3.5 \\
-5.8 \\
-3.8 \\
+1.1\end{array}$ & $\begin{array}{l}-.8 \\
-1.4 \\
-1.2 \\
-.7\end{array}$ & $\begin{array}{l}-.6 \\
-.7 \\
-1.0 \\
-1.0\end{array}$ \\
\hline & $\begin{array}{l}16.2 \\
17.2 \\
18.3 \\
20.3 \\
22.4\end{array}$ & $\begin{array}{l}\mathrm{Sr} \\
\mathrm{P} \\
\mathrm{Sr} \\
\mathrm{P} \\
\mathrm{Sr}\end{array}$ & & $\begin{array}{l}\text { I } \\
\text { I } \\
\text { I } \\
\text { I }\end{array}$ & $\begin{array}{rrr}9 & 5 & \\
9 & 52 & 50.03 \\
10 & 38 & 27.45 \\
12 & 8 & 10.09 \\
13 & 42 & 2.60\end{array}$ & $\begin{array}{l}+.86 \\
+.90 \\
+.82 \\
+.84\end{array}$ & $\begin{array}{r}+.04 \\
+.07 \\
+.02 \\
+.04\end{array}$ & $\begin{array}{r}.0 \\
+1.1 \\
+.1 \\
+.6\end{array}$ & $\begin{array}{l}\mathbf{N} \\
\mathbf{N} \\
\mathbf{N} \\
\mathbf{N} \\
\mathbf{N}\end{array}$ & $\begin{array}{r}+121941.8 \\
+74936.6 \\
+3516.4 \\
-62748.5 \\
-15 \quad 452.0\end{array}$ & $\begin{array}{l}-4.5 \\
-5.5 \\
-4.7 \\
-4.5 \\
-4.8\end{array}$ & $\begin{array}{r}-1.3 \\
-1.4 \\
-.2 \\
+.6 \\
.0\end{array}$ & $\begin{array}{l}-1.2 \\
-1.4 \\
+.4 \\
+.9 \\
+.3\end{array}$ \\
\hline June & $\begin{array}{r}23.4 \\
25.5 \\
26.5 \\
31.6 \\
3.7\end{array}$ & $\begin{array}{l}\mathrm{Sr} \\
\mathrm{Sr} \\
\mathrm{Sr} \\
\mathrm{P} \\
\mathrm{Sr}\end{array}$ & & $\begin{array}{ll}\text { I } & \\
& \text { II } \\
& \text { II } \\
& \text { II } \\
& \text { II }\end{array}$ & $\begin{array}{rrr}14 & 32 & 26.55 \\
16 & 21 & 33.47 \\
17 & 19 & 23.11 \\
22 & 2 & 57.00 \\
0 & 46 & 27.72\end{array}$ & $\begin{array}{r}+.76 \\
+.86 \\
+.90 \\
+.41 \\
+.45\end{array}$ & $\begin{array}{r}-.03 \\
+.06 \\
+.13 \\
-.19 \\
-.17\end{array}$ & $\begin{array}{l}-.1 \\
+.7 \\
+1.7 \\
-3.4 \\
-2.3\end{array}$ & $\begin{array}{ll}\mathbf{N} & \mathbf{S} \\
\mathbf{N} & \mathrm{S} \\
\mathbf{N} & \mathrm{S} \\
\mathrm{N} & \end{array}$ & $\begin{array}{rrr}-18 & 35 & 11.4 \\
-22 & 53 & 6.4 \\
-23 & 15 & 44.6 \\
-\quad 6 & 45 & 5.2 \\
+10 & 17 & \end{array}$ & $\begin{array}{l}-5.1 \\
-1.4 \\
-.7 \\
+2.2\end{array}$ & $\begin{array}{r}-.9 \\
+.9 \\
+.4 \\
-1.7\end{array}$ & $\begin{array}{l}-.9 \\
+1.1 \\
+.5 \\
-.7\end{array}$ \\
\hline & $\begin{array}{l}15.2 \\
17.3 \\
19.3 \\
23.5 \\
26.6\end{array}$ & $\begin{array}{l}\mathrm{P} \\
\mathrm{M} \\
\mathrm{M} \\
\mathrm{Sr} \\
\mathrm{P}\end{array}$ & & $\begin{array}{ll}\text { I } & \\
\text { I } & \\
\text { I } & \\
\text { I } & \\
& \text { II }\end{array}$ & $\begin{array}{rrr}11 & 5 & 13.70 \\
12 & 35 & 37.33 \\
14 & 11 & 40.45 \\
17 & 55 & 29.06 \\
20 & 50 & 50.78\end{array}$ & $\begin{array}{l}+.82 \\
+.92 \\
+.84 \\
+1.04 \\
+.62\end{array}$ & $\begin{array}{l}-.01 \\
+.10 \\
+.01 \\
+.21 \\
+.10\end{array}$ & $\begin{array}{r}-.8 \\
+1.5 \\
+2.0 \\
+1.5\end{array}$ & $\begin{array}{l}\mathrm{N} \\
\mathrm{N} \\
\mathrm{N} \\
\mathrm{N} \\
\mathrm{N}\end{array}$ S & 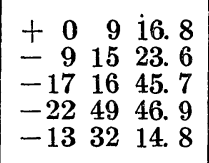 & $\begin{array}{l}-2.5 \\
-5.3 \\
-4.0 \\
-.3 \\
+2.8\end{array}$ & $\begin{array}{r}+2.1 \\
+.3 \\
+.6 \\
-.0 \\
-.4\end{array}$ & $\begin{array}{l}+2.0 \\
+.5 \\
+.7 \\
+.1 \\
+.1\end{array}$ \\
\hline $\begin{array}{l}\text { July } \\
\text { Oct. }\end{array}$ & $\begin{array}{r}27.6 \\
1.8 \\
10.2 \\
14.3 \\
15.3\end{array}$ & $\begin{array}{l}\mathrm{Sr} \\
\mathrm{Sr} \\
\mathrm{P} \\
\mathrm{P} \\
\mathrm{P}\end{array}$ & & $\begin{array}{ll} & \text { II } \\
\text { I } & \text { II } \\
\text { I } & \\
\text { I } & \end{array}$ & $\begin{array}{rrr}21 & 46 & 17.64 \\
1 & 25 & 11.10 \\
17 & 15 & 23.20 \\
20 & 58 & 31.94 \\
21 & 54 & 5.95\end{array}$ & $\begin{array}{r}+.43 \\
+.54 \\
+.93 \\
+.85 \\
+.90\end{array}$ & $\begin{array}{l}-.26 \\
\bar{z} .12 \\
\pm .01 \\
\pm .03 \\
+.06\end{array}$ & $\begin{array}{r}-3.9 \\
-2.5 \\
+.1 \\
+.8 \\
+.5\end{array}$ & $\begin{array}{ll}\mathbf{N} & \\
\mathbf{N} & \\
& \mathbf{S} \\
\mathbf{S}\end{array}$ & $\begin{array}{r}82557.0 \\
-134333.6 \\
-2236 \\
-123512.2 \\
-73234.0\end{array}$ & $\begin{array}{l}+3.1 \\
+1.8 \\
+4.0 \\
+4.7\end{array}$ & $\begin{array}{l}-.8 \\
-2.0 \\
-.8 \\
-.8\end{array}$ & $\begin{array}{l}+.4 \\
-1.3 \\
-.7 \\
-1.2\end{array}$ \\
\hline & $\begin{array}{l}17.4 \\
18.5 \\
21.6 \\
22.6 \\
28.8\end{array}$ & $\begin{array}{l}\mathrm{Sr} \\
\mathrm{Sr} \\
\mathrm{M} \\
\mathrm{M} \\
\mathrm{P}\end{array}$ & & $\begin{array}{ll}\text { I } & \\
& \\
& \text { II } \\
& \text { II } \\
& \text { II }\end{array}$ & $\begin{array}{rrr}23 & 47 & 24.39 \\
0 & 47 & \\
3 & 57 & 50.93 \\
5 & 3 & 1.08 \\
10 & 28 & 19.66\end{array}$ & $\begin{array}{r}+.84 \\
+.90 \\
+.82 \\
+.81\end{array}$ & $\begin{array}{l}+.07 \\
+.19 \\
+.14 \\
+.15\end{array}$ & $\begin{array}{l}+.9 \\
+2.8 \\
+1.8 \\
+2.1\end{array}$ & $\begin{array}{ll} & \text { S } \\
N & \stackrel{S}{N} \\
N & \end{array}$ & $\begin{array}{l}+4210.0 \\
+94833.7 \\
+213522.5 \\
+223155.2 \\
+44\end{array}$ & $\begin{array}{l}+5.9 \\
+5.6 \\
+3.8 \\
+1.6\end{array}$ & $\begin{array}{l}+.3 \\
+.6 \\
+1.7 \\
+.5\end{array}$ & $\begin{array}{l}-.2 \\
+.6 \\
+1.1 \\
+.2\end{array}$ \\
\hline Nov. & \begin{tabular}{r|}
8.2 \\
11.2 \\
12.3 \\
14.3 \\
15.4
\end{tabular} & $\begin{array}{l}\mathrm{Sr} \\
\mathrm{M} \\
\mathrm{Sr} \\
\mathrm{M} \\
\mathrm{Sr}\end{array}$ & & $\begin{array}{l}\text { I } \\
\text { I } \\
\text { I } \\
\text { I } \\
\text { I }\end{array}$ & $\begin{array}{rrr}18 & 48 & 59.15 \\
21 & 31 & 52.51 \\
22 & 25 & 34.64 \\
0 & 16 & 22.96 \\
1 & 15 & 18.04\end{array}$ & $\begin{array}{l}+.88 \\
+.92 \\
+.90 \\
+.91 \\
+.96\end{array}$ & $\begin{array}{l}-.02 \\
+.03 \\
+.01 \\
+.03 \\
+.08\end{array}$ & $\begin{array}{r}-.3 \\
.0 \\
-.3 \\
+.6 \\
+.8\end{array}$ & $\begin{array}{l}\mathbf{S} \\
\mathbf{S} \\
\mathbf{S} \\
\mathbf{S}\end{array}$ & $\begin{array}{rrr}-20 & 28 & \\
-\quad 9 & 28 & 14.5 \\
-\quad 4 & 15 & 9.4 \\
+\quad 7 & 1 & 46.7 \\
+12 & 23 & 7.0\end{array}$ & $\begin{array}{l}+4.2 \\
+5.1 \\
+7.0 \\
+5.2\end{array}$ & $\begin{array}{l}-1.2 \\
-1.1 \\
+.8 \\
-.3\end{array}$ & $\begin{array}{l}-1.4 \\
-1.2 \\
+.5 \\
-.8\end{array}$ \\
\hline Dec. & $\begin{array}{l}25.7 \\
26.8 \\
12.3 \\
17.5 \\
19.6\end{array}$ & $\begin{array}{l}\mathrm{Sr} \\
\mathrm{Sr} \\
\mathrm{M} \\
\mathrm{Sr} \\
\mathrm{P}\end{array}$ & W & $\begin{array}{ll}\text { II } \\
\text { II } \\
\text { II }\end{array}$ & $\begin{array}{rrr}10 & 57 & 25.87 \\
11 & 42 & 52.12 \\
0 & 50 & 6.99 \\
6 & 5 & \\
8 & 5 & 11.38\end{array}$ & $\begin{array}{l}+.66 \\
+.68 \\
+.89 \\
+.78\end{array}$ & $\begin{array}{l}+.04 \\
\pm .06 \\
-.03 \\
-.01\end{array}$ & $\begin{array}{r}+.2 \\
+.6 \\
-.4 \\
.0\end{array}$ & 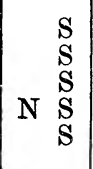 & $\begin{array}{rrr}+ & 057 & 8.5 \\
+3 & 43 & 18.8 \\
+10 & 13 & 28.9 \\
+21 & 48 & 9.4 \\
+16 & 23 & 38.0\end{array}$ & $\begin{array}{r}-2.5 \\
-3.0 \\
+6.2 \\
-2.1\end{array}$ & $\begin{array}{l}+.8 \\
+.7 \\
+.3 \\
\pm .1 \\
-.5\end{array}$ & $\begin{array}{r}+1.0 \\
+1.1 \\
+.4 \\
-.0 \\
-.5\end{array}$ \\
\hline $\begin{array}{c}1919 \\
\text { Jan. }\end{array}$ & \begin{tabular}{r|}
9 \\
6.2 \\
9.2 \\
10.3 \\
13.4
\end{tabular} & $\begin{array}{l}\mathrm{P} \\
\mathrm{Sr} \\
\mathrm{Sr} \\
\mathrm{Sr}\end{array}$ & $\mathbf{E}$ & $\begin{array}{l}\text { I } \\
\text { I } \\
\text { I }\end{array}$ & $\begin{array}{rrr}22 & 45 & 9.80 \\
1 & 28 & 48.93 \\
2 & 27 & 29.08 \\
5 & 36 & \end{array}$ & $\begin{array}{l}+.75 \\
+.81 \\
+.86\end{array}$ & $\begin{array}{l}-.03 \\
=.08 \\
-.07\end{array}$ & $\begin{array}{l}-.4 \\
-1.4 \\
-1.2\end{array}$ & $\mathrm{~N} \stackrel{\stackrel{S}{S}}{\mathrm{~S}}$ & $\begin{array}{rrr}-2 & 18 \\
+13 & 24 & 23.8 \\
+17 & 31 & 32.3 \\
+22 & 16 & 4.9\end{array}$ & $\begin{array}{r}+4.8 \\
+4.0 \\
-\quad .1\end{array}$ & $\begin{array}{r} \\
-.2 \\
-.3 \\
-.8\end{array}$ & $\begin{array}{r}-.1 \\
-.0 \\
-.8\end{array}$ \\
\hline Feb. & $\begin{array}{r}20.6 \\
24.7 \\
6.2 \\
7.2 \\
10.4\end{array}$ & $\begin{array}{l}\mathbf{P} \\
\mathbf{M} \\
\mathbf{M} \\
\mathbf{M} \\
\mathbf{M}\end{array}$ & $\mathbf{E}$ & $\begin{array}{ll} & \text { II } \\
\text { I II } & \end{array}$ & $\begin{array}{rrr}11 & 50 & 54.16 \\
15 & 2 & 40.77 \\
2 & 10 & 6.83 \\
3 & 10 & 12.13 \\
6 & 15 & \end{array}$ & $\begin{array}{l}+.69 \\
+.81 \\
+.83 \\
+.86\end{array}$ & $\begin{array}{l}+.02 \\
+.14 \\
-.02 \\
-.03\end{array}$ & $\begin{array}{l}+.3 \\
+2.2 \\
-.5 \\
-.4\end{array}$ & $\begin{array}{r}\mathbf{S} \\
\mathbf{S} \\
\mathbf{S} \\
\mathrm{S} \\
\mathrm{S}\end{array}$ & $\begin{array}{rrr}-4 & 20 & 33.0 \\
-19 & 19 & 44.0 \\
+16 & 12 & 6.8 \\
+19 & 33 & 34.4 \\
+21 & 25 & 7.4\end{array}$ & $\begin{array}{l}-3.6 \\
-4.0 \\
+4.1 \\
+3.9 \\
-1.1\end{array}$ & $\begin{array}{l}-.1 \\
\bar{t} .9 \\
+.8 \\
\pm .8\end{array}$ & $\begin{array}{l}+.1 \\
\pm .3 \\
+.1 \\
\pm .8 \\
-.8\end{array}$ \\
\hline
\end{tabular}




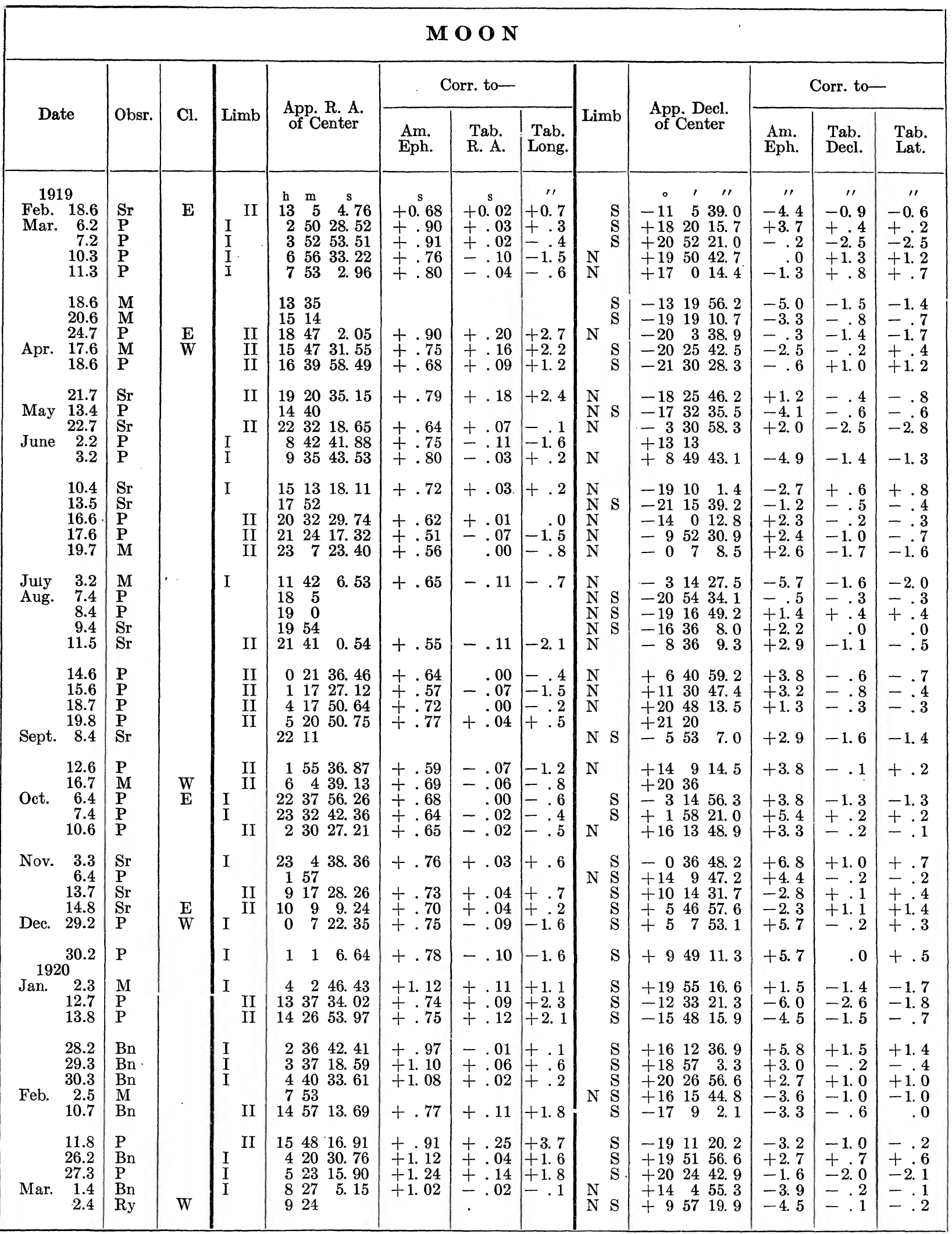


NINE-INCH TRANSIT CIRCLE OBSERVATIONS, 1913-1926

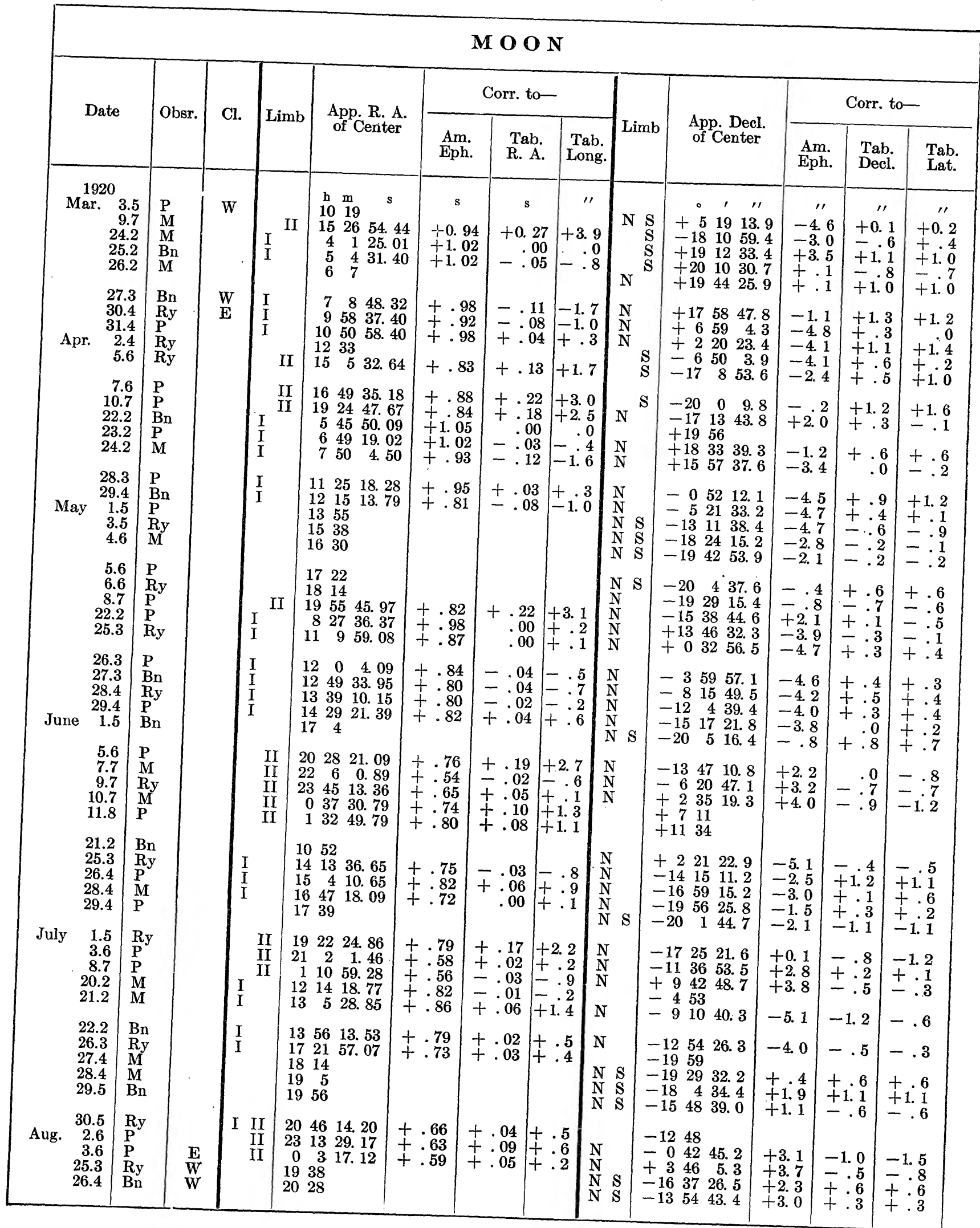


RESULTS OF OBSERVATIONS OF THE SUN, MOON, AND PLANETS

\begin{tabular}{|c|c|c|c|c|c|c|c|c|c|c|c|c|c|}
\hline \multicolumn{14}{|c|}{$\mathbf{M O O N}$} \\
\hline \multirow{2}{*}{\multicolumn{2}{|c|}{ Date }} & \multirow[b]{2}{*}{ Obsr. } & \multirow[b]{2}{*}{ Cl. } & \multirow[b]{2}{*}{ Limb } & \multirow{2}{*}{ App. R. A. } & \multicolumn{3}{|c|}{ Corr. to- } & \multirow[b]{2}{*}{ Limb } & \multirow{2}{*}{$\begin{array}{l}\text { App. Decl. } \\
\text { of Center }\end{array}$} & \multicolumn{3}{|c|}{ Corr. to- } \\
\hline & & & & & & $\begin{array}{l}\text { Am. } \\
\text { Eph. }\end{array}$ & $\begin{array}{l}\text { Tab. } \\
\text { R. A. }\end{array}$ & \begin{tabular}{|c|} 
Tab. \\
Long.
\end{tabular} & & & $\begin{array}{l}\text { Am. } \\
\text { Eph. }\end{array}$ & $\begin{array}{l}\text { Tab. } \\
\text { Decl. }\end{array}$ & $\begin{array}{l}\text { Tab. } \\
\text { Lat. }\end{array}$ \\
\hline $\begin{array}{l}1920 \\
\text { Sept. }\end{array}$ & $\begin{array}{r}0 \\
1.6 \\
2.6 \\
3.7 \\
4.7 \\
16.2\end{array}$ & $\begin{array}{l}\mathrm{Ry} \\
\mathrm{M} \\
\mathrm{M} \\
\mathrm{Bn} \\
\mathrm{Ry}\end{array}$ & W & \begin{tabular}{|l} 
II \\
II \\
II \\
II
\end{tabular} & $\begin{array}{rcc}\mathrm{h} & \mathrm{m} & \mathrm{s} \\
1 & 32 & 5.47 \\
2 & 27 & 42.05 \\
3 & 25 & 52.28 \\
4 & 26 & 25.20 \\
14 & 56 & 39.13\end{array}$ & $\begin{array}{l}\mathrm{s} \\
+0.45 \\
+.45 \\
+.56 \\
+.71 \\
+.95\end{array}$ & $\begin{array}{c}\mathrm{s} \\
-0.17 \\
-.19 \\
-.12 \\
\bar{t} .02 \\
.25\end{array}$ & $\begin{array}{r}\prime \prime \\
-2.7 \\
-2.6 \\
-1.8 \\
-.3 \\
+3.4\end{array}$ & $\begin{array}{l}N \\
N \\
N \\
N\end{array}$ & $\begin{array}{rrr}\circ & \prime & \prime \prime \\
+10 & 50 & 24.8 \\
+14 & 29 & 17.0 \\
+17 & 19 & 54.8 \\
+19 & 7 & 9.1 \\
-15 & 57 & \end{array}$ & $\begin{array}{r}11 \\
+3.6 \\
+4.2 \\
+2.6 \\
+1.5\end{array}$ & $\begin{array}{r}\prime \prime \\
-0.4 \\
+1.0 \\
+.2 \\
+.1\end{array}$ & $\begin{array}{r}1 \prime \\
+0.5 \\
+1.6 \\
+.5 \\
+.1\end{array}$ \\
\hline & $\begin{array}{l}17.2 \\
18.2 \\
20.3 \\
21.3 \\
22.3\end{array}$ & $\begin{array}{l}\mathrm{Ry} \\
\mathrm{P} \\
\mathrm{P} \\
\mathrm{M} \\
\mathrm{M}\end{array}$ & & $\begin{array}{l}\text { I } \\
\text { I } \\
\text { I } \\
\text { I } \\
\text { I }\end{array}$ & $\begin{array}{rrr}15 & 49 & 18.52 \\
16 & 41 & 52.35 \\
18 & 25 & 55.93 \\
19 & 17 & 3.07 \\
20 & 7 & 29.54\end{array}$ & $\begin{array}{l}+.78 \\
+.67 \\
+.99 \\
+.82 \\
+.83\end{array}$ & $\begin{array}{l}+.09 \\
+.01 \\
+.32 \\
+.16 \\
+.18\end{array}$ & $\begin{array}{r}+1.2 \\
.0 \\
+4.5 \\
+2.4 \\
+2.2\end{array}$ & $\begin{array}{cc}\mathbf{N} & \\
\mathbf{S} \\
\mathbf{S} \\
\mathbf{S}\end{array}$ & $\begin{array}{rrr}-18 & 5 \\
-19 & 17 & 4.5 \\
-18 & 52 & 16.0 \\
-17 & 19 & 6.4 \\
-14 & 57 & 16.2\end{array}$ & $\begin{array}{l}-2.2 \\
+2.1 \\
+2.7 \\
+1.8\end{array}$ & $\begin{array}{r}-1.0 \\
+1.6 \\
+1.3 \\
+.7\end{array}$ & $\begin{array}{l}-1.0 \\
+1.4 \\
+1.0 \\
-1.2\end{array}$ \\
\hline Oct. & $\begin{array}{r}25.4 \\
1.6 \\
2.7 \\
4.7 \\
5.7\end{array}$ & $\begin{array}{l}\mathrm{P} \\
\mathrm{M} \\
\mathrm{Ry} \\
\mathrm{P} \\
\mathrm{Bn}\end{array}$ & & $\begin{array}{ll}\text { I } & \\
& \text { II } \\
& \text { II } \\
& \text { II }\end{array}$ & $\begin{array}{rrr}22 & 36 & 48.70 \\
4 & 7 & 55.64 \\
5 & 10 & \\
7 & 14 & 7.07 \\
8 & 14 & 0.65\end{array}$ & $\begin{array}{l}+.69 \\
+.73 \\
+.74 \\
+.61\end{array}$ & $\begin{array}{l}+.07 \\
-.01 \\
-.05 \\
-.18\end{array}$ & $\begin{array}{l}+.6 \\
-.2 \\
-.7 \\
-2.7\end{array}$ & $\underset{N}{N} \begin{array}{l}\text { S } \\
\text { S } \\
\text { S }\end{array}$ & $\begin{array}{rrr} & \\
-4 & 1 & 45.5 \\
+18 & 32 & 36.4 \\
+19 & 27 & 51.1 \\
+17 & 21 & 33.8 \\
+14 & 31 & 36.5\end{array}$ & $\begin{array}{l}+4.0 \\
+2.3 \\
+1.1 \\
-1.8 \\
-2.1\end{array}$ & $\begin{array}{r}-.6 \\
+.4 \\
+.5 \\
+.1 \\
+.9\end{array}$ & $\begin{array}{r}-1.0 \\
+.4 \\
+.5 \\
.0 \\
+.4\end{array}$ \\
\hline & $\begin{array}{r}6.8 \\
16.2 \\
19.2 \\
20.3 \\
21.3\end{array}$ & $\begin{array}{l}\text { Ry } \\
\text { Ry } \\
\text { Ry } \\
\text { Bn } \\
\text { M }\end{array}$ & & $\begin{array}{ll}\text { II } \\
\text { I } \\
\text { I } \\
\text { I } \\
\text { I }\end{array}$ & $\begin{array}{rrr}9 & 11 & 49.68 \\
17 & 12 & 24.03 \\
19 & 46 & 21.41 \\
20 & 35 & 56.60 \\
21 & 25 & 2.34\end{array}$ & $\begin{array}{l}+.70 \\
+.81 \\
+.77 \\
+.76 \\
+.81\end{array}$ & $\begin{array}{l}-.09 \\
+.13 \\
+.11 \\
+.11 \\
+.17\end{array}$ & $\begin{array}{r}-1.7 \\
+1.8 \\
+1.7 \\
+1.2 \\
+2.2\end{array}$ & $\begin{array}{l}\mathbf{S} \\
\mathbf{S} \\
\mathbf{S} \\
\mathbf{S}\end{array}$ & $\begin{array}{rrr}+10 & 48 & 0.6 \\
-19 & 26 & \\
-15 & 55 & 42.7 \\
-13 & 10 & 34.3 \\
-9 & 47 & 25.3\end{array}$ & $\begin{array}{l}-1.5 \\
+3.3 \\
+2.2 \\
+3.8\end{array}$ & $\begin{array}{l}+2.3 \\
+1.3 \\
-.9 \\
-.1\end{array}$ & $\begin{array}{l}+2.0 \\
+1.0 \\
-1.3 \\
-.9\end{array}$ \\
\hline Nov. & $\begin{array}{r}22.3 \\
23.3 \\
25.4 \\
26.4 \\
3.7\end{array}$ & $\begin{array}{l}\text { Ry } \\
\mathrm{Bn} \\
\mathrm{Bn} \\
\mathrm{P} \\
\mathrm{Ry}\end{array}$ & & $\begin{array}{l}\text { I } \\
\text { I } \\
\text { I }\end{array}$ & $\begin{array}{rrr}22 & 14 & 8.04 \\
23 & 3 & 51.37 \\
0 & 48 & 0.46 \\
1 & 44 & \\
9 & 50 & 35.33\end{array}$ & $\begin{array}{l}+.68 \\
+.64 \\
+.67 \\
+.65\end{array}$ & $\begin{array}{l}+.05 \\
+.01 \\
+.03 \\
-.12\end{array}$ & $\begin{array}{l}+.5 \\
+.4 \\
+.3 \\
-2.4\end{array}$ & 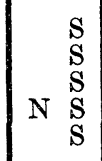 & 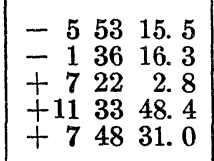 & $\begin{array}{l}+4.6 \\
+6.3 \\
+5.6 \\
+4.3 \\
-1.3\end{array}$ & $\begin{array}{r}.0 \\
+1.2 \\
+\quad .5 \\
+2.7 \\
+2.9\end{array}$ & $\begin{array}{l}-.3 \\
+1.0 \\
+.3 \\
+.6 \\
+2.3\end{array}$ \\
\hline & $\begin{array}{r}4.8 \\
5.7 \\
18.3 \\
19.3 \\
20.3\end{array}$ & $\begin{array}{l}\mathrm{P} \\
\mathrm{Bn} \\
\mathrm{M} \\
\mathrm{Ry} \\
\mathrm{Bn}\end{array}$ & & $\begin{array}{ll} & \text { II } \\
\text { I } & \text { II } \\
\text { I } & \\
\text { I } & \end{array}$ & $\begin{array}{rrr}10 & 44 & 24.49 \\
11 & 36 & 50.67 \\
21 & 52 & 48.84 \\
22 & 41 & 8.49 \\
23 & 30 & 23.52\end{array}$ & $\begin{array}{l}+.73 \\
+.49 \\
+.74 \\
+.68 \\
+.63\end{array}$ & $\begin{array}{r}-.02 \\
-.25 \\
+.07 \\
+.02 \\
-.04\end{array}$ & $\begin{array}{r}-.8 \\
-3.5 \\
+.6 \\
-.2 \\
-.4\end{array}$ & $\begin{array}{l}\mathbf{S} \\
\mathrm{S} \\
\mathrm{S}\end{array}$ & 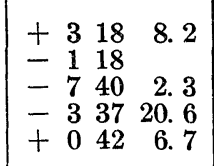 & $\begin{array}{l}-2.4 \\
+3.6 \\
+4.3 \\
+6.3\end{array}$ & $\begin{array}{r}+2.0 \\
-.8 \\
+.7 \\
+.9\end{array}$ & $\begin{array}{l}+1.9 \\
-1.2 \\
-.8 \\
+\quad .9\end{array}$ \\
\hline 192 & 25.5 & $\mathbf{M}$ & W & I II & 41643.45 & +.86 & +.03 & +.4 & & +1844 & & & \\
\hline Jan. & $\begin{array}{r}3.8 \\
17.3 \\
18.3\end{array}$ & $\begin{array}{l}\mathrm{Bn} \\
\mathrm{Ry} \\
\mathrm{P}\end{array}$ & $\mathbf{E}$ & I $^{\text {II }}$ & $\begin{array}{rrr}15 & 23 & 7.19 \\
2 & 15 & 22.08 \\
3 & 12 & 30.58\end{array}$ & $\begin{array}{l}+.63 \\
+.92 \\
+1.04\end{array}$ & $\begin{array}{l}-.02 \\
+.01 \\
+.07\end{array}$ & $\begin{array}{l}-.3 \\
-.2 \\
+.7\end{array}$ & $\begin{array}{l}S \\
S\end{array}$ & $\begin{array}{lll}-16 & 54 & \\
+13 & 13 & 39.7 \\
+16 & 17 & 32.8\end{array}$ & $\begin{array}{l}+4.7 \\
+3.7\end{array}$ & $=.1$ & $\begin{array}{l}-.2 \\
-.4\end{array}$ \\
\hline Feb. & $\begin{array}{l}19.3 \\
23.5 \\
24.6 \\
27.6 \\
14.2\end{array}$ & $\begin{array}{l}\mathrm{P} \\
\mathrm{Ry} \\
\mathrm{Bn} \\
\mathrm{M} \\
\mathrm{M}\end{array}$ & & $\begin{array}{ll}\text { I } & \\
\text { I } & \text { II } \\
& \text { II } \\
& \text { II } \\
\text { I } & \end{array}$ & $\begin{array}{rrr}4 & 13 & 21.96 \\
8 & 34 & 31.24 \\
9 & 36 & 38.41 \\
12 & 27 & 16.11 \\
2 & 52 & 12.61\end{array}$ & $\begin{array}{l}+1.09 \\
+1.02 \\
+.90 \\
+.76 \\
+1.04\end{array}$ & $\begin{array}{r}+.06 \\
+.03 \\
+.03 \\
.00 \\
+.07\end{array}$ & $\begin{array}{r}+.5 \\
+.4 \\
+.5 \\
+.7 \\
+.8\end{array}$ & $\begin{array}{l}\mathbf{S} \\
\mathbf{S} \\
\mathbf{S} \\
\mathbf{S}\end{array}$ & $\begin{array}{rrr}+1826 & 1.2 \\
+13 & 35 & \\
+9 & 20 & 37.5 \\
+5 & 4 & 27.2 \\
+15 & 2 & 15.9\end{array}$ & $\begin{array}{l}+1.4 \\
-3.4 \\
-5.4 \\
+4.5\end{array}$ & $\begin{array}{l}-1.1 \\
+.9 \\
-1.6 \\
+.4\end{array}$ & $\begin{array}{l}-1.2 \\
+.8 \\
-1.3 \\
+.1\end{array}$ \\
\hline & $\begin{array}{l}15.2 \\
16.3 \\
17.3 \\
18.4 \\
25.6\end{array}$ & $\begin{array}{l}\mathrm{P} \\
\mathrm{Bn} \\
\mathrm{Bn} \\
\mathrm{M} \\
\mathrm{Ry}\end{array}$ & & $\begin{array}{l}\text { I } \\
\text { I } \\
\text { I }\end{array}$ & $\begin{array}{rrr}3 & 49 & 42.81 \\
4 & 50 & 9.67 \\
5 & 52 & 56.66 \\
6 & 57 & \\
13 & 50 & \end{array}$ & $\begin{array}{l}+1.14 \\
+1.15 \\
+1.36\end{array}$ & $\begin{array}{r}+.09 \\
+.03 \\
+.21\end{array}$ & $\begin{array}{l}+1.0 \\
+\quad .3 \\
+2.9\end{array}$ & $\begin{array}{r}S \\
S \\
N \underset{S}{S} \\
S\end{array}$ & $\begin{array}{rrr}+17 & 28 & 55.3 \\
+18 & 53 & 52.0 \\
+19 & 4 & \\
+17 & 53 & 4.1 \\
-11 & 12 & 26.1\end{array}$ & $\begin{array}{l}+2.3 \\
+.8 \\
-1.9 \\
-4.7\end{array}$ & $\begin{array}{l}-.6 \\
-.5 \\
+.2 \\
-1.4\end{array}$ & $\begin{array}{l}-.9 \\
-.6 \\
+.2 \\
-1.3\end{array}$ \\
\hline Mar. & $\begin{array}{r}3.7 \\
15.2 \\
16.2 \\
18.3 \\
21.4\end{array}$ & $\begin{array}{l}\mathrm{P} \\
\mathrm{P} \\
\mathrm{Bn} \\
\mathrm{Ry} \\
\mathrm{P}\end{array}$ & & \begin{tabular}{|l} 
II \\
I \\
I \\
I
\end{tabular} & $\begin{array}{rrr}19 & 7 & 2.30 \\
4 & 30 & 54.29 \\
5 & 31 & 33.05 \\
7 & 35 & 0.73 \\
10 & 33 & 51.49\end{array}$ & $\begin{array}{l}+.81 \\
+1.12 \\
+1.22 \\
+1.19 \\
+1.10\end{array}$ & $\begin{array}{l}+.18 \\
+.04 \\
+.08 \\
+.01 \\
+.04\end{array}$ & $\begin{array}{r}+2.5 \\
+.4 \\
+1.0 \\
+.1 \\
+.5\end{array}$ & 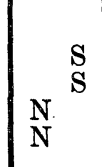 & $\begin{array}{rlr}-17 & 30 & \\
+18 & 22 & 44.6 \\
+18 & 58 & 6.6 \\
+16 & 23 & 13.5 \\
+\quad 4 & 47 & 52.5\end{array}$ & $\begin{array}{l}+1.7 \\
-.7 \\
-2.5 \\
-5.2\end{array}$ & $\begin{array}{l}-.1 \\
\overline{+} .8 \\
+.6\end{array}$ & $\begin{array}{l}-.2 \\
+.9 \\
+1.0 \\
+\quad .9\end{array}$ \\
\hline Apr. & $\begin{array}{l}22.5 \\
25.6 \\
26.6 \\
12.2 \\
15.3\end{array}$ & $\begin{array}{l}\text { M } \\
\text { Ry } \\
. \mathrm{Bn} \\
\mathrm{P} \\
\mathrm{M}\end{array}$ & $\begin{array}{l}\mathrm{E} \\
\mathrm{W} \\
\mathrm{W}\end{array}$ & 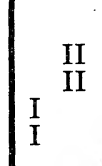 & $\begin{array}{rrr}11 & 31 & \\
14 & 17 & 56.88 \\
15 & 13 & 2.08 \\
5 & 13 & 12.50 \\
8 & 16 & 9.65\end{array}$ & $\begin{array}{l}+.87 \\
+.86 \\
+1.17 \\
+1.15\end{array}$ & $\begin{array}{l}+.03 \\
+.08 \\
+.08 \\
+.01\end{array}$ & $\begin{array}{l}+.8 \\
+1.1 \\
+1.1 \\
-.3\end{array}$ & 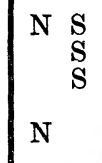 & $\begin{array}{rrr}-0 & 2 & 7.1 \\
-12 & 53 & 47.3 \\
-15 & 48 & 27.5 \\
+18 & 51 & \\
+14 & 22 & 30.0\end{array}$ & $\begin{array}{l}-6.5 \\
-4.3 \\
-2.1 \\
-3.2\end{array}$ & $\begin{array}{l}-.9 \\
\overline{+} .9 \\
+1.2\end{array}$ & $\begin{array}{l}-.8 \\
=.8 \\
+.8 \\
+1.2\end{array}$ \\
\hline
\end{tabular}




\begin{tabular}{|c|c|c|c|c|c|c|c|c|c|c|c|c|c|}
\hline \multicolumn{14}{|c|}{ MOON } \\
\hline \multirow{2}{*}{\multicolumn{2}{|c|}{ Date }} & \multirow[b]{2}{*}{ Obsr. } & \multirow[b]{2}{*}{$\mathrm{Cl}$. } & \multirow[b]{2}{*}{ Limb } & \multirow{2}{*}{$\begin{array}{l}\text { App. R. A. } \\
\text { of Center }\end{array}$} & \multicolumn{3}{|c|}{ Corr. to- } & \multirow[b]{2}{*}{ Limb } & \multirow{2}{*}{$\begin{array}{l}\text { App. Decl. } \\
\text { of Center }\end{array}$} & \multicolumn{3}{|c|}{ Corr. to- } \\
\hline & & & & & & $\begin{array}{l}\text { Am. } \\
\text { Eph. }\end{array}$ & $\begin{array}{l}\text { Tab. } \\
\text { R. A. }\end{array}$ & $\begin{array}{l}\text { Tab. } \\
\text { Long. }\end{array}$ & & & $\begin{array}{l}\text { Am. } \\
\text { Eph. }\end{array}$ & $\begin{array}{l}\text { Tab. } \\
\text { Decl. }\end{array}$ & $\begin{array}{l}\text { Tab. } \\
\text { Lat. }\end{array}$ \\
\hline $\begin{array}{l}192 \\
\text { Apr. }\end{array}$ & $\begin{array}{l}21 \\
18.4 \\
19.4 \\
20.4 \\
21.5 \\
25.6\end{array}$ & $\begin{array}{l}P \\
M \\
\text { Ry } \\
\text { Ry } \\
\text { Ry }\end{array}$ & $\mathrm{W}$ & $\begin{array}{ll}\text { I } & \\
\text { I } & \\
\text { I } & \\
\text { I } & \text { II }\end{array}$ & \begin{tabular}{|ccc}
$\mathrm{h}$ & $\mathrm{m}$ & $\mathrm{s}$ \\
11 & 7 & 34.16 \\
12 & 2 & 37.26 \\
12 & 57 & 24.75 \\
13 & 52 & 18.59 \\
17 & 32 &
\end{tabular} & $\begin{array}{r}\text { s } \\
+1.01 \\
+1.05 \\
+.88 \\
+\quad .92\end{array}$ & $\begin{array}{r}\mathrm{s} \\
-0.06 \\
+.02 \\
-.00 \\
.00\end{array}$ & $\begin{array}{r}11 \\
-0.8 \\
+.5 \\
-1.2 \\
.0\end{array}$ & $\begin{array}{l}\mathrm{N} \\
\mathrm{N} \\
\mathrm{N} \\
\mathrm{N} \mathrm{S}\end{array}$ & 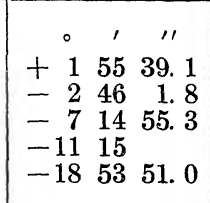 & $\begin{array}{r}11 \\
-5.8 \\
-5.9 \\
-5.2 \\
-.1\end{array}$ & $\begin{array}{r}\quad " \\
+0.3 \\
-.1 \\
-.1 \\
+.4\end{array}$ & 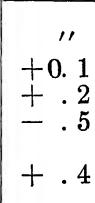 \\
\hline May & $\begin{array}{l}10.2 \\
14.2 \\
16.3 \\
17.3 \\
18.3\end{array}$ & \begin{tabular}{|l}
$\mathrm{P}$ \\
$\mathrm{Ry}$ \\
$\mathrm{P}$ \\
$\mathrm{M}$ \\
$\mathrm{Ry}$
\end{tabular} & & $\begin{array}{l}\text { I } \\
\text { I } \\
\text { I } \\
\text { I } \\
\text { I }\end{array}$ & $\begin{array}{rrr}5 & 53 & 43.55 \\
9 & 54 & 44.05 \\
11 & 44 & 18.02 \\
12 & 37 & 54.74 \\
13 & 31 & 32.60\end{array}$ & $\begin{array}{r}+1.23 \\
+.95 \\
+.91 \\
+.98 \\
+.92\end{array}$ & $\begin{array}{l} \pm .13 \\
\pm .14 \\
=.13 \\
=.02 \\
-.04\end{array}$ & $\begin{array}{l}+1.8 \\
-2.1 \\
-1.8 \\
-.2 \\
-.4\end{array}$ & $\begin{array}{l}\mathrm{N} \\
\mathrm{N} \\
\mathrm{N} \\
\mathrm{N}\end{array}$ & 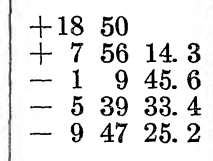 & $\begin{array}{l}-4.9 \\
-5.5 \\
-5.5 \\
-4.9\end{array}$ & $\begin{array}{r}+.8 \\
+.5 \\
+.1 \\
+.1\end{array}$ & $\begin{array}{l}+.1 \\
\pm: 2 \\
+: 2 \\
-.1\end{array}$ \\
\hline & $\begin{array}{l}19.4 \\
20.4 \\
21.5 \\
26.6 \\
31.6\end{array}$ & $\begin{array}{l}\text { Ry } \\
P \\
B n \\
P \\
P\end{array}$ & & II & $\begin{array}{rrr}14 & 25 & 34.20 \\
15 & 20 & 5.06 \\
16 & 15 & \\
20 & 36 & 21.00 \\
0 & 36 & 23.31\end{array}$ & $\begin{array}{l}+.84 \\
+.78 \\
+.51 \\
+.66\end{array}$ & $\begin{array}{l}-.08 \\
-.08 \\
\overline{+} .06 \\
.01\end{array}$ & $\begin{array}{l}-.9 \\
-1.1 \\
-.8 \\
+.1\end{array}$ & $\begin{array}{l}\stackrel{N}{N} \\
\underset{N}{N}\end{array}$ & $\begin{array}{rrr}-13 & 20 & 14.4 \\
-16 & 6 & 55.0 \\
-17 & 59 & 15.7 \\
-13 & 22 & 22.8 \\
+5 & 28 & \end{array}$ & $\begin{array}{l}-4.3 \\
-3.4 \\
-2.7 \\
+2.7\end{array}$ & $\begin{array}{l}-.3 \\
\overline{-} .4 \\
\bar{t} .6 \\
+.4\end{array}$ & $\begin{array}{l}-.5 \\
=.6 \\
+.7 \\
+.5\end{array}$ \\
\hline June & $\begin{array}{l}10.2 \\
14.3 \\
15.3 \\
20.5 \\
21.6\end{array}$ & $\begin{array}{l}\mathrm{M} \\
\mathrm{M} \\
\mathrm{M} \\
\mathrm{Ry} \\
\mathrm{M}\end{array}$ & & $\begin{array}{ll} & \\
\text { I } & \\
\text { I } & \\
\text { I } & \\
& \text { II } \\
& \text { II }\end{array}$ & $\begin{array}{rrr}9 & 36 & 30.30 \\
13 & 15 & 10.95 \\
14 & 8 & 18.82 \\
18 & 36 & 31.95 \\
19 & 28 & 10.57\end{array}$ & $\begin{array}{r}+.88 \\
+.86 \\
+.78 \\
+.80 \\
+.72\end{array}$ & $\begin{array}{l}-.22 \\
=.11 \\
=.15 \\
+.12 \\
+.09\end{array}$ & $\begin{array}{l}-3.1 \\
-1.5 \\
-2.2 \\
+1.7 \\
+1.4\end{array}$ & $\begin{array}{l}\mathrm{N} \\
\mathrm{N} \\
\mathrm{N} \\
\mathrm{N}\end{array}$ & $\begin{array}{l}+928 \\
+8 \\
-82647.9 \\
-121029.7 \\
-181952.0 \\
-1648 \quad 48.7\end{array}$ & $\begin{array}{l}-4.8 \\
-4.1 \\
+1.1 \\
+2.5\end{array}$ & $\begin{array}{r}+.2 \\
+.9 \\
+\quad .9 \\
+1.4\end{array}$ & $\begin{array}{r}+.3 \\
+.3 \\
+.7 \\
+1.2\end{array}$ \\
\hline July & $\begin{array}{l}24.7 \\
26.7 \\
27.6 \\
14.3 \\
16.4\end{array}$ & $\begin{array}{l}\text { Ry } \\
M \\
\text { Bn } \\
\text { Ry } \\
\text { Bn }\end{array}$ & & $\begin{array}{ll} & \text { II } \\
\text { II } \\
\text { II } \\
\text { I }\end{array}$ & $\mid \begin{array}{rrr}21 & 54 & 31.06 \\
23 & 28 & 20.22 \\
0 & 16 & 4.31 \\
15 & 39 & 18.94 \\
17 & 26 & 19.74\end{array}$ & $\begin{array}{l}+.41 \\
+.62 \\
+.67 \\
+.70 \\
+.74\end{array}$ & $\begin{array}{l}-.10 \\
+.11 \\
\pm .13 \\
-.16 \\
-.03\end{array}$ & $\begin{array}{r}-1.6 \\
+1.4 \\
+1.8 \\
-2.1 \\
-.4\end{array}$ & $\begin{array}{l}N \\
N \\
N \\
N \\
N\end{array}$ & 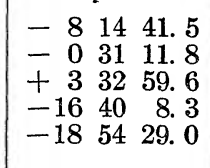 & $\begin{array}{r}+2.9 \\
+4.3 \\
+3.8 \\
-3.0 \\
-1.0\end{array}$ & $\begin{array}{l}-.2 \\
\pm .4 \\
\pm .6 \\
=.6 \\
-.3\end{array}$ & $\begin{array}{l}+.2 \\
\pm .4 \\
=.6 \\
=1.0 \\
-.3\end{array}$ \\
\hline & $\begin{array}{l}17.4 \\
18.4 \\
19.4 \\
20.5 \\
21.6\end{array}$ & $\begin{array}{l}\text { M } \\
\text { M } \\
\text { Ry } \\
\text { Bn } \\
\text { Ry }\end{array}$ & & $\begin{array}{ll}\text { I } & \\
\text { I } & \\
\text { I } & \text { II } \\
& \text { II } \\
& \text { II }\end{array}$ & $\begin{array}{lrr}18 & 19 & 10.38 \\
19 & 11 & 1.53 \\
20 & 1 & 35.85 \\
20 & 50 & 47.87 \\
21 & 38 & 45.78\end{array}$ & $\begin{array}{l}+.84 \\
+.73 \\
+.69 \\
+.58 \\
+.44\end{array}$ & $\begin{array}{l}+.10 \\
+.04 \\
+.03 \\
-.02 \\
-.13\end{array}$ & $\begin{array}{l}+1.4 \\
+.4 \\
+.5 \\
-.4 \\
-1.9\end{array}$ & $\begin{array}{l}\mathrm{N} \\
\mathrm{N} \\
\mathrm{N} \\
\mathrm{N}\end{array}$ & $\begin{array}{rrr}-18 & 36 & 57.3 \\
-17 & 25 & 58.3 \\
-15 & 27 \\
-12 & 47 & 18.9 \\
-9 & 35 & 2.1\end{array}$ & $\begin{array}{r}.0 \\
+.3 \\
+2.3 \\
+3.0\end{array}$ & $\begin{array}{l}-.1 \\
-.6 \\
+.0 \\
+.1\end{array}$ & $\begin{array}{l}-.1 \\
-.7 \\
+.0\end{array}$ \\
\hline Aug. & $\begin{array}{r}23.6 \\
8.2 \\
9.2 \\
10.3 \\
15.4\end{array}$ & $\begin{array}{l}\mathrm{Bn} \\
\mathrm{P} \\
\mathrm{Bn} \\
\mathrm{Bn} \\
\mathrm{P}\end{array}$ & & $\begin{array}{ll} & \text { II } \\
I^{1} & \\
\text { I } & \end{array}$ & $\begin{array}{rrr}23 & 12 & 32.33 \\
13 & 33 & 2.39 \\
14 & 27 & 44.73 \\
15 & 22 & 3.24 \\
19 & 45 & \end{array}$ & $\begin{array}{l}+.52 \\
+.96 \\
+.96 \\
+.76\end{array}$ & $\begin{array}{r}+.01 \\
+.00 \\
+.03 \\
-.13\end{array}$ & $\begin{array}{r}-.2 \\
+.6 \\
+.8 \\
-1.5\end{array}$ & $\begin{array}{l}N \\
N \\
N \\
N \\
N\end{array}$ & $\begin{array}{rrr}-2 & 6 & 16.6 \\
-9 & 16 & 24.5 \\
-12 & 54 & 1.6 \\
-15 & 43 & 13.9 \\
-16 & 6 & 28.9\end{array}$ & $\begin{array}{l}+3.3 \\
-5.4 \\
-4.3 \\
-3.3 \\
+2.6\end{array}$ & $\begin{array}{l}-.4 \\
-1.2 \\
-1.0 \\
\overline{+} .8 \\
.8\end{array}$ & $\begin{array}{l}-.5 \\
-1.0 \\
=.7 \\
-1.2 \\
+.8\end{array}$ \\
\hline & $\begin{array}{l}18.5 \\
19.5 \\
21.6 \\
22.6 \\
23.7\end{array}$ & $\begin{array}{l}\text { Ry } \\
R y \\
R y \\
R y \\
P\end{array}$ & & $\begin{array}{l}\text { II } \\
\text { II } \\
\text { II } \\
\text { II } \\
\text { II }\end{array}$ & $\begin{array}{rrr}22 & 10 & 51.89 \\
22 & 57 & 50.81 \\
0 & 32 & 23.07 \\
1 & 21 & 10.95 \\
2 & 11 & 51.77\end{array}$ & $\begin{array}{l}+.44 \\
+.56 \\
+.48 \\
+.45 \\
+.43\end{array}$ & $\begin{array}{l}=.17 \\
=.03 \\
=.10 \\
=.15 \\
=.20\end{array}$ & $\begin{array}{l}-2.9 \\
-.7 \\
-1.4 \\
-2.1 \\
-3.0\end{array}$ & $\begin{array}{l}\mathrm{N} \\
\mathrm{N} \\
\mathrm{N} \\
\mathrm{N} \\
\mathrm{N}\end{array}$ & 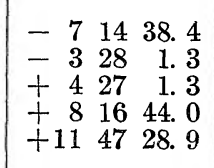 & $\begin{array}{l}+2.5 \\
+3.3 \\
+4.4 \\
+4.4 \\
+3.2\end{array}$ & $\begin{array}{l}-1.1 \\
-.6 \\
+.2 \\
+.4 \\
-.4\end{array}$ & $\begin{array}{l}-.2 \\
\bar{t} .5 \\
+.6 \\
+1.2 \\
+.6\end{array}$ \\
\hline Sept. & $\begin{array}{r}24.7 \\
26.7 \\
7.2 \\
8.2 \\
12.3\end{array}$ & $\begin{array}{l}\mathrm{P} \\
\mathrm{Bn} \\
\mathrm{Bn} \\
\mathrm{Ry} \\
\mathrm{M}\end{array}$ & & $\begin{array}{ll}\underbrace{}_{\text {II }} & \text { II } \\
\text { I } & \\
\text { I }\end{array}$ & $\begin{array}{rrr}3 & 4 & 59.99 \\
4 & 59 & 49.81 \\
15 & 55 & 44.04 \\
16 & 50 & 30.83 \\
20 & 18 & 14.47\end{array}$ & $\begin{array}{l}+.51 \\
+.93 \\
+.81 \\
+.80 \\
+.78\end{array}$ & $\begin{array}{l}-.18 \\
+.12 \\
-.02 \\
.00 \\
+.10\end{array}$ & $\begin{array}{r}-2.7 \\
+1.6 \\
-.2 \\
.0 \\
+1.4\end{array}$ & $\begin{array}{l}\mathrm{N} \\
\mathrm{N} \\
\mathrm{N} \\
\mathrm{N} \\
\quad \mathrm{S}\end{array}$ & 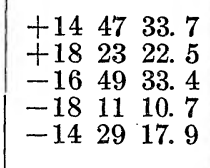 & $\begin{array}{l}+2.9 \\
+1.3 \\
-2.2 \\
-2.9 \\
+2.8\end{array}$ & $\begin{array}{l}\bar{T} .1 \\
\pm: 4 \\
\pm .5 \\
+.1 \\
+.3\end{array}$ & $\begin{array}{l}+.6 \\
\pm .2 \\
\pm .5 \\
-.1 \\
-.1\end{array}$ \\
\hline & $\begin{array}{l}13.4 \\
14.4 \\
15.5 \\
22.7 \\
23.7\end{array}$ & $\begin{array}{l}\mathrm{P} \\
\mathrm{Bn} \\
\mathrm{Bn} \\
\mathrm{M} \\
\mathrm{Ry}\end{array}$ & & II & $\begin{array}{rrr}21 & 6 & 59.86 \\
21 & 55 \\
22 & 42 & \\
4 & 40 & 26.99 \\
5 & 40 & \end{array}$ & $\begin{array}{l}+.69 \\
+.80\end{array}$ & $\begin{array}{l}+.04 \\
-.04\end{array}$ & $\begin{array}{l}+.3 \\
-.7\end{array}$ & $\begin{array}{ll} & S \\
& S \\
N & S \\
N & S \\
N & S\end{array}$ & $\begin{array}{rrr}-11 & 4234.1 \\
-8 & 25 & 13.1 \\
-4 & 45 & 12.5 \\
+17 & 56 & 23.4 \\
+18 & 30 & 27.6\end{array}$ & $\begin{array}{l}+2.9 \\
+2.9 \\
+3.6 \\
+1.4 \\
+.3\end{array}$ & $\begin{array}{r}-.3 \\
-1.0 \\
-.7 \\
-.0 \\
-.2\end{array}$ & $\begin{array}{l}-.5 \\
=.9 \\
=.7 \\
\pm .1 \\
-.2\end{array}$ \\
\hline Oct. & $\begin{array}{r}5.2 \\
6.2 \\
7.2 \\
8.3 \\
14.4\end{array}$ & $\begin{array}{l}\text { Ry } \\
\text { P } \\
\text { Ry } \\
\mathrm{P}\end{array}$ & $\begin{array}{l}\mathrm{W} \\
\mathrm{E}\end{array}$ & $\begin{array}{l}\text { I } \\
\text { I } \\
\text { I } \\
\text { I } \\
\text { I }\end{array}$ & $\begin{array}{lrr}16 & 26 & 46 . \\
17 & 22 & 16.63 \\
18 & 16 & 22.65 \\
19 & 8 & 50.01 \\
23 & 59 & 22.32\end{array}$ & $\begin{array}{l}+.73 \\
+.86 \\
+.84 \\
+.85 \\
+.65\end{array}$ & $\begin{array}{l}\mp .05 \\
+.11 \\
+.11 \\
+.15 \\
+.03\end{array}$ & $\begin{array}{r}+.6 \\
+1.5 \\
+1.6 \\
+2.4 \\
+.6\end{array}$ & $\begin{array}{l}\underset{S}{S} \\
\stackrel{S}{S}\end{array}$ & $\begin{array}{ll}-1738 \\
-18 \quad 26 \\
-181542.3 \\
-17 \quad 1044.0 \\
+\quad 140 & 27.9\end{array}$ & $\begin{array}{l}+.6 \\
+3.1 \\
+5.7\end{array}$ & $\begin{array}{r}.0 \\
+1.7 \\
+\quad .8\end{array}$ & $\begin{array}{r}.0 \\
+1.4 \\
+\quad .5\end{array}$ \\
\hline
\end{tabular}




\begin{tabular}{|c|c|c|c|c|c|c|c|c|c|c|c|c|c|}
\hline \multicolumn{14}{|c|}{$\mathbf{M O O N}$} \\
\hline \multirow{2}{*}{\multicolumn{2}{|c|}{ Date }} & \multirow[b]{2}{*}{ Obsr. } & \multirow[b]{2}{*}{ Cl. } & \multirow[b]{2}{*}{$\operatorname{Limb}$} & \multirow{2}{*}{$\begin{array}{l}\text { App. R. A. } \\
\text { of Center }\end{array}$} & \multicolumn{3}{|c|}{ Corr. to- } & \multirow[b]{2}{*}{ Limb } & \multirow{2}{*}{$\begin{array}{l}\text { App. Decl. } \\
\text { of Center }\end{array}$} & \multicolumn{3}{|c|}{ Corr. to- } \\
\hline & & & & & & Am. & $\begin{array}{l}\text { Tab. } \\
\text { R. A. }\end{array}$ & $\begin{array}{l}\text { Tab. } \\
\text { Long. }\end{array}$ & & & $\begin{array}{l}\text { Am. } \\
\text { Eph. }\end{array}$ & $\begin{array}{l}\text { Tab. } \\
\text { Decl. }\end{array}$ & $\begin{array}{l}\text { Tab. } \\
\text { Lat. }\end{array}$ \\
\hline $\begin{array}{r}1921 \\
\text { Oct. } \\
? \\
? \\
?\end{array}$ & $\begin{array}{l}1 \\
17.5 \\
21.6 \\
22.7 \\
24.7 \\
25.7\end{array}$ & $\begin{array}{l}\text { Bn } \\
R y \\
R y \\
R y \\
M\end{array}$ & $\mathrm{E}$ & $\begin{array}{l}\text { II } \\
\text { II } \\
\text { II } \\
\text { II }\end{array}$ & $\begin{array}{rlc}\mathrm{h} & \mathrm{m} & \mathrm{s} \\
2 & 31 & \\
6 & 21 & 35.04 \\
7 & 21 & 44.04 \\
9 & 20 & 2.57 \\
10 & 17 & 43.22\end{array}$ & $\begin{array}{r}\mathrm{s} \\
+0.98 \\
+.99 \\
+.96 \\
+\quad .83\end{array}$ & $\begin{array}{c}\mathrm{s} \\
+0.02 \\
+.02 \\
+.02 \\
-.08\end{array}$ & $\begin{array}{r}\prime \prime \\
\\
+0.2 \\
+.4 \\
+.1 \\
-1.4\end{array}$ & $\begin{array}{ll}\mathbf{N} & \mathbf{S} \\
\mathbf{S} \\
\mathrm{S} \\
\mathrm{S} \\
\mathrm{S}\end{array}$ & $\begin{array}{ccc}\circ & \prime \prime \\
+12 & 49 & 46.9 \\
+18 & 12 & 15.2 \\
+16 & 47 & 24.1 \\
+10 & 49 & 18.2 \\
+6 & 39 & 36.1\end{array}$ & $\begin{array}{r}\quad l \prime \\
+3.1 \\
+.3 \\
-2.9 \\
-3.6 \\
-4.5\end{array}$ & $\begin{array}{r}11 \\
-1.0 \\
+1.2 \\
+.4 \\
+1.2 \\
+\quad .8\end{array}$ & $\begin{array}{r}1 \prime \\
-0.9 \\
+1.2 \\
+.3 \\
+1.4 \\
+\quad .4\end{array}$ \\
\hline Nov. & $\begin{array}{r}5.2 \\
7.2 \\
10.3 \\
12.4 \\
15.4\end{array}$ & $\begin{array}{l}\mathrm{Bn} \\
\mathrm{M} \\
\mathrm{Bn} \\
\mathrm{Ry} \\
\mathrm{Ry}\end{array}$ & & $\begin{array}{l}\mathrm{I} \\
\mathrm{I} \\
\mathrm{I} \\
\mathrm{I}\end{array}$ & $\begin{array}{rrr}19 & 38 & 41.44 \\
21 & 17 & 37.69 \\
23 & 39 & 20.76 \\
1 & 17 & 8.61 \\
4 & 1 & \end{array}$ & $\begin{array}{l}+.84 \\
+.75 \\
+.65 \\
+.61\end{array}$ & $\begin{array}{l}+.16 \\
+.11 \\
+.05 \\
-.02\end{array}$ & $\begin{array}{l}+2.6 \\
+1.4 \\
+1.0 \\
-\quad .3\end{array}$ & $\begin{array}{r}\mathrm{S} \\
\mathrm{S} \\
\mathrm{S} \\
\mathrm{S} \\
\mathrm{S}\end{array}$ & $\begin{array}{rrr}-16 & 13 & 36.5 \\
-11 & 1 & 44.8 \\
-0 & 2 & 43.8 \\
+7 & 51 & 44.9 \\
+16 & 57 & 41.6\end{array}$ & $\begin{array}{l}+3.9 \\
+2.9 \\
+6.0 \\
+5.2 \\
+2.3\end{array}$ & $\begin{array}{l}+2.2 \\
+.4 \\
+1.2 \\
+.4 \\
-.4\end{array}$ & $\begin{array}{l}+1.8 \\
+.9 \\
+.7 \\
+.4 \\
-.4\end{array}$ \\
\hline Dec. & $\begin{array}{r}3.2 \\
7.2 \\
9.3 \\
15.5 \\
19.7\end{array}$ & $\begin{array}{l}\mathrm{P} \\
\mathrm{P} \\
\mathrm{M} \\
\mathrm{P} \\
\mathrm{P}\end{array}$ & & $\begin{array}{ll}\text { I } & \\
\text { I } & \\
\text { I } & \\
& \text { II } \\
& \text { II }\end{array}$ & $\begin{array}{rrr}20 & 8 & 1.19 \\
23 & 19 & 20.11 \\
0 & 54 & 30.87 \\
6 & 37 & 52.71 \\
10 & 39 & 44.37\end{array}$ & $\begin{array}{l}+.85 \\
+.65 \\
+.65 \\
+.91 \\
+.84\end{array}$ & $\begin{array}{l}+.15 \\
+.02 \\
\pm .01 \\
+.02 \\
-.03\end{array}$ & $\begin{array}{l}+2.1 \\
+.5 \\
+.1 \\
+.4 \\
+.1\end{array}$ & $\begin{array}{l}\mathbf{S} \\
\mathbf{S} \\
\mathbf{S} \\
\mathbf{S}\end{array}$ & 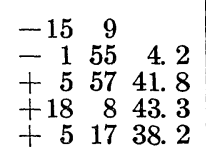 & $\begin{array}{l}+5.8 \\
+5.6 \\
-1.7 \\
-5.8\end{array}$ & $\begin{array}{l}+1.1 \\
+.7 \\
-1.0 \\
-1.1\end{array}$ & $\begin{array}{l}+.8 \\
+.6 \\
-1.0 \\
-1.0\end{array}$ \\
\hline 1922 & $2^{21.7}$ & M & $\mathrm{E}$ & II & $1230 \quad 21.44$ & +.79 & -.06 & -.4 & $\mathrm{~S}$ & -35354.6 & -5.6 & -1.0 & -1.1 \\
\hline Jan. & $\begin{array}{l}6.3 \\
7.3 \\
9.4\end{array}$ & $\begin{array}{l}\mathrm{Bn} \\
\mathrm{Bn} \\
\mathrm{P}\end{array}$ & W & $\begin{array}{l}\text { I } \\
\text { I } \\
\text { I }\end{array}$ & $\begin{array}{rrr}1 & 22 & 30.11 \\
2 & 12 & 50.70 \\
4 & 2 & 38.38\end{array}$ & $\begin{array}{l}+.81 \\
+.80 \\
+\quad .99\end{array}$ & $\begin{array}{l}+.07 \\
+.01 \\
+.08\end{array}$ & $\begin{array}{l}+.9 \\
+.1 \\
+.7\end{array}$ & $\mathbf{S}$ & $\begin{array}{r}+75046.5 \\
+1123 \\
+165015.5\end{array}$ & $\begin{array}{l}+5.2 \\
+1.2\end{array}$ & $\begin{array}{l}+.3 \\
-1.7\end{array}$ & $\begin{array}{l}-.2 \\
-1.9\end{array}$ \\
\hline Feb. & $\begin{array}{r}10.4 \\
12.5 \\
14.6 \\
31.2 \\
2.2\end{array}$ & $\begin{array}{l}\text { Ry } \\
R y \\
M \\
M \\
B n\end{array}$ & & ${ }_{\text {I }}^{\text {I }}$ & $\begin{array}{rrr}5 & 2 & 34.01 \\
7 & 9 & 20.45 \\
9 & 16 & 10.57 \\
23 & 31 & 10.60 \\
1 & 4 & 33.73\end{array}$ & $\begin{array}{l}+1.06 \\
+1.04 \\
+.98 \\
+.70 \\
+.74\end{array}$ & $\begin{array}{l}+.11 \\
+.06 \\
+.08 \\
+.03 \\
+.01\end{array}$ & $\begin{array}{l}+1.4 \\
+\quad .9 \\
+1.4 \\
+.4 \\
-.1\end{array}$ & $\begin{array}{l}S \\
S \\
S\end{array}$ & $\begin{array}{lll}+18 & 16 & 25.2 \\
+17 & 27 & 16.6 \\
+11 & 35 & 36.5 \\
+1 & 27 & \\
+6 & 11 & 53.0\end{array}$ & $\begin{array}{l}+.8 \\
-2.3 \\
-4.6 \\
+4.5\end{array}$ & $\begin{array}{l}-.7 \\
=.6 \\
-.6 \\
-.2\end{array}$ & $\begin{array}{l}-.8 \\
=.4 \\
-.1 \\
-.3\end{array}$ \\
\hline Mar. & $\begin{array}{r}3.2 \\
6.3 \\
8.3 \\
13.6 \\
3.2\end{array}$ & $\begin{array}{l}\text { Ry } \\
\text { P } \\
\text { Ry } \\
\text { Ry } \\
\text { Ry }\end{array}$ & & 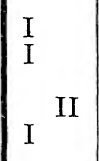 & $\begin{array}{rrr}1 & 53 & 2.00 \\
4 & 33 & 27.36 \\
6 & 35 & \\
11 & 46 & 15.63 \\
2 & 26 & 26.62\end{array}$ & $\begin{array}{l}+.85 \\
+1.10 \\
+.94 \\
+.72\end{array}$ & $\begin{array}{l}+.05 \\
+.05 \\
+.06 \\
-.07\end{array}$ & $\begin{array}{l}+.5 \\
+.5 \\
+1.8 \\
-1.1\end{array}$ & 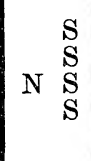 & $\begin{array}{rrr}+9 & 46 & 31.2 \\
+17 & 30 & 14.7 \\
+18 & 4 & 15.1 \\
+0 & 14 & 29.5 \\
+11 & 47 & \end{array}$ & $\begin{array}{l}+4.3 \\
+1.2 \\
-2.4 \\
-6.7\end{array}$ & $\begin{array}{l}-.3 \\
-1.0 \\
-1.3 \\
-2.0\end{array}$ & $\begin{array}{l}-.6 \\
-1.1 \\
-1.3 \\
-1.3\end{array}$ \\
\hline & $\begin{array}{r}5.2 \\
6.2 \\
8.3 \\
12.4 \\
13.4\end{array}$ & $\begin{array}{l}R y \\
P \\
R y \\
R y \\
M\end{array}$ & & $\begin{array}{ll}\text { I } & \\
\text { I } & \\
\text { I } & \\
& \text { II }\end{array}$ & $\begin{array}{rrr}4 & 11 & 46.71 \\
5 & 8 & 14.47 \\
7 & 7 & 43.25 \\
11 & 14 & \\
12 & 13 & 41.01\end{array}$ & $\begin{array}{l}+1.01 \\
+1.09 \\
+1.25 \\
+1.00\end{array}$ & $\begin{array}{r}+.01 \\
+.01 \\
+.03 \\
.00\end{array}$ & $\begin{array}{l}+.2 \\
+.2 \\
+.5 \\
+.4\end{array}$ & $\begin{array}{ll} & S \\
N & S \\
N & S \\
N & S\end{array}$ & $\begin{array}{rrr}+16 & 43 & 33.1 \\
+17 & 59 & 28.0 \\
+17 & 16 & 51.2 \\
+258 & 4.2 \\
+1 & 58 & 5.0\end{array}$ & $\begin{array}{l}+2.8 \\
+1.3 \\
-2.4 \\
-6.9 \\
-6.3\end{array}$ & $\begin{array}{l}+.2 \\
+.2 \\
-1.0 \\
-1.0\end{array}$ & $\begin{array}{l}+.1 \\
+.2 \\
+.1 \\
+.9 \\
-.8\end{array}$ \\
\hline Apr. & $\begin{array}{l}15.6 \\
16.6 \\
17.7 \\
22.7 \\
12.5\end{array}$ & $\begin{array}{l}\mathrm{Ry} \\
\mathrm{P} \\
\mathrm{Bn} \\
\mathrm{Bn} \\
\mathrm{Ry}\end{array}$ & $\underset{\mathrm{W}}{\mathrm{W}}$ & $\begin{array}{l}\text { II } \\
\text { II } \\
\text { II } \\
\text { II }\end{array}$ & $\begin{array}{rrr}14 & 12 & 19.59 \\
15 & 10 & 57.61 \\
16 & 8 & 56.08 \\
20 & 37 & 34.40 \\
14 & 41 & \end{array}$ & $\begin{array}{l}+.91 \\
+.69 \\
+.75 \\
+.61\end{array}$ & $\begin{array}{l}+.06 \\
+.08 \\
+.04 \\
+.06\end{array}$ & $\begin{array}{l}+1.5 \\
+1.0 \\
+\quad .7 \\
+\quad .9\end{array}$ & $\begin{array}{l}\mathbf{S} \\
\mathbf{S} \\
\mathbf{S}\end{array}$ & $\begin{array}{lll}-10 & 52 & 26.1 \\
-14 & 13 & 48.3 \\
-16 & 36 & 13.3 \\
-13 & 46 & \\
-12 & 36 & 44.6\end{array}$ & $\begin{array}{l}-5.5 \\
-2.8 \\
-2.4 \\
-5.1\end{array}$ & $\begin{array}{l}-2.2 \\
-.6 \\
-1.3 \\
-1.6\end{array}$ & $\begin{array}{l}-1.7 \\
-.8 \\
-1.1 \\
-1.6\end{array}$ \\
\hline May & $\begin{array}{r}15.6 \\
1.2 \\
2.2 \\
6.3 \\
8.3\end{array}$ & $\begin{array}{l}\mathrm{P} \\
\mathrm{P} \\
\mathrm{M} \\
\mathrm{Bn} \\
\mathrm{M}\end{array}$ & & $\begin{array}{l}\text { I } \\
\text { I } \\
\text { I } \\
\text { I }\end{array}$ & $\begin{array}{rrrr}17 & 38 & \\
6 & 27 & 41.03 \\
7 & 26 & 23.52 \\
11 & 17 & 44.76 \\
13 & 12 & 45.82\end{array}$ & $\begin{array}{l}+1.24 \\
+1.02 \\
+1.11 \\
+.96\end{array}$ & $\begin{array}{r}+.17 \\
-.09 \\
.00 \\
-.10\end{array}$ & $\begin{array}{l}+2.5 \\
-1.2 \\
-\quad .2 \\
-1.1\end{array}$ & $\begin{array}{ll}\mathrm{N} & \mathrm{S} \\
\mathrm{N} & \\
\mathrm{N} & \\
\mathrm{N} & \end{array}$ & $\begin{array}{r}-181334.6 \\
+185 \\
+164912.8 \\
+24152.6 \\
+63813.7\end{array}$ & $\begin{array}{l}+.3 \\
-3.3 \\
-5.7 \\
-5.9\end{array}$ & $\begin{array}{l}+.2 \\
-.6 \\
+.7 \\
-.4\end{array}$ & $\begin{array}{l}+.2 \\
-.7 \\
+.8 \\
-.8\end{array}$ \\
\hline & $\begin{array}{r}9.4 \\
10.4 \\
15.6 \\
19.7 \\
29.2\end{array}$ & $\begin{array}{l}\mathrm{P} \\
\mathrm{Bn} \\
\mathrm{Bn} \\
\mathrm{Ry} \\
\mathrm{P}\end{array}$ & & $\begin{array}{ll}\text { I } & \\
\text { I } & \text { II } \\
& \text { II } \\
& \text { II } \\
\text { I } & \end{array}$ & $\begin{array}{rrr}14 & 11 & 20.07 \\
15 & 10 & 41.34 \\
19.57 & 45.95 \\
23 & 12 & 28.49 \\
7 & 7 & 58.72\end{array}$ & $\begin{array}{l}+.99 \\
+.97 \\
+.71 \\
+.46 \\
+.98\end{array}$ & $\begin{array}{r}-.03 \\
.00 \\
+.10 \\
-.04 \\
-.13\end{array}$ & $\begin{array}{l}-.6 \\
-.1 \\
+1.6 \\
-.6 \\
-1.8\end{array}$ & $\begin{array}{l}\mathrm{N} \\
\mathrm{N} \\
\mathrm{N}\end{array}$ & $\begin{array}{rrr}-10 & 48 & 50.2 \\
-14 & 15 & \\
-15 & 46 & 35.2 \\
-3 & 12 & 33.1 \\
+17 & 29 & \end{array}$ & $\begin{array}{l}-4.1 \\
+2.9 \\
+3.8\end{array}$ & $\begin{array}{r}+.4 \\
+1.0 \\
.0\end{array}$ & $\begin{array}{l}+.3 \\
+.7 \\
+.1\end{array}$ \\
\hline June & $\begin{array}{r}6.3 \\
7.4 \\
10.5 \\
12.6 \\
15.6\end{array}$ & $\begin{array}{l}\mathrm{P} \\
\mathrm{Ry} \\
\mathrm{P} \\
\mathrm{Bn} \\
\mathrm{M}\end{array}$ & $\mathrm{E}$ & $\begin{array}{ll}\text { I } & \\
\text { I } & \\
& \text { II } \\
& \text { II } \\
& \text { II }\end{array}$ & $\begin{array}{rrr}14 & 45 & 54.62 \\
15 & 44 & 18.80 \\
18 & 39 & 4.67 \\
20 & 27 & 27.18 \\
22 & 53 & 57.02\end{array}$ & $\begin{array}{l}+1.02 \\
+.82 \\
+.83 \\
+.63 \\
+.42\end{array}$ & $\begin{array}{l}+.02 \\
\pm .14 \\
+.06 \\
+.01 \\
-.05\end{array}$ & $\begin{array}{l}+.2 \\
-2.0 \\
+\quad .9 \\
+\quad .3 \\
-\quad .9\end{array}$ & $\begin{array}{l}N \\
N \\
N \\
N\end{array}$ & $\begin{array}{rrr}-12 & 52 & \\
-15 & 45 & 31.0 \\
-18 & 6 & 35.9 \\
-14 & 38 & 54.1 \\
-4 & 56 & 54.9\end{array}$ & $\begin{array}{l}-2.3 \\
+\quad .2 \\
+2.9 \\
+3.0\end{array}$ & $\begin{array}{l}+.4 \\
\pm .4 \\
+1.0 \\
-.3\end{array}$ & $\begin{array}{r}.0 \\
-.5 \\
+\quad .8 \\
.0\end{array}$ \\
\hline
\end{tabular}

$137506^{\circ}-33 \longrightarrow 9$ 


\begin{tabular}{|c|c|c|c|c|c|c|c|c|c|c|c|c|c|}
\hline \multicolumn{14}{|c|}{ MOON } \\
\hline \multirow{2}{*}{\multicolumn{2}{|c|}{ Date }} & \multirow[b]{2}{*}{ Obsr. } & \multirow[b]{2}{*}{$\mathrm{Cl}$. } & \multirow[b]{2}{*}{$\operatorname{Limb}$} & \multirow[b]{2}{*}{$\begin{array}{l}\text { App. R. A. } \\
\text { of Center }\end{array}$} & \multicolumn{3}{|c|}{ Corr. to- } & \multirow[b]{2}{*}{ Limb } & \multirow{2}{*}{$\begin{array}{l}\text { App. Decl. } \\
\text { of Center }\end{array}$} & \multicolumn{3}{|c|}{ Corr. to- } \\
\hline & & & & & & $\begin{array}{l}\text { Am. } \\
\text { Eph. }\end{array}$ & $\begin{array}{l}\text { Tab. } \\
\text { R. A. }\end{array}$ & $\begin{array}{l}\text { Tab. } \\
\text { Long. }\end{array}$ & & & $\begin{array}{l}\text { Am. } \\
\text { Eph. }\end{array}$ & $\begin{array}{l}\text { Tab. } \\
\text { Decl. }\end{array}$ & $\begin{array}{l}\text { Tab. } \\
\text { Lat. }\end{array}$ \\
\hline $\begin{array}{l}192 \\
\text { June } \\
\text { July }\end{array}$ & $\begin{array}{r}22 \\
30.2 \\
5.4 \\
6.4 \\
7.5 \\
12.6\end{array}$ & $\begin{array}{l}\mathrm{Bn} \\
\mathrm{Ry} \\
\mathrm{Ry} \\
\mathrm{Bn} \\
\mathrm{Bn}\end{array}$ & $\mathrm{W}$ & $\begin{array}{ll}\text { I } & \\
\text { I } & \\
\text { I } & \\
& \text { II }\end{array}$ & $\begin{array}{ccc}\mathrm{h} & \mathrm{m} & \mathrm{s} \\
11 & 40 & \\
16 & 21 & 38.52 \\
17 & 19 & 17.45 \\
18 & 16 & 24.16 \\
22 & 35 & 34.06\end{array}$ & $\begin{array}{c}\mathrm{s} \\
+0.87 \\
+.87 \\
+.90 \\
+\quad .54\end{array}$ & $\begin{array}{c}\mathrm{s} \\
-0.07 \\
+.02 \\
+.06 \\
+.04\end{array}$ & $\begin{array}{r}\prime \prime \\
-0.9 \\
-.2 \\
+.9 \\
+.7\end{array}$ & $\begin{array}{l}\mathbf{N} \\
\mathbf{N} \\
\mathbf{N} \\
\mathrm{N} \\
\mathbf{N}\end{array}$ & 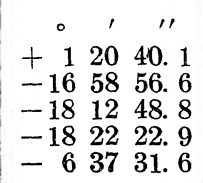 & $\begin{array}{l}11 \\
-7.2 \\
-2.3 \\
-1.2 \\
+.1 \\
+3.3\end{array}$ & $\begin{array}{r}11 \\
-1.3 \\
-.6 \\
-.5 \\
-.2 \\
+.5\end{array}$ & $\begin{aligned} & \prime \prime \\
&-1 . 2 \\
&-\quad .7 \\
&-.5 \\
&-\quad .2 \\
&+.1\end{aligned}$ \\
\hline Aug. & \begin{tabular}{r|}
31.3 \\
2.3 \\
4.4 \\
5.4 \\
9.6
\end{tabular} & $\begin{array}{l}\mathrm{Bn} \\
\mathrm{Ry} \\
\mathrm{Bn} \\
\mathrm{P} \\
\mathrm{M}\end{array}$ & W & $\begin{array}{ll}\text { I } & \\
\text { I } & \\
& \\
& \text { II }\end{array}$ & $\begin{array}{rrr}15 & 7 & 19.77 \\
17 & 1 & 7.51 \\
18 & 53 & \\
19 & 47 & \\
23 & 5 & 20.59\end{array}$ & $\begin{array}{l}+.80 \\
+.78 \\
+.54\end{array}$ & $\begin{array}{l}-.19 \\
-.13 \\
+.03\end{array}$ & $\begin{array}{l}-2.7 \\
-1.8 \\
+.8\end{array}$ & $\begin{array}{ll}\mathrm{N} & \\
\mathrm{N} & \\
\mathrm{N} & \mathrm{S} \\
\mathrm{N} & \mathrm{S} \\
\mathrm{N} & \end{array}$ & $\begin{array}{rrr}-1339 & 18.3 \\
-17 & 47 & 16.7 \\
-17 & 50 & 56.3 \\
-1626 & 20.0 \\
-425 & 43.1\end{array}$ & $\begin{array}{l}-2.9 \\
-1.7 \\
+1.8 \\
+1.5 \\
+4.2\end{array}$ & $\begin{array}{r}.0 \\
-1.0 \\
+\quad .7 \\
+1.3 \\
+1.0\end{array}$ & $\begin{array}{l}-.7 \\
-1.2 \\
+.7 \\
+.2 \\
+.6\end{array}$ \\
\hline Sept. & $\begin{array}{r}28.2 \\
29.3 \\
30.3 \\
5.4 \\
6.5\end{array}$ & $\begin{array}{l}\mathrm{Bn} \\
\mathrm{M} \\
\mathrm{Ry} \\
\mathrm{M} \\
\mathrm{M}\end{array}$ & $\mathbf{E}$ & $\begin{array}{ll}\text { I } & \\
\text { I } & \\
\text { I } & \\
\text { I } & \text { II } \\
& \text { II }\end{array}$ & $\begin{array}{rrr}15 & 46 & 5.70 \\
16 & 43 & 40.87 \\
17 & 40 & 31.21 \\
22 & 49 & 23.92 \\
23 & 36 & 4.13\end{array}$ & $\begin{array}{l}+.84 \\
+.82 \\
+.85 \\
+.57 \\
+.59\end{array}$ & $\begin{array}{l}=.12 \\
=.10 \\
=.02 \\
=.01 \\
+.05\end{array}$ & $\begin{array}{l}-1.5 \\
-1.4 \\
-.3 \\
-.2 \\
+.3\end{array}$ & $\begin{array}{l}\mathrm{N} \\
\mathrm{N} \\
\mathrm{N} \\
\mathrm{N}\end{array}$ & $\begin{array}{rrr}-15 & 19 & 58.1 \\
-17 & 15 & 25.1 \\
-18 & 8 & 0.8 \\
-5 & 43 & \\
-\quad 1 & 59 & 9.7\end{array}$ & $\begin{array}{l}-2.7 \\
-1.8 \\
+.1 \\
+2.9\end{array}$ & $\begin{array}{l}-.8 \\
-1.0 \\
-.2 \\
-.8\end{array}$ & $\begin{array}{r}-1.0 \\
-1.2 \\
-.2 \\
-1.1\end{array}$ \\
\hline & $\begin{array}{l}12.7 \\
13.7 \\
15.7 \\
25.2 \\
26.2\end{array}$ & $\begin{array}{l}\mathrm{P} \\
\mathrm{M} \\
\mathrm{Ry} \\
\mathrm{P} \\
\mathrm{M}\end{array}$ & & $\begin{array}{ll} & \text { II } \\
& \text { II } \\
\text { I I } & \end{array}$ & $\begin{array}{rrr}4 & 28 & 5.59 \\
5 & 23 & 2.12 \\
7 & 19 & 1.87 \\
16 & 21 & 44.19 \\
17 & 20 & 28.93\end{array}$ & $\begin{array}{l}+.61 \\
+.83 \\
+.98 \\
+.77 \\
+.88\end{array}$ & $\begin{array}{l}-.05 \\
+.10 \\
+.12 \\
+.13 \\
+.02\end{array}$ & $\begin{array}{l}-.8 \\
+1.4 \\
+1.7 \\
-1.8 \\
+.3\end{array}$ & $\begin{array}{l}\mathrm{N} \\
\mathrm{N}\end{array}$ & $\begin{array}{l}+164714.0 \\
+175535.5 \\
+179 \\
-1635 \\
-175324.2\end{array}$ & $\begin{array}{l}+1.7 \\
+1.0 \\
-.1\end{array}$ & $\begin{array}{r}.0 \\
+.4 \\
-.1\end{array}$ & $\begin{array}{l}+.1 \\
+.3 \\
-.1\end{array}$ \\
\hline Oct. & $\begin{array}{r}27.2 \\
28.3 \\
29.3 \\
30.3 \\
2.4\end{array}$ & $\begin{array}{l}\text { Ry } \\
R y \\
M \\
P \\
P\end{array}$ & & $\begin{array}{l}\text { I } \\
\text { I } \\
\text { I } \\
\text { I } \\
\text { I }\end{array}$ & $\begin{array}{rrr}18 & 17 & 42.45 \\
19 & 12 & 59.02 \\
20 & 6 & 6.09 \\
20 & 57 & 6.56 \\
22 & 33 & 58.86\end{array}$ & $\begin{array}{l}+.86 \\
+.86 \\
+.75 \\
+.69 \\
+.56\end{array}$ & $\begin{array}{l}+.04 \\
+.10 \\
+.04 \\
+.03 \\
+.03\end{array}$ & $\begin{array}{l}+.7 \\
+1.7 \\
+.5 \\
+.3 \\
-.2\end{array}$ & $\begin{array}{l}S \\
S \\
S \\
S \\
S \\
S\end{array}$ & $\begin{array}{rrr}-18 & 5 & 46.6 \\
-17 & 17 & 42.2 \\
-15 & 37 & 11.5 \\
-13 & 13 & 14.5 \\
-6 & 52 & 11.9\end{array}$ & $\begin{array}{l}+1.9 \\
+4.0 \\
+1.9 \\
+2.6 \\
+4.3\end{array}$ & $\begin{array}{l}+1.0 \\
+2.2 \\
-.6 \\
+.4 \\
+.6\end{array}$ & $\begin{array}{l}+1.0 \\
+2.0 \\
+.8 \\
+.5 \\
+\quad .6\end{array}$ \\
\hline & $\begin{array}{r}5.5 \\
12.7 \\
24.2 \\
26.2 \\
27.2\end{array}$ & $\begin{array}{l}\mathrm{Ry} \\
\mathrm{P} \\
\mathrm{P} \\
\mathrm{Bn} \\
\mathrm{M}\end{array}$ & & $\begin{array}{ll}\text { I } & \text { II } \\
& \text { II } \\
\text { I } & \\
\text { I } & \\
\text { I } & \end{array}$ & $\begin{array}{rrrr}0 & 53 & 39.45 \\
6 & 56 & 34.87 \\
17 & 53 & 39.36 \\
19 & 46 & 43.62 \\
20 & 39 & 20.46\end{array}$ & $\begin{array}{r}+.60 \\
+.81 \\
+.88 \\
+.77 \\
+.76\end{array}$ & $\begin{array}{l}+.06 \\
+.03 \\
+.09 \\
+.08 \\
+.11\end{array}$ & $\begin{array}{r}+.8 \\
+.4 \\
+1.2 \\
+1.5 \\
+1.4\end{array}$ & $\begin{array}{l}S \\
S \\
S\end{array}$ & $\begin{array}{l}+420 \\
+174018.3 \\
+1813 \\
-162516.7 \\
-141350.4\end{array}$ & $\begin{array}{l}-.7 \\
+4.3 \\
+2.2\end{array}$ & $\begin{array}{l}+.8 \\
+2.3 \\
-.5\end{array}$ & $\begin{array}{l}+.8 \\
+2.0 \\
-\quad .9\end{array}$ \\
\hline Nov. & \begin{tabular}{r|}
28.3 \\
30.3 \\
3.4 \\
8.6 \\
9.7
\end{tabular} & $\begin{array}{l}\mathrm{P} \\
\mathrm{P} \\
\mathrm{P} \\
\mathrm{Ry} \\
\mathrm{P}\end{array}$ & & $\begin{array}{ll}\text { I } & \\
\text { I } & \\
\text { I } & \\
& \text { II } \\
& \text { II }\end{array}$ & $\begin{array}{rrr}21 & 29 & 33.80 \\
23 & 4 & 53.55 \\
2 & 12 & 47.68 \\
6 & 39 & 4.70 \\
7 & 35 & 58.85\end{array}$ & $\begin{array}{r}+.64 \\
+.59 \\
+.57 \\
+.87 \\
+.93\end{array}$ & $\begin{array}{r}+.03 \\
+.04 \\
-.02 \\
.00 \\
+.04\end{array}$ & $\begin{array}{l}+.5 \\
+.8 \\
-.4 \\
+.1 \\
+.3\end{array}$ & $\begin{array}{l}\mathbf{S} \\
\mathbf{S} \\
\mathbf{S} \\
\mathbf{S} \\
\mathbf{S}\end{array}$ & $\begin{array}{rrr}-11 & 24 & 50.7 \\
-4 & 32 & 48.8 \\
+10 & 11 & 45.1 \\
+18 & 5 & 7.5 \\
+16 & 52 & 29.7\end{array}$ & $\begin{array}{l}+3.4 \\
+4.4 \\
+3.9 \\
+.2 \\
-.2\end{array}$ & $\begin{array}{l}+.3 \\
+.6 \\
+.1 \\
+1.1 \\
+2.0\end{array}$ & $\begin{array}{r}.0 \\
+\quad .2 \\
+1.1 \\
+2.1\end{array}$ \\
\hline & $\begin{array}{l}11.7 \\
23.2 \\
24.2 \\
27.3 \\
28.3\end{array}$ & $\begin{array}{l}\mathrm{P} \\
\mathrm{Bn} \\
\mathrm{M} \\
\mathrm{P} \\
\mathrm{M}\end{array}$ & & $\begin{array}{ll}\text { II } \\
\text { I } \\
\text { I }\end{array}$ & $\begin{array}{rrr}9 & 29 & 51.72 \\
20 & 17 & 21.70 \\
21 & 10 & \\
23 & 34 & 4.95 \\
0 & 20 & 19.19\end{array}$ & $\begin{array}{l}+.85 \\
+.74 \\
+.54 \\
+.55\end{array}$ & $\begin{array}{l}-.04 \\
+.08 \\
+.02 \\
+.03\end{array}$ & $\begin{array}{l}-.2 \\
+1.0 \\
+\quad .7 \\
+.9\end{array}$ & $\begin{array}{l}\mathbf{S} \\
\mathbf{S} \\
\mathbf{S} \\
\mathrm{S} \\
\mathbf{S}\end{array}$ & $\begin{array}{rrr}+11 & 30 & 31.7 \\
-15 & 27 & 11.2 \\
-12 & 49 & 28.0 \\
-2 & 23 & 10.9 \\
+ & 126 & 32.4\end{array}$ & $\begin{array}{l}-5.3 \\
+1.3 \\
+2.6 \\
+4.9 \\
+5.1\end{array}$ & $\begin{array}{r}-.9 \\
-.8 \\
+.0 \\
+1.2 \\
+1.2\end{array}$ & $\begin{array}{r}-.9 \\
-1.1 \\
.0 \\
+\quad .9 \\
+\quad .8\end{array}$ \\
\hline Dec. & $\begin{array}{r}29.3 \\
30.4 \\
3.4\end{array}$ & $\begin{array}{l}\text { Ry } \\
\text { Ry } \\
\text { Ry }\end{array}$ & $\mathbf{E}$ & $\begin{array}{ll}\text { I } & \\
\text { I } & \\
\text { I } & \text { II }\end{array}$ & $\begin{array}{lrr}1 & 6 & 52.20 \\
1 & 54 & 26.20 \\
4 & 28 & 15.01\end{array}$ & $\begin{array}{l}+.53 \\
+.63 \\
+\quad .76\end{array}$ & $\begin{array}{r}.00 \\
+.08 \\
+.03\end{array}$ & $\begin{array}{l}+.3 \\
+1.2 \\
+.4\end{array}$ & $\stackrel{\mathbf{S}}{\mathrm{S}}$ & $\begin{array}{r}51338.1 \\
+\quad 84957.2 \\
+\quad 857\end{array}$ & $\begin{array}{l}+4.9 \\
+4.3\end{array}$ & $\begin{array}{r}+.9 \\
+\quad .3\end{array}$ & $\begin{array}{l}+.7 \\
-.2\end{array}$ \\
\hline $\begin{array}{l}1923 \\
\text { Jan. }\end{array}$ & 6.6 & $\mathrm{P}$ & W & II & $1048 \quad 43.59$ & +.43 & -.04 & +.2 & $\mathrm{~s}$ & +62642.3 & -4.1 & -1.9 & -2.1 \\
\hline & $\begin{array}{l}10.8 \\
11.8 \\
22.2 \\
25.3 \\
29.3\end{array}$ & $\begin{array}{l}\mathrm{Bn} \\
\mathrm{M} \\
\mathrm{P} \\
\mathrm{Ry} \\
\mathrm{M}\end{array}$ & & $\begin{array}{ll} & \text { II } \\
\text { II } & \\
\text { I } & \end{array}$ & $\begin{array}{rrr}14 & 33 & 16.95 \\
15 & 31 & 8.03 \\
0 & 29 & 14.82 \\
2 & 51 & 10.93 \\
6 & 27 & 23.55\end{array}$ & $\begin{array}{l}+.47 \\
+.46 \\
+.35 \\
+.48 \\
+.55\end{array}$ & $\begin{array}{l}-.01 \\
-.03 \\
=.04 \\
+.06 \\
+.06\end{array}$ & $\begin{array}{r}+1.2 \\
-.4 \\
-.5 \\
+.9 \\
+.9\end{array}$ & $\begin{array}{l}\mathrm{S} \\
\mathrm{S} \\
\mathrm{S}\end{array}$ & $\begin{array}{rrr}-11 & 8 & 59.9 \\
-14 & 28 & \\
+1 & 38 & 12.3 \\
+12 & 8 & 25.5 \\
+18 & 18 & 38.9\end{array}$ & $\begin{array}{l}-6.0 \\
+1.9 \\
+1.5 \\
-2.0\end{array}$ & $\begin{array}{r}-4.2 \\
.0 \\
.0 \\
-1.8\end{array}$ & $\begin{array}{l}-4.1 \\
+.4 \\
-.2 \\
-1.7\end{array}$ \\
\hline Feb. & $\begin{array}{l}30.5 \\
19.2 \\
21.2 \\
22.2 \\
23.3\end{array}$ & $\begin{array}{l}P \\
P \\
R y \\
R y \\
R y\end{array}$ & W & $\begin{array}{l}I \\
I \\
I \\
I\end{array}$ & $\begin{array}{lll}7 & 27 \\
0 & 57 & 35.77 \\
2 & 31 & 47.03 \\
3 & 20 & 40.50 \\
4 & 11 & 26.75\end{array}$ & $\begin{array}{r}+.40 \\
+.35 \\
+.40 \\
+\quad .39\end{array}$ & $\begin{array}{l}+.01 \\
-.05 \\
-.01 \\
-.05\end{array}$ & $\begin{array}{r}+.2 \\
-1.0 \\
-.5 \\
-.8\end{array}$ & $\begin{array}{r}\mathrm{N} \\
\mathbf{S} \\
\mathbf{S} \\
\mathbf{S} \\
\mathrm{S}\end{array}$ & $\begin{array}{rrr}+1724 & 15.1 \\
+349 & \\
+1046 & 6.9 \\
+13 & 39 & 16.0 \\
+15 & 57 & 40.2\end{array}$ & $\begin{array}{r}.0 \\
+\quad .6 \\
-.0 \\
-.2\end{array}$ & $\begin{array}{l}+.8 \\
-1.0 \\
-1.4 \\
-1.1\end{array}$ & $\begin{array}{l}+.8 \\
-.6 \\
-1.2 \\
-.9\end{array}$ \\
\hline
\end{tabular}




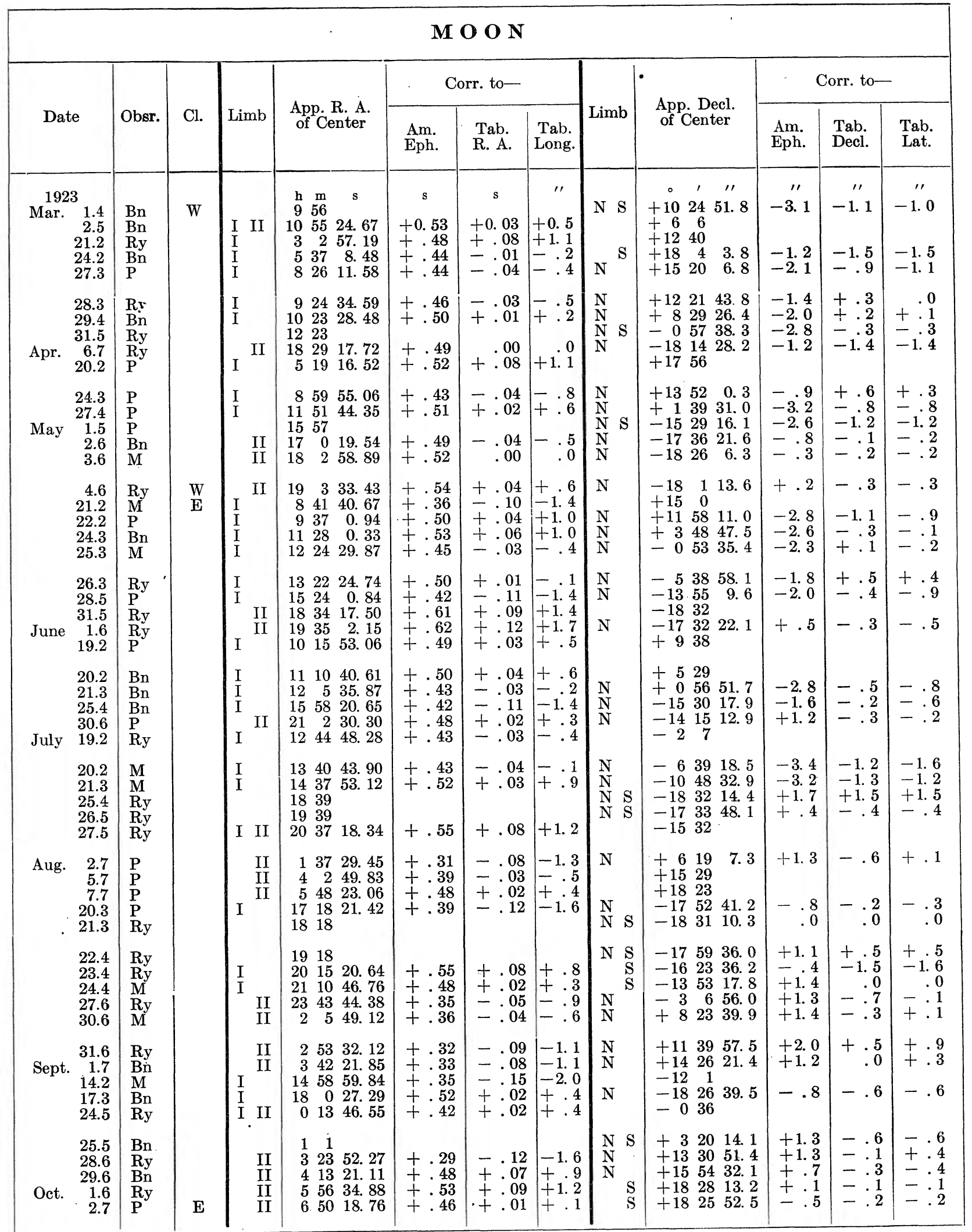




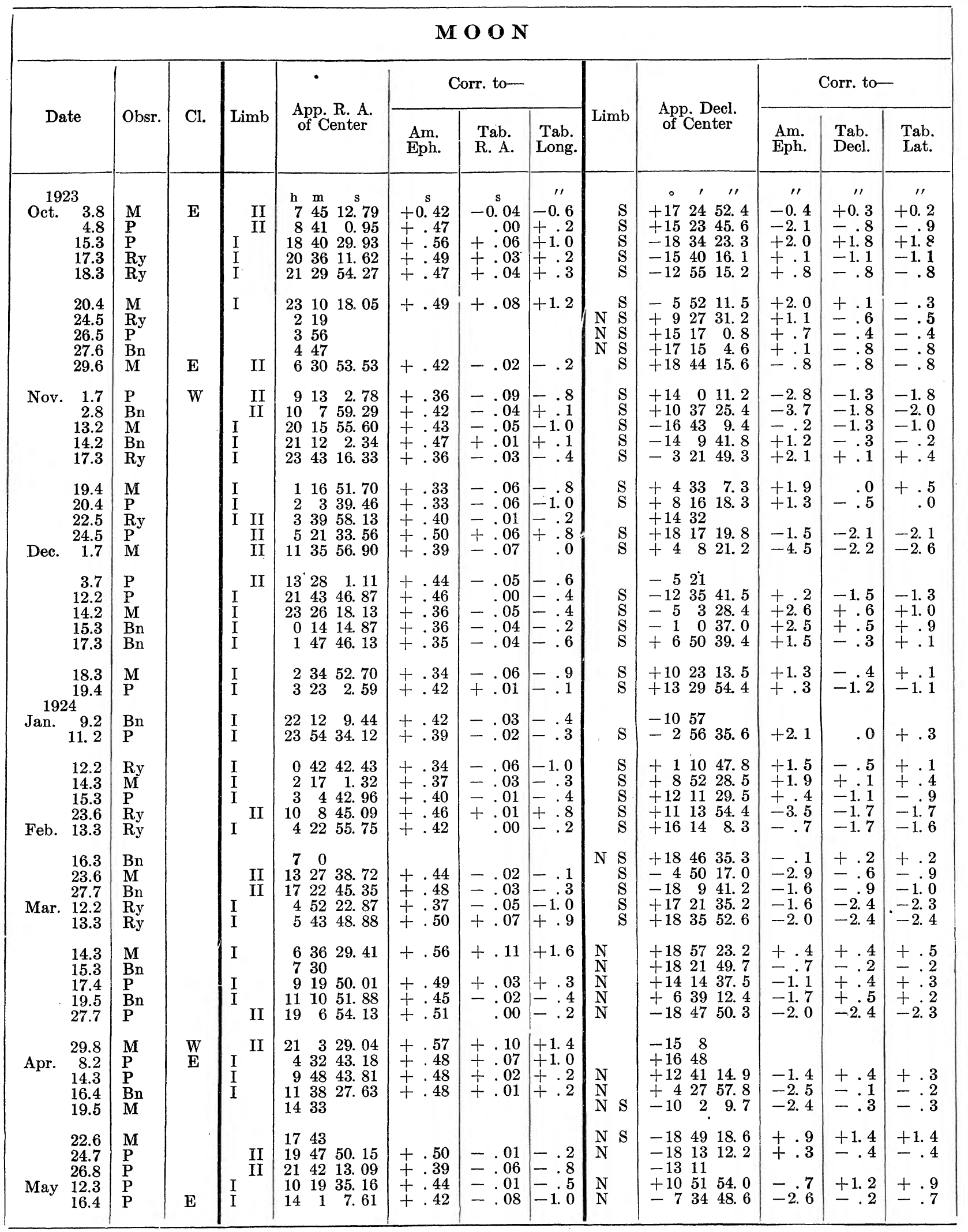




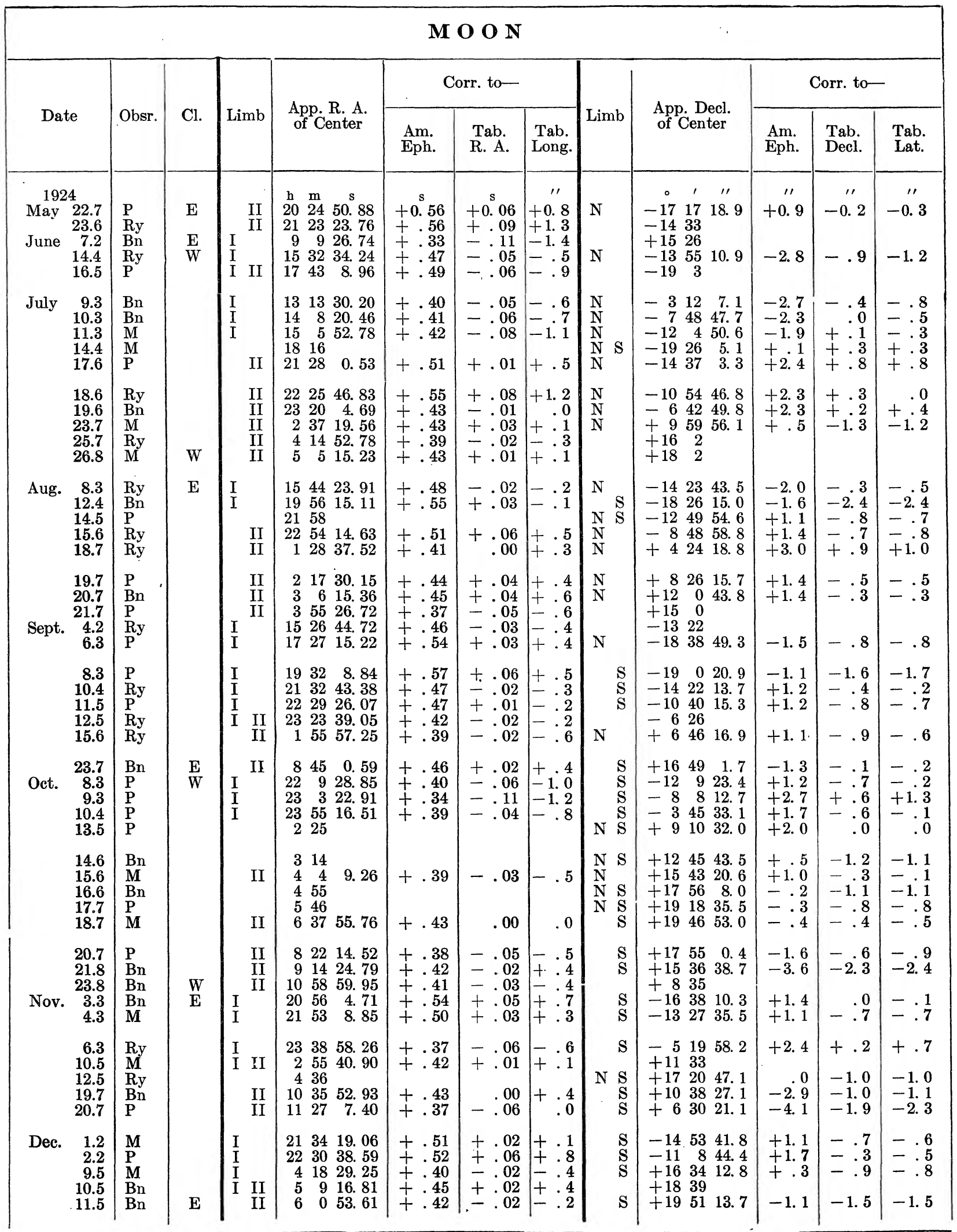




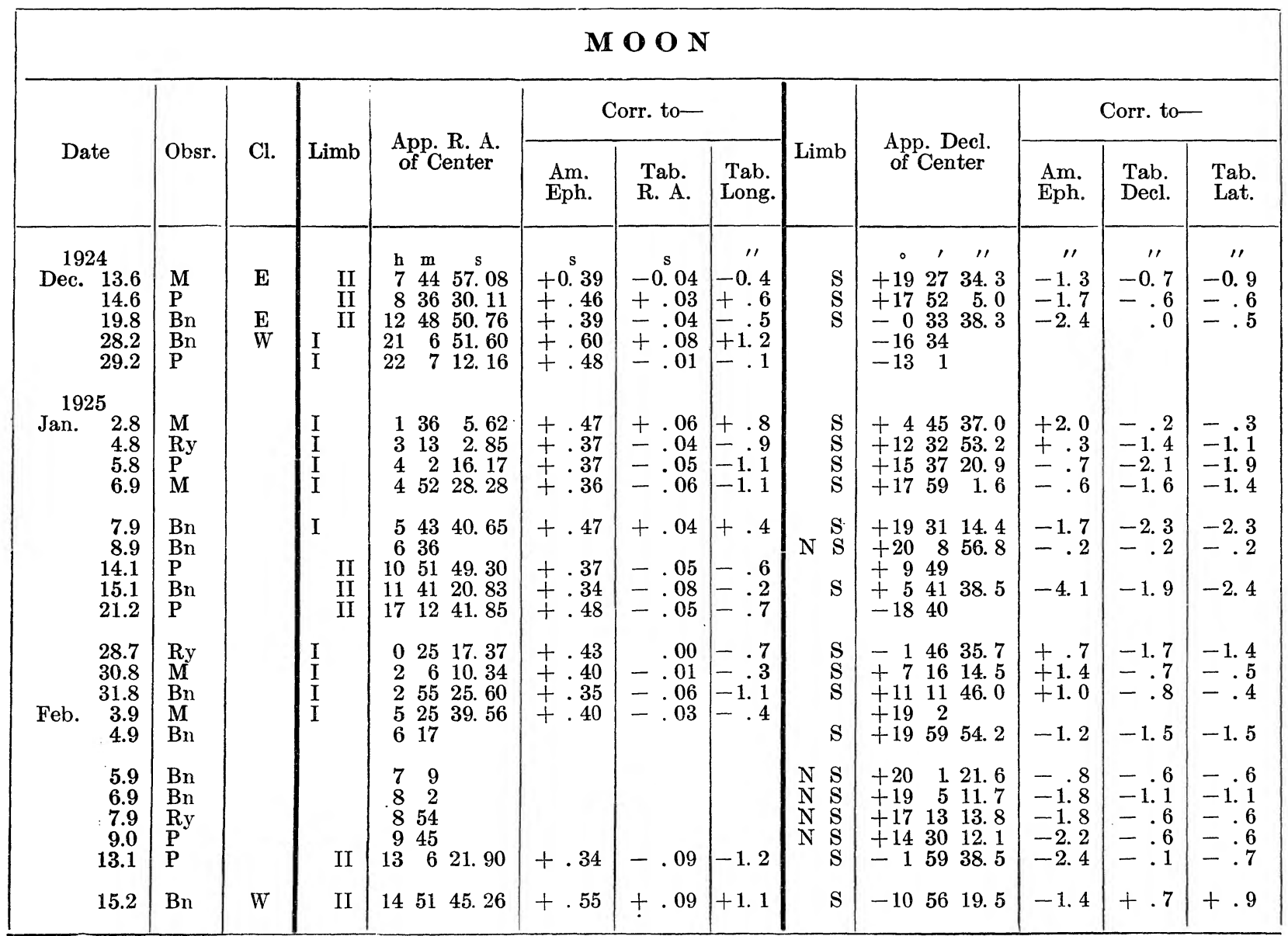


Results of observations of THE sUn, MOON, AND Plianets

\begin{tabular}{|c|c|c|c|c|c|c|c|c|}
\hline \multicolumn{9}{|c|}{$\mathbf{M} \mathbf{E} \mathbb{C} \mathbf{U} \mathbf{Y}$} \\
\hline Date & Obsr. & $\mathrm{Cl}$. & $\operatorname{Limb}$ & $\begin{array}{l}\text { App. R. A. } \\
\text { of Center }\end{array}$ & $\begin{array}{l}\text { Corr. to } \\
\text { Am. Eph. }\end{array}$ & Limb & $\begin{array}{l}\text { App. Decl. } \\
\text { of Center }\end{array}$ & $\begin{array}{l}\text { Corr. to. } \\
\text { Am. Eph. }\end{array}$ \\
\hline \begin{tabular}{lr}
\multicolumn{1}{c}{1918} \\
Sept. 30.0 \\
Oct. $\quad 1.0$ \\
4.0 \\
8.0 \\
10.0
\end{tabular} & $\begin{array}{l}\mathrm{P} \\
\mathrm{P} \\
\mathrm{P} \\
\mathrm{Sr} \\
\mathrm{Sr}\end{array}$ & W & $\begin{array}{l}\text { II } \\
\text { II } \\
\text { II } \\
\text { C } \\
\text { C. }\end{array}$ & $\begin{array}{ccr}\mathrm{h} & \mathrm{m} & \mathrm{s} \\
11 & 45 & 22.958 \\
11 & 51 & 53.883 \\
12 & 11 & 27.697 \\
12 & 37 & 18.250 \\
12 & 50 & 3.168\end{array}$ & $\begin{array}{r}\text { s } \\
+0.118 \\
+\quad .113 \\
+.097 \\
+.240 \\
+.128\end{array}$ & $\begin{array}{l}\mathbf{C} \\
\mathbf{C} \\
\mathbf{C} \\
\mathbf{C} \\
\mathbf{C}\end{array}$ & 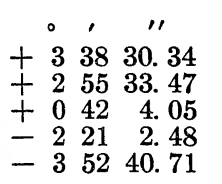 & $\begin{array}{r}\prime \prime \\
-0.56 \\
-.73 \\
-.65 \\
-.58 \\
-1.41\end{array}$ \\
\hline $\begin{array}{r}21.0 \\
\text { Nov. } 7.0 \\
8.0 \\
11.0 \\
14.0\end{array}$ & $\begin{array}{l}\mathbf{P} \\
\mathbf{M} \\
\mathbf{M} \\
\mathbf{P} \\
\mathbf{P}\end{array}$ & & $\begin{array}{l}\mathrm{C} \\
\mathrm{C} \\
\mathbf{C} \\
\mathrm{C} \\
\mathbf{C}\end{array}$ & $\begin{array}{rrr}13 & 58 & 20.923 \\
15 & 42 & 25.847 \\
15 & 48 & 37.124 \\
16 & 7 & 12.778 \\
16 & 25 & 48.053\end{array}$ & $\begin{array}{l}+.173 \\
+.027 \\
+.034 \\
+.088 \\
+.243\end{array}$ & $\begin{array}{l}\mathbf{C} \\
\mathbf{C} \\
\mathbf{C} \\
\mathbf{C} \\
\mathbf{C}\end{array}$ & $\begin{array}{rrr}-11 & 49 & 45.88 \\
-21 & 19 & 2.44 \\
-21 & 44 & 10.03 \\
-22 & 52 & 57.37 \\
-23 & 51 & 27.59\end{array}$ & $\begin{array}{l}+.12 \\
-.54 \\
-1.33 \\
-.27 \\
-1.49\end{array}$ \\
\hline $\begin{array}{c}26.1 \\
29.1 \\
\text { Dec. } 2.1 \\
26.0 \\
1919\end{array}$ & $\begin{array}{l}\mathrm{M} \\
\mathrm{M} \\
\mathrm{P} \\
\mathrm{P}\end{array}$ & W & $\begin{array}{l}\text { C } \\
\text { C } \\
\text { I } \\
\text { II }\end{array}$ & $\begin{array}{rrr}17 & 36 & 38.049 \\
17 & 51 & 39.172 \\
18 & 4 & 12.922 \\
17 & 8 & 44.393\end{array}$ & $\begin{array}{l}+.159 \\
+.042 \\
+.072 \\
+.147\end{array}$ & $\begin{array}{l}\mathrm{C} \\
\mathrm{C} \\
\mathrm{C} \\
\mathrm{C}\end{array}$ & $\begin{array}{lll}-25 & 48 & 57.01 \\
-25 & 46 & 43.06 \\
-25 & 31 & 35.96 \\
-19 & 50 & 45.47\end{array}$ & $\begin{array}{l}+.69 \\
+.56 \\
+.99 \\
+1.13\end{array}$ \\
\hline $\begin{array}{r}\text { Jan. } 5.9 \\
6.9 \\
9.9 \\
15.9 \\
27.0\end{array}$ & $\begin{array}{l}\mathbf{M} \\
\mathbf{M} \\
\mathbf{M} \\
\mathbf{P} \\
\mathbf{M}\end{array}$ & $\mathbf{E}$ & $\begin{array}{l}\text { II } \\
\text { II } \\
\text { II } \\
\text { C } \\
\text { C }\end{array}$ & $\begin{array}{rrr}17 & 26 & 31.108 \\
17 & 30 & 34.370 \\
17 & 44 & 9.558 \\
18 & 15 & 58.585 \\
19 & 23 & 21.681\end{array}$ & $\begin{array}{l}+.088 \\
+.070 \\
+.228 \\
+.185 \\
+.041\end{array}$ & $\begin{array}{l}\mathrm{C} \\
\mathrm{C} \\
\mathrm{C} \\
\mathrm{C} \\
\mathrm{C}\end{array}$ & $\begin{array}{rrr}-21 & 17 & 16.83 \\
-21 & 29 & 20.47 \\
-22 & 4 & 5.00 \\
-22 & 57 & 27.82 \\
-23 & 1 & 25.29\end{array}$ & $\begin{array}{l}-.13 \\
+.33 \\
+.60 \\
+.38 \\
+.11\end{array}$ \\
\hline $\begin{array}{c}30.0 \\
31.0 \\
\text { Feb. } 7.0 \\
18.0 \\
\text { Mar. } 6.0\end{array}$ & $\begin{array}{l}M \\
M \\
P \\
M \\
\text { Sr }\end{array}$ & & $\begin{array}{l}\mathrm{C} \\
\mathrm{C} \\
\mathrm{C} \\
\mathrm{C} \\
\mathrm{C}\end{array}$ & $\begin{array}{lll}19 & 42 & 52.837 \\
19 & 49 & 27.419 \\
20 & 36 & 12.943 \\
21 & 51 & 23.712 \\
23 & 42 & 19.488\end{array}$ & $\begin{array}{l}+.077 \\
+.0109 \\
+.083 \\
+.092 \\
+.258\end{array}$ & $\begin{array}{l}\mathrm{C} \\
\mathrm{C} \\
\mathrm{C} \\
\mathrm{C}\end{array}$ & $\begin{array}{rrr}-22 & 36 & 44.93 \\
-22 & 25 & 54.54 \\
-20 & 31 & 37.56 \\
-15 & 12 & \\
-2 & 38 & 45.29\end{array}$ & $\begin{array}{l}+.47 \\
-1.24 \\
-.36 \\
+1.31\end{array}$ \\
\hline $\begin{array}{r}7.0 \\
10.0 \\
18.0 \\
21.0 \\
25.0\end{array}$ & $\begin{array}{l}\mathrm{Sr} \\
\mathrm{M} \\
\mathrm{P} \\
\mathrm{P} \\
\mathrm{Sr}\end{array}$ & $\mathbf{E}$ & $\begin{array}{l}\text { C } \\
\text { I } \\
\text { I } \\
\text { I } \\
\text { I }\end{array}$ & $\begin{array}{rrr}23 & 49 & 4.463 \\
0 & 8 & 46.093 \\
0 & 53 & 8.447 \\
1 & 4 & 43.734 \\
1 & 14 & 22.940\end{array}$ & $\begin{array}{l}+.193 \\
+.133 \\
-.003 \\
-.016 \\
-.020\end{array}$ & $\begin{array}{l}\mathrm{C} \\
\mathbf{C} \\
\mathbf{C}\end{array}$ & 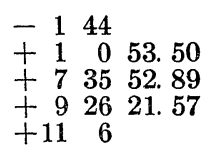 & $\begin{array}{r}-.30 \\
+1.19 \\
+\quad .17\end{array}$ \\
\hline $\begin{array}{rr}\text { Apr. } & 28.9 \\
\text { May } & 1.9 \\
4.9 \\
12.9 \\
\\
14.9\end{array}$ & $\begin{array}{l}\mathbf{P} \\
\mathbf{P} \\
\mathrm{Sr} \\
\mathbf{M} \\
\mathbf{M}\end{array}$ & W & $\begin{array}{l}\text { C } \\
\text { C } \\
\text { II } \\
\text { II } \\
\text { II }\end{array}$ & $\begin{array}{rrr}0 & 51 & 41.393 \\
0 & 59 & 46.707 \\
1 & 9 & 38.830 \\
1 & 43 & 27.668 \\
1 & 53 & 28.664\end{array}$ & $\begin{array}{l}+.113 \\
+.127 \\
+.060 \\
+.088 \\
+.054\end{array}$ & $\begin{array}{l}\text { C } \\
\mathbf{C} \\
\mathbf{C} \\
\mathbf{C} \\
\mathrm{C}\end{array}$ & $\begin{array}{r}+25346.33 \\
+3.2251 .27 \\
+4946.08 \\
+7260.23 \\
+828 \\
+\quad 7.96\end{array}$ & $\begin{array}{l}+.63 \\
-.83 \\
-1.02 \\
-.17 \\
+.46\end{array}$ \\
\hline $\begin{array}{l}18.9 \\
21.9 \\
25.9 \\
27.0 \\
29.0\end{array}$ & $\begin{array}{l}\mathrm{P} \\
\mathrm{P} \\
\mathrm{Sr} \\
\mathrm{Sr} \\
\mathrm{Sr}\end{array}$ & & $\begin{array}{l}\text { II } \\
\text { II } \\
\text { II } \\
\text { II } \\
\text { II }\end{array}$ & $\begin{array}{rrr}2 & 15 & 21.717 \\
2 & 33 & 27.320 \\
2 & 59 & 59.527 \\
3 & 7 & 4.955 \\
3 & 21 & 50.659\end{array}$ & $\begin{array}{l}+.077 \\
+.090 \\
+.087 \\
+.015 \\
+.059\end{array}$ & $\begin{array}{l}\mathrm{C} \\
\mathrm{C} \\
\mathrm{C} \\
\mathrm{C} \\
\mathrm{C}\end{array}$ & $\begin{array}{rrr}+10 & 44 & 14.02 \\
+12 & 34 & 29.02 \\
+15 & 8 & 11.68 \\
+15 & 47 & 9.33 \\
+17 & 4 & 54.69\end{array}$ & $\begin{array}{l}-.48 \\
=.58 \\
=.22 \\
=.67 \\
-.91\end{array}$ \\
\hline 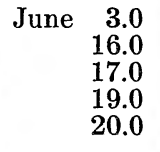 & $\begin{array}{l}\mathrm{M} \\
\mathrm{Sr} \\
\mathrm{Sr} \\
\mathrm{Sr} \\
\mathrm{Sr}\end{array}$ & & $\begin{array}{l}\text { II } \\
\text { I } \\
\text { I } \\
\text { I } \\
\text { I }\end{array}$ & $\begin{array}{rrr}4 & 2 & 11.381 \\
6 & 3 & 29.635 \\
6 & 12 & 55.796 \\
6 & 31 & 27.402 \\
6 & 40 & 31.033\end{array}$ & $\begin{array}{l}+.061 \\
+.155 \\
+.326 \\
+.122 \\
+.083\end{array}$ & $\begin{array}{l}\mathrm{C} \\
\mathrm{C} \\
\mathrm{C} \\
\mathrm{C} \\
\mathrm{C}\end{array}$ & $\begin{array}{rrr}+20 & 11 & 15.29 \\
+24 & 58 & 35.89 \\
+25 & 2 & 28.07 \\
+25 & 2 & 1.02 \\
+24 & 57 & 50.23\end{array}$ & $\begin{array}{l}+.59 \\
+.39 \\
+.53 \\
+.22 \\
+.63\end{array}$ \\
\hline $\begin{array}{cr} & 23.0 \\
& 30.1 \\
\text { July } & 1.1 \\
& 3.1 \\
\text { Aug. } & 26.0\end{array}$ & $\begin{array}{l}\mathrm{Sr} \\
\mathrm{Sr} \\
\mathrm{P} \\
\mathrm{P} \\
\mathrm{P}\end{array}$ & & $\begin{array}{l}\text { I } \\
\text { I } \\
\text { I } \\
\text { I } \\
\text { II }\end{array}$ & $\begin{array}{lrr}7 & 6 & 43.161 \\
8 & 1 & 11.350 \\
8 & 8 & 9.719 \\
8 & 21 & 29.926 \\
9 & 14 & 3.633\end{array}$ & $\begin{array}{l}+.081 \\
+.100 \\
+.079 \\
+.086 \\
+.057\end{array}$ & $\begin{array}{l}\mathrm{C} \\
\mathrm{C} \\
\mathrm{C} \\
\mathrm{C}\end{array}$ & $\begin{array}{rrr}+24 & 30 & 44.83 \\
+22 & 19 & 25.36 \\
+21 & 54 & 44.37 \\
+21 & 2 & 15.66 \\
+14 & 2 & \end{array}$ & $\begin{array}{r}-.27 \\
+1.26 \\
-.93 \\
-.44\end{array}$ \\
\hline $\begin{array}{r}28.0 \\
\text { Sept. } 12.0 \\
15.0 \\
16.0 \\
18.0\end{array}$ & $\begin{array}{l}\mathrm{M} \\
\mathrm{M} \\
\mathrm{P} \\
\mathrm{P} \\
\mathrm{P}\end{array}$ & W & $\begin{array}{l}\text { II } \\
\text { C } \\
\text { II } \\
\text { II } \\
\text { II }\end{array}$ & $\begin{array}{rrr}9 & 17 & 8.798 \\
10 & 33 & 55.511 \\
10 & 54 & 51.396 \\
11 & 1 & 51.719 \cdot \\
11 & 15 & 48.602\end{array}$ & $\begin{array}{l}+.008 \\
+.061 \\
+.086 \\
+.129 \\
+.008\end{array}$ & $\begin{array}{l}\mathrm{C} \\
\mathrm{C} \\
\mathrm{C} \\
\mathrm{C} \\
\mathrm{C}\end{array}$ & 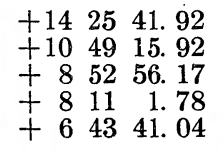 & $\begin{array}{l}+.02 \\
-.78 \\
-1.43 \\
-.32 \\
+.54\end{array}$ \\
\hline 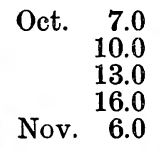 & $\begin{array}{l}\mathbf{M} \\
\mathbf{M} \\
\mathbf{P} \\
\mathbf{P} \\
\mathbf{P}\end{array}$ & $\mathrm{E}$ & $\begin{array}{l}\mathrm{C} \\
\mathrm{C} \\
\mathrm{C} \\
\mathrm{I} \\
\mathrm{C}\end{array}$ & $\begin{array}{rrr}13 & 18 & 7.731 \\
13 & 36 & 0.781 \\
13 & 53 & 42.186 \\
14 & 11 & 15.200 \\
16 & 10 & 42.198\end{array}$ & $\begin{array}{l}+.031 \\
+.001 \\
+.156 \\
+.190 \\
+.078\end{array}$ & $\begin{array}{l}\mathrm{C} \\
\mathrm{C} \\
\mathrm{C} \\
\mathrm{C} \\
\mathrm{C}\end{array}$ & $\begin{array}{rrr}-7 & 52 & 49.02 \\
-10 & 0 & 17.17 \\
-12 & 2 & 5.27 \\
-13 & 57 & 40.38 \\
-23 & 42 & 0.18\end{array}$ & $\begin{array}{r}-.72 \\
-.37 \\
+.03 \\
-.98 \\
-1.38\end{array}$ \\
\hline
\end{tabular}




\begin{tabular}{|c|c|c|c|c|c|c|c|c|}
\hline \multicolumn{9}{|c|}{$M \mathbf{E} C \mathbf{C} \mathbf{Y}$} \\
\hline Date & Obsr. & $\mathrm{Cl}$. & Limb & $\begin{array}{l}\text { App. R. A. } \\
\text { of Center }\end{array}$ & $\begin{array}{l}\text { Corr. to } \\
\text { Am. Eph. }\end{array}$ & Limb & $\begin{array}{l}\text { App. Decl. } \\
\text { of Center }\end{array}$ & $\begin{array}{l}\text { Corr. to. } \\
\text { Am. Eph. }\end{array}$ \\
\hline $\begin{aligned} 1919 \\
\text { Nov. } 10.1 \\
14.1 \\
17.1 \\
18.1 \\
20.0\end{aligned}$ & $\begin{array}{l}\mathrm{P} \\
\mathrm{P} \\
\mathrm{Sr} \\
\mathrm{Sr} \\
\mathrm{P}\end{array}$ & $\mathbf{E}$ & $\begin{array}{l}\text { I } \\
\text { I } \\
\text { I } \\
\text { C } \\
\text { I }\end{array}$ & $\begin{array}{ccc}\mathrm{h} & \mathrm{m} & \mathrm{s} \\
16 & 31 & 2.234 \\
16 & 48 & 41.751 \\
16 & 59 & 2.851 \\
17 & 1 & 43.801 \\
17 & 5 & 38.353\end{array}$ & $\begin{array}{l}\mathrm{s} \\
+0.124 \\
+.021 \\
+.121 \\
+.151 \\
+.057\end{array}$ & $\begin{array}{l}\mathrm{C} \\
\mathrm{C} \\
\mathrm{C} \\
\mathrm{C} \\
\mathrm{C}\end{array}$ & $\begin{array}{rrr}\circ & \prime & \prime \prime \\
-24 & 36 & 3.91 \\
-25 & 6 & 19.59 \\
-25 & 11 & 31.32 \\
-25 & 9 & 38.26 \\
-24 & 59 & 52.56\end{array}$ & $\begin{aligned} & 1 \prime \\
&-1.11 \\
&=.59 \\
&+.18 \\
&-1.86 \\
&-1.76\end{aligned}$ \\
\hline 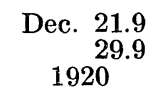 & $\begin{array}{l}\mathrm{Bn} \\
\mathrm{Bn}\end{array}$ & $\mathrm{W}$ & $\begin{array}{l}\text { II } \\
\text { II }\end{array}$ & $\begin{array}{rrr}16 & 26 & 38.561 \\
17 & 7 & 43.547\end{array}$ & $\overline{+} .009$ & $\begin{array}{l}\mathrm{C} \\
\mathrm{C}\end{array}$ & $\begin{array}{lll}-19 & 40 & 17.87 \\
-21 & 57 & 51.89\end{array}$ & $\begin{array}{l}-.97 \\
-1.59\end{array}$ \\
\hline $\begin{array}{ll}\text { Feb. } & 19.0 \\
& 20.0\end{array}$ & $\begin{array}{l}\text { Ry } \\
\text { Ry }\end{array}$ & & $\begin{array}{l}\text { I } \\
\text { I }\end{array}$ & $\begin{array}{lll}22 & 51 & 21.739 \\
22 & 57 & 55.182\end{array}$ & $\begin{array}{l}+.199 \\
+.192\end{array}$ & $\begin{array}{l}\mathrm{C} \\
\mathrm{C}\end{array}$ & $\begin{array}{rrr}-8 & 22 & 8.59 \\
-731 & 50.62\end{array}$ & $\begin{array}{l}+.91 \\
+2.18\end{array}$ \\
\hline $\begin{array}{lr} & 27.1 \\
\text { Mar. } & 1.1 \\
& 2.1 \\
& 8.0 \\
\text { Apr. } & 14.9\end{array}$ & $\begin{array}{l}\mathrm{Ry} \\
\mathrm{M} \\
\mathrm{M} \\
\mathrm{Bn} \\
\mathrm{P}\end{array}$ & $\begin{array}{l}\mathrm{W} \\
\mathbf{E}\end{array}$ & $\begin{array}{l}\text { C } \\
\text { I } \\
\text { I } \\
\text { I } \\
\text { II }\end{array}$ & $\begin{array}{rrr}23 & 39 & 31.151 \\
23 & 53 & 33.411 \\
23 & 57 & 29.985 \\
0 & 11 & 33.713 \\
23 & 56 & 1.436\end{array}$ & $\begin{array}{l}-.009 \\
+.071 \\
+.055 \\
+.003 \\
+.116\end{array}$ & $\begin{array}{l}\text { C } \\
\text { C } \\
\text { C } \\
\text { C. } \\
\text { C }\end{array}$ & 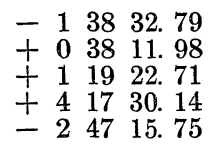 & $\begin{array}{l}+.61 \\
+1.18 \\
+.31 \\
+.64 \\
+.05\end{array}$ \\
\hline $\begin{array}{r}21.9 \\
22.9 \\
28.9 \\
29.9 \\
\text { May } 2.9\end{array}$ & $\begin{array}{l}\text { Ry } \\
\text { Ry } \\
\text { M } \\
\text { M } \\
\text { Bn }\end{array}$ & & $\begin{array}{l}\text { II } \\
\text { II } \\
\text { II } \\
\text { II }\end{array}$ & $\begin{array}{rrr}0 & 24 & 22.714 \\
0 & 28 & 58.896 \\
0 & 59 & 8.222 \\
1 & 4 & 34.720 \\
1 & 21 & 37.079\end{array}$ & $\begin{array}{l}+.084 \\
+.166 \\
+.062 \\
+.130 \\
+.039\end{array}$ & $\begin{array}{l}\mathrm{C} \\
\mathrm{C} \\
\mathrm{C} \\
\mathrm{C} \\
\mathrm{C}\end{array}$ & $\begin{array}{rrrr}- & 0 & 19 & 36.79 \\
+ & 0 & 75.15 \\
+ & 3 & 17 & 37.22 \\
+ & 53 & 20.56 \\
+ & 5 & 46 & 31.88\end{array}$ & $\begin{array}{l}-.79 \\
+.05 \\
+.42 \\
+.46 \\
+.58\end{array}$ \\
\hline $\begin{array}{r}3.9 \\
5.9 \\
10.0 \\
17.0 \\
\text { June } \quad 1.0\end{array}$ & $\begin{array}{l}\mathrm{Bn} \\
\mathrm{Bn} \\
\mathrm{P} \\
\mathrm{Ry} \\
\mathrm{Bn}\end{array}$ & & $\begin{array}{l}\text { II } \\
\text { II } \\
\text { II } \\
\text { II } \\
\text { I }\end{array}$ & $\begin{array}{rrr}1 & 27 & 32.682 \\
1 & 39 & 46.861 \\
2 & 5 & 53.905 \\
2 & 57 & 32.887 \\
5 & 11 & 35.377\end{array}$ & $\begin{array}{l}+.072 \\
+.001 \\
+.165 \\
+.097 \\
+.077\end{array}$ & $\begin{array}{l}\mathrm{C} \\
\mathrm{C} \\
\mathrm{C} \\
\mathrm{C} \\
\mathrm{C}\end{array}$ & $\begin{array}{rrr}+6 & 26 & 6.58 \\
+7 & 47 & 43.25 \\
+10 & 39 & 6.15 \\
+15 & 51 & 49.36 \\
+24 & 27 & 19.79\end{array}$ & $\begin{array}{l}+.78 \\
+.65 \\
+.45 \\
+.96 \\
+.49\end{array}$ \\
\hline $\begin{array}{rr} & 7.0 \\
& 24.1 \\
25.1 \\
29.1 \\
\text { July } \quad 1.1\end{array}$ & $\begin{array}{l}\mathrm{P} \\
\mathrm{M} \\
\mathrm{M} \\
\mathrm{Bn} \\
\mathrm{Bn}\end{array}$ & $\mathbf{E}$ & $\begin{array}{l}\text { I } \\
\text { I } \\
\text { I } \\
\text { I } \\
\text { I }\end{array}$ & $\begin{array}{rrr}6 & 5 & 24.683 \\
8 & 1 & 15.942 \\
8 & 6 & 3.906 \\
8 & 22 & 50.833 \\
8 & 29 & 44.254\end{array}$ & $\begin{array}{l} \pm .053 \\
\pm .008 \\
=.014 \\
=.027 \\
=.076\end{array}$ & $\begin{array}{l}\mathrm{C} \\
\mathrm{C} \\
\mathrm{C} \\
\mathrm{C}\end{array}$ & $\begin{array}{lll}+25 & 24 & 50.62 \\
+21 & 34 & \\
+21 & 10 & 49.16 \\
+19 & 34 & 20.07 \\
+18 & 45 & 50.46\end{array}$ & $\begin{array}{l}+.82 \\
-.04 \\
-2.03 \\
+.66\end{array}$ \\
\hline $\begin{array}{rr}\text { Aug. } & 30.0 \\
\text { Sept. } & 1.0 \\
& 3.0 \\
15.0 \\
17.0\end{array}$ & $\begin{array}{l}\mathrm{M} \\
\mathrm{Bn} \\
\mathrm{Ry} \\
\mathrm{Ry} \\
\mathrm{P}\end{array}$ & $\mathrm{w}$ & $\begin{array}{l}\text { II } \\
\text { II } \\
\text { II } \\
\text { I } \\
\text { I }\end{array}$ & $\begin{array}{rrr}10 & 2 & 41.425 \\
10 & 17 & 49.619 \\
10 & 32 & 46.368 \\
11 & 55 & 27.027 \\
12 & 8 & 3.601\end{array}$ & $\begin{array}{l}+.105 \\
+.029 \\
+.188 \\
+.137 \\
+.141\end{array}$ & $\begin{array}{l}\mathrm{C} \\
\mathrm{C} \\
\mathrm{C} \\
\mathrm{C} \\
\mathrm{C}\end{array}$ & $\begin{array}{rrr}+13 & 46 & 7.61 \\
+12 & 28 & 11.00 \\
+11 & 4 & 23.18 \\
+\quad 1 & 46 & 23 . \\
+ & 03 \\
+ & 11 & 56.60\end{array}$ & $\begin{array}{l}-1.09 \\
+.50 \\
-.02 \\
-1.37 \\
-1.40\end{array}$ \\
\hline $\begin{array}{r}20.0 \\
22.0 \\
24.0 \\
29.0 \\
\text { Oct. } \quad 5.0\end{array}$ & $\begin{array}{l}\text { M } \\
\text { Ry } \\
\text { Ry } \\
\text { My }\end{array}$ & & $\begin{array}{l}\text { I } \\
\text { I } \\
\text { I } \\
\text { I } \\
\text { C }\end{array}$ & $\begin{array}{rrr}12 & 26 & 27.884 \\
12 & 38 & 26.162 \\
12 & 50 & 11.923 \\
13 & 18 & 50.478 \\
13 & 52 & 4.489\end{array}$ & $\begin{array}{l}+.134 \\
+.162 \\
+.183 \\
+.208 \\
+.019\end{array}$ & $\begin{array}{l}\mathbf{C} \\
\mathbf{C} \\
\mathbf{C} \\
\mathbf{C} \\
\mathbf{C}\end{array}$ & 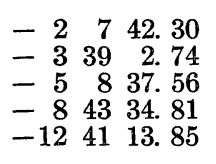 & $\begin{array}{l}-.40 \\
=1.44 \\
=1.36 \\
=1.11 \\
-.15\end{array}$ \\
\hline $\begin{array}{r}7.0 \\
14.0 \\
15.0 \\
22.1 \\
25.1\end{array}$ & $\begin{array}{l}\mathrm{M} \\
\mathrm{Bn} \\
\mathrm{Bn} \\
\mathrm{P} \\
\mathrm{Ry}\end{array}$ & & $\begin{array}{l}\text { C } \\
\text { I } \\
\text { I } \\
\text { I } \\
\text { I }\end{array}$ & $\begin{array}{lrr}14 & 2 & 55.209 \\
14 & 39 & 53.326 \\
14 & 45 & 0.626 \\
15 & 18 & 55.895 \\
15 & 31 & 49.518\end{array}$ & $\begin{array}{l}-.051 \\
+.086 \\
+.106 \\
+.065 \\
+.088\end{array}$ & $\begin{array}{l}\mathbf{C} \\
\mathbf{C} \\
\mathbf{C} \\
\mathbf{C} \\
\mathbf{C}\end{array}$ & $\begin{array}{rrrr}-13 & 54 & 46.80 \\
-17 & 45 & 56.88 \\
-18 & 15 & 15.60 \\
-21 & 9 & 18.75 \\
-22 & 4 & 26.53\end{array}$ & $\begin{array}{l}-.70 \\
=.88 \\
-1.00 \\
-2.35 \\
-.53\end{array}$ \\
\hline 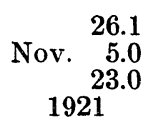 & $\begin{array}{l}\text { Ry } \\
\text { My } \\
\text { Ry }\end{array}$ & W & $\begin{array}{l}\text { I } \\
\text { I }\end{array}$ & $\begin{array}{lll}15 & 35 & 46.952 \\
15 & 58 & 20.212 \\
14 & 58 & 26.027\end{array}$ & $\begin{array}{l}+.062 \\
\pm .138 \\
+.087\end{array}$ & $\begin{array}{l}\mathrm{C} \\
\mathrm{C} \\
\mathbf{C}\end{array}$ & $\begin{array}{rrr}-22 & 19 & 47.84 \\
-23 & 1 & 38.70 \\
-14 & 35 & 25.26\end{array}$ & $\begin{array}{l}-2.14 \\
=.90 \\
-.86\end{array}$ \\
\hline Jan. 25.0 & Ry & $\mathbf{E}$ & I & $2057 \quad 41.052$ & +.152 & C & $-19 \begin{array}{lll}19 & 41.41\end{array}$ & +1.09 \\
\hline $\begin{array}{r}27.0 \\
28.0 \\
\text { Feb. } 4.0 \\
15.1 \\
18.1\end{array}$ & $\begin{array}{l}\mathrm{P} \\
\mathrm{Bn} \\
\mathrm{Ry} \\
\mathrm{Bn} \\
\mathrm{Ry}\end{array}$ & $\mathbf{E}$ & $\begin{array}{l}\text { I } \\
\text { I } \\
\text { I } \\
\text { I } \\
\text { I }\end{array}$ & $\begin{array}{rrr}21 & 11 & 43.573 \\
21 & 18 & 41.724 \\
22 & 5 & 42.293 \\
23 & 1 & 47.200 \\
23 & 8 & 53.025\end{array}$ & $\begin{array}{l}+.113 \\
+.104 \\
+.143 \\
+.000 \\
+.015\end{array}$ & $\begin{array}{l}\mathrm{C} \\
\mathbf{C} \\
\mathbf{C} \\
\mathbf{C} \\
\mathbf{C}\end{array}$ & $\begin{array}{rrr}-18 & 13 & 25.94 \\
-17 & 38 & 34.63 \\
-12 & 57 & 56.79 \\
-5 & 1 & 41.34 \\
-3 & 26 & 30.05\end{array}$ & $\begin{array}{l}+.86 \\
+.67 \\
+1.81 \\
+.66 \\
+.25\end{array}$ \\
\hline
\end{tabular}




\begin{tabular}{|c|c|c|c|c|c|c|c|c|}
\hline \multicolumn{9}{|c|}{$M E R C U Y$} \\
\hline Date & Obsr. & Cl. & Limb & $\begin{array}{l}\text { App. R. A. } \\
\text { of Center }\end{array}$ & $\begin{array}{l}\text { Corr. to } \\
\text { Am. Eph. }\end{array}$ & $\operatorname{Limb}$ & $\begin{array}{l}\text { App. Decl. } \\
\text { of Center }\end{array}$ & $\begin{array}{l}\text { Corr. to. } \\
\text { Am. Eph. }\end{array}$ \\
\hline \begin{tabular}{cc}
\multicolumn{1}{c}{1921} \\
Mar. & 17.9 \\
Apr. & 10.9 \\
& 18.9 \\
May & 19.0 \\
& 20.0
\end{tabular} & $\begin{array}{l}\mathrm{Ry} \\
\mathrm{Bn} \\
\mathrm{Ry} \\
\mathrm{P} \\
\mathrm{Bn}\end{array}$ & $\stackrel{\mathrm{E}}{\mathrm{W}}$ & $\begin{array}{l}\text { II } \\
\text { II } \\
\text { II } \\
\text { I } \\
\text { I }\end{array}$ & \begin{tabular}{rrc}
\multicolumn{1}{c}{} & $\mathrm{m}$ & $\mathrm{s}$ \\
22 & 21 & 35.435 \\
23 & 50 & 54.958 \\
0 & 36 & 17.149 \\
4 & 28 & 43.346 \\
4 & 37 & 28.311
\end{tabular} & $\begin{array}{r}\mathrm{s} \\
-0.015 \\
+.148 \\
+.099 \\
+.034 \\
+.111\end{array}$ & $\begin{array}{l}\mathrm{C} \\
\mathrm{C} \\
\mathrm{C} \\
\mathrm{C}\end{array}$ & 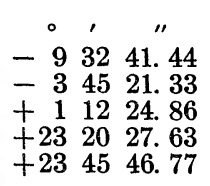 & $\begin{array}{r}\quad " \\
+0.16 \\
+.27 \\
.64 \\
+.13 \\
+.03\end{array}$ \\
\hline $\begin{array}{r}23.0 \\
31.1 \\
\text { June } \\
2.1 \\
3.1 \\
13.1\end{array}$ & $\begin{array}{l}\mathrm{Bn} \\
\mathrm{Ry} \\
\mathrm{P} \\
\mathrm{Bn} \\
\mathrm{Ry}\end{array}$ & & $\begin{array}{l}\text { I } \\
\text { C } \\
\text { I } \\
\text { I } \\
\text { I }\end{array}$ & $\begin{array}{rrr}5 & 3 & 2.995 \\
6 & 3 & 48.330 \\
6 & 16 & 52.287 \\
6 & 23 & 2.930 \\
7 & 10 & 36.494\end{array}$ & $\begin{array}{l}+.135 \\
\pm .150 \\
+.013 \\
+.060 \\
+.014\end{array}$ & $\begin{array}{l}\mathrm{C} \\
\mathrm{C} \\
\mathrm{C} \\
\mathrm{C} \\
\mathrm{C}\end{array}$ & $\begin{array}{r}+244546.12 \\
+253726.50 \\
+252939.45 \\
+2523 \quad 17.17 \\
+231356.02\end{array}$ & $\begin{array}{l}-.18 \\
+.50 \\
+.45 \\
+.27 \\
-.68\end{array}$ \\
\hline $\begin{array}{rr}\text { July } & 26.9 \\
& 31.9 \\
\text { Aug. } & 5.0 \\
10.0 \\
19.0\end{array}$ & $\begin{array}{l}R y \\
R y \\
P \\
\text { Ry } \\
P\end{array}$ & & $\begin{array}{l}\text { II } \\
\text { II } \\
\text { II } \\
\text { II } \\
\text { C }\end{array}$ & $\begin{array}{rrr}7 & 2 & 9.113 \\
7 & 25 & 2.025 \\
7 & 50 & 0.379 \\
8 & 27 & 25.058 \\
9 & 40 & 46.334\end{array}$ & $\begin{array}{l}+.033 \\
+.005 \\
+.159 \\
+.038 \\
+.114\end{array}$ & $\begin{array}{l}\mathrm{C} \\
\mathrm{C} \\
\mathrm{C} \\
\mathrm{C} \\
\mathrm{C}\end{array}$ & 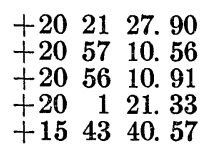 & $\begin{array}{l}+.50 \\
\pm .14 \\
-1.29 \\
+.13 \\
-.83\end{array}$ \\
\hline $\begin{array}{r}31.0 \\
\text { Sept. } \\
2.0 \\
7.0 \\
9.0 \\
13.0\end{array}$ & $\begin{array}{l}\mathrm{Ry} \\
\mathrm{Bn} \\
\mathrm{Ry} \\
\mathrm{Bn} \\
\mathrm{Bn}\end{array}$ & & $\begin{array}{l}\text { I } \\
\text { I } \\
\text { I } \\
\text { I } \\
\text { I }\end{array}$ & $\begin{array}{rrr}11 & 8 & 42.689 \\
11 & 21 & 46 . \\
11 & 52 & 46.194 \\
12 & 4 & 35.083 \\
12 & 27 & 21.554\end{array}$ & $\begin{array}{l}+.129 \\
+.134 \\
+: 104 \\
+.193 \\
+.204\end{array}$ & $\begin{array}{l}\mathrm{C} \\
\mathrm{C} \\
\mathrm{C} \\
\mathrm{C} \\
\mathrm{C}\end{array}$ & 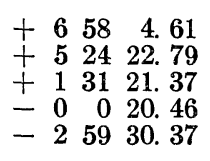 & $\begin{array}{l}-.69 \\
-1.21 \\
-2.13 \\
-1.86 \\
-1.77\end{array}$ \\
\hline $\begin{array}{rr} & 19.0 \\
22.0 \\
23.0 \\
\text { Oct. } \begin{array}{r}4.1 \\
6.1\end{array}\end{array}$ & $\begin{array}{l}\mathrm{Ry} \\
\mathrm{P} \\
\mathrm{Bn} \\
\mathrm{Ry} \\
\mathrm{P}\end{array}$ & $\mathrm{W}$ & $\begin{array}{l}\text { I } \\
\text { I } \\
\text { I } \\
\text { I } \\
\text { I }\end{array}$ & $\begin{array}{rrr}12 & 59 & 41.236 \\
13 & 15 & 7.332 \\
13 & 20 & 9.576 \\
14 & 11 & 18.881 \\
14 & 19 & 27: 010\end{array}$ & $\begin{array}{l}-.094 \\
+.132 \\
+.146 \\
+.041 \\
-.010\end{array}$ & $\begin{array}{l}\mathrm{C} \\
\mathrm{C} \\
\mathrm{C} \\
\mathrm{C} \\
\mathrm{C}\end{array}$ & $\begin{array}{rrr}-7 & 14 & 4.84 \\
-9 & 13 & 27.12 \\
-9 & 51 & 52.68 \\
-15 & 58 & 45.31 \\
-16 & 51 & 49.84\end{array}$ & $\begin{array}{l}-.64 \\
-1.92 \\
-2.08 \\
=.41 \\
-1.54\end{array}$ \\
\hline $\begin{array}{r}17.0 \\
21.0 \\
\text { Nov. } 14.9 \\
21.9 \\
24.9\end{array}$ & $\begin{array}{l}\mathrm{Ry} \\
\mathrm{Bn} \\
\mathrm{Ry} \\
\mathrm{Bn} \\
\mathrm{Ry}\end{array}$ & $\mathbf{E}$ & $\begin{array}{l}\text { I } \\
\text { C } \\
\text { II } \\
\text { II }\end{array}$ & $\begin{array}{rrr}14 & 50 & 7.137 \\
14 & 51 & \\
14 & 8 & 18.786 \\
14 & 39 & 39.111 \\
14 & 55 & 55.576\end{array}$ & $\begin{array}{l}+.057 \\
+.156 \\
+.051 \\
+.046\end{array}$ & $\begin{array}{l}\mathrm{C} \\
\mathbf{C} \\
\mathrm{C} \\
\mathrm{C} \\
\mathrm{C}\end{array}$ & 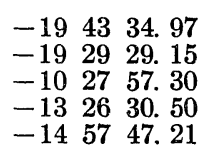 & $\begin{array}{l}-.17 \\
+.35 \\
\pm .60 \\
+.10 \\
+1.59\end{array}$ \\
\hline 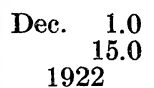 & $\stackrel{\mathrm{P}}{\mathrm{P}}$ & E & $\begin{array}{l}\text { II } \\
\text { C }\end{array}$ & $\begin{array}{rrr}15 & 31 & 8.972 \\
17 & 1 & 10.428\end{array}$ & $\begin{array}{l}+.222 \\
+.188\end{array}$ & $\stackrel{\mathrm{C}}{\mathrm{C}}$ & $\begin{array}{lll}-17 & 58 & 34.34 \\
-23 & 20 & 44.58\end{array}$ & $\begin{array}{l}-.84 \\
-.38\end{array}$ \\
\hline $\begin{array}{rr}\text { Jan. } & 9.0 \\
13.0\end{array}$ & $\begin{array}{l}\mathrm{Ry} \\
\mathrm{Bn}\end{array}$ & W & $\begin{array}{l}\text { I } \\
\text { I }\end{array}$ & $\begin{array}{lll}19 & 56 & 47.806 \\
20 & 24 & 55.821\end{array}$ & $\begin{array}{l}+.196 \\
+.161\end{array}$ & $\begin{array}{l}\mathrm{C} \\
\mathrm{C}\end{array}$ & $\begin{array}{rrr}-22 & 53 & 54.38 \\
-21 & 22 & 6.45\end{array}$ & $\begin{array}{l}-.28 \\
-.45\end{array}$ \\
\hline $\begin{array}{lr} & 26.1 \\
& 30.1 \\
& 31.1 \\
\text { Feb. } & 3.1 \\
\text { Mar. } & 5.9\end{array}$ & $\begin{array}{l}\mathrm{P} \\
\mathrm{Bn} \\
\mathrm{Bn} \\
\mathrm{Ry} \\
\mathrm{Ry}\end{array}$ & & $\begin{array}{l}\text { I } \\
\text { I } \\
\text { I } \\
\text { I } \\
\text { II }\end{array}$ & $\begin{array}{rrr}21 & 45 & 38.048 \\
22 & 1 & 52.763 \\
22 & 4 & 45.726 \\
22 & 9 & 53.497 \\
21 & 24 & 12.199\end{array}$ & $\begin{array}{l}+.128 \\
+.053 \\
+.076 \\
+.007 \\
+.019\end{array}$ & $\begin{array}{l}\mathrm{C} \\
\mathrm{C} \\
\mathrm{C} \\
\mathrm{C} \\
\mathrm{C}\end{array}$ & $\begin{array}{rrr}-14 & 2 & 0.19 \\
-11 & 37 & 17.53 \\
-11 & 4 & 50.06 \\
-9 & 43 & 10.54 \\
-14 & 53 & 22.66\end{array}$ & $\begin{array}{l}+.21 \\
+1.17 \\
+.04 \\
+.94 \\
-.36\end{array}$ \\
\hline $\begin{array}{r}8.9 \\
12.9 \\
15.9 \\
16.9 \\
22.9\end{array}$ & $\begin{array}{l}\mathrm{P} \\
\mathrm{Bn} \\
\mathrm{P} \\
\mathrm{Ry} \\
\mathrm{P}\end{array}$ & W & $\begin{array}{l}\text { II } \\
\text { II } \\
\text { II } \\
\text { II } \\
\text { II }\end{array}$ & $\begin{array}{rrr}21 & 33 & 51.900 \\
21 & 49 & 31.208 \\
22 & 2 & 51.953 \\
22 & 7 & 33.809 \\
22 & 37 & 50.445\end{array}$ & $\begin{array}{l}+.180 \\
+.068 \\
+.053 \\
+.069 \\
+.205\end{array}$ & $\begin{array}{l}\mathrm{C} \\
\mathrm{C} \\
\mathrm{C} \\
\mathrm{C} \\
\mathrm{C}\end{array}$ & $\begin{array}{rrr}-14 & 42 & 46.99 \\
-14 & 5 & 52.17 \\
-13 & 22 & 5.20 \\
-13 & 4 & 33.74 \\
-10 & 50 & 10.85\end{array}$ & $\begin{array}{l} \pm .01 \\
\pm .17 \\
=.40 \\
-.54 \\
-.55\end{array}$ \\
\hline $\begin{array}{r}\text { Apr. } \\
\text { May } \\
1.0 \\
2.0 \\
8.0 \\
9.0\end{array}$ & $\begin{array}{l}\mathrm{P} \\
\mathrm{Ry} \\
\mathrm{Ry} \\
\mathrm{Bn} \\
\mathrm{Bn}\end{array}$ & $\mathrm{E}$ & $\begin{array}{l}\text { C } \\
\text { I } \\
\text { I } \\
\text { I } \\
\text { I }\end{array}$ & $\begin{array}{rrr}1 & 35 & 12.222 \\
3 & 4 & 3.074 \\
3 & 12 & 24.376 \\
4 & 1 & 15.680 \\
4 & 9 & 0.203\end{array}$ & $\begin{array}{l}+.252 \\
+.144 \\
+.066 \\
+.110 \\
+.073\end{array}$ & $\begin{array}{l}\mathrm{C} \\
\mathrm{C} \\
\mathrm{C} \\
\mathrm{C} \\
\mathrm{C}\end{array}$ & $\begin{array}{r}+84810.63 \\
+181130.64 \\
+185536.54 \\
+22331423 \\
+23 \quad 0 \quad 51.88\end{array}$ & $\begin{array}{l}+.73 \\
+1.24 \\
+1.04 \\
+1.13 \\
+1.48\end{array}$ \\
\hline $\begin{array}{rr} & 11.0 \\
& 15.1 \\
& 16.1 \\
& 29.1 \\
\text { July } & 2.9\end{array}$ & $\begin{array}{l}\text { P } \\
\text { Ry } \\
\text { Ry } \\
\text { Ry } \\
\text { Bn }\end{array}$ & $\underset{\mathrm{W}}{\mathrm{E}}$ & $\begin{array}{l}\text { I } \\
\text { I } \\
\text { I } \\
\text { I } \\
\text { II }\end{array}$ & $\begin{array}{rrr}4 & 23 & 59.364 \\
4 & 51 & 34.749 \\
4 & 57 & 54.696 \\
5 & 55 & 14.472 \\
5 & 29 & 4.885\end{array}$ & $\begin{array}{l}+.124 \\
+.149 \\
\pm .004 \\
=.058 \\
-.025\end{array}$ & $\begin{array}{l}\mathrm{C} \\
\mathrm{C} \\
\mathrm{C} \\
\mathrm{C} \\
\mathrm{C}\end{array}$ & $\begin{array}{rrr}+23 & 48 & 27.38 \\
+24 & 54 & 11.32 \\
+25 & 451.88 \\
+24 & 31 \\
+19 & 58.78 \\
+19 & 28.70\end{array}$ & $\begin{array}{l}+.78 \\
\pm .18 \\
=.02 \\
=.52 \\
-.80\end{array}$ \\
\hline
\end{tabular}


NINE-INCH TRANSIT CIRCLE OBSERVATIONS, 1913-1926

\begin{tabular}{|c|c|c|c|c|c|c|c|c|}
\hline \multicolumn{9}{|c|}{$\mathbf{M} \mathbf{E} \mathbf{C} \mathbf{U} \mathbf{Y}$} \\
\hline Date & Obsr. & Cl. & Limb & $\begin{array}{l}\text { App. R. A. } \\
\text { of Center }\end{array}$ & $\begin{array}{c}\text { Corr. to Am. } \\
\text { Eph. }\end{array}$ & $\operatorname{Limb}$ & $\begin{array}{l}\text { App. Decl. } \\
\text { of Center }\end{array}$ & $\begin{array}{l}\text { Corr. to. } \\
\text { Am. Eph. }\end{array}$ \\
\hline \begin{tabular}{rr}
\multicolumn{2}{c}{1922} \\
July $\quad 5.9$ \\
6.9 \\
16.9 \\
28.0 \\
31.0
\end{tabular} & $\begin{array}{l}\mathrm{P} \\
\mathrm{Ry} \\
\mathbf{P} \\
\mathrm{Ry} \\
\mathbf{P}\end{array}$ & W & $\begin{array}{l}\text { II } \\
\text { II } \\
\text { II } \\
\text { II } \\
\text { II }\end{array}$ & $\begin{array}{rrr}\mathrm{h} & \mathrm{m} & \mathrm{s} \\
5 & 34 & 23.055 \\
5 & 36 & 51.557 \\
6 & 20 & 55.408 \\
7 & 44 & 43.761 \\
8 & 11 & 4.835\end{array}$ & $\begin{array}{r}\mathrm{s} \\
+0.005 \\
+.013 \\
+.068 \\
+.081 \\
+.125\end{array}$ & $\begin{array}{l}\mathrm{C} \\
\mathrm{C} \\
\mathrm{C} \\
\mathrm{C} \\
\mathrm{C}\end{array}$ & \begin{tabular}{rrr}
\multicolumn{1}{r}{} & \multicolumn{1}{c}{} & \multicolumn{1}{c}{} \\
+19 & 36 & 5.78 \\
+19 & 48 & 4.56 \\
+21 & 57 & 34.84 \\
+22 & 7 & 25.70 \\
+21 & 19 & 17.99
\end{tabular} & $\begin{array}{l} \\
-1.42 \\
-. .24 \\
=.06 \\
-.50 \\
-.51\end{array}$ \\
\hline $\begin{array}{rr}\text { Aug. } & 18.0 \\
21.0 \\
22.0 \\
28.0 \\
\text { Sept. } 5.1\end{array}$ & $\begin{array}{l}\mathrm{Ry} \\
\mathrm{P} \\
\mathrm{P} \\
\mathrm{P} \\
\mathrm{Bn}\end{array}$ & $\mathbf{E}$ & $\begin{array}{l}\text { I } \\
\text { I } \\
\text { I } \\
\text { I } \\
\text { I }\end{array}$ & $\begin{array}{rrr}10 & 34 & 6.523 \\
10 & 53 & 44.452 \\
11 & 0 & 2.643 \\
11 & 35 & 37.390 \\
12 & 17 & 55.458\end{array}$ & $\begin{array}{l}+.123 \\
+.092 \\
+.043 \\
+.090 \\
+.118\end{array}$ & $\begin{array}{l}\mathrm{C} \\
\mathrm{C} \\
\mathrm{C} \\
\mathrm{C} \\
\mathrm{C}\end{array}$ & $\begin{array}{r}+1030 \\
+81.56 \\
+81518.77 \\
+7303.39 \\
+25955.97 \\
+24412.68\end{array}$ & $\begin{array}{l}=.04 \\
=.53 \\
=.41 \\
=.53 \\
-.78\end{array}$ \\
\hline $\begin{array}{l}7.1 \\
14.1 \\
15.1 \\
18.1 \\
26.1\end{array}$ & $\begin{array}{l}\mathrm{Bn} \\
\mathrm{P} \\
\mathrm{Ry} \\
\mathrm{Ry} \\
\mathrm{P}\end{array}$ & & $\begin{array}{l}\text { I } \\
\text { I } \\
\text { I } \\
\text { I } \\
\text { I }\end{array}$ & $\begin{array}{rrr}12 & 27 & 40.844 \\
12 & 59 & 9.210 \\
13 & 3 & 16.440 \\
13 & 14 & 57.399 \\
13 & 39 & 18.785\end{array}$ & $\begin{array}{r}+.254 \\
.000 \\
+.010 \\
+.049 \\
+.115\end{array}$ & $\begin{array}{l}\mathrm{C} \\
\mathrm{C} \\
\mathrm{C} \\
\mathrm{C} \\
\mathrm{C}\end{array}$ & $\begin{array}{rrr}-4 & 5 & 24.52 \\
- & 27 & 50.88 \\
- & 9 & 0.07 \\
-10 & 38 & 14.63 \\
-13 & 55 & 12.52\end{array}$ & $\begin{array}{r}-.42 \\
=.78 \\
=1.17 \\
=.93 \\
-.32\end{array}$ \\
\hline $\begin{array}{lr} & 28.1 \\
\text { Oct. } & 29.9 \\
& 30.9 \\
\text { Nov. } & 8.9 \\
& 9.9\end{array}$ & $\begin{array}{l}\mathrm{P} \\
\mathrm{Ry} \\
\mathrm{Ry} \\
\mathrm{P} \\
\mathrm{Ry}\end{array}$ & & $\begin{array}{l}\text { I } \\
\text { II } \\
\text { II } \\
\text { II } \\
\text { II }\end{array}$ & $\begin{array}{rrr}13 & 43 & 11.550 \\
13 & 9 & 20.595 \\
13 & 13 & 10.294 \\
13 & 58 & 30.133 \\
14 & 4 & 14.974\end{array}$ & $\begin{array}{l}+.010 \\
+.115 \\
+.064 \\
+.183 \\
+.214\end{array}$ & $\begin{array}{l}\mathrm{C} \\
\mathrm{C} \\
\mathrm{C} \\
\mathrm{C} \\
\mathrm{C}\end{array}$ & $\begin{array}{rrr}-14 & 25 & 55.08 \\
-5 & 5 & 50.95 \\
-5 & 26 & 7.98 \\
-10 & 5 & 41.54 \\
-10 & 41 & 51.02\end{array}$ & $\begin{array}{l}-.08 \\
-.05 \\
-.08 \\
-.34 \\
+.38\end{array}$ \\
\hline $\begin{array}{l}16.0 \\
17.0 \\
23.0 \\
24.0\end{array}$ & $\begin{array}{l}\mathrm{P} \\
\mathrm{Bn} \\
\mathrm{Ry} \\
\mathrm{Ry}\end{array}$ & $\mathrm{E}$ & $\begin{array}{l}\text { II } \\
\text { II } \\
\text { II } \\
\text { C }\end{array}$ & $\begin{array}{rrr}14 & 40 & 5.030 \\
14 & 46 & 13.341 \\
15 & 23 & 46.981 \\
15 & 30 & 9.289\end{array}$ & $\begin{array}{r}+.170 \\
+.121 \\
+.091 \\
+.139\end{array}$ & $\begin{array}{l}\mathrm{C} \\
\mathbf{C} \\
\mathbf{C} \\
\mathrm{C}\end{array}$ & $\begin{array}{rrr}-14 & 18 & 36.27 \\
-14 & 53 & 39.36 \\
-18 & 10 & 46.54 \\
-18 & 41 & 0.59\end{array}$ & $\begin{array}{l}+.23 \\
+.06 \\
+.26 \\
-1.99\end{array}$ \\
\hline \begin{tabular}{cc}
\multicolumn{2}{c}{1923} \\
Jan. & 12.1 \\
Feb. & 18.9 \\
& 22.9 \\
Mar. & 7.9 \\
& 8.9
\end{tabular} & $\begin{array}{l}\mathrm{Bn} \\
\mathrm{Ry} \\
\mathrm{Bn} \\
\mathrm{P} \\
\mathrm{Bn}\end{array}$ & W & $\begin{array}{l}\text { I } \\
\text { C } \\
\text { II } \\
\text { II } \\
\text { II }\end{array}$ & $\begin{array}{lll}20 & 52 & 28.319 \\
20 & 22 & 58.366 \\
20 & 38 & 56.295 \\
21 & 45 & 34.674 \\
21 & 51 & 16.658\end{array}$ & $\begin{array}{l}+.069 \\
+.286 \\
+.045 \\
+.154 \\
+.158\end{array}$ & $\begin{array}{l}\mathrm{C} \\
\mathrm{C} \\
\mathrm{C} \\
\mathrm{C} \\
\mathrm{C}\end{array}$ & $\begin{array}{rrr}-18 & 19 & 44.03 \\
-18 & 43 & 40.75 \\
-18 & 30 & 15.32 \\
-15 & 19 & 5.80 \\
-14 & 55 & 9.23\end{array}$ & $\begin{array}{l}-.13 \\
+.25 \\
-.32 \\
-.40 \\
+1.77\end{array}$ \\
\hline $\begin{array}{r}20.0 \\
27.0 \\
29.0 \\
\text { Apr. } 2.0 \\
12.0\end{array}$ & $\begin{array}{l}\mathrm{Ry} \\
\mathrm{Bn} \\
\mathrm{P} \\
\mathrm{Ry} \\
\mathrm{P}\end{array}$ & & $\begin{array}{l}\text { C } \\
\text { II } \\
\text { II } \\
\text { II } \\
\text { C }\end{array}$ & $\begin{array}{rrr}22 & 57 & 20.452 \\
23 & 42 & 26.915 \\
23 & 55 & 50.522 \\
0 & 23 & 25.667 \\
1 & 37 & 27.494\end{array}$ & $\begin{array}{l}+.082 \\
+.185 \\
+.102 \\
+.127 \\
+.024\end{array}$ & $\begin{array}{l}\mathrm{C} \\
\mathrm{C} \\
\mathrm{C} \\
\mathrm{C} \\
\mathrm{C}\end{array}$ & 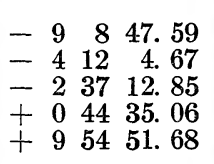 & $\begin{array}{r}-.99 \\
+1.23 \\
+.95 \\
+1.16 \\
-.42\end{array}$ \\
\hline $\begin{array}{rr} & 20.0 \\
& 27.0 \\
\text { May } & 1.1 \\
& 3.1 \\
4.1\end{array}$ & $\begin{array}{l}\mathrm{Bn} \\
\mathrm{Ry} \\
\mathrm{Ry} \\
\mathrm{P} \\
\mathrm{Bn}\end{array}$ & W & $\begin{array}{l}\mathrm{I} \\
\mathrm{I} \\
\mathrm{I} \\
\mathrm{I} \\
\mathrm{I}\end{array}$ & $\begin{array}{rrr}2 & 39 & 15 . \\
3 & 28 & 25.78 \\
3 & 51 & 55.083 \\
4 & 2 & 2.720 \\
4 & 6 & 40.217\end{array}$ & $\begin{array}{l}+.218 \\
+.054 \\
+.007 \\
+.050 \\
+.097\end{array}$ & $\begin{array}{l}\mathrm{C} \\
\mathrm{C} \\
\mathrm{C} \\
\mathrm{C} \\
\mathrm{C}\end{array}$ & $\begin{array}{rrr}+16 & 48 & 58.90 \\
+21 & 12 & 14.18 \\
+22 & 47 & 56.57 \\
+23 & 21 & 0.21 \\
+23 & 34 & 0.71\end{array}$ & $\begin{array}{r}-.70 \\
+.18 \\
+.87 \\
+.41 \\
+1.41\end{array}$ \\
\hline $\begin{array}{rr}\text { June } & 24.9 \\
& 25.9 \\
\text { July } & 5.0 \\
6.0 \\
23.0\end{array}$ & $\begin{array}{l}\text { Ry } \\
\text { Ry } \\
\text { Py } \\
\text { Ry }\end{array}$ & $\mathrm{E}$ & $\begin{array}{l}\text { II } \\
\text { II } \\
\text { II } \\
\text { II } \\
\text { C }\end{array}$ & $\begin{array}{rrr}4 & 39 & 0.755 \\
4 & 43 & 38.031 \\
5 & 38 & 1.089 \\
5 & 45 & 27 . \\
8 & 15 & 52.894 \\
\end{array}$ & $\begin{array}{l}+.085 \\
+.0151 \\
+.099 \\
+.054 \\
+.086\end{array}$ & $\begin{array}{l}\mathrm{C} \\
\mathrm{C} \\
\mathrm{C} \\
\mathrm{C} \\
\mathrm{C}\end{array}$ & 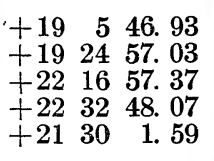 & $\begin{array}{l}+.33 \\
+.27 \\
+.07 \\
+.77 \\
+.49\end{array}$ \\
\hline $\begin{array}{ll} & 26.0 \\
\text { Aug. } & 13.1 \\
16.1 \\
21.1 \\
\text { Sept. } 10.1\end{array}$ & $\begin{array}{l}\mathrm{Bn} \\
\mathrm{Bn} \\
\mathrm{P} \\
\mathrm{P} \\
\mathrm{Ry}\end{array}$ & & $\begin{array}{l}\text { I } \\
\text { I } \\
\text { I } \\
\text { I } \\
\text { I }\end{array}$ & 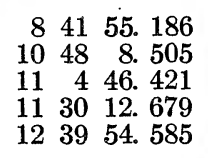 & $\begin{array}{l}+.266 \\
+.095 \\
+.001 \\
+.099 \\
+.015\end{array}$ & $\begin{array}{l}\mathrm{C} \\
\mathrm{C} \\
\mathrm{C} \\
\mathrm{C} \\
\mathrm{C}\end{array}$ & 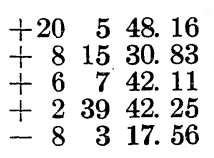 & $\begin{array}{l}+.86 \\
-1.97 \\
-1.49 \\
-.85 \\
+.04\end{array}$ \\
\hline $\begin{array}{rr} & 14.1 \\
\text { Oct. } & 8.0 \\
9.0 \\
10.9 \\
22.0\end{array}$ & $\begin{array}{l}\text { Ry } \\
\text { Ry } \\
P \\
P \\
\text { Ry }\end{array}$ & $\mathbf{E}$ & $\begin{array}{l}\text { I } \\
\text { II } \\
\text { II } \\
\text { II } \\
\text { II }\end{array}$ & $\begin{array}{rrr}12 & 44 & 16.411 \\
11 & 56 & 28.012 \\
11 & 57 & 30.208 \\
12 & 1 & 19.644 \\
12 & 50 & 39.176\end{array}$ & $\begin{array}{l}+.031 \\
+.052 \\
+.098 \\
+.064 \\
+.096\end{array}$ & $\begin{array}{l}\mathrm{C} \\
\mathrm{C} \\
\mathrm{C} \\
\mathrm{C} \\
\mathrm{C}\end{array}$ & 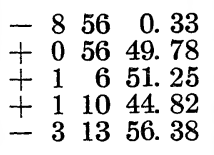 & $\begin{array}{r}-.53 \\
-.12 \\
+.05 \\
-.18 \\
-.28\end{array}$ \\
\hline
\end{tabular}




\begin{tabular}{|c|c|c|c|c|c|c|c|c|}
\hline \multicolumn{9}{|c|}{$\mathbf{M} \mathbf{E} \mathbf{C} \mathbf{U} \mathbf{Y}$} \\
\hline Date & Obsr. & $\mathrm{Cl}$. & Limb & $\begin{array}{l}\text { App. R. A. } \\
\text { of Center }\end{array}$ & $\begin{array}{c}\text { Corr. to Am. } \\
\text { Eph. }\end{array}$ & $\operatorname{Limb}$ & $\begin{array}{l}\text { App. Decl. } \\
\text { of Center }\end{array}$ & $\begin{array}{l}\text { Corr. to. } \\
\text { Am. Eph. }\end{array}$ \\
\hline 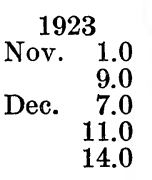 & $\begin{array}{l}\mathrm{P} \\
\mathrm{Bn} \\
\mathrm{P} \\
\mathrm{Bn} \\
\mathbf{P}\end{array}$ & W & $\begin{array}{l}\text { II } \\
\text { II } \\
\text { I } \\
\text { I } \\
\text { C }\end{array}$ & $\begin{array}{ccc}\mathrm{h} & \mathrm{m} & \mathrm{s} \\
13 & 50 & 49.966 \\
14 & 40 & 39.924 \\
17 & 44 & 33.270 \\
18 & 11 & 47.965 \\
18 & 31 & 59.225\end{array}$ & $\begin{aligned} & s \\
&-0.004 \\
&+.104 \\
&+.160 \\
&+.135 \\
&+.125\end{aligned}$ & $\begin{array}{l}\mathrm{C} \\
\mathbf{C} \\
\mathbf{C} \\
\mathbf{C} \\
\mathbf{C}\end{array}$ & \begin{tabular}{rrc}
$\circ$ & \multicolumn{1}{c}{} & \multicolumn{1}{c}{} \\
-9 & 56 & 47.21 \\
-15 & 5 & 36.22 \\
-25 & 29 & 50.50 \\
-25 & 39 & 40.88 \\
-25 & 31 & 33.18
\end{tabular} & $\begin{array}{r}\prime \prime \\
-0.11 \\
+.48 \\
-.80 \\
-.28 \\
-.58\end{array}$ \\
\hline 1924.0 & $\mathbf{P}$ & & I & $1851 \quad 40.940$ & +.120 & C & $\begin{array}{lll}-25 & 9 & 58.80\end{array}$ & -2.50 \\
\hline $\begin{array}{ll}\text { Jan. } & 27.9 \\
\text { Feb. } & 21.0 \\
& 28.0\end{array}$ & $\begin{array}{l}\mathrm{Ry} \\
\mathrm{P}\end{array}$ & & $\begin{array}{l}\text { II } \\
\text { II } \\
\text { II }\end{array}$ & $\begin{array}{rrr}18 & 59 & 18.394 \\
20 & 52 & 51.159 \\
21 & 36 & 0.842\end{array}$ & $\begin{array}{l}+.114 \\
+.129 \\
+.082\end{array}$ & $\begin{array}{l}\mathrm{C} \\
\mathrm{C}\end{array}$ & $\begin{array}{rrr}-20 & 34 & 59.70 \\
-19 & 1 & 44.01 \\
-16 & 22 & 12.74\end{array}$ & $\begin{array}{l}-.50 \\
+.19 \\
-.44\end{array}$ \\
\hline 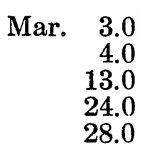 & $\begin{array}{l}\mathrm{Bn} \\
\mathrm{Bn} \\
\mathrm{P} \\
\mathrm{Ry} \\
\mathrm{Bn}\end{array}$ & W & $\begin{array}{l}\text { II } \\
\text { II } \\
\text { II } \\
\text { I } \\
\text { I }\end{array}$ & $\begin{array}{rrr}22 & 1 & 26.856 \\
22 & 7 & 53.105 \\
23 & 7 & 16.459 \\
0 & 24 & 13.993 \\
0 & 53 & 20.950\end{array}$ & $\begin{array}{l}+.106 \\
+.185 \\
+.139 \\
+.233 \\
+.140\end{array}$ & $\begin{array}{l}\mathrm{C} \\
\mathrm{C} \\
\mathrm{C} \\
\mathrm{C} \\
\mathrm{C}\end{array}$ & 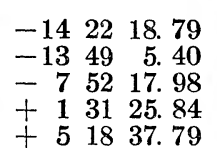 & $\begin{array}{l}-.09 \\
-.50 \\
+.22 \\
+.54 \\
-.81\end{array}$ \\
\hline $\begin{array}{lr}\text { Apr. } & 3.0 \\
8.0 \\
\\
15.1 \\
\text { May } 25.9 \\
\text { June } 18.9\end{array}$ & $\begin{array}{l}\mathrm{P} \\
\mathrm{Ry} \\
\mathrm{Bn} \\
\mathrm{Bn} \\
\mathrm{Bn}\end{array}$ & $\stackrel{\mathrm{E}}{\mathrm{W}}$ & $\begin{array}{l}\text { I } \\
\text { I } \\
\text { I } \\
\text { II } \\
\text { II }\end{array}$ & $\begin{array}{rrr}1 & 36 & 40.534 \\
2 & 10 & 3.105 \\
2 & 47 & 20.739 \\
2 & 47 & 44.627 \\
4 & 36 & 38.810\end{array}$ & $\begin{array}{l}+.054 \\
+.0125 \\
+.071 \\
+.077 \\
+.180\end{array}$ & $\begin{array}{l}\mathrm{C} \\
\mathrm{C} \\
\mathrm{C} \\
\mathrm{C} \\
\mathrm{C}\end{array}$ & 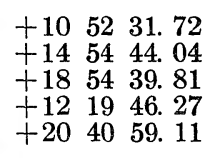 & $\begin{array}{l}+1.92 \\
+.54 \\
+1.51 \\
+.97 \\
-.69\end{array}$ \\
\hline $\begin{array}{ll} & 20.0 \\
& 30.0 \\
\text { July } & 11.0 \\
15.0 \\
18.0\end{array}$ & $\begin{array}{l}\text { Bn } \\
\text { Ry } \\
\text { Ry } \\
\text { Ry } \\
\text { Bn }\end{array}$ & & $\begin{array}{l}\text { II } \\
\text { II } \\
\text { I } \\
\text { I } \\
\text { I }\end{array}$ & $\begin{array}{rrr}4 & 44 & 7.067 \\
6 & 10 & 41.628 \\
7 & 53 & 53.504 \\
8 & 27 & 48.272 \\
8 & 51 & 19.829\end{array}$ & $\begin{array}{l}+.147 \\
+.138 \\
+.274 \\
+.222 \\
+.269\end{array}$ & $\begin{array}{l}\mathrm{C} \\
\mathrm{C} \\
\mathrm{C} \\
\mathrm{C} \\
\mathrm{C}\end{array}$ & $\begin{array}{rrr}+21 & 6 & 53.73 \\
+24 & 6 & 34.14 \\
+22 & 41 & 18.00 \\
+20 & 59 & 30.21 \\
+19 & 26 & 32.84\end{array}$ & $\begin{array}{l}-.57 \\
=.46 \\
-.70 \\
+.01 \\
-.76\end{array}$ \\
\hline $\begin{array}{ll} & 24.1 \\
29.1 \\
\text { Aug. } & 14.1 \\
15.1 \\
19.1\end{array}$ & $\begin{array}{l}\mathrm{Bn} \\
\mathrm{Bn} \\
\mathrm{P} \\
\mathrm{Ry} \\
\mathrm{P}\end{array}$ & $\underset{\mathrm{E}}{\mathrm{W}}$ & $\begin{array}{l}\text { I } \\
\text { I } \\
\text { I } \\
\text { I } \\
\text { I }\end{array}$ & $\begin{array}{rrr}9 & 33 & 30.653 \\
10 & 4 & 4.406 \\
11 & 16 & 53.176 \\
11 & 20 & 7.375 \\
11 & 31 & 11.949\end{array}$ & $\begin{array}{l}+.223 \\
+.246 \\
+.026 \\
+.025 \\
-.001\end{array}$ & $\begin{array}{l}\mathrm{C} \\
\mathrm{C} \\
\mathrm{C} \\
\mathrm{C} \\
\mathrm{C}\end{array}$ & $\begin{array}{r}+155257.90 \\
+123950.73 \\
+24159.85 \\
+29 \\
+\quad 01250.31 \\
+\quad 012\end{array}$ & $\begin{array}{l}-.90 \\
-2.77 \\
-1.05 \\
-.19 \\
+.53\end{array}$ \\
\hline $\begin{array}{r}\text { Oct. } \quad 2.0 \\
3.0 \\
9.0 \\
10.0 \\
13.0\end{array}$ & $\begin{array}{l}\mathrm{P} \\
\mathrm{Ry} \\
\mathrm{Bn} \\
\mathrm{P} \\
\mathrm{P}\end{array}$ & $\frac{\mathrm{E}}{\mathrm{W}}$ & $\begin{array}{l}\text { II } \\
\text { II } \\
\text { II } \\
\text { II } \\
\text { II }\end{array}$ & $\begin{array}{rrr}11 & 36 & 25.340 \\
11 & 42 & 6.057 \\
12 & 18 & 39 . \\
12 & 24 & 57 . \\
12 & 531 \\
12 & 43 & 55.492\end{array}$ & $\begin{array}{l}+.130 \\
+.087 \\
+.122 \\
+.111 \\
+.082\end{array}$ & $\begin{array}{l}\mathrm{C} \\
\mathrm{C} \\
\mathrm{C} \\
\mathrm{C} \\
\mathrm{C}\end{array}$ & 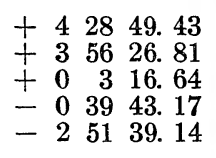 & $\begin{array}{l}+.13 \\
+.11 \\
+.74 \\
-.97 \\
-1.34\end{array}$ \\
\hline $\begin{array}{r}14.0 \\
16.0 \\
17.0 \\
20.0 \\
\text { Nov. } 17.0\end{array}$ & $\begin{array}{l}\mathrm{Bn} \\
\mathrm{Bn} \\
\mathrm{P} \\
\mathrm{P} \\
\mathrm{Ry}\end{array}$ & $\underset{\mathrm{E}}{\mathrm{W}}$ & $\begin{array}{l}\text { II } \\
\text { II } \\
\text { II } \\
\text { C } \\
\text { I }\end{array}$ & $\begin{array}{rrr}12 & 50 & 14.999 \\
13 & 2 & 52.583 \\
13 & 9 & 10.157 \\
13 . & 27 & 58.547 \\
16 & 23 & 30.067\end{array}$ & $\begin{array}{l}+.099 \\
+.183 \\
+.067 \\
+.177 \\
+.217\end{array}$ & $\begin{array}{l}\mathrm{C} \\
\mathrm{C} \\
\mathrm{C} \\
\mathrm{C} \\
\mathrm{C}\end{array}$ & $\begin{array}{lrrr}- & 3 & 36 & 7.37 \\
-5 & 5 & 8.80 \\
-5 & 49 & 30.03 \\
-\quad 8 & 0 & 50.77 \\
-23 & 24 & 17.64\end{array}$ & $\begin{array}{l}-.27 \\
-.40 \\
-1.53 \\
-1.07 \\
+.06\end{array}$ \\
\hline $\begin{array}{r}20.0 \\
24.0 \\
\text { Dec. } 2.1 \\
9.1\end{array}$ & $\begin{array}{l}\mathrm{P} \\
\mathrm{Bn} \\
\mathrm{Ry} \\
\mathrm{Bn}\end{array}$ & $\mathbf{E}$ & $\begin{array}{l}I \\
I \\
I \\
I\end{array}$ & $\begin{array}{rrr}16 & 42 & 50.402 \\
17 & 8 & 38.212 \\
17 & 58 & 41.129 \\
18 & 36 & 5.904\end{array}$ & $\begin{array}{l}+.092 \\
+.192 \\
+.219 \\
+.064\end{array}$ & $\begin{array}{l}\mathrm{C} \\
\mathrm{C} \\
\mathrm{C} \\
\mathrm{C}\end{array}$ & $\begin{array}{rrr}-24 & 17 & 20.71 \\
-25 & 10 & 20.04 \\
-25 & 50 & 44.89 \\
-25 & 12 & 0.69\end{array}$ & $\begin{array}{r}-2.91 \\
-1.14 \\
-.09 \\
-.89\end{array}$ \\
\hline \begin{tabular}{lr}
\multicolumn{2}{c}{1925} \\
Jan. & 5.4 \\
& 22.4 \\
Feb. & 5.5
\end{tabular} & $\begin{array}{l}\mathrm{Bn} \\
\mathrm{P} \\
\mathrm{P}\end{array}$ & W & $\begin{array}{l}\text { II } \\
\text { II } \\
\text { II }\end{array}$ & $\begin{array}{lll}17 & 46 & 45.345 \\
18 & 36 & 58.049 \\
19 & 59 & 19.754\end{array}$ & $\begin{array}{l}+.005 \\
+.159 \\
+.154\end{array}$ & $\stackrel{\mathrm{C}}{\mathrm{C}}$ & $\begin{array}{llll}-20 & 18 & \\
-22 & 31 & 38.98 \\
-21 & 48 & 29.38\end{array}$ & $\begin{array}{l}-.88 \\
-.58\end{array}$ \\
\hline
\end{tabular}




\begin{tabular}{|c|c|c|c|c|c|c|c|c|}
\hline \multicolumn{9}{|c|}{$\mathbf{V} \mathbf{E} \mathbf{N} \mathbf{S}$} \\
\hline Date & Obsr. & Cl. & Limb & $\begin{array}{l}\text { App. R. A. } \\
\text { of Center }\end{array}$ & $\begin{array}{l}\text { Corr. to } \\
\text { Am. Eph. }\end{array}$ & Limb & $\begin{array}{l}\text { App. Decl. } \\
\text { of Center }\end{array}$ & $\begin{array}{l}\text { Corr. to } \\
\text { Am. Eph. }\end{array}$ \\
\hline \begin{tabular}{cc}
\multicolumn{2}{c}{1919} \\
June 3.1 \\
13.1 \\
16.1 \\
19.1 \\
20.1
\end{tabular} & $\begin{array}{l}\mathrm{M} \\
\mathrm{P} \\
\mathrm{Sr} \\
\mathrm{Sr} \\
\mathrm{Sr}\end{array}$ & W & $\begin{array}{l}\text { I } \\
\text { I } \\
\text { I } \\
\text { I }\end{array}$ & 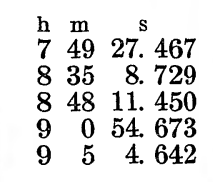 & $\begin{array}{l}\text { s } \\
+0.127 \\
+.019 \\
+.050 \\
=.017 \\
-.008\end{array}$ & $\begin{array}{l}\mathbf{N} \\
\mathbf{N} \\
\mathbf{N} \\
\mathbf{N} \\
\mathbf{N}\end{array}$ & \begin{tabular}{rrr} 
& \multicolumn{1}{c}{${ }^{\prime \prime}$} \\
+23 & 28 & 58.82 \\
+20 & 57 & 2.26 \\
+20 & 2 & 9.14 \\
+19 & 3 & 34.25 \\
+18 & 43 & 16.74
\end{tabular} & $\begin{aligned} & 11 \\
&-1.18 \\
&=.54 \\
&-1.26 \\
&-1.25 \\
&-1.06\end{aligned}$ \\
\hline $\begin{array}{cr} & 23.1 \\
& 30.1 \\
\text { July } & 1.1 \\
& 3.1 \\
\text { Aug. } & 8.1\end{array}$ & $\begin{array}{l}\mathrm{Sr} \\
\mathrm{Sr} \\
\mathrm{P} \\
\mathrm{P} \\
\mathrm{Sr}\end{array}$ & & $\begin{array}{l}\text { I } \\
\text { I } \\
\text { I } \\
\text { I }\end{array}$ & $\begin{array}{rrrr}9 & 17 & 20.946 \\
9 & 44 & 37.104 \\
9 & 48 & 21.233 \\
9 & 55 & 41.829 \\
11 & 31 & 47.443\end{array}$ & $\begin{array}{l}+.016 \\
\pm .066 \\
+.003 \\
\pm .039 \\
-.067\end{array}$ & $\begin{array}{l}\mathbf{N} \\
\mathbf{N} \\
\mathbf{N} \\
\mathbf{N} \\
\mathbf{N}\end{array}$ & 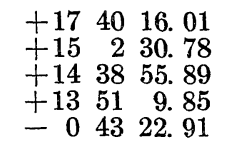 & $\begin{array}{l}=.89 \\
=.02 \\
=.91 \\
=.75 \\
-1.41\end{array}$ \\
\hline $\begin{array}{l}11.1 \\
18.1 \\
\text { Sept. } 15.0 \\
18.0 \\
24.9\end{array}$ & $\begin{array}{l}\mathrm{M} \\
\mathrm{M} \\
\mathrm{P} \\
\mathrm{P} \\
\mathrm{M}\end{array}$ & W & $\begin{array}{l}\text { I } \\
\text { I } \\
\text { II } \\
\text { II } \\
\text { II }\end{array}$ & $\begin{array}{rrr}11 & 35 & 29.466 \\
11 & 40 & 10.571 \\
11 & 1 & 13.506 \\
10 & 55 & 9.507 \\
10 & 44 & 44.017\end{array}$ & $\begin{array}{l} \pm .006 \\
\pm .019 \\
=.124 \\
=.113 \\
-.003\end{array}$ & $\begin{array}{l}\mathrm{N} \\
\mathrm{N} \\
\mathrm{N}\end{array}$ & 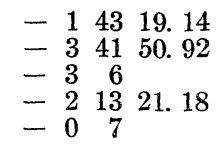 & $\begin{array}{l}=.74 \\
-.72 \\
+.72\end{array}$ \\
\hline $\begin{array}{r}\text { Oct. } \quad 6.9 \\
9.9 \\
12.9 \\
19.9 \\
. \quad 27.9\end{array}$ & $\begin{array}{l}\mathrm{M} \\
\mathrm{M} \\
\mathrm{P} \\
\mathrm{Sr} \\
\mathrm{M}\end{array}$ & $\mathrm{E}$ & $\begin{array}{l}\text { II } \\
\text { II } \\
\text { II } \\
\text { II } \\
\text { II }\end{array}$ & $\begin{array}{rrr}10 & 43 & 15.899 \\
10 & 46 & 9.043 \\
10 & 50 & 11.040 \\
11 & 3 & 26.6272 \\
11 & 23 & 46.502\end{array}$ & $\begin{array}{l}-.031 \\
=.067 \\
+.030 \\
+.062 \\
+.042\end{array}$ & $\begin{array}{l}\mathbf{S} \\
\mathbf{S} \\
\mathbf{S} \\
\mathbf{S} \\
\mathbf{S}\end{array}$ & $\begin{array}{rrr}+235 & 4.51 \\
+257 & 41.61 \\
+ & 312 & 21.73 \\
+316 & 40.24 \\
+ & 234 & 59.63\end{array}$ & $\begin{array}{l}+.21 \\
+.41 \\
\pm .37 \\
+.76 \\
+.13\end{array}$ \\
\hline Nov. $\begin{array}{r}5.9 \\
9.9 \\
13.9 \\
16.9 \\
17.9\end{array}$ & $\begin{array}{l}\mathrm{P} \\
\mathrm{P} \\
\mathrm{P} \\
\mathrm{Sr} \\
\mathrm{Sr}\end{array}$ & & $\begin{array}{l}\text { II } \\
\text { II } \\
\text { II } \\
\text { II } \\
\text { II }\end{array}$ & $\begin{array}{rrr}11 & 51 & 22.232 \\
12 & 4 & 50.411 \\
12 & 18 & 54.727 \\
12 & 29 & 48.638 \\
12 & 33 & 30.388\end{array}$ & $\begin{array}{l}+.062 \\
+.101 \\
+.147 \\
+.012 \\
+.088\end{array}$ & $\begin{array}{l}\mathbf{S} \\
\mathbf{S} \\
\mathbf{S} \\
\mathbf{S} \\
\mathbf{S}\end{array}$ & 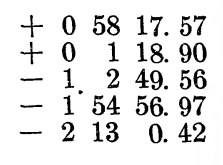 & $\begin{array}{l}-.53 \\
=.70 \\
-1.66 \\
=.57 \\
-.32\end{array}$ \\
\hline $\begin{array}{l}19.9 \\
20.9 \\
23.9 \\
24.9\end{array}$ & $\begin{array}{l}\mathrm{P} \\
\mathrm{Sr} \\
\mathrm{P} \\
\mathrm{P}\end{array}$ & & $\begin{array}{l}\text { II } \\
\text { II } \\
\text { II } \\
\text { II }\end{array}$ & $\begin{array}{rrr}12 & 40 & 58.833 \\
12 & 44 & 45.521 \\
12 & 56 & 15.193 \\
13 & 0 & 8.064\end{array}$ & $\begin{array}{l}+.063 \\
+.051 \\
+.153 \\
+.134\end{array}$ & $\begin{array}{l}\mathbf{S} \\
\mathbf{S} \\
\mathbf{S} \\
\mathbf{S}\end{array}$ & 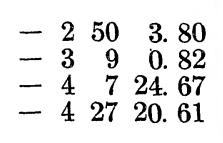 & $\begin{array}{l}-.70 \\
=.42 \\
=.97 \\
-1.81\end{array}$ \\
\hline \begin{tabular}{cr}
\multicolumn{2}{c}{1921} \\
Feb. & 4.1 \\
14.1 \\
15.1 \\
18.1 \\
28.1
\end{tabular} & $\begin{array}{l}\mathrm{Ry} \\
\mathrm{Bn} \\
\mathrm{Bn} \\
\mathrm{Ry} \\
\mathrm{Bn}\end{array}$ & & $\begin{array}{l}\text { I } \\
\text { I } \\
\text { I } \\
\text { I } \\
\text { I }\end{array}$ & $\begin{array}{rrr}0 & 6 & 55.792 \\
0 & 42 & 30.009 \\
0 & 45 & 55.131 \\
0 & 55 & 59.292 \\
1 & 27 & 20.190\end{array}$ & $\begin{array}{l}+.062 \\
\pm .081 \\
\pm .111 \\
-.028 \\
-.020\end{array}$ & $\begin{array}{l}\mathbf{S} \\
\mathbf{S} \\
\mathbf{S} \\
\mathbf{S} \\
\mathbf{S}\end{array}$ & $\begin{array}{r}+12827.37 \\
+62612.32 \\
+6557.80 \\
+820 \\
+1245.05 \\
+124748.81\end{array}$ & $\begin{array}{l}+.77 \\
\pm .58 \\
+.20 \\
+.15 \\
+.61\end{array}$ \\
\hline $\begin{array}{rr}\text { Mar. } & 1.1 \\
10.1 \\
11.1 \\
15.1 \\
17.1\end{array}$ & $\begin{array}{l}\mathrm{Bn} \\
\mathrm{P} \\
\mathrm{Bn} \\
\mathrm{Bn} \\
\mathrm{P}\end{array}$ & & $\begin{array}{l}\text { I } \\
\text { I } \\
\text { I } \\
\text { I } \\
\text { I }\end{array}$ & $\begin{array}{rrr}1 & 30 & 15.030 \\
1 & 54 & 4.220 \\
1 & 56 & 24.245 \\
2 & 4 & 57.229 \\
2 & 8 & 42.438\end{array}$ & $\begin{array}{l}+.050 \\
\pm .070 \\
+.005 \\
\pm .051 \\
-.032\end{array}$ & $\begin{array}{l}\underset{S}{S} \\
\mathbb{S} \\
\text { S } \\
\mathbf{S}\end{array}$ & 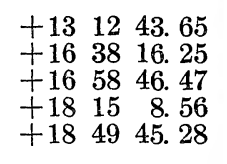 & $\begin{array}{l}+.35 \\
\pm .25 \\
\pm 1.07 \\
-.94 \\
-.02\end{array}$ \\
\hline $\begin{array}{l}18.1 \\
21.1 \\
25.1\end{array}$ & $\begin{array}{l}\mathrm{Ry} \\
\mathrm{Ry} \\
\mathrm{Bn}\end{array}$ & $\mathrm{E}$ & $\begin{array}{l}\text { I } \\
\text { I } \\
\text { I }\end{array}$ & $\begin{array}{rrr}2 & 10 & 26.222 \\
2 & 15 & 0.252 \\
2 & 19 & 27.666\end{array}$ & $\begin{array}{l}=.168 \\
=.058 \\
=.074\end{array}$ & $\stackrel{\mathrm{S}}{\mathrm{S}}$ & $\begin{array}{rrr}+1963.11 \\
+1950 & 38.20 \\
+2039\end{array}$ & $\begin{array}{l}-1.29 \\
+.30\end{array}$ \\
\hline $\begin{array}{ll}\text { Apr. } & 11.0 \\
& 12.0\end{array}$ & $\begin{array}{l}\mathrm{Bn} \\
\mathrm{Bn}\end{array}$ & $\tilde{W}$ & $\begin{array}{l}\mathrm{I} \\
\mathrm{I}\end{array}$ & $\begin{array}{lll}2 & 13 & 13.231 \\
2 & 11 & 34.328\end{array}$ & $\begin{array}{l}-.099 \\
-.092\end{array}$ & $\stackrel{\mathrm{S}}{\mathrm{S}}$ & $\begin{array}{rrr}+20 & 57 & 4.84 \\
+20 & 46 & 19.36\end{array}$ & $\begin{array}{l}-1.46 \\
-.94\end{array}$ \\
\hline $\begin{array}{r}15.0 \\
19.0 \\
\text { May } 8.9 \\
9.9 \\
16.9\end{array}$ & $\begin{array}{l}\text { Ry } \\
\text { Ry } \\
\text { Bn } \\
\text { Bn } \\
\text { Ry }\end{array}$ & & $\begin{array}{l}\text { I } \\
\text { I } \\
\text { II } \\
\text { II } \\
\text { II }\end{array}$ & $\begin{array}{rrr}2 & 5 & 59.118 \\
1 & 57 & 29.397 \\
1 & 26 & 31.104 \\
1 & 26 & 16.822 \\
1 & 28 & 34.737\end{array}$ & $\begin{array}{l}=.182 \\
\bar{x} .143 \\
\pm .014 \\
-.088 \\
-.033\end{array}$ & $\begin{array}{l}\mathrm{S} \\
\mathbf{S} \\
\mathrm{N} \\
\mathrm{N} \\
\mathrm{N}\end{array}$ & 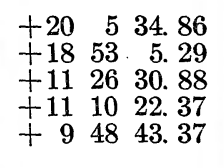 & $\begin{array}{l}-1.34 \\
-2.21 \\
=.92 \\
+.33 \\
+.17\end{array}$ \\
\hline $\begin{array}{r}18.9 \\
19.9 \\
30.9 \\
\text { June } \\
2.9 \\
5.9\end{array}$ & $\begin{array}{l}\mathrm{P} \\
\mathrm{Bn} \\
\mathrm{Ry} \\
\mathrm{Bn} \\
\mathrm{Bn}\end{array}$ & $\mathrm{W}$ & $\begin{array}{l}\text { II } \\
\text { II } \\
\text { II } \\
\text { II } \\
\text { II }\end{array}$ & $\begin{array}{rrr}1 & 30 & 25.522 \\
1 & 31 & 31.849 \\
1 & 50 & 36.241 \\
1 & 57 & 39.061 \\
2 & 5 & 20.285\end{array}$ & $\begin{array}{l}+.008 \\
+.019 \\
+.051 \\
+.001 \\
+.025\end{array}$ & $\begin{array}{l}N \\
N \\
N \\
N \\
N\end{array}$ & $\begin{array}{r}+93526.59 \\
+93024.88 \\
+93712.90 \\
+95532.22 \\
+101916.38\end{array}$ & $\begin{array}{l}\mp .01 \\
+.08 \\
.30 \\
+.02 \\
-.12\end{array}$ \\
\hline
\end{tabular}




\begin{tabular}{|c|c|c|c|c|c|c|c|c|}
\hline \multicolumn{9}{|c|}{$\mathbf{V} \mathbf{E} \mathbf{N} \mathbf{S}$} \\
\hline Date & Obsr. & Cl. & $\operatorname{Limb}$ & $\begin{array}{l}\text { App. R. A. } \\
\text { of Center }\end{array}$ & $\begin{array}{l}\text { Corr. to } \\
\text { Am. Eph. }\end{array}$ & Limb & $\begin{array}{l}\text { App. Decl. } \\
\text { of Center }\end{array}$ & $\begin{array}{l}\text { Corr. to } \\
\text { Am. Eph. }\end{array}$ \\
\hline $\begin{array}{r}1921 \\
\text { June } 12.9 \\
19.9 \\
23.9 \\
26.9 \\
27.9\end{array}$ & $\begin{array}{l}\text { Ry } \\
\mathbf{P} \\
\text { Ry } \\
\text { Ry } \\
\text { Ry }\end{array}$ & W & $\begin{array}{l}\text { II } \\
\text { II } \\
\text { II } \\
\text { II } \\
\text { II }\end{array}$ & \begin{tabular}{rrr}
$\mathrm{h}$ & $\mathrm{m}$ & \multicolumn{1}{c}{$\mathrm{s}$} \\
2 & 25 & 24.683 \\
2 & 48 & 1.862 \\
3 & 1 & 56.619 \\
3 & 12 & 48.434 \\
3 & 16 & 30.350
\end{tabular} & $\begin{array}{r}\text { s } \\
-0.067 \\
+\quad .082 \\
+\quad .059 \\
+.074 \\
+.070\end{array}$ & $\begin{array}{l}\mathbf{N} \\
\mathbf{N} \\
\mathbf{N} \\
\mathbf{N} \\
\mathbf{N}\end{array}$ & $\begin{array}{rcc}\circ & \prime & \prime \prime \\
+11 & 31 & 16.79 \\
+12 & 59 & 21.09 \\
+13 & 53 & 50.95 \\
+14 & 35 & 38.94 \\
+14 & 49 & 39.76\end{array}$ & $\begin{aligned} & \prime \prime \\
&-0.21 \\
&-.61 \\
&+.45 \\
&+.06 \\
&+.16\end{aligned}$ \\
\hline $\begin{array}{r}\text { July } \quad 17.9 \\
19.9\end{array}$ & $\begin{array}{l}\mathrm{Ry} \\
\mathrm{Bn}\end{array}$ & $\mathrm{W}$ & $\begin{array}{l}\text { II } \\
\text { II }\end{array}$ & $\begin{array}{lll}4 & 37 & 34.066 \\
4 & 46 & 20.071\end{array}$ & $\begin{array}{l}+.066 \\
+.051\end{array}$ & $\stackrel{N}{N}$ & $\begin{array}{rrr}+19 & 8 & 0.73 \\
+19 & 28 & 53.33\end{array}$ & $\begin{array}{l}+.23 \\
-.57\end{array}$ \\
\hline \begin{tabular}{ll}
\multicolumn{2}{c}{1922} \\
Sept. & 5.1 \\
& 7.1
\end{tabular} & $\begin{array}{l}\mathrm{Bn} \\
\mathrm{Bn}\end{array}$ & $\mathrm{E}$ & $\begin{array}{l}\text { I } \\
\text { I }\end{array}$ & $\begin{array}{lll}13 & 42 & 55.217 \\
13 & 50 & 30.069\end{array}$ & $\begin{array}{l}+.067 \\
+.079\end{array}$ & $\stackrel{N}{N}$ & $\begin{array}{lll}-12 & 50 & 13.40 \\
-13 & 44 & 16.34\end{array}$ & $\begin{array}{l}-.30 \\
-1.64\end{array}$ \\
\hline $\begin{array}{l}14.1 \\
15.1 \\
25.1 \\
26.1 \\
28.1\end{array}$ & $\begin{array}{l}\mathrm{P} \\
\mathrm{Ry} \\
\mathrm{Ry} \\
\mathrm{P} \\
\mathrm{P}\end{array}$ & & $\begin{array}{l}\text { I } \\
\text { I } \\
\text { I } \\
\text { I } \\
\text { I }\end{array}$ & $\begin{array}{rrr}14 & 16 & 46.984 \\
14 & 20 & 29.949 \\
14 & 56 & 52.806 \\
15 & 0 & 24.841 \\
15 & 7 & 23.978\end{array}$ & $\begin{array}{l}+.144 \\
+.119 \\
.014 \\
+.091 \\
+.128\end{array}$ & $\begin{array}{l}\mathbf{N} \\
\mathbf{N} \\
\mathbf{N} \\
\mathbf{N} \\
\mathbf{N}\end{array}$ & $\begin{array}{rrr}-16 & 44 & 16.05 \\
-17 & 8 & 43.13 \\
-20 & 52 & 46.50 \\
-21 & 12 & 58.60 \\
-21 & 52 & 2.06\end{array}$ & $\begin{array}{r}-.35 \\
-.83 \\
+.50 \\
-.40 \\
-.66\end{array}$ \\
\hline $\begin{array}{lr}\text { Oct. } & 3.1 \\
& 5.1 \\
12.1 \\
13.1 \\
\text { Nov. } 10.0\end{array}$ & $\begin{array}{l}\text { Ry } \\
P \\
P \\
\text { Ry } \\
\text { Ry }\end{array}$ & & $\begin{array}{l}\text { I } \\
\text { I } \\
\text { I } \\
\text { I } \\
\text { I }\end{array}$ & $\begin{array}{rrr}15 & 24 & 18.208 \\
15 & 30 & 47.644 \\
15 & 51 & 53.194 \\
15 & 54 & 38.964 \\
16 & 26 & 6.177\end{array}$ & $\begin{array}{l}+.078 \\
+.084 \\
-.016 \\
-.176 \\
-.273\end{array}$ & $\begin{array}{l}N \\
N \\
N \\
N \\
N\end{array}$ & $\begin{array}{rrr}-23 & 21 & 36.28 \\
-23 & 54 & 4.20 \\
-25 & 31 & 24.07 \\
-25 & 43 & 9.03 \\
-26 & 44 & 48.66\end{array}$ & $\begin{array}{l}-.88 \\
-1.80 \\
-.17 \\
+.57 \\
+1.54\end{array}$ \\
\hline $\begin{array}{r}13.0 \\
14.0 \\
16.0 \\
\text { Dec. } 17.9\end{array}$ & $\begin{array}{l}\text { Ry } \\
\text { Ry } \\
\text { P } \\
\text { Bn }\end{array}$ & $\mathrm{E}$ & $\begin{array}{l}\text { I } \\
\text { I } \\
\text { I } \\
\text { II }\end{array}$ & $\begin{array}{lll}16 & 22 & 22.940 \\
16 & 20 & 50.404 \\
16 & 17 & 21.044 \\
15 & 32 & 15.194\end{array}$ & $\begin{array}{l}-.140 \\
-.156 \\
-.286 \\
+.104\end{array}$ & $\begin{array}{l}N \\
N \\
N \\
S\end{array}$ & $\begin{array}{rrr}-26 & 11 & 59.53 \\
-25 & 58 & 56.97 \\
-25 & 29 & 49.58 \\
-16 & 4 & 27.61\end{array}$ & $\begin{array}{r}+.67 \\
+1.53 \\
+.32 \\
+.49\end{array}$ \\
\hline \begin{tabular}{cc}
\multicolumn{2}{c}{1923} \\
Jan. & 10.9 \\
11.9 \\
28.9 \\
29.9 \\
Feb. 18.9
\end{tabular} & $\begin{array}{l}\mathrm{P} \\
\mathrm{Bn} \\
\mathrm{Bn} \\
\mathrm{Bn} \\
\mathrm{Ry}\end{array}$ & $\mathrm{W}$ & $\begin{array}{l}\text { II } \\
\text { II } \\
\text { II } \\
\text { II } \\
\text { II }\end{array}$ & $\begin{array}{rrr}16 & 20 & 36.789 \\
16 & 23 & 45.961 \\
17 & 26 & 8.488 \\
17 & 30 & 14.192 \\
18 & 58 & 36.732\end{array}$ & $\begin{array}{l}+.069 \\
+.081 \\
+.078 \\
+.122 \\
+.122\end{array}$ & $\begin{array}{l}\mathrm{S} \\
\mathbf{S} \\
\\
\mathrm{S} \\
\mathrm{N}\end{array}$ & $\begin{array}{rrr}-17 & 0 & 47.45 \\
-17 & 8 & 33.44 \\
-19 & 20 & \\
-19 & 25 & 51.08 \\
-20 & 14 & 17.81\end{array}$ & $\begin{array}{l}-.55 \\
-1.04 \\
-.38 \\
+1.09\end{array}$ \\
\hline $\begin{array}{r}22.9 \\
28.9 \\
\text { Mar. } 1.9 \\
7.9 \\
8.9\end{array}$ & $\begin{array}{l}\mathrm{Bn} \\
\mathbf{P} \\
\mathrm{Ry} \\
\mathrm{P} \\
\mathrm{Bn}\end{array}$ & & $\begin{array}{l}\text { II } \\
\text { II } \\
\text { II } \\
\text { II } \\
\text { II }\end{array}$ & $\begin{array}{lll}19 & 17 & 16.346 \\
19 & 45 & 32.311 \\
19 & 50 & 16.188 \\
20 & 18 & 42.276 \\
20 & 23 & 26.659\end{array}$ & $\begin{array}{l}+.156 \\
+.131 \\
+.118 \\
+.116 \\
+.149\end{array}$ & $\begin{array}{l}\mathbf{N} \\
\mathbf{N} \\
\mathbf{N} \\
\mathbf{N} \\
\mathbf{N}\end{array}$ & $\begin{array}{rrr}-20 & 1 & 54.78 \\
-19 & 27 & 38.27 \\
-19 & 20 & 3.36 \\
-18 & 23 & 34.30 \\
-18 & 12 & 20.95\end{array}$ & $\begin{array}{l}-.38 \\
-.37 \\
+.64 \\
+1.20 \\
+.05\end{array}$ \\
\hline $\begin{array}{r}14.9 \\
25.9 \\
26.9 \\
28.9 \\
\text { Apr. } \quad 1.9\end{array}$ & $\begin{array}{l}\mathrm{P} \\
\mathrm{Bn} \\
\mathrm{Bn} \\
\mathrm{P} \\
\mathrm{Ry}\end{array}$ & W & $\begin{array}{l}\text { II } \\
\text { II } \\
\text { II } \\
\text { II } \\
\text { II }\end{array}$ & $\begin{array}{rrr}20 & 51 & 48.613 \\
21 & 43 & 12.126 \\
21 & 47 & 49.292 \\
21 & 57 & 1.955 \\
22 & 15 & 20.553\end{array}$ & $\begin{array}{l}+.063 \\
+.146 \\
+.142 \\
+.145 \\
+.123\end{array}$ & $\begin{array}{l}\mathrm{N} \\
\mathrm{N} \\
\mathrm{N} \\
\mathrm{N} \\
\mathrm{N}\end{array}$ & $\begin{array}{rrr}-16 & 54 & 15.80 \\
-13 & 47 & 9.79 \\
-13 & 27 & 34.67 \\
-12 & 47 & 14.75 \\
-11 & 22 & 14.42\end{array}$ & $\begin{array}{l}+.70 \\
\pm .99 \\
-.27 \\
+1.25 \\
+.48\end{array}$ \\
\hline \begin{tabular}{rr}
\multicolumn{2}{c}{1924} \\
Apr. & 3.1 \\
8.1 \\
14.1 \\
15.1
\end{tabular} & $\begin{array}{l}\mathrm{P} \\
\mathrm{Ry} \\
\mathrm{Bn} \\
\mathrm{Bn}\end{array}$ & $\mathrm{E}$ & $\begin{array}{l}\text { I } \\
\text { I } \\
\text { I } \\
\text { I }\end{array}$ & 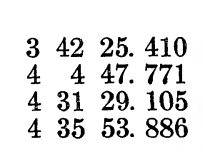 & $\begin{array}{r}-.060 \\
+.061 \\
+.025 \\
-.044\end{array}$ & $\begin{array}{l}\mathbf{S} \\
\mathbf{S} \\
\mathbf{S} \\
\mathbf{S}\end{array}$ & 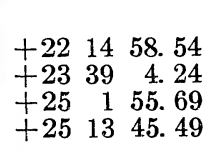 & $\begin{array}{l}-1.26 \\
-.56 \\
+.39 \\
+.49\end{array}$ \\
\hline $\begin{array}{rr} & 24.1 \\
& 25.1 \\
\text { May } & 1.1 \\
6.1 \\
22.1\end{array}$ & $\begin{array}{l}\mathrm{P} \\
\mathrm{Bn} \\
\mathrm{P} \\
\mathrm{Ry} \\
\mathrm{P}\end{array}$ & & $\begin{array}{l}\text { I } \\
\text { I } \\
\text { I } \\
\text { I } \\
\text { I }\end{array}$ & $\begin{array}{rrr}5 & 14 & 49.856 \\
5 & 19 & 2.433 \\
5 & 43 & 35.148 \\
6 & 2 & 52.617 \\
6 & 53 & 28.647\end{array}$ & $\begin{array}{l}-.124 \\
-.017 \\
+.018 \\
-.093 \\
-.063\end{array}$ & $\begin{array}{l}\mathbf{S} \\
\mathbf{S} \\
\mathbf{S} \\
\mathbf{S} \\
\mathbf{N}\end{array}$ & $\begin{array}{rrr}+26 & 34 & 17.81 \\
+26 & 40 & 22.32 \\
+27 & 5 & 5.21 \\
+27 & 10 & 53.11 \\
+26 & 12 & 40.50\end{array}$ & $\begin{array}{l}+.21 \\
+.12 \\
+1.01 \\
+1.01 \\
+1.00\end{array}$ \\
\hline $\begin{array}{cr} & 26.1 \\
\text { June } & 5.1 \\
& 26.0 \\
\text { July } & 10.0 \\
& 11.0\end{array}$ & $\begin{array}{l}\mathrm{Bn} \\
\mathrm{P} \\
\mathrm{Ry} \\
\mathrm{P} \\
\mathrm{Ry}\end{array}$ & $\begin{array}{l}\mathbf{E} \\
\mathrm{W} \\
\mathrm{W}\end{array}$ & $\begin{array}{l}\text { I } \\
\text { I } \\
\text { I } \\
\text { II }\end{array}$ & $\begin{array}{rrrr}7 & 2 & 26 . & 147 \\
7 & 15 & 51.285 \\
6 & 52 & 25.094 \\
6 & 17 & \\
6 & 15 & 19 . & 232\end{array}$ & $\begin{array}{l}-.043 \\
-.145 \\
-.096 \\
+.032\end{array}$ & $\begin{array}{l}\mathbf{N} \\
\mathbf{N} \\
\mathbf{N} \\
\mathbf{N} \\
\mathbf{N}\end{array}$ & $\begin{array}{rrr}+25 & 43 & 48.79 \\
+24 & 16 & 2.43 \\
+20 & 35 & 45.57 \\
+18 & 22 & 16.71 \\
+18 & 15 & 24.50\end{array}$ & $\begin{array}{l} \pm .59 \\
\pm .37 \\
\pm .07 \\
\pm .79 \\
-.30\end{array}$ \\
\hline
\end{tabular}


NINE-INCH TRANSIT CIRCLE OBSERVATIONS, 1913-1926

\begin{tabular}{|c|c|c|c|c|c|c|c|c|}
\hline \multicolumn{9}{|c|}{ VEN US } \\
\hline Date & Obsr. & $\mathrm{Cl}$. & $\operatorname{Limb}$ & $\begin{array}{l}\text { App. R. A. } \\
\text { of Center }\end{array}$ & $\begin{array}{l}\text { Corr. to } \\
\text { Am. Eph. }\end{array}$ & $\operatorname{Limb}$ & $\begin{array}{l}\text { App. Decl. } \\
\text { of Center }\end{array}$ & $\begin{array}{l}\text { Corr. to } \\
\text { Am. Eph. }\end{array}$ \\
\hline \begin{aligned} & \multicolumn{2}{c}{1924} \\
& July 13.9 \\
&. 14.9 \\
& 16.9 \\
& 23.9 \\
& 27.9\end{aligned} & $\begin{array}{l}\text { Ry } \\
R y \\
P \\
\text { Bn } \\
\text { Ry }\end{array}$ & W & $\begin{array}{l}\text { II } \\
\text { II } \\
\text { II } \\
\text { II } \\
\text { II }\end{array}$ & $\begin{array}{ccc}\mathrm{h} & \mathrm{m} & \mathrm{s} \\
6 & 10 & 24.729 \\
6 & 9 & 5.282 \\
6 & 6 & 55.472 \\
6 & 4 & 35.404 \\
6 & 6 & 45.248\end{array}$ & $\begin{aligned} & \mathrm{s} \\
&-0.051 \\
&+.042 \\
&-.038 \\
&+.054 \\
&+.028\end{aligned}$ & $\begin{array}{l}\mathbf{N} \\
\mathbf{N} \\
\mathbf{N} \\
\mathbf{N} \\
\mathbf{N}\end{array}$ & 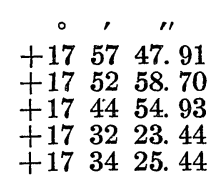 & $\begin{array}{l}\quad 0.69 \\
-0.69 \\
=.50 \\
-.37 \\
+.14 \\
-.06\end{array}$ \\
\hline $\begin{array}{lr} & 28.9 \\
\text { Aug. } & 4.9 \\
27.9 \\
28.9 \\
\text { Sept. } \quad 1.9\end{array}$ & $\begin{array}{l}\mathrm{Bn} \\
\mathrm{Bn} \\
\mathrm{P} \\
\mathrm{Ry} \\
\mathrm{P}\end{array}$ & $\begin{array}{l}\mathrm{W} \\
\mathrm{E}\end{array}$ & $\begin{array}{l}\text { II } \\
\text { II } \\
\text { II } \\
\text { II } \\
\text { II }\end{array}$ & $\begin{array}{rrr}6 & 7 & 39.733 \\
6 & 17 & 40.099 \\
7 & 23 & 9.525 \\
7 & 26 & 46.931 \\
7 & 41 & 42.141\end{array}$ & $\begin{array}{l}+.073 \\
\pm .021 \\
+.125 \\
+.141 \\
+.141\end{array}$ & $\begin{array}{l}\mathbf{N} \\
\mathbf{N} \\
\mathbf{S} \\
\mathbf{S} \\
\mathbf{S}\end{array}$ & $\begin{array}{rrr}+17 & 35 & 43.57 \\
+17 & 50 & 41.87 \\
+18 & 22 & 3.64 \\
+18 & 20 & 20.26 \\
+18 & 927.86\end{array}$ & $\begin{array}{l}-.33 \\
+.87 \\
-1.36 \\
+.04 \\
+.66\end{array}$ \\
\hline $\begin{array}{r}3.9 \\
10.9 \\
17.9 \\
\\
22.9 \\
\text { Oct. } 1.9\end{array}$ & $\begin{array}{l}\mathrm{P} \\
\mathrm{P} \\
\mathrm{P} \\
\mathrm{Ry} \\
\mathrm{P}\end{array}$ & & $\begin{array}{l}\text { II } \\
\text { II } \\
\text { II } \\
\text { II } \\
\text { II }\end{array}$ & $\begin{array}{lll}7 & 49 & 23 . \\
8 & 17 & 174 \\
8 & 434 \\
8 & 46 & 22.734 \\
9 & 7 & 39.051 \\
9 & 46 & 32.361\end{array}$ & $\begin{array}{l}+.134 \\
+.154 \\
+.074 \\
+.191 \\
+.141\end{array}$ & $\begin{array}{l}\mathbf{S} \\
\mathbf{S} \\
\mathbf{S} \\
\mathbf{S} \\
\mathbf{S}\end{array}$ & $\begin{array}{rrr}+18 & 1 & 32.34 \\
+17 & 19 & 57.92 \\
+16 & 15 & 38.62 \\
+15 & 15 & 30.27 \\
+12 & 58 & 23.23\end{array}$ & $\begin{array}{l}-.26 \\
+.12 \\
\pm .18 \\
-.53 \\
-.37\end{array}$ \\
\hline $\begin{array}{l}2.9 \\
8.9\end{array}$ & $\begin{array}{l}\mathrm{Ry} \\
\mathrm{Bn}\end{array}$ & $\stackrel{\mathrm{E}}{\mathrm{W}}$ & II & $\begin{array}{rrr}9 & 50 & 53.238 \\
10 & 17 & 3.007\end{array}$ & $\begin{array}{l}+.048 \\
+.197\end{array}$ & $\stackrel{\mathrm{S}}{\mathrm{S}}$ & $\begin{array}{r}+124058.19 \\
+1048 \quad 0.61\end{array}$ & $\begin{array}{l}-.51 \\
-.09\end{array}$ \\
\hline
\end{tabular}




\begin{tabular}{|c|c|c|c|c|c|c|c|c|c|c|}
\hline \multicolumn{11}{|c|}{$\mathbf{M} \mathbf{A} \mathbf{S}$} \\
\hline \multirow[b]{2}{*}{ Date } & \multirow[b]{2}{*}{ Obsr. } & \multirow[b]{2}{*}{ Cl. } & \multirow[b]{2}{*}{ Limb } & \multirow{2}{*}{$\begin{array}{l}\text { App. R. A. } \\
\text { of Center }\end{array}$} & \multicolumn{2}{|c|}{ Corr. to- } & \multirow[b]{2}{*}{ Limb } & \multirow{2}{*}{$\begin{array}{l}\text { App. Decl. } \\
\text { of Center }\end{array}$} & \multicolumn{2}{|c|}{ Corr. to- } \\
\hline & & & & & $\begin{array}{l}\text { Am. } \\
\text { Eph. }\end{array}$ & Ross & & & $\begin{array}{l}\text { Am. } \\
\text { Eph. }\end{array}$ & Ross \\
\hline \begin{tabular}{rr}
\multicolumn{2}{c}{1913} \\
Dec. & 3.6 \\
& 4.6 \\
& 5.6 \\
& 8.6 \\
& 9.6
\end{tabular} & $\begin{array}{l}\mathrm{P} \\
\mathrm{P} \\
\mathrm{M} \\
\mathrm{Ep}\end{array}$ & $\mathrm{E}$ & $\begin{array}{l}\text { I, II } \\
\text { I, II } \\
\text { I, II } \\
\text { I, II }\end{array}$ & $\begin{array}{llc}\mathrm{h} & \mathrm{m} & \mathrm{s} \\
7 & 46 & 21.138 \\
7 & 45 & 54.648 \\
7 & 45 & 24.432 \\
7 & 44 & \\
7 & 42 & 46.936\end{array}$ & $\begin{array}{r}\mathrm{s} \\
+0.158 \\
+\quad .228 \\
+\quad .232 \\
+\quad .156\end{array}$ & $\begin{array}{c}\mathrm{s} \\
-0.032 \\
+.036 \\
+.038 \\
-.045\end{array}$ & $\begin{array}{l}\mathrm{C} \\
\mathrm{C} \\
\mathrm{C} \\
\mathrm{C} \\
\mathrm{C}\end{array}$ & \begin{tabular}{rrc}
\multicolumn{1}{r}{} & \multicolumn{1}{c}{} & \multicolumn{1}{c}{} \\
+23 & 56 & 11.22 \\
+24 & 0 & 12.89 \\
+24 & 4 & 23.70 \\
+24 & 17 & 42.39 \\
+24 & 22 & 24.66
\end{tabular} & $\begin{array}{r}\prime \prime \\
-0.58 \\
-.81 \\
-.50 \\
-1.31 \\
-.34\end{array}$ & $\begin{array}{r}\quad l \\
+0.09 \\
.13 \\
+.19 \\
. .60 \\
+.37\end{array}$ \\
\hline $\begin{array}{l}11.6 \\
13.6 \\
15.6 \\
27.5 \\
29.5\end{array}$ & $\begin{array}{l}\mathrm{Ep} \\
\mathrm{M} \\
\mathrm{Ep} \\
\mathrm{M} \\
\mathrm{P}\end{array}$ & & $\begin{array}{l}\text { I, II } \\
\text { I, II } \\
\text { I, II } \\
\text { I, II } \\
\text { I, II }\end{array}$ & $\begin{array}{rrr}7 & 41 & 6.628 \\
7 & 39 & 12.272 \\
7 & 37 & 3.983 \\
7 & 20 & 6.151 \\
7 & 16 & 45.284\end{array}$ & $\begin{array}{l}+.198 \\
+.302 \\
+.203 \\
+.361 \\
+.224\end{array}$ & $\begin{array}{l}-.006 \\
+.095 \\
+.007 \\
+.0135 \\
-.002\end{array}$ & $\begin{array}{l}\mathrm{C} \\
\mathrm{C} \\
\mathrm{C} \\
\mathrm{C} \\
\mathrm{C}\end{array}$ & $\begin{array}{rrr}+24 & 32 & 6.09 \\
+24 & 42 & 10.81 \\
+24 & 52 & 32.90 \\
+25 & 55 & 53.18 \\
+26 & 5 & 39.70\end{array}$ & $\begin{array}{l}=.91 \\
=.59 \\
=1.00 \\
=.92 \\
-.70\end{array}$ & $\begin{array}{l}-.19 \\
+.14 \\
\overline{.} .27 \\
+.19 \\
+.02\end{array}$ \\
\hline \begin{tabular}{rr}
\multicolumn{2}{c}{1914} \\
Jan. & 13.5 \\
15.5 \\
17.5 \\
21.4
\end{tabular} & $\begin{array}{l}\mathrm{M} \\
\mathrm{Ep} \\
\mathrm{P} \\
\mathrm{P}\end{array}$ & & $\begin{array}{l}\text { I, II } \\
\text { I, II } \\
\text { I, II } \\
\text { I, II }\end{array}$ & $\begin{array}{rrr}6 & 51 & 3.430 \\
6 & 47 & 55.242 \\
6 & 44 & 56.016 \\
6 & 39 & 28.660\end{array}$ & $\begin{array}{l}+.290 \\
+.112 \\
+.096 \\
+.110\end{array}$ & $\begin{array}{l}+.067 \\
-.107 \\
-.122 \\
-.098\end{array}$ & $\begin{array}{l}\mathrm{C} \\
\mathrm{C} \\
\mathrm{C} \\
\mathrm{C}\end{array}$ & $\begin{array}{rrr}+26 & 58 & 37.31 \\
+27 & 2 & 26.99 \\
+27 & 5 & 33.03 \\
+27 & 9 & 34.35\end{array}$ & $\begin{array}{l} \pm .11 \\
\pm .61 \\
\pm .33 \\
-.75\end{array}$ & $\begin{array}{l}+.70 \\
\pm .04 \\
\pm .89 \\
-.22\end{array}$ \\
\hline $\begin{array}{r}26.4 \\
27.4 \\
28.4 \\
\\
29.4 \\
\text { Feb. } \quad 7.4\end{array}$ & $\begin{array}{l}\mathrm{Ep} \\
\mathrm{P} \\
\mathrm{M} \\
\mathrm{Ep} \\
\mathrm{M}\end{array}$ & $\stackrel{\mathrm{E}}{\mathrm{W}}$ & $\begin{array}{l}\text { I, II } \\
\text { I, II } \\
\text { I, II } \\
\text { I, II } \\
\text { I, II }\end{array}$ & $\begin{array}{llr}6 & 33 & 47.701 \\
6 & 32 & 49.644 \\
6 & 31 & 55.116 \\
6 & 31 & 3.910 \\
6 & 26 & 6.115\end{array}$ & $\begin{array}{l}+.001 \\
+.084 \\
+.236 \\
+.100 \\
+.135\end{array}$ & $\begin{array}{l}-.197 \\
-.112 \\
+.042 \\
-.091 \\
-.034\end{array}$ & $\begin{array}{l}C \\
C \\
C \\
C \\
C\end{array}$ & $\begin{array}{rrr}+27 & 11 & 4.15 \\
+27 & 10 & 56.39 \\
+27 & 10 & 42.32 \\
+27 & 10 & 20.98 \\
+27 & 2 & 53.08\end{array}$ & $\begin{array}{l} \pm .05 \\
\pm .71 \\
\pm .38 \\
+.22 \\
+.58\end{array}$ & $\begin{array}{l}+.54 \\
+.23 \\
+.09 \\
+.25 \\
+.99\end{array}$ \\
\hline 1916 9.4 & $\stackrel{\mathrm{M}}{\mathrm{P}}$ & W & $\begin{array}{l}\text { 1, II } \\
\text { I, II }\end{array}$ & 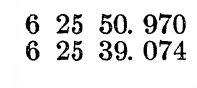 & $\begin{array}{l}+.230 \\
+.094\end{array}$ & $\begin{array}{l}+.064 \\
-.070\end{array}$ & $\begin{array}{l}\mathrm{C} \\
\mathrm{C}\end{array}$ & $\begin{array}{lll}+27 & 1 & 39.21 \\
+27 & 0 & 23.24\end{array}$ & $\begin{array}{l}-.59 \\
-.26\end{array}$ & $\begin{array}{l}\overline{+} .19 \\
+.14\end{array}$ \\
\hline $\begin{array}{r}\text { Jan. } 8.6 \\
18.6\end{array}$ & $\begin{array}{l}\mathrm{Sr} \\
\mathrm{M}\end{array}$ & $\mathrm{E}$ & $\begin{array}{l}\text { I, II } \\
\text { I, II }\end{array}$ & $\begin{array}{rrr}10 & 11 & 26.666 \\
10 & 5 & 2.630\end{array}$ & $\begin{array}{l}+.116 \\
+.160\end{array}$ & $\begin{array}{l}+.076 \\
+.120\end{array}$ & $\begin{array}{l}\mathrm{C} \\
\mathrm{C}\end{array}$ & $\begin{array}{rrr}+1517 & 8.98 \\
+16 & 14 & 26.72\end{array}$ & $\begin{array}{l}-1.02 \\
-.58\end{array}$ & $\begin{array}{r}-.47 \\
.00\end{array}$ \\
\hline $\begin{array}{r}20.6 \\
23.6 \\
\text { Feb. } \quad 7.5 \\
9.5 \\
14.5\end{array}$ & $\begin{array}{l}\mathrm{Sr} \\
\mathrm{Sr} \\
\mathrm{P} \\
\mathrm{Sr} \\
\mathrm{P}\end{array}$ & $\underset{W}{\mathrm{E}}$ & $\begin{array}{l}\text { I, II } \\
\text { I, II } \\
\text { I, II } \\
\text { I, II } \\
\text { I, II }\end{array}$ & $\begin{array}{rrr}10 & 3 & 8.903 \\
9 & 59 & 56.788 \\
9 & 38 & 52.640 \\
9 & 35 & 42.022 \\
9 & 27 & 45.426\end{array}$ & $\begin{array}{l}+.133 \\
+.158 \\
+.020 \\
+.032 \\
+.004\end{array}$ & $\begin{array}{l}+.093 \\
+.118 \\
-.020 \\
-.008 \\
-.042\end{array}$ & $\begin{array}{l}\mathrm{C} \\
\mathbf{C} \\
\mathrm{C} \\
\mathbf{C} \\
\mathrm{C}\end{array}$ & 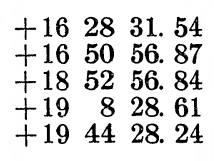 & $\begin{array}{l}-.96 \\
-.53 \\
+.54 \\
+.69 \\
+.14\end{array}$ & $\begin{array}{l}\bar{T} .38 \\
+.05 \\
+1.12 \\
+.12 \\
+.69\end{array}$ \\
\hline $\begin{array}{r}15.5 \\
29.4 \\
\text { Mar. } \\
7.4 \\
9.4 \\
10.4\end{array}$ & $\begin{array}{l}\mathrm{Sr} \\
\mathrm{P} \\
\mathrm{Sr} \\
\mathrm{S} \\
\mathrm{Sr}\end{array}$ & & $\begin{array}{l}\text { I, II } \\
\text { I, II } \\
\text { I, II } \\
\text { I, II } \\
\text { I, II }\end{array}$ & $\begin{array}{rrr}9 & 26 & 11.613 \\
9 & 7 & 2.054 \\
9 & 0 & 29.061 \\
8 & 59 & 4.675 \\
8 & 58 & 27.343\end{array}$ & $\begin{array}{l}+.033 \\
+.004 \\
+.019 \\
+.035 \\
+.013\end{array}$ & $\begin{array}{l}-.005 \\
-.028 \\
-.048 \\
-.063 \\
-.014\end{array}$ & $\begin{array}{l}\mathbf{C} \\
\mathbf{C} \\
\mathbf{C} \\
\mathbf{C} \\
\mathbf{C}\end{array}$ & $\begin{array}{rrr}+19 & 51 & 2.95 \\
+20 & 56 & 53.30 \\
+21 & 9 & 39.28 \\
+21 & 10 & 53.18 \\
+21 & 11 & 7.21\end{array}$ & $\begin{array}{l}-1.05 \\
\pm .10 \\
+.08 \\
-.33 \\
-.49\end{array}$ & $\begin{array}{l}+.50 \\
+.36 \\
+.50 \\
+.07 \\
+.10\end{array}$ \\
\hline $\begin{array}{l}11.4 \\
13.4 \\
16.4 \\
17.4 \\
23.4\end{array}$ & $\begin{array}{l}\mathrm{P} \\
\mathrm{M} \\
\mathrm{P} \\
\mathrm{Sr} \\
\mathrm{M}\end{array}$ & & $\begin{array}{l}\text { I, II } \\
\text { I, II } \\
\text { I, II } \\
\text { I, II }\end{array}$ & $\begin{array}{lll}8 & 57 & 53.170 \\
8 & 57 & \\
8 & 55 & 50.438 \\
8 & 55 & 35.415 \\
8 & 55 & 9.301\end{array}$ & $\begin{array}{r}.000 \\
-.002 \\
+.025 \\
+.021\end{array}$ & $\begin{array}{l}-.026 \\
-.024 \\
+.004 \\
+.005\end{array}$ & $\begin{array}{l}\mathrm{C} \\
\mathbf{C} \\
\mathbf{C} \\
\mathbf{C} \\
\mathrm{C}\end{array}$ & $\begin{array}{rrr}+21 & 11 & 7.07 \\
+21 & 10 & 21.75 \\
+21 & 7 & 30.91 \\
+21 & 6 & 6.97 \\
+20 & 53 & 26.73\end{array}$ & $\begin{array}{l} \pm .07 \\
\pm .65 \\
=.09 \\
=.33 \\
-.17\end{array}$ & $\begin{array}{l}+.46 \\
+.28 \\
+.27 \\
+.02 \\
+.14\end{array}$ \\
\hline \begin{tabular}{rr}
\multicolumn{1}{c}{1918} \\
Feb. 9.6 \\
11.6 \\
12.6 \\
13.6
\end{tabular} & $\begin{array}{l}\mathrm{P} \\
\mathrm{M} \\
\mathrm{P} \\
\mathrm{Sr}\end{array}$ & & $\begin{array}{l}\text { I, II } \\
\text { I, II } \\
\text { I, II } \\
\text { I, II }\end{array}$ & $\begin{array}{llr}12 & 16 & 4.174 \\
12 & 15 & 26.352 \\
12 & 15 & 3.054 \\
12 & 14 & 36.793\end{array}$ & $\begin{array}{l}+.044 \\
+.032 \\
+.044 \\
+.013\end{array}$ & $\begin{array}{l}+.120 \\
+.111 \\
+.124 \\
+.095\end{array}$ & $\begin{array}{l}\mathrm{C} \\
\mathrm{C} \\
\mathrm{C} \\
\mathrm{C}\end{array}$ & 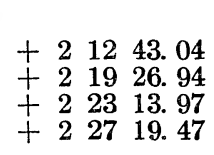 & $\begin{array}{l}-.56 \\
=.96 \\
=1.43 \\
-.13\end{array}$ & $\begin{array}{l}-.78 \\
=1.20 \\
=1.68 \\
-.38\end{array}$ \\
\hline 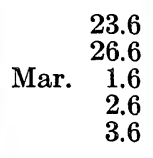 & $\begin{array}{l}\mathrm{P} \\
\mathrm{Sr} \\
\mathrm{Sr} \\
\mathrm{P} \\
\mathrm{M}\end{array}$ & & $\begin{array}{l}\text { I, II } \\
\text { I, II } \\
\text { I, II } \\
\text { I, II }\end{array}$ & $\begin{array}{rrr}12 & 7 & 36.528 \\
12 & 5 & \\
12 & 1 & 16.157 \\
12 & 0 & 4.588 \\
11 & 58 & 51.040\end{array}$ & $\begin{array}{l}+.008 \\
-.063 \\
-.102 \\
-.030\end{array}$ & $\begin{array}{l}+.101 \\
+.035 \\
+.003 \\
+.070\end{array}$ & $\begin{array}{l}\mathrm{C} \\
\mathrm{C} \\
\mathrm{C} \\
\mathrm{C} \\
\mathrm{C}\end{array}$ & 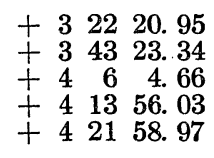 & $\begin{array}{l}\bar{T} .85 \\
+.14 \\
+.46 \\
+.67 \\
+1.67\end{array}$ & $\begin{array}{l}-1.19 \\
. .23 \\
+.07 \\
+1.06 \\
+1.27\end{array}$ \\
\hline $\begin{array}{r}5.5 \\
7.5 \\
15.5 \\
23.5 \\
25.5\end{array}$ & $\begin{array}{l}\mathrm{Sr} \\
\mathrm{M} \\
\mathrm{Sr} \\
\mathrm{Sr} \\
\mathrm{Sr}\end{array}$ & $\mathrm{W}$ & $\begin{array}{l}\text { I, II } \\
\text { I, II } \\
\text { I, II } \\
\text { I, II } \\
\text { I, II }\end{array}$ & $\begin{array}{lll}11 & 56 & 17.939 \\
11 & 53 & 37.888 \\
11 & 42 & 10.689 \\
11 & 30 & 31.763 \\
11 & 27 & 44.949\end{array}$ & $\begin{array}{l}-.061 \\
-.072 \\
-.121 \\
-.087 \\
-.121\end{array}$ & $\begin{array}{l}+.040 \\
+.030 \\
+.015 \\
+.018 \\
+.016\end{array}$ & $\begin{array}{l}\mathrm{C} \\
\mathrm{C} \\
\mathrm{C} \\
\mathrm{C} \\
\mathrm{C}\end{array}$ & $\begin{array}{rrr}+ & 38 & 19.42 \\
+ & 455 & 3.01 \\
+ & 62 & 45.28 \\
+7 & 452.23 \\
+ & 78 & 34.23\end{array}$ & $\begin{array}{l}+.12 \\
+.31 \\
+.58 \\
+.73 \\
+.13\end{array}$ & $\begin{array}{l}-.29 \\
\bar{t} .11 \\
+.11 \\
\pm .23 \\
-.38\end{array}$ \\
\hline
\end{tabular}




\begin{tabular}{|c|c|c|c|c|c|c|c|c|c|c|}
\hline \multicolumn{11}{|c|}{$\mathbf{M} \mathbf{A} \mathbf{R}$} \\
\hline \multirow[b]{2}{*}{ Date } & \multirow[b]{2}{*}{ Obsr. } & \multirow[b]{2}{*}{$\mathrm{Cl}}$. & \multirow[b]{2}{*}{ Limb } & \multirow{2}{*}{$\underset{\text { of Center }}{\text { App. R. A. }}$} & \multicolumn{2}{|c|}{ Corr. to- } & \multirow[b]{2}{*}{$\operatorname{Limb}$} & \multirow{2}{*}{$\begin{array}{l}\text { App. Decl. } \\
\text { of Centẹr }\end{array}$} & \multicolumn{2}{|c|}{ Corr. to- } \\
\hline & & & & & $\begin{array}{l}\text { Am. } \\
\text { Eph. }\end{array}$ & Ross & & & $\begin{array}{l}\text { Am. } \\
\text { Eph. }\end{array}$ & Ross \\
\hline $\begin{aligned} & 1918 \\
& \text { Mar. } 26.5 \\
& 27.5 \\
& 28.5 \\
& \text { Apr. } 18.4 \\
& 21.4\end{aligned}$ & $\begin{array}{l}\mathrm{P} \\
\mathrm{P} \\
\mathrm{M} \\
\mathrm{M} \\
\mathrm{M}\end{array}$ & W & $\begin{array}{l}\text { I, II } \\
\text { I, II } \\
\text { I, II } \\
\text { I, II } \\
\text { I, II }\end{array}$ & \begin{tabular}{lrr}
$\mathrm{h}$ & $\mathrm{m}$ & \multicolumn{1}{c}{$\mathrm{s}$} \\
11 & 26 & 23.589 \\
11 & 25 & 3.718 \\
11 & 23 & 45.448 \\
11 & 5 & 28. \\
11 & 4 & 32.487 \\
& &
\end{tabular} & $\begin{array}{l}s \\
-0.101 \\
-.122 \\
-.172 \\
-.066 \\
-.053\end{array}$ & $\begin{array}{r}\mathrm{s} \\
+0.003 \\
-\quad .018 \\
-.068 \\
+.028 \\
+.039\end{array}$ & $\begin{array}{l}\mathbf{C} \\
\mathbf{C} \\
\mathbf{C} \\
\mathbf{C} \\
\mathbf{C}\end{array}$ & $\begin{array}{rcc} & \circ & \prime \\
+7 & 25 & 5.33 \\
+ & 31 & 21.72 \\
+ & 37 & 23.06 \\
+ & 37 \\
+ & 35 & 36.30 \\
+ & 832 & 51.22\end{array}$ & $\begin{array}{r} \\
\quad \prime \prime \\
+0.63 \\
+.82 \\
+1.16 \\
+.50 \\
+\quad .32\end{array}$ & $\begin{array}{r} \\
+1 \prime \\
+0.12 \\
+.30 \\
+.64 \\
-.02 \\
-.20\end{array}$ \\
\hline${ }_{1920}^{27.4}$ & $\mathrm{Sr}$ & & I, II & 11357.953 & -.097 & -.006 & C & +81944.81 & +.71 & +.18 \\
\hline $\begin{array}{r}9.6 \\
\text { Mar. } \\
22.6 \\
23.6\end{array}$ & $\begin{array}{l}\mathrm{M} \\
\mathrm{M} \\
\mathrm{Bn}\end{array}$ & & $\begin{array}{l}\text { I, II } \\
\text { I, II } \\
\text { I, II }\end{array}$ & $\begin{array}{rrr}14 & 29 & 17.285 \\
14 & 28 & 5.328 \\
14 & 27 & 38.804\end{array}$ & $\begin{array}{l}-.105 \\
=.152 \\
-.236\end{array}$ & $\begin{array}{l}+.062 \\
+.045 \\
+.036\end{array}$ & $\begin{array}{l}\mathrm{C} \\
\mathrm{C} \\
\mathrm{C}\end{array}$ & $\begin{array}{lll}-12 & 18 & 50.33 \\
-12 & 17 & 23.86 \\
-12 & 15 & 51.54\end{array}$ & $\begin{array}{l}+.37 \\
+\quad .94 \\
+1.66\end{array}$ & $\begin{array}{l}-.39 \\
-.05 \\
+.65\end{array}$ \\
\hline $\begin{array}{l}24.6 \\
26.6 \\
27.6 \\
30.6 \\
31.6\end{array}$ & $\begin{array}{l}\mathrm{P} \\
\mathrm{Ry} \\
\mathrm{P} \\
\mathrm{Bn} \\
\mathrm{Ry}\end{array}$ & $\begin{array}{l}\mathrm{W} \\
\mathrm{E}\end{array}$ & $\begin{array}{l}\text { I, II } \\
\text { I, II } \\
\text { I, II } \\
\text { I, II }\end{array}$ & $\begin{array}{rrr}14 & 27 & 9.500 \\
14 & 26 & 1.478 \\
14 & 25 & 23.102 \\
14 & 23 & 10.048 \\
14 & 22 & \end{array}$ & $\begin{array}{l}-.080 \\
=.142 \\
=.068 \\
-.192\end{array}$ & $\begin{array}{l}+.122 \\
+.065 \\
+.141 \\
+.026\end{array}$ & $\begin{array}{l}\mathrm{C} \\
\mathrm{C} \\
\mathrm{C} \\
\mathrm{C} \\
\mathrm{C}\end{array}$ & $\begin{array}{rrr}-12 & 14 & 8.87 \\
-12 & 10 & 5.64 \\
-12 & 7 & 45.67 \\
-11 & 59 & 36.01 \\
-11 & 56 & 28.75\end{array}$ & $\begin{array}{l}+.63 \\
+.26 \\
+.53 \\
+.19 \\
+.95\end{array}$ & $\begin{array}{l}=.39 \\
=.80 \\
=.55 \\
=.94 \\
-.20\end{array}$ \\
\hline 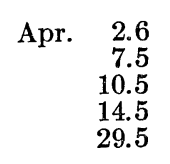 & $\begin{array}{l}\mathrm{Bn} \\
\mathrm{P} \\
\mathrm{P} \\
\mathrm{Ry} \\
\mathrm{Bn}\end{array}$ & & $\begin{array}{l}\text { I, II } \\
\text { I, II } \\
\text { I, II } \\
\text { I, II } \\
\text { I, II }\end{array}$ & 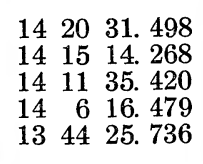 & $\begin{array}{l}-.182 \\
=.122 \\
=.170 \\
=.191 \\
-.334\end{array}$ & $\begin{array}{l}+.043 \\
+.113 \\
+.070 \\
+.055 \\
+.084\end{array}$ & $\begin{array}{l}\text { C } \\
\text { C } \\
\text { C } \\
\text { C } \\
\text { C }\end{array}$ & $\begin{array}{lll}-11 & 49 & 42.20 \\
-11 & 29 & 38.34 \\
-11 & 15 & 40.70 \\
-10 & 55 & 12.68 \\
-9 & 31 & 34.97\end{array}$ & $\begin{array}{l}+.80 \\
+.86 \\
+.70 \\
+.92 \\
+1.63\end{array}$ & $\begin{array}{l}=.39 \\
=.42 \\
=.63 \\
+.48 \\
+.05\end{array}$ \\
\hline $\begin{array}{r}\text { May } 1.5 \\
3.5 \\
4.4 \\
5.4 \\
14.4\end{array}$ & $\begin{array}{l}\mathrm{P} \\
\mathrm{M} \\
\mathrm{P} \\
\mathrm{Ry} \\
\mathrm{Ry}\end{array}$ & & $\begin{array}{l}\text { I, II } \\
\text { I, II } \\
\text { I, II } \\
\text { I, II } \\
\text { I, II }\end{array}$ & 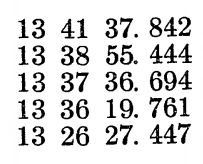 & $\begin{array}{l}=.228 \\
=.236 \\
=.246 \\
=.249 \\
-.213\end{array}$ & $\begin{array}{l}+.018 \\
+.007 \\
+.004 \\
+.008 \\
+.015\end{array}$ & $\begin{array}{l}\mathrm{C} \\
\mathrm{C} \\
\mathrm{C} \\
\mathrm{C} \\
\mathrm{C}\end{array}$ & 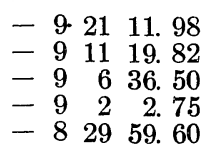 & $\begin{array}{l}+1.32 \\
+\quad .88 \\
+1.10 \\
+1.55 \\
+3.00\end{array}$ & $\begin{array}{l}-.27 \\
-.71 \\
-.49 \\
-.05 \\
+1.41\end{array}$ \\
\hline $\begin{array}{r}15.4 \\
17.4 \\
22.4 \\
1922\end{array}$ & $\begin{array}{l}\mathrm{Bn} \\
\mathrm{Bn} \\
\mathrm{P}\end{array}$ & & $\begin{array}{l}\text { I, II } \\
\text { I, II } \\
\text { I, II }\end{array}$ & 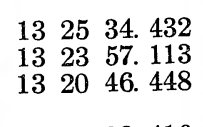 & $\begin{array}{l}-.258 \\
-.267 \\
-.242\end{array}$ & $\begin{array}{l}-.032 \\
=.046 \\
-.030\end{array}$ & $\begin{array}{l}\mathrm{C} \\
\mathrm{C} \\
\mathrm{C}\end{array}$ & $\begin{array}{rrr}-8 & 27 & 36.21 \\
-8 & 23 & 33.68 \\
-818 & 8.93\end{array}$ & $\begin{array}{l}+1.89 \\
+1.62 \\
+1.17\end{array}$ & $\begin{array}{l}+.31 \\
+.06 \\
-.37\end{array}$ \\
\hline $\begin{array}{l}1922 \\
\text { May } 1.6\end{array}$ & $\mathrm{Bn}$ & & I, II & 173822.416 & +.086 & & $\mathrm{C}$ & $\begin{array}{lll}-24 & 2 & 35.79\end{array}$ & -.39 & \\
\hline $\begin{array}{l}10.6 \\
15.6 \\
19.6 \\
22.6 \\
24.6\end{array}$ & $\begin{array}{l}\text { Ry } \\
\text { Bn } \\
\text { Ry } \\
\text { Ry } \\
\text { Ry }\end{array}$ & & $\begin{array}{l}\text { I, II } \\
\text { I, II } \\
\text { I, II } \\
\text { I, II } \\
\text { I, II }\end{array}$ & 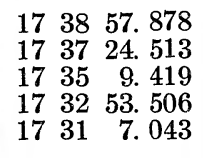 & $\begin{array}{l}+.038 \\
+.163 \\
+.099 \\
+.076 \\
+.023\end{array}$ & & $\begin{array}{l}\mathrm{C} \\
\mathrm{C} \\
\mathrm{C} \\
\mathrm{C} \\
\mathrm{C}\end{array}$ & $\begin{array}{rrr}-24 & 30 & 10.00 \\
-24 & 46 & 10.67 \\
-24 & 59 & 4.36 \\
-25 & 8 & 38.04 \\
-25 & 14 & 52.31\end{array}$ & $\begin{array}{l}-.20 \\
\bar{T} .77 \\
+.04 \\
+.06 \\
+.19\end{array}$ & \\
\hline $\begin{array}{r}27.5 \\
28.5 \\
29.5 \\
30.5 \\
\text { June } \quad 7.5\end{array}$ & $\begin{array}{l}\mathrm{P} \\
\mathrm{M} \\
\mathrm{Bn} \\
\mathrm{Ry} \\
\mathrm{Ry}\end{array}$ & & $\begin{array}{l}\text { I, II } \\
\text { I, II } \\
\text { I, II } \\
\text { I, II } \\
\text { I, II }\end{array}$ & $\begin{array}{rrr}17 & 28 & 5.146 \\
17 & 26 & 59.024 \\
17 & 25 & 50.340 \\
17 & 24 & 39.157 \\
17 & 14 & 0.427\end{array}$ & $\begin{array}{l}+.026 \\
+.054 \\
+.090 \\
+.057 \\
+.017\end{array}$ & & $\begin{array}{l}\mathrm{C} \\
\mathrm{C} \\
\mathrm{C} \\
\mathrm{C} \\
\mathrm{C}\end{array}$ & $\begin{array}{llll}-25 & 23 & 55.12 \\
-25 & 26 & 48.07 \\
-25 & 29 & 38.15 \\
-25 & 32 & 23 . & 56 \\
-25 & 51 & 15.22\end{array}$ & $\begin{array}{l}\bar{T} .82 \\
+.03 \\
-.25 \\
\bar{t} .16 \\
+.08\end{array}$ & \\
\hline $\begin{array}{l}10.5 \\
12.5 \\
15.5 \\
22.5 \\
23.4\end{array}$ & $\begin{array}{l}\mathrm{P} \\
\mathrm{P} \\
\mathrm{Ry} \\
\mathrm{P} \\
\mathrm{P}\end{array}$ & $\begin{array}{l}\mathrm{E} \\
\mathrm{W}\end{array}$ & $\begin{array}{l}\text { I, II } \\
\text { I, II } \\
\text { I, II } \\
\text { I, II } \\
\text { I, II }\end{array}$ & $\begin{array}{lrr}17 & 9 & 40.110 \\
17 & 6 & 44.172 \\
17 & 2 & 20.615 \\
16 & 52 & 35 . \\
16 & 51 & 18.261 \\
16 & 53 & 18 .\end{array}$ & $\begin{array}{l}+.010 \\
+.042 \\
+.045 \\
+.004 \\
-.079\end{array}$ & . & $\begin{array}{l}\mathrm{C} \\
\mathrm{C} \\
\mathrm{C} \\
\mathrm{C} \\
\mathrm{C}\end{array}$ & $\begin{array}{rrr}-25 & 56 & 34.83 \\
-25 & 59 & 32.99 \\
-26 & 3 & 7.48 \\
-26 & 7 & 34.17 \\
-26 & 7 & 49.90\end{array}$ & $\begin{array}{l}+.47 \\
+.41 \\
-.18 \\
-.67 \\
-1.50\end{array}$ & \\
\hline $\begin{array}{rr} & 25.4 \\
& 29.4 \\
& 30.4 \\
\text { July } & 5.4\end{array}$ & $\begin{array}{l}\mathrm{M} \\
\mathrm{Ry} \\
\mathrm{Bn} \\
\mathrm{Ry}\end{array}$ & & $\begin{array}{l}\text { I, II } \\
\text { I, II } \\
\text { I, II } \\
\text { I, II }\end{array}$ & $\begin{array}{lll}16 & 48 & 50.646 \\
16 & 44 & 26.470 \\
16 & 43 & 27.820 \\
16 & 39 & 23.383\end{array}$ & $\begin{array}{l}+.036 \\
+.040 \\
+.110 \\
+.083\end{array}$ & & $\begin{array}{l}\mathrm{C} \\
\mathrm{C} \\
\mathrm{C} \\
\mathrm{C}\end{array}$ & $\begin{array}{rrr}-26 & 8 & 5.42 \\
-26 & 7 & 55.08 \\
-26 & 7 & 46.08 \\
-26 & 6 & 49.93\end{array}$ & $\begin{array}{l}-.92 \\
=.78 \\
=.48 \\
-.53\end{array}$ & \\
\hline \begin{tabular}{cc}
\multicolumn{2}{c}{1924} \\
July & 10.6 \\
& 14.6 \\
& 17.6 \\
& 18.6 \\
& 19.6
\end{tabular} & $\begin{array}{l}\mathrm{P} \\
\mathrm{Bn} \\
\mathrm{P} \\
\mathrm{Ry} \\
\mathrm{Bn}\end{array}$ & W & $\begin{array}{l}\text { I, II } \\
\text { I, II } \\
\text { I, II } \\
\text { I, II } \\
\text { I, II }\end{array}$ & $\begin{array}{rrr}22 & 32 & 3.731 \\
22 & 34 & 36.346 \\
22 & 36 & 1.468 \\
22 & 36 & 24.086 \\
22 & 36 & 43.638\end{array}$ & $\begin{array}{l}-.079 \\
+.006 \\
+.002 \\
+.046 \\
-.002\end{array}$ & & $\begin{array}{l}\mathrm{C} \\
\mathrm{C} \\
\mathrm{C} \\
\mathrm{C} \\
\mathrm{C}\end{array}$ & $\begin{array}{rrr}-14 & 44 & 52.04 \\
-14 & 46 & 32.08 \\
-14 & 50 & 21.69 \\
-14 & 52 & 9.49 \\
-14 & 54 & 11.84\end{array}$ & $\begin{array}{l}-.04 \\
\overline{-} .78 \\
+.51 \\
-.09 \\
-.14\end{array}$ & \\
\hline
\end{tabular}




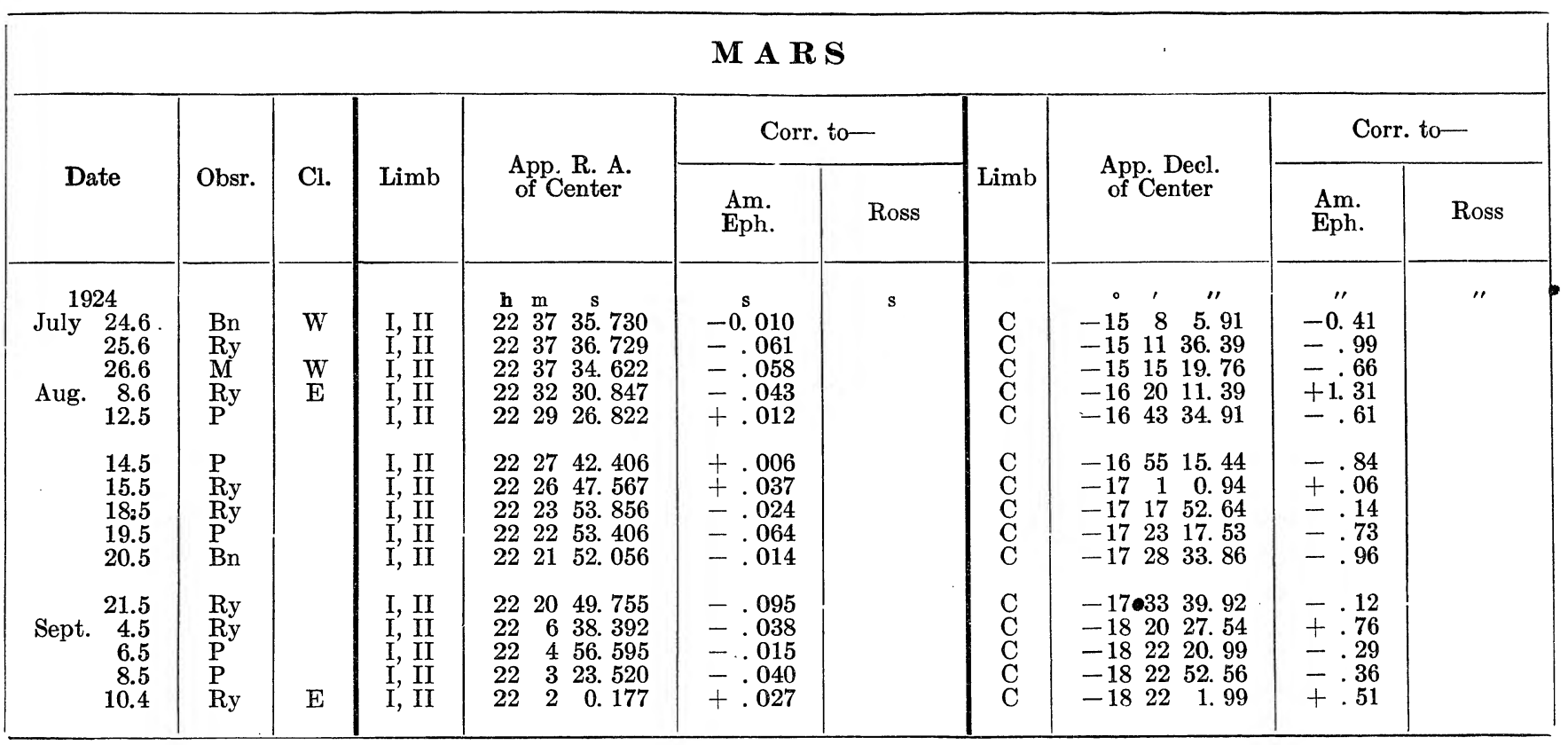

$137506^{\circ}-33-10$ 


\begin{tabular}{|c|c|c|c|c|c|c|c|c|}
\hline \multicolumn{9}{|c|}{ J U P I T E R } \\
\hline Date & Obsr. & $\mathrm{Cl}$. & Limb & $\begin{array}{l}\text { App. R. A. } \\
\text { of Center }\end{array}$ & $\begin{array}{l}\text { Corr. to } \\
\text { Am. Eph. }\end{array}$ & Limb & $\begin{array}{l}\text { App. Decl. } \\
\text { of Center }\end{array}$ & $\begin{array}{l}\text { Corr. to } \\
\text { Am. Eph. }\end{array}$ \\
\hline \begin{tabular}{ll}
\multicolumn{1}{c}{1914} \\
July & 16.6 \\
& 18.6 \\
& 19.6 \\
& 20.6 \\
& 21.6
\end{tabular} & $\begin{array}{l}\mathrm{P} \\
\mathrm{P} \\
\mathrm{S} \\
\mathrm{M} \\
\mathrm{P}\end{array}$ & $\mathbf{E}$ & $\begin{array}{l}\text { I, II } \\
\text { 1, II } \\
\text { I, II } \\
\text { I, II } \\
\text { I, II }\end{array}$ & $\begin{array}{ccc}\mathrm{h} & \mathrm{m} & \mathrm{s} \\
21 & 32 & 10.270 \\
21 & 31 & 21.324 \\
21 & 30 & 56.090 \\
21 & 30 & 30.387 \\
21 & 30 & 4.304\end{array}$ & $\begin{array}{r}\mathrm{s} \\
+0.010 \\
+.034 \\
+.010 \\
+.013 \\
+.024\end{array}$ & $\begin{array}{l}\mathrm{C} \\
\mathrm{C} \\
\mathrm{C} \\
\mathrm{C} \\
\mathrm{C}\end{array}$ & \begin{tabular}{rrr}
$\circ$ & \multicolumn{1}{c}{${ }^{\prime \prime}$} \\
-15 & 36 & 13.59 \\
-15 & 40 & 36.54 \\
-15 & 42 & 49.80 \\
-15 & 45 & 7.29 \\
-15 & 47 & 25.94
\end{tabular} & $\begin{array}{l}\text { + } \\
+0.21 \\
+\quad .76 \\
+2.20 \\
+1.21 \\
+.86\end{array}$ \\
\hline $\begin{array}{rr} & 22.6 \\
\text { Aug. } & 17.5 \\
18.5 \\
19.5 \\
20.5\end{array}$ & $\begin{array}{l}\mathrm{P} \\
\mathrm{Sr} \\
\mathrm{M} \\
\mathrm{S} \\
\mathrm{Sr}\end{array}$ & & $\begin{array}{l}\text { I, II } \\
\text { I', II } \\
\text { I, II } \\
\text { I, II } \\
\text { I, II }\end{array}$ & $\begin{array}{lll}21 & 29 & 37.806 \\
21 & 16 & 42.715 \\
21 & 16 & 12.362 \\
21 & 15 & 42.087 \\
21 & 15 & 12.058\end{array}$ & $\begin{array}{l}+.086 \\
+.025 \\
+.062 \\
+.017 \\
+.018\end{array}$ & $\begin{array}{l}\mathrm{C} \\
\mathrm{C} \\
\mathrm{C} \\
\mathrm{C} \\
\mathrm{C}\end{array}$ & $\begin{array}{rrr}-15 & 49 & 45.79 \\
-16 & 53 & 53.86 \\
-16 & 56 & 16.01 \\
-16 & 58 & 37.51 \\
-17 & 0 & 57.98\end{array}$ & $\begin{array}{l}+.81 \\
+.14 \\
+.89 \\
+.89 \\
+.42\end{array}$ \\
\hline $\begin{array}{rr} & 22.5 \\
& 30.4 \\
& 31.4 \\
\text { Sept. } & 1.4 \\
& 2.4\end{array}$ & $\begin{array}{l}\mathrm{S} \\
\mathrm{S} \\
\mathrm{Sr} \\
\mathrm{M} \\
\mathrm{S}\end{array}$ & $\mathrm{E}$ & $\begin{array}{l}\text { I, II } \\
\text { I, II } \\
\text { I, II } \\
\text { I, II } \\
\text { I, II }\end{array}$ & $\begin{array}{rrr}21 & 14 & 12.637 \\
21 & 10 & 27.186 \\
21 & 10 & 0.753 \\
21 & 9 & 34.686 \\
21 & 9 & 9.140\end{array}$ & $\begin{array}{l}-.023 \\
-.004 \\
+.033 \\
+.004 \\
+.020\end{array}$ & $\begin{array}{l}\mathrm{C} \\
\mathrm{C} \\
\mathrm{C} \\
\mathrm{C} \\
\mathrm{C}\end{array}$ & $\begin{array}{lrr}-17 & 5 & 32.86 \\
-17 & 22 & 35.87 \\
-17 & 24 & 34.13 \\
-17 & 26 & 29.06 \\
-17 & 28 & 22.44\end{array}$ & $\begin{array}{l}+.64 \\
+.43 \\
+.03 \\
+.54 \\
+.16\end{array}$ \\
\hline \begin{tabular}{rr}
\multicolumn{2}{c}{1915} \\
Sept. & 7.5 \\
8.5 \\
10.5 \\
14.5
\end{tabular} & $\begin{array}{l}\mathrm{M} \\
\mathrm{S} \\
\mathrm{S} \\
\mathrm{Sr}\end{array}$ & $\mathrm{W}$ & $\begin{array}{l}\text { I, II } \\
\text { I, II } \\
\text { I, II } \\
\text { I, II }\end{array}$ & $\begin{array}{rrr}23 & 43 & 7.450 \\
23 & 42 & 39.008 \\
23 & 41 & 41.786 \\
23 & 39 & 45.607\end{array}$ & $\begin{array}{l}+.060 \\
+.022 \\
+.046 \\
+.017\end{array}$ & $\begin{array}{l}\mathrm{C} \\
\mathrm{C} \\
\mathrm{C} \\
\mathrm{C}\end{array}$ & 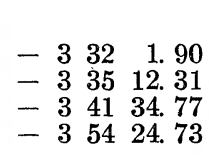 & $\begin{array}{l}+.20 \\
+.09 \\
+.43 \\
-.13\end{array}$ \\
\hline $\begin{array}{l}15.5 \\
16.5 \\
21.5 \\
22.5 \\
23.5\end{array}$ & $\begin{array}{l}\mathrm{P} \\
\mathrm{S} \\
\mathrm{Sr} \\
\mathrm{S} \\
\mathrm{M}\end{array}$ & & $\begin{array}{l}\text { I, II } \\
\text { I, II } \\
\text { I, II } \\
\text { I, II } \\
\text { I, II }\end{array}$ & $\begin{array}{lll}23 & 39 & 16.324 \\
23 & 38 & 47.060 \\
23 & 36 & 20.917 \\
23 & 35 & 51.864 \\
23 & 35 & 22.906\end{array}$ & $\begin{array}{l}-.026 \\
-.010 \\
+.037 \\
+.034 \\
+.006\end{array}$ & $\begin{array}{l}\mathrm{C} \\
\mathrm{C} \\
\mathrm{C} \\
\mathrm{C} \\
\mathrm{C}\end{array}$ & 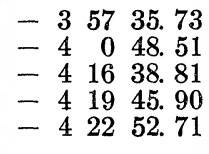 & $\begin{array}{l}+1.17 \\
+.39 \\
+.59 \\
+.70 \\
+.11\end{array}$ \\
\hline $\begin{array}{rr} & 24.5 \\
& 27.5 \\
& 28.5 \\
\text { Oct. } \quad 3.4 \\
. \quad 9.4\end{array}$ & $\begin{array}{l}\mathrm{Sr} \\
\mathrm{M} \\
\mathrm{P} \\
\mathrm{Sr} \\
\mathrm{M}\end{array}$ & & $\begin{array}{l}\text { I, II } \\
\text { I, II } \\
\text { I, II } \\
\text { I, II } \\
\text { I, II }\end{array}$ & $\begin{array}{rrr}23 & 34 & 54.145 \\
23 & 33 & 28.753 \\
23 & 33 & 0.724 \\
23 & 30 & 44.521 \\
23 & 28 & 12.564\end{array}$ & $\begin{array}{l}+.035 \\
+.007 \\
+.004 \\
+.031 \\
+.024\end{array}$ & $\begin{array}{l}\mathrm{C} \\
\mathrm{C} \\
\mathrm{C} \\
\mathrm{C} \\
\mathrm{C}\end{array}$ & $\begin{array}{l}-42557.15 \\
=4351.19 \\
-43758.33 \\
=45212.45 \\
-5 \quad 5 \quad 47.93\end{array}$ & $\begin{array}{l}+.05 \\
+.29 \\
+.17 \\
+.65 \\
+.27\end{array}$ \\
\hline $\begin{array}{r}11.4 \\
1916\end{array}$ & $\mathrm{~s}$ & & I, II & $\begin{array}{lll}23 & 27 & 25.365\end{array}$ & -.005 & C & -51232.79 & +1.11 \\
\hline $\begin{array}{r}1916 \\
\text { Sept. } 23.6 \\
26.6 \\
27.6\end{array}$ & $\begin{array}{l}\mathrm{P} \\
\mathrm{M} \\
\mathrm{P}\end{array}$ & & $\begin{array}{l}\text { I, II } \\
\text { I, II } \\
\text { I, II }\end{array}$ & $\begin{array}{llll}2 & 8 & 54.816 \\
2 & 7 & 48.135 \\
2 & 7 & 24.800\end{array}$ & $\begin{array}{l}+.066 \\
+.015 \\
+.030\end{array}$ & $\begin{array}{l}\mathrm{C} \\
\mathrm{C} \\
\mathrm{C}\end{array}$ & $\begin{array}{r}+112522.62 \\
+11197.00 \\
+11 \quad 1655.90\end{array}$ & $\begin{array}{l}=.08 \\
=.40 \\
-.70\end{array}$ \\
\hline $\begin{array}{rr} & 29.6 \\
\text { Oct. } & 1.5 \\
7.5 \\
12.5 \\
14.5\end{array}$ & $\begin{array}{l}\mathrm{M} \\
\mathrm{Sr} \\
\mathrm{P} \\
\mathrm{M} \\
\mathrm{Sr}\end{array}$ & & $\begin{array}{l}\text { I, II } \\
\text { I, II } \\
\text { I, II } \\
\text { I, II } \\
\text { I, II }\end{array}$ & $\begin{array}{rrr}2 & 6 & 36.465 \\
2 & 5 & 46.158 \\
2 & 3 & 4.581 \\
2 & 0 & 39.978 \\
1 & 59 & 40.327\end{array}$ & $\begin{array}{l}+.005 \\
+.028 \\
+.061 \\
+.032 \\
+.027\end{array}$ & $\begin{array}{l}\mathrm{C} \\
\mathrm{C} \\
\mathrm{C} \\
\mathrm{C} \\
\mathrm{C}\end{array}$ & $\begin{array}{rrr}+11 & 12 & 27.29 \\
+11 & 7 & 47.61 \\
+10 & 52 & 58.31 \\
+10 & 39 & 48.78 \\
+10 & 34 & 24.14\end{array}$ & $\begin{array}{l}+.09 \\
\pm .19 \\
+.31 \\
-.12 \\
-.36\end{array}$ \\
\hline $\begin{array}{l}17.5 \\
22.5 \\
23.5 \\
24.5 \\
26.5\end{array}$ & $\begin{array}{l}\mathrm{P} \\
\mathrm{M} \\
\mathrm{S} \\
\mathrm{Sr} \\
\mathrm{S}\end{array}$ & & $\begin{array}{l}\text { I, II } \\
\text { I, II } \\
\text { I, II } \\
\text { I, II } \\
\text { I, II }\end{array}$ & $\begin{array}{lrr}1 & 58 & 9.300 \\
1 & 55 & 35.010 \\
1 & 55 & 4.020 \\
1 & 54 & 33.049 \\
1 & 53 & 31.142\end{array}$ & $\begin{array}{l}+.050 \\
-.010 \\
+.000 \\
+.029 \\
+.002\end{array}$ & $\begin{array}{l}\mathrm{C} \\
\mathrm{C} \\
\mathrm{C} \\
\mathrm{C} \\
\mathrm{C}\end{array}$ & $\begin{array}{rrr}+10 & 26 & 10.93 \\
+10 & 12 & 19.42 \\
+10 & 9 & 33.71 \\
+10 & 6 & 47.13 \\
+10 & 1 & 15.33\end{array}$ & $\begin{array}{l}-.17 \\
+.12 \\
+.91 \\
+.73 \\
+.23\end{array}$ \\
\hline $\begin{aligned} \text { Nov. } 11.4 \\
15.4\end{aligned}$ & $\begin{array}{l}\mathrm{Sr} \\
\mathrm{M}\end{array}$ & & $\begin{array}{l}. \mathrm{I}, \mathrm{II} \\
\mathrm{I}, \mathrm{II}\end{array}$ & $\begin{array}{llr}1 & 45 & 43.554 \\
1 & 44 & 0.482\end{array}$ & $\begin{array}{l}+.074 \\
+.022\end{array}$ & $\underset{\mathrm{C}}{\mathrm{C}}$ & 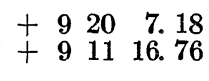 & $\begin{array}{l}+.68 \\
+1.36\end{array}$ \\
\hline $\begin{aligned} & 1918 \\
& \text { Dec. } 17.5 \\
& 19.5\end{aligned}$ & $\underset{\mathrm{P}}{\mathrm{Sr}}$ & & $\begin{array}{l}\text { I, II } \\
\text { I, II }\end{array}$ & $\begin{array}{lll}6 & 55 & 23.335 \\
6 & 54 & 18.478\end{array}$ & $\begin{array}{l}+.025 \\
+.018\end{array}$ & $\underset{\mathrm{C}}{\mathrm{C}}$ & $\begin{array}{r}+2250 \\
+225150.60 \\
+\end{array}$ & $\begin{array}{l}=.82 \\
-.30\end{array}$ \\
\hline $\begin{array}{r}26.5 \\
1919\end{array}$ & M & $W$ & I, II & $\begin{array}{lll}6 & 50 & 22.050\end{array}$ & +.020 & $\mathrm{C}$ & +225745.12 & -.68 \\
\hline 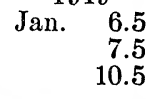 & $\begin{array}{l}\mathrm{Sr} \\
\mathrm{Sr} \\
\mathrm{Sr}\end{array}$ & $\mathrm{E}$ & $\begin{array}{l}\text { I, II } \\
\text { I, II }\end{array}$ & $\begin{array}{lll}6 & 43 & 57.855 \\
6 & 43 & \\
6 & 41 & 40.213\end{array}$ & +.055 & $\begin{array}{l}\mathrm{C} \\
\mathrm{C}\end{array}$ & $\begin{array}{rrrr}+23 & 6 & 31.50 \\
+23 & 7 & 15.73 \\
+23 & 9 & 25.79\end{array}$ & $\begin{array}{l}=.60 \\
=.87 \\
=.31\end{array}$ \\
\hline
\end{tabular}




\begin{tabular}{|c|c|c|c|c|c|c|c|c|}
\hline \multicolumn{9}{|c|}{ J U P I T E R } \\
\hline Date & Obsr. & $\mathrm{Cl}$. & Limb & $\begin{array}{l}\text { App. R. A. } \\
\text { of Center }\end{array}$ & $\begin{array}{l}\text { Corr. to } \\
\text { Am. Eph. }\end{array}$ & Limb & $\begin{array}{l}\text { App. Decl. } \\
\text { of Center }\end{array}$ & $\begin{array}{l}\text { Corr. to } \\
\text { Am. Eph. }\end{array}$ \\
\hline $\begin{array}{cc}1919 \\
\text { Jan. } 13.5 \\
20.4 \\
21.4 \\
24.4 \\
27.4\end{array}$ & $\begin{array}{l}\mathrm{Sr} \\
\mathrm{M} \\
\mathrm{M} \\
\mathrm{P} \\
\mathrm{P}\end{array}$ & $\mathbf{E}$ & $\begin{array}{l}\text { I, II } \\
\text { I, II } \\
\text { I, II } \\
\text { I, II } \\
\text { I, II }\end{array}$ & $\begin{array}{ccc}\mathrm{h} & \mathrm{m} & \mathrm{s} \\
6 & 39 & 59.575 \\
6 & 36 & 16.591 \\
6 & 35 & 46.476 \\
6 & 34 & 18.989 \\
6 & 32 & 56.613\end{array}$ & $\begin{array}{l}s \\
+0.025 \\
-.009 \\
+.026 \\
-.061 \\
-.017\end{array}$ & $\begin{array}{l}\mathrm{C} \\
\mathrm{C} \\
\mathrm{C} \\
\mathrm{C} \\
\mathrm{C}\end{array}$ & \begin{tabular}{rrc}
$\circ$ & \multicolumn{1}{c}{} \\
+23 & 11 & 29.00 \\
+23 & 15 & 49.12 \\
+23 & 16 & 22.82 \\
+23 & 17 & 59.86 \\
+23 & 19 & 28.61
\end{tabular} & $\begin{array}{r}\prime \prime \\
0.00 \\
+\quad .52 \\
+.22 \\
+.56 \\
-.09\end{array}$ \\
\hline $\begin{array}{rr} & 28.4 \\
& 30.4 \\
& 31.4 \\
\text { Feb. } & 3.4 \\
& 6.4\end{array}$ & $\begin{array}{l}\mathrm{P} \\
\mathrm{Sr} \\
\mathrm{Sr} \\
\mathrm{Sr} \\
\mathrm{M}\end{array}$ & $\mathbf{E}$ & $\begin{array}{l}\text { I, II } \\
\text { I, II } \\
\text { I, II } \\
\text { I, II } \\
\text { I, II }\end{array}$ & $\begin{array}{llr}6 & 32 & 30.291 \\
6 & 31 & 39.737 \\
6 & 31 & 15.353 \\
6 & 30 & 6.511 \\
6 & 29 & 4.144\end{array}$ & $\begin{array}{l}+.039 \\
+.067 \\
+.013 \\
+.041 \\
+.004\end{array}$ & $\begin{array}{l}\text { C } \\
\text { C } \\
\mathbf{C} \\
\mathbf{C} \\
\mathbf{C}\end{array}$ & $\begin{array}{rll}+23 & 19 & 56.63 \\
+23 & 20 & 50.46 \\
+23 & 21 & 16.20 \\
+23 & 22 & 29.30 \\
+23 & 23 & 34.42\end{array}$ & $\begin{array}{l}-.37 \\
-.34 \\
-.40 \\
+.30 \\
-.18\end{array}$ \\
\hline $\begin{array}{l}\text { Dec. } 30.6 \\
1920\end{array}$ & M & W & I, II & $9 \begin{array}{lll}9 & 19 & 2.344\end{array}$ & +.044 & $\mathbf{C}$ & +162855.58 & -.82 \\
\hline $\begin{array}{rr}\text { Jan. } & 10.6 \\
12.6 \\
13.6\end{array}$ & $\begin{array}{l}\mathrm{P} \\
\mathrm{P} \\
\mathrm{P}\end{array}$ & & $\begin{array}{l}\text { I, II } \\
\text { I, II } \\
\text { I, II }\end{array}$ & $\begin{array}{llr}9 & 14 & 55.276 \\
9 & 14 & 3.194 \\
9 & 13 & 36.390\end{array}$ & $\begin{array}{l}+.026 \\
+.064 \\
+.040\end{array}$ & $\begin{array}{l}\mathrm{C} \\
\mathrm{C} \\
\mathbf{C}\end{array}$ & 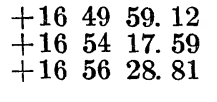 & $\begin{array}{l}-.88 \\
-.31 \\
-.69\end{array}$ \\
\hline $\begin{array}{rr} & 17.6 \\
& 19.6 \\
& 28.5 \\
& 29.5 \\
\text { Feb. } & 2.5\end{array}$ & $\begin{array}{l}\mathrm{P} \\
\mathrm{P} \\
\mathrm{M} \\
\mathrm{M} \\
\mathrm{M}\end{array}$ & & $\begin{array}{l}\text { I, II } \\
\text { I, II } \\
\text { I, II } \\
\text { I, II } \\
\text { I, II }\end{array}$ & $\begin{array}{rrr}9 & 11 & 44.798 \\
9 & 10 & 46.646 \\
9 & 6 & 10.867 \\
9 & 5 & 39.230 \\
9 & 3 & 31.952\end{array}$ & $\begin{array}{l}+.008 \\
+.004 \\
+.047 \\
+.030 \\
+.032\end{array}$ & $\begin{array}{l}\text { C } \\
\mathbf{C} \\
\mathbf{C} \\
\mathbf{C} \\
\mathbf{C}\end{array}$ & 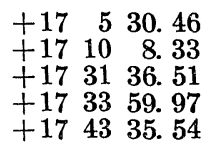 & $\begin{array}{l}-.44 \\
-.97 \\
-.49 \\
-1.43 \\
-.96\end{array}$ \\
\hline $\begin{array}{l}10.5 \\
13.5 \\
17.5 \\
25.4 \\
27.4\end{array}$ & $\begin{array}{l}\mathrm{P} \\
\mathrm{P} \\
\mathrm{P} \\
\mathrm{P} \\
\mathrm{P}\end{array}$ & & $\begin{array}{l}\text { I, II } \\
\text { I, II } \\
\text { I, II } \\
\text { I, II } \\
\text { I, II }\end{array}$ & 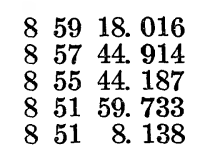 & $\begin{array}{r}+.006 \\
+.004 \\
+.007 \\
+.027 \\
-.012\end{array}$ & $\begin{array}{l}\mathrm{C} \\
\mathbf{C} \\
\mathbf{C} \\
\mathbf{C} \\
\mathrm{C}\end{array}$ & $\begin{array}{rrr}+18 & 2 & 15.21 \\
+18 & 8 & 54.73 \\
+18 & 17 & 26.39 \\
+18 & 32 & 54.07 \\
+18 & 36 & 23.38\end{array}$ & $\begin{array}{l}+.21 \\
-.97 \\
-1.01 \\
-.53 \\
-.02\end{array}$ \\
\hline$\underset{1921}{\text { Mar. }} 3.4$ & $\mathrm{P}$. & W & I, II & $\begin{array}{lll}8 & 49 & 8.617\end{array}$ & +.077 & C & +184419.29 & -.81 \\
\hline $\begin{array}{r}5.6 \\
11.6 \\
12.6\end{array}$ & $\begin{array}{l}\mathrm{P} \\
\mathrm{Ry} \\
\mathrm{M}\end{array}$ & $\mathrm{E}$ & $\begin{array}{l}\text { I, II } \\
\text { I, II } \\
\text { I, II }\end{array}$ & $\begin{array}{rrr}11 & 15 & 0.614 \\
11 & 12 & 43.037 \\
11 & 12 & 18.586\end{array}$ & $\begin{array}{l}+.024 \\
+.027 \\
+.066\end{array}$ & $\begin{array}{l}\mathrm{C} \\
\mathrm{C}\end{array}$ & $\begin{array}{r}+62011.64 \\
+63556.64 \\
+\quad 63841.86\end{array}$ & $\begin{array}{l}-1.06 \\
-.46 \\
-1.14\end{array}$ \\
\hline $\begin{array}{l}14.6 \\
16.6 \\
17.6 \\
18.6 \\
24.5\end{array}$ & $\begin{array}{l}\text { Ry } \\
\text { Ry } \\
\text { P } \\
\text { Bn } \\
\text { Ry }\end{array}$ & & $\begin{array}{l}\text { I, II } \\
\text { I, II } \\
\text { I, II } \\
\text { I, II } \\
\text { I, II }\end{array}$ & $\begin{array}{rrr}11 & 11 & 28.372 \\
11 & 10 & 36.751 \\
11 & 10 & 10.354 \\
11 & 9 & 43.718 \\
11 & 6 & 58.381\end{array}$ & $\begin{array}{l}+.022 \\
+.051 \\
-.026 \\
-.022 \\
-.019\end{array}$ & $\begin{array}{l}\mathrm{C} \\
\mathrm{C} \\
\mathrm{C} \\
\mathrm{C} \\
\mathrm{C}\end{array}$ & 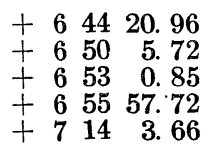 & $\begin{array}{l}-.14 \\
-.98 \\
-1.15 \\
-1.08 \\
-.44\end{array}$ \\
\hline $\begin{aligned} \text { Mar. } 10.5 \\
16.5 \\
18.5 \\
21.5 \\
22.5\end{aligned}$ & $\begin{array}{l}\mathrm{P} \\
\mathrm{Bn} \\
\mathrm{Ry} \\
\mathrm{P} \\
\mathrm{M}\end{array}$ & & $\begin{array}{l}\text { I, II } \\
\text { I, II } \\
\text { I, II } \\
\text { I, II } \\
\text { I, II }\end{array}$ & $\begin{array}{rrr}11 & 0 & 14.856 \\
10 & 57 & 25.047 \\
10 & 56 & 30.119 \\
10 & 55 & 9.903 \\
10 & 54 & 43.754\end{array}$ & $\begin{array}{l}+.076 \\
+.013 \\
+.031 \\
+.023 \\
+.004\end{array}$ & $\begin{array}{l}\mathrm{C} \\
\mathrm{C} \\
\mathrm{C} \\
\mathrm{C} \\
\mathrm{C}\end{array}$ & 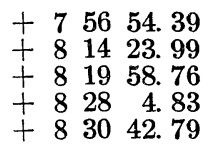 & $\begin{array}{l}-.51 \\
+.09 \\
-.34 \\
-.37 \\
+\quad .19\end{array}$ \\
\hline $\begin{array}{r}23.5 \\
25.4 \\
1922\end{array}$ & $\stackrel{\mathrm{Ry}}{\mathbf{P}}$ & $\mathrm{E}$ & $\begin{array}{l}\text { I, II } \\
\text { I, II }\end{array}$ & $\begin{array}{lll}10 & 54 & 17.943 \\
10 & 53 & 27.469\end{array}$ & $\begin{array}{l}-.017 \\
+.009\end{array}$ & $\stackrel{\mathrm{C}}{\mathrm{C}}$ & $\begin{array}{r}+83316.99 \\
+83818.41\end{array}$ & $\begin{array}{l}-.31 \\
-.19\end{array}$ \\
\hline $\begin{array}{l}\text { Feb. } \quad 28.6 \\
\text { Mar. } \quad 8.6\end{array}$ & $\begin{array}{l}\mathrm{M} \\
\mathrm{Bn}\end{array}$ & $\mathrm{W}$ & $\begin{array}{l}\text { I, II } \\
\text { I, II }\end{array}$ & $\begin{array}{rrr}13 & 8 & 5.824 \\
13 & 5 & 29.826\end{array}$ & $\begin{array}{l}+.034 \\
+.086\end{array}$ & $\stackrel{\mathrm{C}}{\mathrm{C}}$ & $\begin{array}{r}53423.64 \\
-51649.05\end{array}$ & $\begin{array}{l}-1.24 \\
+\quad .25\end{array}$ \\
\hline $\begin{array}{rr} & 13.6 \\
& 15.6 \\
17.6 \\
\\
23.5 \\
\text { Apr. } \quad 12.5\end{array}$ & $\begin{array}{l}\mathrm{M} \\
\mathrm{Ry} \\
\mathrm{Bn} \\
\mathrm{P} \\
\mathrm{Bn}\end{array}$ & $\begin{array}{l}\mathbf{W} \\
\mathbf{E}\end{array}$ & $\begin{array}{l}\text { I, II } \\
\text { I, II } \\
\text { I, II } \\
\text { I, II }\end{array}$ & $\begin{array}{rrr}13 & 3 & 36.166 \\
13 & 2 & \\
13 & 1 & 57.962 \\
12 & 59 & 20.494 \\
12 & 49 & 58.570\end{array}$ & $\begin{array}{r}-.014 \\
+.062 \\
+.054 \\
+.010\end{array}$ & $\begin{array}{l}\mathrm{C} \\
\mathrm{C} \\
\mathrm{C} \\
\mathrm{C} \\
\mathrm{C}\end{array}$ & 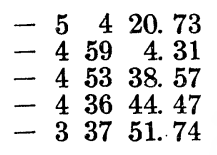 & $\begin{array}{l}-.93 \\
-.91 \\
+.23 \\
-.87 \\
-1.04\end{array}$ \\
\hline $\begin{array}{l}13.5 \\
15.5 \\
18.5 \\
20.5 \\
21.4\end{array}$ & $\begin{array}{l}\mathrm{Bn} \\
\mathrm{Ry} \\
\mathrm{M} \\
\mathrm{Ry} \\
\mathrm{P}\end{array}$ & $\mathbf{E}$ & $\begin{array}{l}\text { I, II } \\
\text { I, II } \\
\text { I, II } \\
\text { I; II } \\
\text { I, II }\end{array}$ & $\begin{array}{lll}12 & 49 & 30.926 \\
12 & 48 & 36.057 \\
12 & 47 & 15.351 \\
12 & 46 & 22.881 \\
12 & 45 & 57.130\end{array}$ & $\begin{array}{l}+.066 \\
+.057 \\
+.011 \\
+.021 \\
+.070\end{array}$ & $\begin{array}{l}\mathrm{C} \\
\mathrm{C} \\
\mathrm{C} \\
\mathrm{C} \\
\mathrm{C}\end{array}$ & 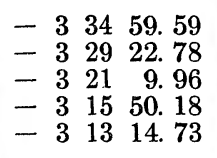 & $\begin{array}{l}+.31 \\
-.18 \\
-.36 \\
+.52 \\
-.23\end{array}$ \\
\hline
\end{tabular}


NINE-INCH TRANSIT CIRCLE OBSERVATIONS, 1913-1926

\begin{tabular}{|c|c|c|c|c|c|c|c|c|}
\hline \multicolumn{9}{|c|}{ J U P I T E R } \\
\hline Date & Obsr. & $\mathrm{Cl}$. & Limb & $\begin{array}{l}\text { App. R. A. } \\
\text { of Center }\end{array}$ & $\begin{array}{l}\text { Corr. to } \\
\text { Am. Eph. }\end{array}$ & Limb & $\begin{array}{l}\text { App. Decl. } \\
\text { of Center }\end{array}$ & $\begin{array}{l}\text { Corr. to } \\
\text { Am. Eph. }\end{array}$ \\
\hline $\begin{array}{cc}1922 \\
\text { Apr. } 22.4 \\
23.4 \\
26.4 \\
1923\end{array}$ & $\begin{array}{l}\mathrm{Bn} \\
\mathrm{Ry} \\
\mathrm{Bn}\end{array}$ & $\mathbf{E}$ & $\begin{array}{l}\text { I, II } \\
\text { I, II } \\
\text { I, II }\end{array}$ & $\begin{array}{ccc}\mathrm{h} & \mathrm{m} & \mathrm{s} \\
12 & 45 & 31.674 \\
12 & 45 & 6.437 \\
12 & 43 & 53.254\end{array}$ & $\begin{array}{l}\text { s } \\
+0.094 \\
+.007 \\
+.064\end{array}$ & $\begin{array}{l}\mathrm{C} \\
\mathrm{C} \\
\mathrm{C}\end{array}$ & 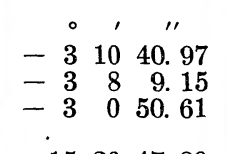 & $\begin{aligned} & \\
&-0.37 \\
&+.05 \\
&-.01\end{aligned}$ \\
\hline Apr. 16.6 & $\mathrm{Bn}$ & $\mathrm{W}$ & I, II & 145658.994 & +.114 & $\mathrm{C}$ & -152647.80 & -.20 \\
\hline $\begin{array}{l}17.6 \\
18.6 \\
20.5 \\
24.5 \\
25.5\end{array}$ & $\begin{array}{l}P \\
\text { Ry } \\
\text { Ry } \\
\text { Ry } \\
\text { Ry }\end{array}$ & & $\begin{array}{l}\text { I, II } \\
\text { I, II } \\
\text { I, II } \\
\text { I, II } \\
\text { I, II }\end{array}$ & $\begin{array}{rrr}14 & 56 & 32.022 \\
14 & 56 & 4.765 \\
14 & 55 & 9.265 \\
14 & 53 & 14.948 \\
14 & 52 & 45.717\end{array}$ & $\begin{array}{l}+.042 \\
+.035 \\
+.025 \\
+.038 \\
+.023\end{array}$ & $\begin{array}{l}\mathrm{C} \\
\mathrm{C} \\
\mathrm{C} \\
\mathrm{C} \\
\mathrm{C}\end{array}$ & $\begin{array}{rrr}-15 & 24 & 50.15 \\
-15 & 22 & 50.90 \\
-15 & 18 & 49.35 \\
-15 & 10 & 29.00 \\
-15 & 8 & 22.13\end{array}$ & $\begin{array}{l}-.25 \\
=.20 \\
-1.15 \\
+.30 \\
-.13\end{array}$ \\
\hline $\begin{array}{r}\text { May } 1.5 \\
2.5 \\
4.5 \\
17.5 \\
19.5\end{array}$ & $\begin{array}{l}\mathrm{P} \\
\mathrm{Ry} \\
\mathrm{P} \\
\mathrm{Ry} \\
\mathrm{Bn}\end{array}$ & $\stackrel{\mathrm{W}}{\mathrm{E}}$ & $\begin{array}{l}\text { I, II } \\
\text { I, II } \\
\text { I, II } \\
\text { I, II } \\
\text { I, II }\end{array}$ & $\begin{array}{lll}14 & 49 & 47.395 \\
14 & 49 & 17.303 \\
14 & 48 & 16.984 \\
14 & 41 & 50.661 \\
14 & 40 & 53.924\end{array}$ & $\begin{array}{l}+.035 \\
+.043 \\
+.064 \\
+.011 \\
+.014\end{array}$ & $\begin{array}{l}\mathrm{C} \\
\mathrm{C} \\
\mathrm{C} \\
\mathrm{C} \\
\mathrm{C}\end{array}$ & $\begin{array}{lll}-14 & 55 & 25.53 \\
-14 & 53 & 14.05 \\
-14 & 48 & 52.54 \\
-14 & 20 & 55.79 \\
-14 & 16 & 51.45\end{array}$ & $\begin{array}{l}-.53 \\
=.05 \\
-1.04 \\
+.31 \\
-.15\end{array}$ \\
\hline $\begin{array}{l}21.4 \\
22.4 \\
24.4 \\
26.4\end{array}$ & $\begin{array}{l}\mathrm{M} \\
\mathrm{P} \\
\mathrm{Bn} \\
\mathrm{Ry}\end{array}$ & & $\begin{array}{l}\text { I, II } \\
\text { I, II } \\
\text { I, II } \\
\text { I, II }\end{array}$ & $\begin{array}{llll}14 & 39 & 58.378 \\
14 & 39 & 31 & 061 \\
14 & 38 & 37.560 \\
14 & 37 & 45.533\end{array}$ & $\begin{array}{l}+.018 \\
+.009 \\
+.020 \\
-.007\end{array}$ & $\begin{array}{l}\mathrm{C} \\
\mathrm{C} \\
\mathrm{C} \\
\mathrm{C}\end{array}$ & $\begin{array}{rrr}-14 & 12 & 52.17 \\
-14 & 10 & 55.20 \\
-14 & 7 & 4.57 \\
-14 & 3 & 22.99\end{array}$ & $\begin{array}{l}-.17 \\
-.60 \\
+.63 \\
+.01\end{array}$ \\
\hline \begin{aligned} \multicolumn{2}{c}{1924} \\
May $\quad 5.6 \\
13.6 \\
16.6 \\
22.5 \\
31.5\end{aligned}$ & $\begin{array}{l}\mathrm{Bn} \\
\mathrm{P} \\
\mathrm{Bn} \\
\mathrm{P} \\
\mathrm{Ry}\end{array}$ & & $\begin{array}{l}\text { I, II } \\
\text { I, II } \\
\text { I, II } \\
\text { I, II } \\
\text { I, II }\end{array}$ & $\begin{array}{rrr}17 & 10 & 20.917 \\
17 & 7 & 2.202 \\
17 & 5 & 39.264 \\
17 & 2 & 42.485 \\
16 & 57 & 57.685\end{array}$ & $\begin{array}{l}+.047 \\
+.082 \\
+.044 \\
+.045 \\
+.075\end{array}$ & $\begin{array}{l}\mathrm{C} \\
\mathrm{C} \\
\mathrm{C} \\
\mathrm{C} \\
\mathrm{C}\end{array}$ & $\begin{array}{rrr}-22 & 14 & 24.30 \\
-22 & 10 & 40.60 \\
-22 & 9 & 3.26 \\
-22 . & 5 & 31.29 \\
-21 & 59 & 34.54\end{array}$ & $\begin{array}{l}+.20 \\
\pm 1.10 \\
-.36 \\
+.11 \\
-.64\end{array}$ \\
\hline 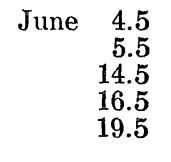 & $\begin{array}{l}\text { Bn } \\
R y \\
R y \\
P \\
R y\end{array}$ & $\begin{array}{l}\mathrm{E} \\
\mathrm{W}\end{array}$ & $\begin{array}{l}\text { I, II } \\
\text { I', II } \\
\text { I, II } \\
\text { I, II }\end{array}$ & $\begin{array}{lll}16 & 56 & \\
16 & 55 & 14.327 \\
16 & 50 & 22.561 \\
16 & 49 & 19.393 \\
16 & 47 & 46.646\end{array}$ & $\begin{array}{l} \pm .027 \\
\pm .009 \\
=.057 \\
-.004\end{array}$ & $\begin{array}{l}\mathrm{C} \\
\mathrm{C} \\
\mathrm{C} \\
\mathrm{C} \\
\mathrm{C}\end{array}$ & $\begin{array}{llll}-21 & 56 & 42.49 \\
-21 & 55 & 59.30 \\
-21 & 49 & 22.93 \\
-21 & 47 & 53.24 \\
-21 & 45 & 42.78\end{array}$ & $\begin{array}{l}+.61 \\
+.30 \\
\pm 1.13 \\
+.26 \\
-.18\end{array}$ \\
\hline $\begin{array}{r} \\
20.5 \\
23.4 \\
25.4 \\
26.4 \\
2 \text { July } \quad 9.4\end{array}$ & $\begin{array}{l}\mathrm{M} \\
\mathrm{Ry} \\
\mathrm{Bn} \\
\mathrm{Bn} \\
\mathrm{Bn}\end{array}$ & & $\begin{array}{l}\text { I, II } \\
\text { I, II } \\
\text { I, II } \\
\text { I, II }\end{array}$ & $\begin{array}{rrr}16 & 47 & 16.276 \\
16 & 45 & 47.315 \\
16 & 44 & 49.837 \\
16 & 44 & \\
16 & 39 & 2.358\end{array}$ & $\begin{array}{l}-.024 \\
+.055 \\
+.057 \\
+.038\end{array}$ & $\begin{array}{l}\mathrm{C} \\
\mathrm{C} \\
\mathrm{C} \\
\mathrm{C} \\
\mathrm{C}\end{array}$ & $\begin{array}{lll}-21 & 44 & 59.56 \\
-21 & 42 & 53.53 \\
-21 & 41 & 29.56 \\
-21 & 40 & 49.20 \\
-21 & 33 & 14.65\end{array}$ & $\begin{array}{l}-.06 \\
-1.23 \\
+.24 \\
+.20 \\
-.05\end{array}$ \\
\hline $\begin{array}{c}11.4 \\
1925\end{array}$ & $\mathbf{M}$ & & I, II & 163821.986 & -.044 & $\mathrm{C}$ & -213219.06 & +.44 \\
\hline 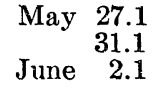 & $\begin{array}{l}\mathrm{Ry} \\
\mathrm{M} \\
\mathrm{M}\end{array}$ & & $\begin{array}{l}\text { I, II } \\
\text { I, II } \\
\text { I, II }\end{array}$ & $\begin{array}{lll}19 & 35 & 29.749 \\
19 & 34 & 31.564 \\
19 & 33 & 58.102\end{array}$ & $\begin{array}{l}-.031 \\
+.014 \\
+.002\end{array}$ & $\begin{array}{l}\mathrm{C} \\
\mathrm{C} \\
\mathrm{C}\end{array}$ & $\begin{array}{l}-214452.85 \\
-21474092 \\
-2149 \\
-25.05\end{array}$ & $\begin{array}{l}-.15 \\
\pm .08 \\
-.55\end{array}$ \\
\hline $\begin{array}{r}4.1 \\
6.1 \\
11.1 \\
12.1 \\
13.1\end{array}$ & $\begin{array}{l}\mathrm{P} \\
\mathrm{P} \\
\mathrm{P} \\
\mathrm{Ry} \\
\mathrm{Ry}\end{array}$ & & $\begin{array}{l}\text { I, II } \\
\text { I, II } \\
\text { I, II } \\
\text { I, II } \\
\text { I, II }\end{array}$ & 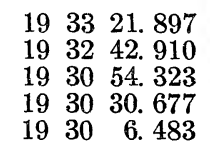 & $\begin{array}{l}+.037 \\
\pm .010 \\
+.053 \\
\pm .013 \\
-.047\end{array}$ & $\begin{array}{l}\mathrm{C} \\
\mathrm{C} \\
\mathrm{C} \\
\mathrm{C} \\
\mathrm{C}\end{array}$ & $\begin{array}{lll}-21 & 50 & 53.42 \\
-21 & 52 & 39.25 \\
-21 & 57 & 22.73 \\
-21 & 58 & 23.57 \\
-21 & 59 & 24.47\end{array}$ & $\begin{array}{l}+.48 \\
\pm .45 \\
+.47 \\
+.07 \\
+.33\end{array}$ \\
\hline $\begin{array}{l}16.1 \\
17.1 \\
20.1 \\
22.1\end{array}$ & $\begin{array}{l}\mathrm{Ry} \\
\stackrel{P}{P} \\
\mathrm{P}\end{array}$ & W & $\begin{array}{l}\text { I, II } \\
\text { I, II } \\
\text { I, II } \\
\text { I, II }\end{array}$ & $\begin{array}{rrr}19 & 28 & 50.621 \\
19 & 28 & 24.266 \\
19 & 27 & 2.146 \\
19 & 26 & 5.150\end{array}$ & $\begin{array}{l}=.029 \\
=.014 \\
-.034 \\
.000\end{array}$ & $\begin{array}{l}\mathrm{C} \\
\mathrm{C} \\
\mathrm{C} \\
\mathrm{C}\end{array}$ & $\begin{array}{llll}-22 & 2 & 35.19 \\
-22 & 3 & 39.00 \\
-22 & 6.59 .35 \\
-22 & 9 & 15.09\end{array}$ & $\begin{array}{l}-.59 \\
+.70 \\
+.35 \\
+.11\end{array}$ \\
\hline
\end{tabular}




\begin{tabular}{|c|c|c|c|c|c|c|c|c|}
\hline \multicolumn{9}{|c|}{$\mathbf{S A T} \mathbf{T} \mathbf{R} \mathbf{N}$} \\
\hline Date & Obsr. & Cl. & $\operatorname{Limb}$ & $\begin{array}{l}\text { App. R. A. } \\
\text { of Center }\end{array}$ & $\begin{array}{l}\text { Corr. to } \\
\text { Am. Eph. }\end{array}$ & $\operatorname{Limb}$ & $\begin{array}{l}\text { App. Decl. } \\
\text { of Center }\end{array}$ & $\begin{array}{l}\text { Corr. to } \\
\text { Am. Eph. }\end{array}$ \\
\hline $\begin{array}{r}1913 \\
\text { Nov. } 13.6 \\
17.6 \\
20.5 \\
21.5 \\
24.5\end{array}$ & $\begin{array}{l}\mathrm{P} \\
\mathrm{Ep} \\
\mathrm{P} \\
\mathrm{P} \\
\mathrm{Ep}\end{array}$ & $\mathbf{E}$ & $\begin{array}{l}\text { I, II } \\
\text { I, II } \\
\text { I, II } \\
\text { I, II } \\
\text { I, II }\end{array}$ & \begin{tabular}{rrr}
$\mathrm{h}$ & $\mathrm{m}$ & \multicolumn{1}{c}{$\mathrm{s}$} \\
5 & 2 & 1.494 \\
5 & 0 & 48.041 \\
4 & 59 & 50.474 \\
4 & 59 & 30.938 \\
4 & 58 & 31.178
\end{tabular} & $\begin{array}{c}\mathrm{s} \\
-0.126 \\
-.059 \\
-.106 \\
-.082 \\
-.112\end{array}$ & $\begin{array}{l}\mathrm{C} \\
\mathrm{C} \\
\mathrm{C} \\
\mathrm{C} \\
\mathrm{C}\end{array}$ & \begin{tabular}{rrr}
$\circ$ & \multicolumn{1}{c}{} \\
+21 & 1 & 36.86 \\
+20 & 59 & 53.34 \\
+20 & 58 & 33.63 \\
+20 & 58 & 6.85 \\
+20 & 56 & 43.54
\end{tabular} & $\begin{array}{l}\text { '" } \\
-0.54 \\
-.16 \\
+.53 \\
+1.05 \\
+\quad .54\end{array}$ \\
\hline Dec. $\begin{array}{r}3.5 \\
9.5 \\
12.5 \\
13.5 \\
18.5\end{array}$ & $\begin{array}{l}\mathrm{P} \\
\mathrm{M} \\
\mathrm{M} \\
\mathrm{Ep} \\
\mathrm{P}\end{array}$ & & $\begin{array}{l}\text { I, II } \\
\text { I, II } \\
\text { I, II } \\
\text { I, II } \\
\text { I, II }\end{array}$ & $\begin{array}{lll}4 & 55 & 25.381 \\
4 & 53 & 19.342 \\
4 & 52 & 16.696 \\
4 & 51 & 55.892 \\
4 & 50 & 13.392\end{array}$ & $\begin{array}{l}-.089 \\
-.088 \\
=.034 \\
-.068 \\
-.168\end{array}$ & $\begin{array}{l}\mathrm{C} \\
\mathrm{C} \\
\mathrm{C} \\
\mathrm{C} \\
\mathrm{C}\end{array}$ & 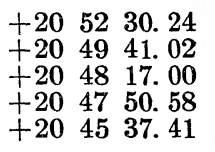 & $\begin{array}{l}+.54 \\
+.12 \\
-1.10 \\
-.22 \\
-.39\end{array}$ \\
\hline $\begin{array}{l}22.4 \\
27.4 \\
29.4 \\
30.4\end{array}$ & $\begin{array}{l}\mathrm{Ep} \\
\mathrm{P} \\
\mathrm{Ep} \\
\mathrm{M}\end{array}$ & & $\begin{array}{l}\text { I, II } \\
\text { I, II } \\
\text { I, II } \\
\text { I, II }\end{array}$ & 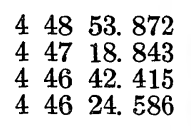 & $\begin{array}{r}-.168 \\
-.057 \\
-.035 \\
-.034\end{array}$ & $\begin{array}{l}\mathrm{C} \\
\mathrm{C} \\
\mathrm{C} \\
\mathrm{C}\end{array}$ & 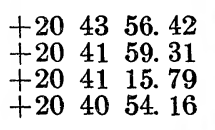 & $\begin{array}{r}-.28 \\
+.21 \\
+.39 \\
-.24\end{array}$ \\
\hline \begin{tabular}{lr}
\multicolumn{2}{c}{1914} \\
Jan. & 6.4 \\
Nov. & 25.6 \\
Dec. & 14.5 \\
& 16.5 \\
& 17.5
\end{tabular} & $\begin{array}{l}M \\
S \\
P \\
S \\
P\end{array}$ & $\stackrel{\mathrm{E}}{\mathrm{W}}$ & $\begin{array}{l}\text { I, I } \\
\text { I, II } \\
\text { I, II } \\
\text { I, II } \\
\text { I, II }\end{array}$ & $\begin{array}{rrr}4 & 44 & 27.921 \\
6 & 3 & 29.094 \\
5 & 57 & 14.972 \\
5 & 56 & 32.440 \\
5 & 56 & 11.128\end{array}$ & $\begin{array}{l}-.019 \\
=.026 \\
=.028 \\
=.090 \\
-.072\end{array}$ & $\begin{array}{l}\mathrm{C} \\
\mathrm{C} \\
\mathrm{C} \\
\mathrm{C} \\
\mathrm{C}\end{array}$ & $\begin{array}{rrr}+20 & 38 & 42.95 \\
+22 & 16 & 43.56 \\
+22 & 17 & 52.39 \\
+22 & 17 & 58.95 \\
+22 & 18 & 2.83\end{array}$ & $\begin{array}{l}-.75 \\
-1.24 \\
=.01 \\
=.55 \\
-.17\end{array}$ \\
\hline $\begin{array}{r}21.5 \\
22.5 \\
30.5 \\
1915\end{array}$ & $\begin{array}{l}\mathrm{P} \\
\mathrm{P} \\
\mathrm{P}\end{array}$ & & $\begin{array}{l}\text { I, II } \\
\text { I, II } \\
\text { I, II }\end{array}$ & $\begin{array}{llll}\mathbf{5} & \mathbf{5 4} & \mathbf{4 5 .} & \mathbf{5 6 6 7} \\
\mathbf{5} & \mathbf{5 4} & \mathbf{2 4} .172 \\
\mathbf{5} & \mathbf{5 1} & \mathbf{3 4 .} & \mathbf{7 3 8}\end{array}$ & $\begin{array}{l}-.073 \\
=.068 \\
-.152\end{array}$ & $\begin{array}{l}\mathrm{C} \\
\mathrm{C} \\
\mathrm{C}\end{array}$ & $\begin{array}{rrr}+22 & 18 & 16.52 \\
+22 & 18 & 20.63 \\
+22 & 18 & 47.34\end{array}$ & $\begin{array}{l}-.28 \\
+.43 \\
+\quad .24\end{array}$ \\
\hline Jan. $\quad 1.5$ & $\mathrm{M}$ & & I, II & $\begin{array}{llll}5 & 50 & 53.462\end{array}$ & -.048 & C & +221853.35 & -.35 \\
\hline $\begin{array}{l}2.5 \\
4.5 \\
5.5 \\
7.4 \\
8.4\end{array}$ & $\begin{array}{l}\mathrm{Sr} \\
\mathrm{Sr} \\
\mathrm{M} \\
\mathrm{S} \\
\mathrm{M}\end{array}$ & & $\begin{array}{l}\text { I, II } \\
\text { I, II } \\
\text { I, II } \\
\text { I, II } \\
\text { I, II }\end{array}$ & $\begin{array}{lll}5 & 50 & 32.915 \\
5 & 49 & 52.415 \\
5 & 49 & 32.474 \\
5 & 48 & 53.018 \\
5 & 48 & 33.654\end{array}$ & $\begin{array}{l}-.105 \\
=.105 \\
=.046 \\
=.082 \\
-.046\end{array}$ & $\begin{array}{l}\mathrm{C} \\
\mathrm{C} \\
\mathrm{C} \\
\mathrm{C} \\
\mathrm{C}\end{array}$ & $\begin{array}{rrr}+22 & 18 & 56.77 \\
+22 & 19 & 3.13 \\
+22 & 19 & 6.80 \\
+22 & 19 & 13.62 \\
+22 & 19 & 16.87\end{array}$ & $\begin{array}{l}-.33 \\
=.67 \\
=.40 \\
-.38 \\
-.63\end{array}$ \\
\hline $\begin{array}{r}9.4 \\
26.4 \\
\text { Dec. } 8.6 \\
9.6 \\
10.6\end{array}$ & $\begin{array}{l}\mathrm{S} \\
\mathrm{M} \\
\mathrm{M} \\
\mathrm{P} \\
\mathrm{S}\end{array}$ & $\begin{array}{l}\mathrm{W} \\
\mathrm{E}\end{array}$ & $\begin{array}{l}\text { I, II } \\
\text { I, II } \\
\text { I, II } \\
\text { I, II } \\
\text { I, II }\end{array}$ & $\begin{array}{rrrr}5 & 48 & 14.424 \\
5 & 43 & 32.066 \\
7 & 5 & 10.196 \\
7 & 4 & 52.992 \\
7 & 4 & 35.521\end{array}$ & $\begin{array}{l}-.096 \\
=.064 \\
=.034 \\
=.038 \\
-.009\end{array}$ & $\begin{array}{l}\mathrm{C} \\
\mathrm{C} \\
\mathrm{C} \\
\mathrm{C} \\
\mathrm{C}\end{array}$ & $\begin{array}{rrr}+22 & 19 & 20.86 \\
+22 & 20 & 33.97 \\
+22 & 4 & 46.73 \\
+22 & 5 & 17.95 \\
+22 & 5 & 50.19\end{array}$ & $\begin{array}{l}-.14 \\
=.83 \\
=.37 \\
=.45 \\
-.01\end{array}$ \\
\hline $\begin{array}{l}21.5 \\
22.5 \\
23.5 \\
24.5 \\
31.5\end{array}$ & $\begin{array}{l}\mathrm{Sr} \\
\mathrm{P} \\
\mathrm{Sr} \\
\mathrm{P} \\
\mathrm{S}\end{array}$ & & $\begin{array}{l}\text { I, II } \\
\text { I, II } \\
\text { I, II } \\
\text { I, II }\end{array}$ & $\begin{array}{rrr}7 & 1 & 6.795 \\
7 & 0 & 46.670 \\
7 & 0 & 26.295 \\
7 & 0 & 5.892 \\
6 & 58 & \end{array}$ & $\begin{array}{l}-.085 \\
-.040 \\
-.095 \\
-.028\end{array}$ & $\begin{array}{l}\mathrm{C} \\
\mathrm{C} \\
\mathrm{C} \\
\mathrm{C} \\
\mathrm{C}\end{array}$ & $\begin{array}{rrr}+22 & 12 & 0.35 \\
+22 & 12 & 34.82 \\
+22 & 13 & 9.24 \\
+22 & 13 & 45.06 \\
+22 & 17 & 53.67\end{array}$ & $\begin{array}{l}+.25 \\
-.28 \\
-.96 \\
-.34 \\
+.07\end{array}$ \\
\hline \begin{tabular}{rr}
\multicolumn{2}{c}{1916} \\
Jan. $\quad 2.5$ \\
3.5 \\
8.5 \\
14.5
\end{tabular} & $\begin{array}{l}\mathrm{S} \\
\mathrm{P} \\
\mathrm{P} \\
\mathrm{Sr}\end{array}$ & & $\begin{array}{l}\text { I, II } \\
\text { I, II } \\
\text { I, II } \\
\text { I, II }\end{array}$ & $\begin{array}{lll}6 & 56 & 57.069 \\
6 & 56 & 35.864 \\
6 & 54 & 49.640 \\
6 & 52 & 44.251\end{array}$ & $\begin{array}{l}-.101 \\
-.056 \\
-.130 \\
-.089\end{array}$ & $\begin{array}{l}\mathrm{C} \\
\stackrel{\mathrm{C}}{\mathrm{C}}\end{array}$ & 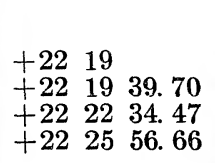 & $\begin{array}{l}-.10 \\
-.13 \\
-1.54\end{array}$ \\
\hline $\begin{array}{rr} & 23.4 \\
& 24.4 \\
& 25.4 \\
\text { Feb. } & 3.4 \\
& 7.4 \\
\text { Dec. } & 17.6 \\
& 22.6 \\
& 25.6\end{array}$ & $\begin{array}{l}M \\
S \\
\mathrm{P} \\
\mathrm{S} \\
\mathrm{S} \\
\mathrm{M} \\
\mathrm{S} \\
\mathrm{Sr}\end{array}$ & $\begin{array}{l}\mathrm{E} \\
\mathrm{W} \\
\mathrm{W} \\
\mathrm{E} \\
\mathrm{E}\end{array}$ & $\begin{array}{l}\text { I, II } \\
\text { I, II } \\
\text { I, II } \\
\text { I, II } \\
\text { I, II } \\
\text { I, II } \\
\text { I, II } \\
\text { I, II }\end{array}$ & $\begin{array}{rrr}6 & 49 & 45.440 \\
6 & 49 & 26.564 \\
6 & 49 & 7.887 \\
6 & 46 & 32.463 \\
6 & 45 & 31.676 \\
8 & 6 & 30.536 \\
8 & 5 & 10.554 \\
8 & 4 & 19.220\end{array}$ & $\begin{array}{r}-.120 \\
=.116 \\
=.143 \\
-.107 \\
-.104 \\
-.084 \\
-.146 \\
.000\end{array}$ & $\begin{array}{l}\mathrm{C} \\
\mathrm{C} \\
\mathrm{C} \\
\mathrm{C} \\
\mathrm{C} \\
\mathrm{C} \\
\mathrm{C} \\
\mathrm{C}\end{array}$ & $\begin{array}{rrr}+22 & 30 & 44.77 \\
+22 & 31 & 14.39 \\
+22 & 31 & 44.26 \\
+22 & 35 & 53.15 \\
+22 & 37 & 32.79 \\
+20 & 25 & 14.61 \\
+20 & 29 & 48.29 \\
+20 & 32 & 41.69\end{array}$ & $\begin{array}{l}+.57 \\
+.09 \\
+.36 \\
+.25 \\
-.21 \\
+.21 \\
-.01 \\
-.31\end{array}$ \\
\hline \begin{tabular}{l}
\multicolumn{2}{c}{1917} \\
Jan. $\quad 6.5$
\end{tabular} & $\mathrm{P}$ & W & I, II & $\begin{array}{lll}8 & 0 & 32.959\end{array}$ & -.051 & $\mathrm{C}$ & $+2045 \quad 3.51$ & +.81 \\
\hline
\end{tabular}




\begin{tabular}{|c|c|c|c|c|c|c|c|c|}
\hline \multicolumn{9}{|c|}{$\mathbf{S A T} \mathbf{A} \mathbf{R}$} \\
\hline Date & Obsr. & Cl. & Limb & $\begin{array}{l}\text { App. R A. } \\
\text { of Center }\end{array}$ & $\begin{array}{l}\text { Corr. to } \\
\text { Am. Eph. }\end{array}$ & Limb & $\begin{array}{l}\text { App. Decl. } \\
\text { of Center }\end{array}$ & $\begin{array}{l}\text { Corr. to } \\
\text { Am. Eph. }\end{array}$ \\
\hline 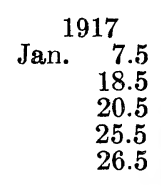 & $\begin{array}{l}\mathrm{M} \\
\mathrm{P} \\
\mathrm{P} \\
\mathrm{S} \\
\mathrm{Sr}\end{array}$ & W & $\begin{array}{l}\text { I, II } \\
\text { I, II } \\
\text { I, II } \\
\text { I, II } \\
\text { I, II }\end{array}$ & $\begin{array}{ccc}\mathrm{h} & \mathrm{m} & \mathrm{s} \\
8 & 0 & 12.951 \\
7 & 56 & 28.120 \\
7 & 55 & 46.976 \\
7 & 54 & 4.717 \\
7 & 53 & 44.595\end{array}$ & $\begin{aligned} & s \\
&- 0.099 \\
&= .160 \\
&= .084 \\
&=.133 \\
&-.055\end{aligned}$ & $\begin{array}{l}\mathrm{C} \\
\mathrm{C} \\
\mathrm{C} \\
\mathrm{C} \\
\mathrm{C}\end{array}$ & $\begin{array}{rcc}\circ & 1 & \prime \prime \\
+20 & 46 & 6.51 \\
+20 & 57 & 52.50 \\
+20 & 59 & 58.17 \\
+21 & 5 & 10.00 \\
+21 & 6 & 10.71\end{array}$ & $\begin{array}{r}\prime \prime \\
-0.09 \\
+.80 \\
+.23 \\
+.50 \\
+.21\end{array}$ \\
\hline $\begin{array}{r}28.5 \\
30.5 \\
\text { Feb. } 6.4 \\
7.4 \\
14.4\end{array}$ & $\begin{array}{l}\mathrm{M} \\
\mathrm{Sr} \\
\mathrm{P} \\
\mathrm{Sr} \\
\mathrm{P}\end{array}$ & & $\begin{array}{l}\text { I, II } \\
\text { I, II } \\
\text { I, II } \\
\text { I, II } \\
\text { I, II }\end{array}$ & $\begin{array}{rrr}7 & 53 & 4.472 \\
7 & 52 & 24.994 \\
7 & 50 & 12.096 \\
7 & 49 & 53.875 \\
7 & 47 & 53.870\end{array}$ & $\begin{array}{l}=.128 \\
=.076 \\
=.044 \\
=.105 \\
=.130\end{array}$ & $\begin{array}{l}\mathrm{C} \\
\mathrm{C} \\
\mathrm{C} \\
\mathrm{C} \\
\mathrm{C}\end{array}$ & $\begin{array}{rrr}+21 & 810.65 \\
+21 & 109.25 \\
+21 & 16 & 42.77 \\
+21 & 17 & 35.26 \\
+21 & 23 & 26.02\end{array}$ & $\begin{array}{l}-.25 \\
+.15 \\
+.27 \\
+.44 \\
+.12\end{array}$ \\
\hline 1918 & $P$ & & I, II & $\begin{array}{lll}747 & 6.868\end{array}$ & -.052 & $\mathrm{C}$ & +212542.80 & +.10 \\
\hline $\begin{array}{lr}\text { Jan. } & 25.5 \\
\text { Feb. } & 9.5 \\
& 11.5\end{array}$ & $\begin{array}{l}\mathrm{Sr} \\
\mathrm{M} \\
\mathrm{P}\end{array}$ & & $\begin{array}{l}\text { I, II } \\
\text { I, II } \\
\text { I, II }\end{array}$ & $\begin{array}{lll}8 & 57 & 3.156 \\
8 & 52 & 9.264 \\
8 & 51 & 30.946\end{array}$ & $\begin{array}{l}=.184 \\
=.136 \\
=.084\end{array}$ & $\begin{array}{l}\mathrm{C} \\
\mathrm{C} \\
\mathrm{C}\end{array}$ & $\begin{array}{rrr}+18 & 859.46 \\
+1830 & 27.47 \\
+18 & 33 & 11.18\end{array}$ & $\begin{array}{l}=.34 \\
=.13 \\
-.22\end{array}$ \\
\hline $\begin{array}{l}12.5 \\
13.5 \\
15.5 \\
18.5 \\
20.5\end{array}$ & $\begin{array}{l}\mathrm{Sr} \\
\mathrm{M} \\
\mathrm{Sr} \\
\mathrm{M} \\
\mathrm{M}\end{array}$ & & $\begin{array}{l}\text { I, II } \\
\text { I, II } \\
\text { I, II } \\
\text { I' II } \\
\text { I, II }\end{array}$ & 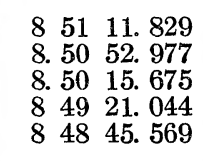 & $\begin{array}{l}=.191 \\
=.163 \\
=: 175 \\
=: 106 \\
=.101\end{array}$ & $\begin{array}{l}\mathrm{C} \\
\mathrm{C} \\
\mathrm{C} \\
\mathrm{C} \\
\mathrm{C}\end{array}$ & 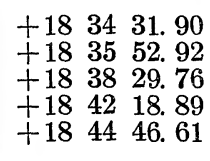 & $\begin{array}{l}-.30 \\
+.82 \\
+.16 \\
+.19 \\
+.11\end{array}$ \\
\hline $\begin{array}{rr}21.4 \\
23.4 \\
28.4 \\
\text { Mar. } 1.4 \\
\quad 2.4\end{array}$ & $\begin{array}{l}\mathrm{Sr} \\
\mathrm{M} \\
\mathrm{Sr} \\
\mathrm{P} \\
\mathrm{M}\end{array}$ & & $\begin{array}{l}\text { I, II } \\
\text { I, II } \\
\text { I, II } \\
\text { I, II } \\
\text { I, II }\end{array}$ & $\begin{array}{lrr}8 & 48 & 28.167 \\
8 & 47 & 53.964 \\
8 & 46 & 32.919 \\
8 & 46 & 17.428 \\
8 & 46 & 2.412\end{array}$ & $\begin{array}{l}=.083 \\
=.106 \\
=.111 \\
=.212 \\
=.128\end{array}$ & $\begin{array}{l}\mathrm{C} \\
\mathbf{C} \\
\mathbf{C} \\
\mathbf{C} \\
\mathbf{C}\end{array}$ & 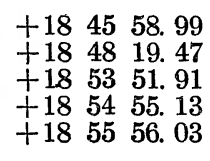 & $\begin{array}{l}+.19 \\
\pm .53 \\
+.01 \\
+.73 \\
+.53\end{array}$ \\
\hline $\begin{array}{r}5.4 \\
7.4\end{array}$ & $\stackrel{\mathrm{P}}{\mathrm{S} r}$ & $\mathrm{~W}$ & $\begin{array}{l}\text { I, II } \\
\text { I, II }\end{array}$ & $\begin{array}{lll}8 & 45 & 19.017 \\
8 & 44 & 51.562\end{array}$ & $\begin{array}{l}-.053 \\
-.128\end{array}$ & $\stackrel{\mathrm{C}}{\mathrm{C}}$ & $\begin{array}{r}+185850.68 \\
+190 \\
+10.76\end{array}$ & $\begin{array}{l}-.32 \\
-.24\end{array}$ \\
\hline 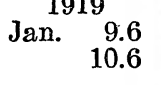 & $\stackrel{\mathrm{P}}{\mathrm{P}}$ & $\mathrm{E}$ & $\begin{array}{l}\text { I, II } \\
\text { I, II }\end{array}$ & $\begin{array}{rrr}10 & 0 & 13.310 \\
10 & 0 & 0.846\end{array}$ & $\begin{array}{l}-.050 \\
-.114\end{array}$ & $\stackrel{\mathrm{C}}{\mathrm{C}}$ & $\begin{array}{r}+134134.50 \\
+134252.12\end{array}$ & $\begin{array}{l}+.80 \\
-.08\end{array}$ \\
\hline $\begin{array}{l}13.6 \\
20.6 \\
21.6 \\
24.6 \\
27.6\end{array}$ & $\begin{array}{l}\mathrm{M} \\
\mathrm{P} \\
\mathrm{M} \\
\mathrm{M} \\
\mathrm{Sr}\end{array}$ & & $\begin{array}{l}\text { I, II } \\
\text { I, II } \\
\text { I, II } \\
\text { I, II } \\
\text { I, II }\end{array}$ & $\begin{array}{lll}9 & 59 & 21.783 \\
9 & 57 & 40.160 \\
9 & 57 & 24.682 \\
9 & 56 & 36.400 \\
9 & 55 & 46.277\end{array}$ & $\begin{array}{l}=.077 \\
=.120 \\
=.008 \\
=.110 \\
=.093\end{array}$ & $\begin{array}{l}\mathrm{C} \\
\mathrm{C} \\
\mathrm{C} \\
\mathrm{C} \\
\mathrm{C}\end{array}$ & $\begin{array}{rrr}+13 & 46 & 57.89 \\
+13 & 57 & 16.88 \\
+13 & 58 & 50.26 \\
+14 & 3 & 35.22 \\
+14 & 8 & 29.36\end{array}$ & $\begin{array}{l}+.89 \\
+.78 \\
+.96 \\
+.12 \\
+.36\end{array}$ \\
\hline $\begin{array}{lr} & 28.6 \\
& 30.6 \\
\text { Feb. } & 24.5 \\
& 27.5 \\
\text { Mar. } \quad 3.5\end{array}$ & $\begin{array}{l}\mathrm{P} \\
\mathrm{P} \\
\mathrm{Sr} \\
\mathrm{M} \\
\mathrm{M}\end{array}$ & & $\begin{array}{l}\text { I, II } \\
\text { I, II } \\
\text { I, II } \\
\text { I, II } \\
\text { I, II }\end{array}$ & $\begin{array}{lrrr}9 & 55 & 29.160 \\
9 & 54 & 54.406 \\
9 & 47 & 13.159 \\
9 & 46 & 18.856 \\
9 & 45 & 8.377\end{array}$ & $\begin{array}{l}=.100 \\
=.094 \\
=.131 \\
=.134 \\
=.113\end{array}$ & $\begin{array}{l}\mathrm{C} \\
\mathrm{C} \\
\mathrm{C} \\
\mathrm{C} \\
\mathrm{C}\end{array}$ & $\begin{array}{rrr}+14 & 10 & 9.31 \\
+14 & 13 & 30.33 \\
+14 & 55 & 58.37 \\
+15 & 0 & 45.47 \\
+15 & 6 & 54.14\end{array}$ & $\begin{array}{l}+.71 \\
+.73 \\
+.57 \\
+.67 \\
+.64\end{array}$ \\
\hline $\begin{array}{r}4.5 \\
11.4 \\
18.4 \\
21.4\end{array}$ & $\begin{array}{l}\mathrm{M} \\
\mathrm{P} \\
\mathrm{Sr} \\
\mathrm{Sr}\end{array}$ & $\mathbf{E}$ & $\begin{array}{l}\text { I, II } \\
\text { I, II } \\
\text { I, II } \\
\text { I, II }\end{array}$ & $\begin{array}{lll}9 & 44 & 51.157 \\
9 & 42 & 56.419 \\
9 & 41 & 13.603 \\
9 & 40 & 33.887\end{array}$ & $\begin{array}{l}=.123 \\
=.071 \\
=.107 \\
=.073\end{array}$ & $\begin{array}{l}\mathrm{C} \\
\mathrm{C} \\
\mathrm{C} \\
\mathrm{C}\end{array}$ & $\begin{array}{rrr}+15 & 823.38 \\
+15 & 18 & 11.21 \\
+15 & 26 & 46.05 \\
+15 & 30 & 1.22\end{array}$ & $\begin{array}{l}+.68 \\
+.51 \\
+.65 \\
+.32\end{array}$ \\
\hline \begin{tabular}{cc}
\multicolumn{2}{c}{1920} \\
Jan. 28.6 \\
& 29.6 \\
Feb. & 2.6 \\
& 11.6 \\
& 19.5
\end{tabular} & $\begin{array}{l}\mathrm{M} \\
\mathrm{M} \\
\mathrm{M} \\
\mathrm{P} \\
\mathrm{M}\end{array}$ & $\mathrm{W}$ & $\begin{array}{l}\text { I, II } \\
\text { I, II } \\
\text { I, II } \\
\text { I, II } \\
\text { I, II }\end{array}$ & $\begin{array}{rrr}10 & 50 & 52.950 \\
10 & 50 & 39.506 \\
10 & 49 & 42.933 \\
10 & 47 & 22.056 \\
10 & 45 & 5.244\end{array}$ & $\begin{array}{l}=.130 \\
=.084 \\
=.047 \\
=.104 \\
=.096\end{array}$ & $\begin{array}{l}\mathrm{C} \\
\mathrm{C} \\
\mathrm{C} \\
\mathrm{C} \\
\mathrm{C}\end{array}$ & $\begin{array}{rrr}+9 & 24 & 23.93 \\
+9 & 25 & 57.15 \\
+9 & 32 & 29.05 \\
+9 & 48 & 16.71 \\
+10 & 3 & 7.55\end{array}$ & $\begin{array}{l}+1.03 \\
\pm .35 \\
+.15 \\
+.41 \\
+.65\end{array}$ \\
\hline $\begin{array}{rr} & 25.5 \\
\text { Mar. } & 1.5 \\
3.5 \\
8.5 \\
9.5\end{array}$ & $\begin{array}{l}\mathrm{P} \\
\mathrm{P} \\
\mathrm{P} \\
\mathrm{M} \\
\mathrm{P}\end{array}$ & W & $\begin{array}{l}\text { I, II } \\
\text { I, II } \\
\text { I, II } \\
\text { I, II } \\
\text { I, II }\end{array}$ & $\begin{array}{lll}10 & 43 & 18.529 \\
10 & 41 & 48.803 \\
10 & 41 & 13.009 \\
10 & 39 & 44.274 \\
10 & 39 & 26.817\end{array}$ & $\begin{array}{l}=.121 \\
=.077 \\
=.051 \\
=.136 \\
=.083\end{array}$ & $\begin{array}{l}\mathrm{C} \\
\mathrm{C} \\
\mathrm{C} \\
\mathrm{C} \\
\mathrm{C}\end{array}$ & $\begin{array}{rrr}+10 & 14 & 25.19 \\
+10 & 23 & 45.86 \\
+10 & 27 & 26.72 \\
+10 & 36 & 28.55 \\
+10 & 38 & 14.08\end{array}$ & $\begin{array}{l}+.19 \\
+.56 \\
+.22 \\
+.75 \\
+.38\end{array}$ \\
\hline
\end{tabular}




\begin{tabular}{|c|c|c|c|c|c|c|c|c|}
\hline \multicolumn{9}{|c|}{$\mathbf{S A T} \mathbf{T} \mathbf{T} \mathbf{N}$} \\
\hline Date & Obsr. & Cl. & Limb & $\begin{array}{l}\text { App. R. A. } \\
\text { of Center }\end{array}$ & $\begin{array}{l}\text { Corr. to } \\
\text { Am. Eph. }\end{array}$ & $\operatorname{Limb}$ & $\begin{array}{l}\text { App. Decl. } \\
\text { of Center }\end{array}$ & $\begin{array}{l}\text { Corr. to } \\
\text { Am. Eph. }\end{array}$ \\
\hline $\begin{array}{c}1920 \\
\text { Mar. } 22.4 \\
23.4 \\
24.4 \\
25.4 \\
27.4\end{array}$ & $\begin{array}{l}\mathrm{Bn} \\
\mathrm{P} \\
\mathrm{M} \\
\mathrm{Bn} \\
\mathrm{Bn}\end{array}$ & W & $\begin{array}{l}\text { I, II } \\
\text { I, II } \\
\text { I, II } \\
\text { I, II } \\
\text { I, II }\end{array}$ & $\begin{array}{rrr}\mathrm{h} & \mathrm{m} & \mathrm{s} \\
10 & 35 & 51.429 \\
10 & 35 & 36.272 \\
10 & 35 & 21.241 \\
10 & 35 & 6.340 \\
10 & 34 & 37.428\end{array}$ & $\begin{array}{l}s \\
-0.231 \\
-.108 \\
-.079 \\
-.160 \\
-.182\end{array}$ & $\begin{array}{l}\mathrm{C} \\
\mathrm{C} \\
\mathrm{C} \\
\mathrm{C} \\
\mathrm{C}\end{array}$ & \begin{tabular}{rrc}
$\circ$ & \multicolumn{1}{c}{} & \\
+10 & 59 & 26.58 \\
+11 & 0 & 54.78 \\
+11 & 2 & 20.39 \\
+11 & 38 \\
+11 & 3 & 44.91 \\
+11 & 6 & 29.60
\end{tabular} & 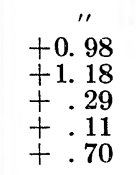 \\
\hline \begin{aligned} & \multicolumn{2}{c}{1921} \\
& Feb. 12.6 \\
& 14.6 \\
& 16.6 \\
& 17.6\end{aligned} & $\begin{array}{l}M \\
R y \\
R y \\
P\end{array}$ & $\mathbf{E}$ & $\begin{array}{l}\text { I, II } \\
\text { I, II } \\
\text { I, II } \\
\text { I, II }\end{array}$ & $\begin{array}{rrr}11 & 39 & 35.482 \\
11 & 39 & 7.576 \\
11 & 38 & 38.817 \\
11 & 38 & 24.038\end{array}$ & $\begin{array}{l}-.088 \\
-.154 \\
-.123 \\
-.172\end{array}$ & $\begin{array}{l}\mathrm{C} \\
\mathrm{C} \\
\mathrm{C} \\
\mathrm{C}\end{array}$ & $\begin{array}{r}+44342.35 \\
+447 \\
+4.61 \\
+45035.58 \\
+45222.05\end{array}$ & $\begin{array}{l}-.25 \\
+1.01 \\
+.08 \\
+.45\end{array}$ \\
\hline $\begin{array}{r}18.6 \\
24.6 \\
\text { Mar. } 10.5 \\
16.5 \\
18.5\end{array}$ & $\begin{array}{l}\mathrm{Bn} \\
\mathrm{Ry} \\
\mathrm{P} \\
\mathrm{Bn} \\
\mathrm{Ry}\end{array}$ & & $\begin{array}{l}\text { I, II } \\
\text { I, II } \\
\text { I, II } \\
\text { I, II } \\
\text { I, II }\end{array}$ & $\begin{array}{rrr}11 & 38 & 9.109 \\
11 & 36 & 35.530 \\
11 & 32 & 38.750 \\
11 & 30 & 54.209 \\
11 & 30 & 19.686\end{array}$ & $\begin{array}{l}-.161 \\
-.110 \\
-.090 \\
-.191 \\
-.104\end{array}$ & $\begin{array}{l}\mathrm{C} \\
\mathrm{C} \\
\mathrm{C} \\
\mathrm{C} \\
\mathrm{C}\end{array}$ & 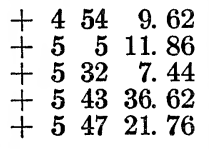 & $\begin{array}{l}+.82 \\
+.56 \\
+.54 \\
+.42 \\
+.16\end{array}$ \\
\hline $\begin{array}{rr} & 21.5 \\
& 23.5 \\
& 25.5 \\
\text { Apr. } \quad 5.4 \\
& 6.4\end{array}$ & $\begin{array}{l}\mathrm{P} \\
\mathrm{Ry} \\
\mathbf{P} \\
\mathrm{M} \\
\mathrm{Ry}\end{array}$ & $\begin{array}{l}E \\
W\end{array}$ & $\begin{array}{l}\text { I, II } \\
\text { I, II } \\
\text { I, II } \\
\text { I, II } \\
\text { I, II }\end{array}$ & $\begin{array}{lll}11 & 29 & 28.202 \\
11 & 28 & 54.293 \\
11 & 28 & 20.791 \\
11 & 25 & 26.632 \\
11 & 25 & 11.875\end{array}$ & $\begin{array}{l}-.118 \\
-.117 \\
-.089 \\
-.118 \\
-.135\end{array}$ & $\begin{array}{l}\mathrm{C} \\
\mathrm{C} \\
\mathrm{C} \\
\mathrm{C} \\
\mathrm{C}\end{array}$ & 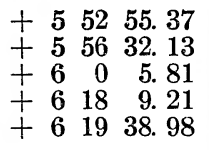 & $\begin{array}{l}+.97 \\
+.23 \\
+.51 \\
+.11 \\
+.28\end{array}$ \\
\hline $1922^{9.4}$ & $\mathrm{Bn}$ & & I, II & 112428.964 & -.166 & $\mathrm{C}$ & +62357.56 & +.66 \\
\hline $\begin{array}{lr}\text { Feb. } & 28.6 \\
\text { Mar. } & 8.6 \\
& 13.5\end{array}$ & $\begin{array}{l}\mathrm{M} \\
\mathrm{Bn} \\
\mathrm{M}\end{array}$ & & $\begin{array}{l}\text { I, II } \\
\text { I, II } \\
\text { I, II }\end{array}$ & $\begin{array}{rrr}12 & 26 & 21.755 \\
12 & 24 & 21.748 \\
12 & 23 & 1.376\end{array}$ & $\begin{array}{l}-.145 \\
-.142 \\
-.134\end{array}$ & $\begin{array}{l}\mathrm{C} \\
\mathrm{C} \\
\mathrm{C}\end{array}$ & $\begin{array}{rrrr}- & 0 & 1 & 52.46 \\
+ & 0 & 12 & 21.88 \\
+ & 0 & 21 & 39.19\end{array}$ & 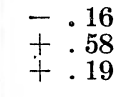 \\
\hline $\begin{array}{rr} & 15.5 \\
& 17.5 \\
& 23.5 \\
\text { Apr. } & 11.5 \\
12.5\end{array}$ & $\begin{array}{l}\mathrm{Bn} \\
\mathrm{M} \\
\mathrm{P} \\
\mathrm{P} \\
\mathrm{Bn}\end{array}$ & $\begin{array}{l}\mathrm{W} \\
\mathrm{E}\end{array}$ & $\begin{array}{l}\text { I, II } \\
\text { I, II } \\
\text { I, II } \\
\text { I, II } \\
\text { I, II }\end{array}$ & $\begin{array}{lll}12 & 22 & 28.311 \\
12 & 21 & 54.944 \\
12 & 20 & 13.154 \\
12 & 14 & 54.490 \\
12 & 14 & 38.696\end{array}$ & $\begin{array}{l}-.189 \\
-.136 \\
-.056 \\
-.140 \\
-.124\end{array}$ & $\begin{array}{l}\mathrm{C} \\
\mathrm{C} \\
\mathrm{C} \\
\mathrm{C} \\
\mathrm{C}\end{array}$ & 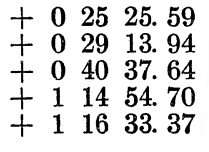 & $\begin{array}{l}+.19 \\
+1.14 \\
+.34 \\
+1.40 \\
+1.57\end{array}$ \\
\hline $\begin{array}{l}13.5 \\
15.4 \\
18.4 \\
20.4 \\
21.4 \\
22.4 \\
23.4\end{array}$ & $\begin{array}{l}\mathrm{Bn} \\
\mathrm{Ry} \\
\mathrm{M} \\
\mathrm{Ry} \\
\mathrm{P} \\
\mathrm{Bn} \\
\mathrm{Ry}\end{array}$ & $\stackrel{\mathrm{E}}{\mathrm{W}}$ & $\begin{array}{l}\text { I, II } \\
\text { I, II } \\
\text { I, II } \\
\text { I, II } \\
\text { I, II } \\
\text { I, II } \\
\text { I, II }\end{array}$ & 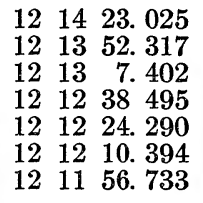 & $\begin{array}{l}-.145 \\
-.053 \\
-. .108 \\
-.085 \\
=.150 \\
=.136 \\
-.107\end{array}$ & $\begin{array}{l}\mathrm{C} \\
\mathrm{C} \\
\mathrm{C} \\
\mathrm{C} \\
\mathrm{C} \\
\mathrm{C} \\
\mathrm{C}\end{array}$ & 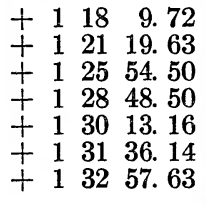 & $\begin{array}{l}+.82 \\
+\quad .63 \\
+1.60 \\
+1.00 \\
+.86 \\
+.74 \\
+\quad .93\end{array}$ \\
\hline $\begin{aligned} 1923 \\
\text { Mar. } 14.6 \\
17.6\end{aligned}$ & $\begin{array}{l}\mathrm{Ry} \\
\mathrm{P}\end{array}$ & & $\begin{array}{l}\text { I, II } \\
\text { I, II }\end{array}$ & 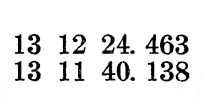 & $\begin{array}{l}-.077 \\
-.052\end{array}$ & $\begin{array}{l}\mathrm{C} \\
\mathrm{C}\end{array}$ & $\begin{array}{r}-44341.34 \\
-43843.49\end{array}$ & $\begin{array}{l}+.56 \\
-.09\end{array}$ \\
\hline $\begin{array}{r}20.6 \\
28.5 \\
29.5 \\
\text { Apr. } 9.5 \\
10.5\end{array}$ & $\begin{array}{l}\mathrm{P} \\
\mathrm{Ry} \\
\mathrm{P} \\
\mathrm{Ry} \\
\mathrm{P}\end{array}$ & & $\begin{array}{l}\text { I, II } \\
\text { I, II }\end{array}$ & $\begin{array}{rrr}13 & 10 & 54.006 \\
13 & 8 & 44.753 \\
13 & 8 & \\
13 & 5 & 21.141 \\
13 & 5 & 4.056\end{array}$ & $\begin{array}{l}-.124 \\
-.077 \\
-.109 \\
-.104\end{array}$ & $\begin{array}{l}\mathrm{C} \\
\mathrm{C} \\
\mathrm{C} \\
\mathrm{C} \\
\mathrm{C}\end{array}$ & $\begin{array}{rrr}-4 & 33 & 37.30 \\
-4 & 19 & 34.23 \\
-4 & 17 & 47.81 \\
- & 358 & 9.40 \\
- & 356 & 24.25\end{array}$ & $\begin{array}{r}.00 \\
+.17 \\
+.41 \\
+.50 \\
+.05\end{array}$ \\
\hline $\begin{array}{l}16.5 \\
18.5 \\
19.5 \\
20.5 \\
24.5\end{array}$ & $\begin{array}{l}\mathbf{P} \\
\text { Ry } \\
\text { Ry } \\
\mathbf{P} \\
\mathbf{P}\end{array}$ & & $\begin{array}{l}\text { I, II } \\
\text { I, II } \\
\text { I, II } \\
\text { I, II } \\
\text { I, II }\end{array}$ & $\begin{array}{lll}13 & 3 & 22.308 \\
13 & 2 & 48.931 \\
13 & 2 & 32.388 \\
13 & 2 & 15.926 \\
13 & 1 & 11.464\end{array}$ & $\begin{array}{l}-.122 \\
-.139 \\
-.132 \\
-.154 \\
-.096\end{array}$ & $\begin{array}{l}\mathrm{C} \\
\mathrm{C} \\
\mathrm{C} \\
\mathrm{C} \\
\mathrm{C}\end{array}$ & $\begin{array}{rrr}- & 346 & 2.60 \\
- & 342 & 40.36 \\
- & 341 & 1.96 \\
-3 & 39 & 24.19 \\
- & 333 & 0.94\end{array}$ & $\begin{array}{l}-.20 \\
+.74 \\
-.06 \\
-.59 \\
+.26\end{array}$ \\
\hline $\begin{array}{rr} & 27.4 \\
& 30.4 \\
\text { May } & 1.4 \\
& 2.4\end{array}$ & $\begin{array}{l}\mathrm{P} \\
\mathrm{P} \\
\mathrm{P} \\
\mathrm{Ry}\end{array}$ & W & $\begin{array}{l}\text { I, II } \\
\text { I, II } \\
\text { I, II } \\
\text { I, II }\end{array}$ & $\begin{array}{rrr}13 & 0 & 24.623 \\
12 & 59 & 39 . \\
12 & 59 & 24.543 \\
12 & 59 & 9.980\end{array}$ & $\begin{array}{l}-.087 \\
-.155 \\
-.117 \\
-.150\end{array}$ & $\begin{array}{l}\mathrm{C} \\
\mathrm{C} \\
\mathrm{C} \\
\mathrm{C}\end{array}$ & $\begin{array}{rrr}-328 & 27.67 \\
-324 & 5.20 \\
-322 & 40.94 \\
- & 321 & 18.51\end{array}$ & $\begin{array}{r}-.57 \\
+.30 \\
+\quad .26 \\
-.11\end{array}$ \\
\hline
\end{tabular}


NINE-INCH TRANSIT CIRCLE OBSERVATIONS, 1913-1926

\begin{tabular}{|c|c|c|c|c|c|c|c|c|}
\hline \multicolumn{9}{|c|}{$\mathbf{S A T} \mathbf{T} \mathbf{R}$} \\
\hline Date & Obsr. & $\mathrm{Cl}$. & Limb & $\underset{\text { of Center }}{\text { App. R. A. }}$ & $\begin{array}{l}\text { Corr. to } \\
\text { Am. Eph. }\end{array}$ & Limb & $\begin{array}{l}\text { App. Decl. } \\
\text { of Center }\end{array}$ & $\begin{array}{l}\text { Corr. to } \\
\text { Am. Eph. }\end{array}$ \\
\hline \begin{tabular}{cc}
\multicolumn{2}{c}{1924} \\
Mar. & 19.6 \\
& 29.6 \\
Apr. & 12.5 \\
& 14.5 \\
& 16.5
\end{tabular} & $\begin{array}{l}\mathrm{Bn} \\
\mathrm{Bn} \\
\mathrm{P} \\
\mathrm{Ry} \\
\mathrm{Ry}\end{array}$ & $\begin{array}{l}\mathrm{W} \\
\mathrm{W} \\
\mathrm{E}\end{array}$ & $\begin{array}{l}\text { I, II } \\
\text { I, II } \\
\text { I, II } \\
\text { I, II } \\
\text { I, II }\end{array}$ & $\begin{array}{rrr}\mathrm{h} & \mathrm{m} & \mathrm{s} \\
13 & 59 & 49.525 \\
13 & 57 & 26.528 \\
13 & 53 & 37.248 \\
13 & 53 & 2.964 \\
13 & 52 & 28.507\end{array}$ & $\begin{array}{l}\mathrm{s} \\
-0.085 \\
-\quad .112 \\
-.102 \\
-.086 \\
-.073\end{array}$ & $\begin{array}{l}\mathrm{C} \\
\mathrm{C} \\
\mathrm{C} \\
\mathrm{C} \\
\mathrm{C}\end{array}$ & 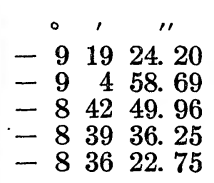 & $\begin{array}{r}\prime \prime \\
+0.30 \\
+\quad .59 \\
+.44 \\
+.55 \\
+\quad .55\end{array}$ \\
\hline $\begin{array}{rr} & 19.5 \\
& 21.5 \\
& 29.5 \\
\text { May } \quad 1.5 \\
\quad 5.5\end{array}$ & $\begin{array}{l}\mathrm{M} \\
\mathrm{Bn} \\
\mathrm{M} \\
\mathrm{Bn} \\
\mathrm{M}\end{array}$ & & $\begin{array}{l}\text { I, II } \\
\text { I, II } \\
\text { I, II } \\
\text { I, II } \\
\text { I, II }\end{array}$ & $\begin{array}{rrr}13 & 51 & 36.572 \\
13 & 51 & 2.052 \\
13 & 48 & 45.171 \\
13 & 48 & 11.623 \\
13 & 47 & 6.040\end{array}$ & $\begin{array}{l}-.158 \\
=.088 \\
=.109 \\
-.167 \\
-.120\end{array}$ & $\begin{array}{l}\mathrm{C} \\
\mathrm{C} \\
\mathrm{C} \\
\mathrm{C} \\
\mathrm{C}\end{array}$ & $\begin{array}{rrr}-8 & 31 & 33.95 \\
-8 & 28 & 22.98 \\
-8 & 15 & 58.20 \\
=8 & 12 & 58.67 \\
-8 & 7 & 10.86\end{array}$ & $\begin{array}{l}+.55 \\
+.42 \\
+.50 \\
+.73 \\
+.94\end{array}$ \\
\hline \begin{tabular}{r|}
6.5 \\
13.4 \\
16.4 \\
22.4 \\
30.4
\end{tabular} & $\begin{array}{l}P \\
M \\
P \\
R y \\
B n\end{array}$ & $\mathrm{E}$ & $\begin{array}{l}\text { I, II } \\
\text { I, II } \\
\text { I, II } \\
\text { I, II } \\
\text { I, II }\end{array}$ & $\begin{array}{rrr}13 & 46 & 49.946 \\
13 & 45 & 2.072 \\
13 & 44 & 18.790 \\
13 & 42 & 58.380 \\
13 & 41 & 25.709\end{array}$ & $\begin{array}{l}-.134 \\
=.158 \\
-.130 \\
-.080 \\
-.131\end{array}$ & $\begin{array}{l}\mathrm{C} \\
\mathrm{C} \\
\mathrm{C} \\
\mathrm{C} \\
\mathrm{C}\end{array}$ & $\begin{array}{rrr}-8 & 5 & 46.48 \\
-7 & 56 & 30.69 \\
-7 & 52 & 52.03 \\
-7 & 46 & 17.74 \\
-7 & 39 & 7.25\end{array}$ & $\begin{array}{l}+.92 \\
+.51 \\
+1.07 \\
+.76 \\
+1.15\end{array}$ \\
\hline
\end{tabular}




\begin{tabular}{|c|c|c|c|c|c|c|c|c|}
\hline \multicolumn{9}{|c|}{$\mathbf{U} \mathbf{R} \mathbf{N} \mathbf{U} \mathbf{S}$} \\
\hline Date & Obsr. & Cl. & Limb & $\begin{array}{l}\text { App. R. A. } \\
\text { of Center }\end{array}$ & $\begin{array}{l}\text { Corr. to } \\
\text { Am. Eph. }\end{array}$ & Limb & $\begin{array}{l}\text { App. Decl. } \\
\text { of Center }\end{array}$ & $\begin{array}{l}\text { Corr. to } \\
\text { Am. Eph. }\end{array}$ \\
\hline \begin{tabular}{rr}
\multicolumn{2}{c}{1914} \\
July $\quad 7.6$ \\
8.6 \\
9.6 \\
19.5 \\
20.5
\end{tabular} & $\begin{array}{l}\mathrm{P} \\
\mathrm{M} \\
\mathrm{P} \\
\mathrm{S} \\
\mathrm{M}\end{array}$ & $\mathbf{E}$ & $\begin{array}{l}\mathrm{C} \\
\mathrm{C} \\
\mathrm{C} \\
\mathrm{C} \\
\mathrm{C}\end{array}$ & \begin{tabular}{rrr}
$\mathrm{h}$ & $\mathrm{m}$ & \multicolumn{1}{c}{$\mathrm{s}$} \\
20 & 53 & 2.939 \\
20 & 52 & 54.481 \\
20 & 52 & 45.992 \\
20 & 51 & 16.238 \\
20 & 51 & 6.910
\end{tabular} & $\begin{array}{l}\text { s } \\
+0.199 \\
+.171 \\
+.202 \\
+.158 \\
+.170\end{array}$ & $\begin{array}{l}\mathrm{C} \\
\mathrm{C} \\
\mathrm{C} \\
\mathrm{C} \\
\mathrm{C}\end{array}$ & \begin{tabular}{rcr}
$\circ$ & $\prime$ & \multicolumn{1}{c}{} \\
-18 & 13 & 43.51 \\
-18 & 14 & 18.91 \\
-18 & 14 & 54.80 \\
-18 & 21 & 4.67 \\
-18 & 21 & 43.11
\end{tabular} & $\begin{array}{r} \\
\quad \prime \prime \\
+0.79 \\
+\quad .59 \\
+.30 \\
+.93 \\
+\quad .69\end{array}$ \\
\hline $\begin{array}{rr} & 21.5 \\
22.5 \\
\text { Aug. } \\
17.5 \\
18.5 \\
19.5\end{array}$ & $\begin{array}{l}\mathrm{P} \\
\mathrm{P} \\
\mathrm{Sr} \\
\mathrm{M} \\
\mathrm{S}\end{array}$ & & $\begin{array}{l}\mathrm{C} \\
\mathrm{C} \\
\mathrm{C} \\
\mathrm{C} \\
\mathrm{C}\end{array}$ & $\begin{array}{lll}20 & 50 & 57.515 \\
20 & 50 & 48.175 \\
20 & 46 & 38.864 \\
20 & 46 & 29.729 \\
20 & 46 & 20.496\end{array}$ & $\begin{array}{l}+.165 \\
+.265 \\
+.094 \\
+.189 \\
+.126\end{array}$ & $\begin{array}{l}\mathrm{C} \\
\mathrm{C} \\
\mathrm{C} \\
\mathrm{C} \\
\mathrm{C}\end{array}$ & $\begin{array}{rrr}-18 & 22 & 21.24 \\
-18 & 22 & 59.69 \\
-18 & 39 & 28.85 \\
-18 & 40 & 4.62 \\
-18 & 40 & 39.70\end{array}$ & $\begin{array}{l}+.96 \\
+1.01 \\
+.55 \\
+.48 \\
+.80\end{array}$ \\
\hline $\begin{array}{rr} & 20.5 \\
22.4 \\
30.4 \\
31.4 \\
\text { Sept. } 1.4\end{array}$ & $\begin{array}{l}\mathrm{Sr} \\
\mathrm{S} \\
\mathrm{S} \\
\mathrm{Sr} \\
\mathrm{M}\end{array}$ & & $\begin{array}{l}\mathrm{C} \\
\mathrm{C} \\
\mathrm{C} \\
\mathrm{C} \\
\mathrm{C}\end{array}$ & $\begin{array}{lll}20 & 46 & 11.373 \\
20 & 45 & 53.432 \\
20 & 44 & 44.787 \\
20 & 44 & 36.700 \\
20 & 44 & 28.649\end{array}$ & $\begin{array}{l}+.113 \\
+.172 \\
+.107 \\
+.150 \\
+.119\end{array}$ & $\begin{array}{l}\mathrm{C} \\
\mathrm{C} \\
\mathrm{C} \\
\mathrm{C} \\
\mathrm{C}\end{array}$ & $\begin{array}{lll}-18 & 41 & 15.29 \\
-18 & 42 & 24.06 \\
-18 & 46 & 44.00 \\
-18 & 47 & 15.37 \\
-18 & 47 & 45.68\end{array}$ & $\begin{array}{l}+.31 \\
+.64 \\
+1.10 \\
+.33 \\
+.12\end{array}$ \\
\hline $1915^{2.4}$ & $\mathrm{~S}$ & $\mathbf{E}$ & $\mathrm{C}$ & $\begin{array}{llll}20 & 44 & 20.806\end{array}$ & +.176 & $\mathrm{C}$ & $-1848 \quad 15.58$ & -.18 \\
\hline $\begin{array}{r}\text { July } 2.6 \\
13.6 \\
14.6\end{array}$ & $\begin{array}{l}\mathrm{P} \\
\mathrm{M} \\
\mathrm{Sr}\end{array}$ & W & $\begin{array}{l}\mathrm{C} \\
\mathrm{C} \\
\mathrm{C}\end{array}$ & $\begin{array}{rrr}21 & 10 & 37.358 \\
21 & 9 & 10.921 \\
21 & 9 & 2.484\end{array}$ & $\begin{array}{l}+.208 \\
+.151 \\
+.184\end{array}$ & $\begin{array}{l}\mathrm{C} \\
\mathrm{C} \\
\mathrm{C}\end{array}$ & $\begin{array}{rrr}-17 & 1 & 4.58 \\
-17 & 7 & 40.63 \\
-17 & 8 & 19.31\end{array}$ & $\begin{array}{l}+.82 \\
+.37 \\
-.01\end{array}$ \\
\hline $\begin{array}{r}17.6 \\
20.6 \\
24.5 \\
\text { Sept. } 7.5 \\
8.5\end{array}$ & $\begin{array}{l}\mathrm{S} \\
\mathrm{Sr} \\
\mathrm{M} \\
\mathrm{M} \\
\mathrm{S}\end{array}$ & & $\begin{array}{l}\mathrm{C} \\
\mathrm{C} \\
\mathrm{C} \\
\mathrm{C} \\
\mathrm{C}\end{array}$ & $\begin{array}{rrr}21 & 8 & 36.485 \\
21 & 8 & 9.858 \\
21 & 7 & 33.519 \\
21 & 0 & 44.210 \\
21 & 0 & 36.543\end{array}$ & $\begin{array}{l}+.105 \\
+.098 \\
+.149 \\
+.130 \\
+.093\end{array}$ & $\begin{array}{l}\mathrm{C} \\
\mathrm{C} \\
\mathrm{C} \\
\mathrm{C} \\
\mathrm{C}\end{array}$ & $\begin{array}{rrr}-17 & 10 & 16.34 \\
-17 & 12 & 15.69 \\
-17 & 14 & 57.50 \\
-17 & 44 & 3.55 \\
-17 & 44 & 34.58\end{array}$ & $\begin{array}{l}-.14 \\
-.19 \\
+.10 \\
+.35 \\
+.42\end{array}$ \\
\hline $\begin{array}{r}9.5 \\
10.5 \\
13.5 \\
14.5 \\
15.5\end{array}$ & $\begin{array}{l}\mathrm{M} \\
\mathrm{S} \\
\mathrm{P} \\
\mathrm{Sr} \\
\mathrm{P}\end{array}$ & & $\begin{array}{l}\mathrm{C} \\
\mathrm{C} \\
\mathrm{C} \\
\mathrm{C} \\
\mathrm{C}\end{array}$ & $\begin{array}{rrr}21 & 0 & 29.077 \\
21 & 0 & 21.666 \\
21 & 0 & 0.302 \\
20 & 59 & 53.428 \\
20 & 59 & 46.675\end{array}$ & $\begin{array}{l}+.137 \\
+.116 \\
+.142 \\
+.128 \\
+.095\end{array}$ & $\begin{array}{l}\mathrm{C} \\
\mathrm{C} \\
\mathrm{C} \\
\mathrm{C} \\
\mathrm{C}\end{array}$ & $\begin{array}{rrr}-17 & 45 & 5.56 \\
-17 & 45 & 36.32 \\
-17 & 47 & 2.19 \\
-17 & 47 & 29.59 \\
-17 & 47 & 56.05\end{array}$ & $\begin{array}{l}+.04 \\
\pm .82 \\
=.29 \\
+.19 \\
+.25\end{array}$ \\
\hline $\begin{array}{r}16.5 \\
20.5 \\
1916\end{array}$ & $\begin{array}{l}\mathrm{S} \\
\mathrm{M}\end{array}$ & W & $\stackrel{\mathrm{C}}{\mathrm{C}}$ & 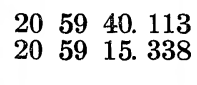 & $\begin{array}{l}+.113 \\
+.168\end{array}$ & $\stackrel{\mathrm{C}}{\mathrm{C}}$ & $\begin{array}{rrr}-17 & 48 & 22.82 \\
-17 & 50 & 0.48\end{array}$ & $\begin{array}{l}\overline{+} .22 \\
+.52\end{array}$ \\
\hline $\begin{array}{ll}\text { July } & 11.6 \\
& 12.6\end{array}$ & $\begin{array}{l}\mathrm{Sr} \\
\mathrm{M}\end{array}$ & $\mathrm{E}$ & $\begin{array}{l}\mathrm{C} \\
\mathrm{C}\end{array}$ & $\begin{array}{llll}21 & 25 & 59 . & 237 \\
21 & 25 & 51 . & 408\end{array}$ & $\begin{array}{l}+.157 \\
+.148\end{array}$ & $\begin{array}{l}\mathrm{C} \\
\mathrm{C}\end{array}$ & $\begin{array}{lll}-15 & 52 & 36.79 \\
-15 & 53 & 15.13\end{array}$ & $\begin{array}{l}+.51 \\
+.57\end{array}$ \\
\hline $\begin{array}{rr} & 23.6 \\
& 29.5 \\
\text { Aug. } & 2.5 \\
& 4.5 \\
\text { Sept. } & 2.4\end{array}$ & $\begin{array}{l}\mathrm{M} \\
\mathrm{Sr} \\
\mathrm{S} \\
\mathrm{S} \\
\mathrm{S}\end{array}$ & $\stackrel{\mathrm{E}}{\mathrm{W}}$ & $\begin{array}{l}\mathrm{C} \\
\mathrm{C} \\
\mathrm{C} \\
\mathrm{C} \\
\mathrm{C}\end{array}$ & $\begin{array}{rrr}21 & 24 & 19.180 \\
21 & 23 & 25.136 \\
21 & 22 & 47.992 \\
21 & 22 & 29.264 \\
21 & 18 & 2.801\end{array}$ & $\begin{array}{l}+.180 \\
+.246 \\
+.152 \\
+.144 \\
+.071\end{array}$ & $\begin{array}{l}\mathrm{C} \\
\mathrm{C} \\
\mathrm{C}\end{array}$ & $\begin{array}{rrr}-16 & 0 & 42.99 \\
-16 & 5 & 1.34 \\
-16 & 7 & 56.52 \\
-16 & 9 & \\
-16 & 29 & 50.18\end{array}$ & $\begin{array}{l}+.41 \\
+.76 \\
+1.18 \\
+.52\end{array}$ \\
\hline $\begin{array}{r}3.4 \\
7.4 \\
9.4 \\
11.4 \\
13.4\end{array}$ & $\begin{array}{l}\mathrm{M} \\
\mathrm{S} \\
\mathrm{M} \\
\mathrm{S} \\
\mathrm{M}\end{array}$ & & $\begin{array}{l}\mathrm{C} \\
\mathrm{C} \\
\mathrm{C} \\
\mathrm{C} \\
\mathrm{C}\end{array}$ & $\begin{array}{rrr}21 & 17 & 54.443 \\
21 & 17 & 21.829 \\
21 & 17 & 6.063 \\
21 & 16 & 50.800 \\
21 & 16 & 35.948\end{array}$ & $\begin{array}{l}+.113 \\
+.169 \\
+.113 \\
+.130 \\
+.098\end{array}$ & $\begin{array}{l}\mathrm{C} \\
\mathrm{C} \\
\mathrm{C} \\
\mathrm{C} \\
\mathrm{C}\end{array}$ & $\begin{array}{rrr}-16 & 30 & 27.58 \\
-16 & 32 & 53.48 \\
-16 & 34 & 3.31 \\
-16 & 35 & 9.74 \\
-16 & 36 & 15.50\end{array}$ & $\begin{array}{l}+.72 \\
+.32 \\
+.11 \\
+.86 \\
+.20\end{array}$ \\
\hline $\begin{array}{l}16.4 \\
19.4 \\
20.4 \\
21.4\end{array}$ & $\begin{array}{l}\mathrm{M} \\
\mathrm{P} \\
\mathrm{M} \\
\mathrm{S}\end{array}$ & & $\begin{array}{l}\mathrm{C} \\
\mathrm{C} \\
\mathrm{C} \\
\mathrm{C}\end{array}$ & $\begin{array}{llll}21 & 16 & 14.753 \\
21 & 15 & 54.616 \\
21 & 15 & 48 . & 154 \\
21 & 15 & 41.881\end{array}$ & $\begin{array}{l}+.183 \\
+.146 \\
+.104 \\
+.111\end{array}$ & $\begin{array}{l}\mathrm{C} \\
\mathrm{C} \\
\mathrm{C} \\
\mathrm{C}\end{array}$ & $\begin{array}{rrr}-16 & 37 & 48.16 \\
-16 & 39 & 15.28 \\
-16 & 39 & 42.74 \\
-16 & 40 & 9.94\end{array}$ & $\begin{array}{l}+.44 \\
+.42 \\
+.66 \\
+.36\end{array}$ \\
\hline \begin{tabular}{lr}
\multicolumn{2}{c}{1919} \\
July & 29.6 \\
Aug. & 8.6 \\
& 9.6 \\
& 11.5 \\
& 15.5
\end{tabular} & $\begin{array}{l}\mathrm{M} \\
\mathrm{M} \\
\mathrm{Sr} \\
\mathrm{Sr} \\
\mathrm{P}\end{array}$ & W & $\begin{array}{l}\mathrm{C} \\
\mathrm{C} \\
\mathrm{C} \\
\mathrm{C} \\
\mathrm{C}\end{array}$ & $\begin{array}{rrrr}22 & 12 & 2 . & 180 \\
22 & 10 & 38 . & 357 \\
22 & 10 & 29 . & 639 \\
22 & 10 & 12 . & 034 \\
22 & 9 & 36 . & 155\end{array}$ & $\begin{array}{l}+.140 \\
+.107 \\
+.119 \\
+.164 \\
+.065\end{array}$ & $\begin{array}{l}\mathrm{C} \\
\mathrm{C} \\
\mathrm{C} \\
\mathrm{C} \\
\mathrm{C}\end{array}$ & $\begin{array}{rrr}-11 & 58 & 35.05 \\
-12 & 6 & 30.20 \\
-12 & 7 & 19.70 \\
-12 & 8 & 58.75 \\
-12 & 12 & 19.15\end{array}$ & $\begin{array}{l}+.45 \\
+.70 \\
+.30 \\
+.35 \\
+.25\end{array}$ \\
\hline
\end{tabular}


NINE-INCH TRANSIT CIRCLE OBSERVATIONS, 1913-1926

\begin{tabular}{|c|c|c|c|c|c|c|c|c|}
\hline \multicolumn{9}{|c|}{ URA N U S } \\
\hline Date & Obsr. & Cl. & $\operatorname{Limb}$ & $\begin{array}{l}\text { App. R. A. } \\
\text { of Center }\end{array}$ & $\begin{array}{l}\text { Corr. to } \\
\text { Am. Eph. }\end{array}$ & $\operatorname{Limb}$ & $\begin{array}{l}\text { App. Decl. } \\
\text { of Center }\end{array}$ & $\begin{array}{l}\text { Corr. to } \\
\text { Am. Eph. }\end{array}$ \\
\hline $\begin{aligned} & 1919 \\
& \text { Aug. } 19.5 \\
& 22.5 \\
& 25.5 \\
& 26.5 \\
& 28.5\end{aligned}$ & $\begin{array}{l}\mathrm{P} \\
\mathrm{M} \\
\mathrm{M} \\
\mathrm{P} \\
\mathrm{M}\end{array}$ & $\mathrm{W}$ & $\begin{array}{l}\mathrm{C} \\
\mathrm{C} \\
\mathrm{C} \\
\mathrm{C} \\
\mathrm{C}\end{array}$ & $\begin{array}{ccc}\mathrm{h} & \mathrm{m} & \mathrm{s} \\
22 & 8 & 59.933 \\
22 & 8 & 32.531 \\
22 & 8 & 4.984 \\
22 & 7 & 55.944 \\
22 & 7 & 37.631\end{array}$ & $\begin{array}{l}\mathrm{s} \\
+0.123 \\
+.131 \\
+.054 \\
+.154 \\
+.091\end{array}$ & $\begin{array}{l}\mathrm{C} \\
\mathrm{C} \\
\mathrm{C} \\
\mathrm{C} \\
\mathrm{C}\end{array}$ & $\begin{array}{rcc}\circ & \prime & \prime \prime \\
-12 & 15 & 41.17 \\
-12 & 18 & 12.36 \\
-12 & 20 & 43.90 \\
-12 & 21 & 34.71 \\
-12 & 23 & 14.93\end{array}$ & $\begin{array}{l}\quad l \\
+0.13 \\
+.74 \\
+.60 \\
+.09 \\
-.03\end{array}$ \\
\hline $\begin{array}{r}8.5 \\
\text { Sept. } \\
12.4 \\
16.4 \\
18.4 \\
25.4\end{array}$ & $\begin{array}{l}\mathrm{Sr} \\
\mathrm{Sr} \\
\mathrm{Sr} \\
\mathrm{M} \\
\mathrm{P}\end{array}$ & & $\begin{array}{l}\mathrm{C} \\
\mathrm{C} \\
\mathrm{C}\end{array}$ & $\begin{array}{lll}22 & 5 & 59.369 \\
22 & 5 & 25.222 \\
22 & 4 & 52.175 \\
22 & 4 & \\
22 & 3 & 43.063\end{array}$ & $\begin{array}{l}+.109 \\
+.182 \\
+.205 \\
+.093\end{array}$ & $\begin{array}{l}\mathrm{C} \\
\mathrm{C} \\
\mathrm{C} \\
\mathrm{C} \\
\mathrm{C}\end{array}$ & $\begin{array}{rrr}-12 & 32 & 8.93 \\
-12 & 35 & 12.62 \\
-12 & 38 & 8.91 \\
-12 & 39 & 34.02 \\
-12 & 44 & 14.03\end{array}$ & $\begin{array}{l}\bar{T} .03 \\
+.38 \\
+.79 \\
+.78 \\
+.07\end{array}$ \\
\hline $\begin{array}{c}26.4 \\
1920\end{array}$ & $\mathbf{P}$ & $\mathrm{W}$ & $\mathrm{C}$ & $22 \quad 3 \quad 35.916$ & +.076 & $\mathrm{C}$ & $-1244 \quad 50.43$ & +.97 \\
\hline 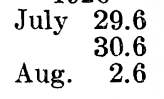 & $\begin{array}{l}\mathrm{M} \\
\mathrm{Bn} \\
\mathrm{P}\end{array}$ & $\mathrm{E}$ & $\begin{array}{l}\mathrm{C} \\
\mathrm{C}\end{array}$ & $\begin{array}{rrr}22 & 27 & 35.980 \\
22 & 27 & 28.309 \\
22 & 27 & 4.847\end{array}$ & $\begin{array}{l}+.160 \\
+: 129 \\
+.157\end{array}$ & $\begin{array}{l}\mathrm{C} \\
\mathrm{C} \\
\mathrm{C}\end{array}$ & $\begin{array}{llll}-10 & 30 & 45.07 \\
-10 & 31 & 30.34 \\
-10 & 33 & 51.14\end{array}$ & $\begin{array}{l}+.83 \\
+1.16 \\
+.26\end{array}$ \\
\hline $\begin{array}{r}3.6 \\
11.5 \\
26.5 \\
30.5 \\
\text { Sept. } \quad 2.5\end{array}$ & $\begin{array}{l}\mathrm{P} \\
\mathrm{Ry} \\
\mathrm{Ry} \\
\mathrm{Ry} \\
\mathrm{Bn}\end{array}$ & $\stackrel{\mathrm{E}}{\mathrm{W}}$ & $\begin{array}{l}\mathrm{C} \\
\mathrm{C} \\
\mathrm{C} \\
\mathrm{C} \\
\mathrm{C}\end{array}$ & $\begin{array}{lll}22 & 26 & 56.828 \\
22 & 25 & 50.012 \\
22 & 23 & 36.336 \\
22 & 23 & 0.126 \\
22 & 22 & 33.032\end{array}$ & $\begin{array}{l}+.148 \\
+: 122 \\
+: 106 \\
+.126 \\
+.082\end{array}$ & $\begin{array}{l}\mathrm{C} . \\
\mathrm{C} \\
\mathrm{C} \\
\mathrm{C} \\
\mathrm{C}\end{array}$ & $\begin{array}{rrr}-10 & 34 & 38.20 \\
-10 & 41 & 12.53 \\
-10 & 54 & 8.76 \\
-10 & 57 & 36.73 \\
-11 & 0 & 10.93\end{array}$ & $\begin{array}{l}+.80 \\
+.67 \\
+.64 \\
+.57 \\
+.87\end{array}$ \\
\hline $\begin{array}{r}3.5 \\
4.5 \\
10.5 \\
13.5 \\
16.4\end{array}$ & $\begin{array}{l}\mathrm{Bn} \\
\mathrm{Ry} \\
\mathrm{Bn} \\
\mathrm{P} \\
\mathrm{Ry}\end{array}$ & & $\begin{array}{l}\mathrm{C} \\
\mathrm{C} \\
\mathrm{C} \\
\mathrm{C} \\
\mathrm{C}\end{array}$ & $\begin{array}{llll}22 & 22 & 24.123 \\
22 & 22 & 15.161 \\
22 & 21 & 22.221 \\
22 & 20 & 56.425 \\
22 & 20 & 31.154\end{array}$ & $\begin{array}{l}+.153 \\
+.141 \\
+.141 \\
+.145 \\
+.084\end{array}$ & $\begin{array}{l}\mathrm{C} \\
\mathrm{C} \\
\mathrm{C} \\
\mathrm{C} \\
\mathrm{C}\end{array}$ & $\begin{array}{rrr}-11 & 1 & 1.76 \\
-11 & 1 & 53.50 \\
-11 & 6 & 52.26 \\
-11 & 9 & 17.53 \\
-11 & 11 & 39.10\end{array}$ & $\begin{array}{l}+1.14 \\
+.30 \\
+1.14 \\
+.87 \\
+.50\end{array}$ \\
\hline $\begin{array}{r}17.4 \\
18.4 \\
24.4 \\
1921\end{array}$ & $\begin{array}{l}\text { Ry } \\
P \\
\text { Ry }\end{array}$ & & $\begin{array}{l}\mathrm{C} \\
\mathrm{C} \\
\mathrm{C}\end{array}$ & $\begin{array}{lll}22 & 20 & 22.837 \\
22 & 20 & 14.739 \\
22 & 19 & 27.511\end{array}$ & $\begin{array}{l}+.027 \\
+.109 \\
+.091\end{array}$ & $\begin{array}{l}\mathrm{C} \\
\mathrm{C} \\
\mathrm{C}\end{array}$ & $\begin{array}{lll}-11 & 12 & 25.08 \\
-11 & 13 & 11.14 \\
-11 & 17 & 32.49\end{array}$ & $\begin{array}{l}+.62 \\
+.16 \\
+.51\end{array}$ \\
\hline Aug. $\quad 4.6$ & $\mathrm{Ry}$ & & C & $22 \quad 42 \quad 20.529$ & +.109 & $\mathrm{C}$ & $\begin{array}{lll}-9 & 4 & 16.87\end{array}$ & +.63 \\
\hline $\begin{array}{r}9.6 \\
10.6 \\
18.5 \\
19.5 \\
21.5\end{array}$ & $\begin{array}{l}\mathrm{P} \\
\mathrm{P} \\
\mathrm{Ry} \\
\mathrm{Ry} \\
\mathrm{Ry}\end{array}$ & & $\begin{array}{l}\mathrm{C} \\
\mathrm{C} \\
\mathrm{C} \\
\mathrm{C} \\
\mathrm{C}\end{array}$ & $\begin{array}{lll}22 & 41 & 41.005 \\
22 & 41 & 32.831 \\
22 & 40 & 25.099 \\
22 & 40 & 16.447 \\
22 & 39 & 58.871\end{array}$ & $\begin{array}{l}+.175 \\
+.161 \\
+.069 \\
+.127 \\
+.091\end{array}$ & $\begin{array}{l}\mathrm{C} \\
\mathbf{C} \\
\mathbf{C} \\
\mathrm{C} \\
\mathrm{C}\end{array}$ & 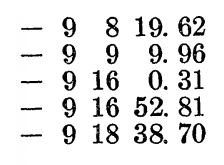 & $\begin{array}{r}+.98 \\
+.54 \\
+1.39 \\
+1.49 \\
+1.30\end{array}$ \\
\hline $\begin{array}{r}22.5 \\
27.5 \\
\text { Sept. } 7.5 \\
13.5 \\
14.5\end{array}$ & $\begin{array}{l}\text { Ry } \\
\text { Ry } \\
\text { Bn } \\
\mathrm{P} \\
\mathrm{Bn}\end{array}$ & & $\begin{array}{l}\mathrm{C} \\
\mathrm{C} \\
\mathrm{C} \\
\mathrm{C} \\
\mathrm{C}\end{array}$ & $\begin{array}{lll}22 & 39 & 50.045 \\
22 & 39 & 5.526 \\
22 & 37 & 26.820 \\
22 & 36 & 34.063 \\
22 & 36 & 25.379\end{array}$ & $\begin{array}{l}+.085 \\
+.126 \\
+.140 \\
+.173 \\
+.139\end{array}$ & $\begin{array}{l}\mathrm{C} \\
\mathrm{C} \\
\mathrm{C} \\
\mathrm{C} \\
\mathrm{C}\end{array}$ & 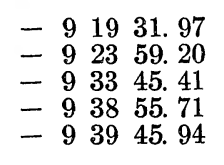 & $\begin{array}{l}+1.13 \\
+1.00 \\
+.79 \\
+.79 \\
+1.16\end{array}$ \\
\hline $\begin{array}{rr} & 19.4 \\
& 22.4 \\
30.4 \\
\text { Oct. } \quad 1.4\end{array}$ & $\begin{array}{l}\mathrm{P} \\
\mathrm{Ry} \\
\mathrm{M}\end{array}$ & & $\begin{array}{l}\mathrm{C} \\
\mathrm{C} \\
\mathrm{C} \\
\mathrm{C}\end{array}$ & $\begin{array}{lll}22 & 35 & 42.946 \\
22 & 35 & 18.295 \\
22 & 34 & 16.283 \\
22 & 34 & 9.095\end{array}$ & $\begin{array}{l}+.096 \\
+.075 \\
+.013 \\
+.125\end{array}$ & $\begin{array}{l}\mathrm{C} \\
\mathrm{C} \\
\mathrm{C} \\
\mathrm{C}\end{array}$ & 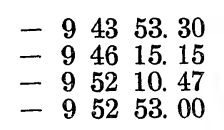 & $\begin{array}{l}+.70 \\
+1.55 \\
+1.93 \\
+1.00\end{array}$ \\
\hline \begin{tabular}{cc}
\multicolumn{2}{c}{1922} \\
Aug. $\quad 9.6$ \\
15.6 \\
18.5 \\
21.5 \\
22.5
\end{tabular} & $\begin{array}{l}\mathrm{M} \\
\mathrm{P} \\
\mathrm{Ry} \\
\mathrm{Ry} \\
\mathrm{Ry}\end{array}$ & $\begin{array}{l}\mathrm{W} \\
\mathrm{E}_{1}\end{array}$ & $\begin{array}{l}\mathrm{C} \\
\mathbf{C} \\
\mathbf{C} \\
\mathbf{C} \\
\mathbf{C}\end{array}$ & $\begin{array}{lll}22 & 57 & 4.218 \\
22 & 56 & 16 . \\
22 & 554 \\
22 & 55.731 \\
22 & 55 & 26.293 \\
22 & 55 & 17.702\end{array}$ & $\begin{array}{l}+.078 \\
+.114 \\
+.161 \\
+.163 \\
+.162\end{array}$ & $\begin{array}{l}\mathrm{C} \\
\mathrm{C} \\
\mathrm{C} \\
\mathrm{C} \\
\mathrm{C}\end{array}$ & $\begin{array}{rrr}-7 & 34 & 55.95 \\
= & 39 & 57.57 \\
=7 & 42 & 31.47 \\
=7 & 45 & 10.30 \\
-7 & 46 & 4.54\end{array}$ & $\begin{array}{l}+1.15 \\
+.17 \\
+1.63 \\
+1.50 \\
+.66\end{array}$ \\
\hline $\begin{array}{r}23.5 \\
28.5 \\
\text { Sept. } 5.5 \\
13.5 \\
14.5\end{array}$ & $\begin{array}{l}\text { Bn } \\
\text { Ry } \\
\text { My } \\
\text { Ry } \\
\text { Ry }\end{array}$ & $\mathbf{E}$ & $\begin{array}{l}\mathbf{C} \\
\mathbf{C} \\
\mathbf{C} \\
\mathbf{C} \\
\mathbf{C}\end{array}$ & $\begin{array}{rrr}22 & 55 & 9.078 \\
22 & 54 & 25.285 \\
22 & 53 & 14.007 \\
22 & 52 & 2.999 \\
22 & 51 & 54.228\end{array}$ & $\begin{array}{l}+.178 \\
+: 165 \\
+.087 \\
+.099 \\
+.108\end{array}$ & $\begin{array}{l}\mathrm{C} \\
\mathrm{C} \\
\mathrm{C} \\
\mathrm{C} \\
\mathrm{C}\end{array}$ & 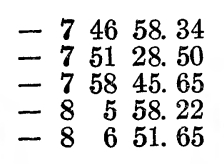 & $\begin{array}{l}+.46 \\
+1.60 \\
+1.85 \\
+1.68 \\
+1.45\end{array}$ \\
\hline
\end{tabular}




\begin{tabular}{|c|c|c|c|c|c|c|c|c|}
\hline \multicolumn{9}{|c|}{$\mathbf{U} \mathbf{R} \mathbf{N} \mathbf{U} \mathbf{S}$} \\
\hline Date & Obsr. & Cl. & Limb & $\begin{array}{l}\text { App. R. A. } \\
\text { of Center }\end{array}$ & $\begin{array}{l}\text { Corr. to } \\
\text { Am. Eph. }\end{array}$ & Limb & $\begin{array}{l}\text { App. Decl. } \\
\text { of Center }\end{array}$ & $\begin{array}{l}\text { Corr. to } \\
\text { Am. Eph. }\end{array}$ \\
\hline $\begin{aligned} & 1922 \\
& \text { Sept. } 15.5 \\
& 22.4 \\
& 26.4 \\
& 27.4 \\
& 28.4\end{aligned}$ & $\begin{array}{l}\mathrm{P} \\
\mathrm{M} \\
\mathrm{M} \\
\mathrm{Ry} \\
\mathrm{Ry}\end{array}$ & $\mathbf{E}$ & $\begin{array}{l}\mathrm{C} \\
\mathrm{C} \\
\mathrm{C} \\
\mathrm{C}\end{array}$ & $\begin{array}{rrr}\mathrm{h} & \mathrm{m} & \mathrm{s} \\
22 & 52 & \\
22 & 50 & 45.576 \\
22 & 50 & 12.756 \\
22 & 50 & 4.732 \\
22 & 49 & 56.765\end{array}$ & $\begin{array}{c}\mathrm{s} \\
+0.086 \\
+.126 \\
+.122 \\
+.085\end{array}$ & $\begin{array}{l}\mathrm{C} \\
\mathrm{C} \\
\mathrm{C} \\
\mathrm{C} \\
\mathrm{C}\end{array}$ & 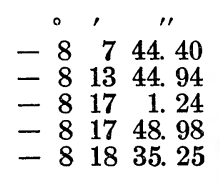 & $\begin{aligned} & \prime \prime \\
&+1.60 \\
&+1.16 \\
&+1.16 \\
&+1.22 \\
&+2.05\end{aligned}$ \\
\hline Oct. $\begin{array}{r}30.4 \\
1923\end{array}$ & $\stackrel{\mathrm{P}}{\mathrm{P}}$ & & $\begin{array}{l}\mathrm{C} \\
\mathrm{C}\end{array}$ & $\begin{array}{lll}22 & 49 & 41 . \\
22 & 49 & 25.940\end{array}$ & $\begin{array}{l}+.169 \\
+.130\end{array}$ & $\begin{array}{l}\mathrm{C} \\
\mathrm{C}\end{array}$ & $\begin{array}{rrr}-8 & 20 & 8.58 \\
-8 & 21 & 38.37\end{array}$ & $\begin{array}{l}+1.42 \\
+1.73\end{array}$ \\
\hline $\begin{array}{ll}\text { Aug. } & 13.6 \\
& 15.6\end{array}$ & $\begin{array}{l}\mathrm{M} \\
\mathrm{Bn}\end{array}$ & & $\begin{array}{l}\mathrm{C} \\
\mathrm{C}\end{array}$ & 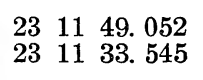 & $\begin{array}{l}+.172 \\
+.105\end{array}$ & $\begin{array}{l}\mathrm{C} \\
\mathrm{C}\end{array}$ & $\begin{array}{rrr}-6 & 258.75 \\
-6 & 438.61\end{array}$ & $\begin{array}{l}+1.55 \\
+1.29\end{array}$ \\
\hline $\begin{array}{l}22.5 \\
23.5 \\
25.5 \\
27.5 \\
30.5\end{array}$ & $\begin{array}{l}\mathrm{M} \\
\mathrm{P} \\
\mathrm{Bn} \\
\mathrm{Ry} \\
\mathrm{M}\end{array}$ & & $\begin{array}{l}\mathrm{C} \\
\mathrm{C} \\
\mathrm{C} \\
\mathrm{C} \\
\mathrm{C}\end{array}$ & $\begin{array}{rrr}23 & 10 & 37.036 \\
23 & 10 & 28.664 \\
23 & 10 & 11.854 \\
23 & 9 & 54.703 \\
23 & 9 & 28.756\end{array}$ & $\begin{array}{l}+.156 \\
+.134 \\
+.194 \\
+.133 \\
+.136\end{array}$ & $\begin{array}{l}\mathrm{C} \\
\mathrm{C} \\
\mathrm{C} \\
\mathrm{C} \\
\mathrm{C}\end{array}$ & $\begin{array}{llll}- & 6 & 10 & 41.84 \\
- & 6 & 11 & 34.87 \\
- & 6 & 13 & 21.64 \\
- & 6 & 15 & 10.68 \\
-6 & 17 & 55.52\end{array}$ & $\begin{array}{l}+.66 \\
+\quad .93 \\
+1.66 \\
+1.22 \\
+.78\end{array}$ \\
\hline $\begin{array}{r}31.5 \\
\text { Sept. } 1.5 \\
10.5 \\
11.5 \\
28.4\end{array}$ & $\begin{array}{l}\mathrm{Ry} \\
\mathrm{Bn} \\
\mathrm{Ry} \\
\mathrm{Bn} \\
\mathrm{P}\end{array}$ & & $\begin{array}{l}\mathrm{C} \\
\mathrm{C} \\
\mathrm{C} \\
\mathrm{C} \\
\mathrm{C}\end{array}$ & $\begin{array}{lll}23 & 9 & 20.027 \\
23 & 9 & 11.213 \\
23 & 7 & 51.574 \\
23 & 7 & 42.697 \\
23 & 5 & 16.199\end{array}$ & $\begin{array}{l}+.137 \\
+.083 \\
+.134 \\
+.137 \\
+.119\end{array}$ & $\begin{array}{l}\mathrm{C} \\
\mathrm{C} \\
\mathrm{C} \\
\mathrm{C} \\
\mathrm{C}\end{array}$ & $\begin{array}{llll}- & 6 & 18 & 50.08 \\
- & 6 & 19 & 45.77 \\
- & 6 & 28 & 6.04 \\
- & 6 & 29 & 1.86 \\
- & 6 & 44 & 7.96\end{array}$ & $\begin{array}{l}+1.52 \\
+1.23 \\
+1.56 \\
+1.14 \\
+1.04\end{array}$ \\
\hline $\begin{array}{r}\text { Oct. } 1.4 \\
8.4 \\
11.4 \\
1924\end{array}$ & $\begin{array}{l}\mathrm{P} \\
\mathrm{P} \\
\mathrm{Ry}\end{array}$ & & $\begin{array}{l}\mathrm{C} \\
\mathrm{C} \\
\mathrm{C}\end{array}$ & $\begin{array}{lll}23 & 4 & 51.936 \\
23 & 3 & 58.392 \\
23 & 3 & 36.931\end{array}$ & $\begin{array}{l}+.086 \\
+.112 \\
+.121\end{array}$ & $\begin{array}{l}\mathrm{C} \\
\mathrm{C} \\
\mathrm{C}\end{array}$ & $\begin{array}{rrr}-6 & 46 & 36.19 \\
-6 & 52 & 0.33 \\
-6 & 54 & 9.63\end{array}$ & $\begin{array}{l}+.81 \\
+1.47 \\
+1.37\end{array}$ \\
\hline Aug. 14.6 & $\mathrm{P}$ & & C & $23 \quad 2643.870$ & +.160 & $\mathrm{C}$ & -42754.08 & +1.32 \\
\hline $\begin{array}{l}15.6 \\
18.6 \\
19.6 \\
20.6 \\
21.6\end{array}$ & $\begin{array}{l}\text { Ry } \\
\text { Ry } \\
\text { P } \\
B n \\
P\end{array}$ & & $\begin{array}{l}\mathrm{C} \\
\mathrm{C} \\
\mathrm{C} \\
\mathrm{C} \\
\mathrm{C}\end{array}$ & $\begin{array}{rrr}23 & 26 & 36.430 \\
23 & 26 & 13.584 \\
23 & 26 & 5.816 \\
23 & 25 & 57.957 \\
23 & 25 & 49.960\end{array}$ & $\begin{array}{l}+.140 \\
+.104 \\
+.126 \\
+.137 \\
+.090\end{array}$ & $\begin{array}{l}\mathrm{C} \\
\mathrm{C} \\
\mathrm{C} \\
\mathrm{C} \\
\mathrm{C}\end{array}$ & $\begin{array}{rrr}-428 & 43.25 \\
-431 & 12.78 \\
-432 & 3.85 \\
-432 & 55.34 \\
-4 & 33 & 46.67\end{array}$ & $\begin{array}{l}+.95 \\
+1.02 \\
+.95 \\
+.86 \\
+1.33\end{array}$ \\
\hline $\begin{array}{l}26.5 \\
27.5 \\
28.5 \\
29.5 \\
30.5\end{array}$ & $\begin{array}{l}\mathrm{P} \\
\mathrm{M} \\
\mathrm{P} \\
\mathrm{Ry} \\
\mathrm{M}\end{array}$ & & $\begin{array}{l}\mathrm{C} \\
\mathrm{C} \\
\mathrm{C} \\
\mathrm{C} \\
\mathrm{C}\end{array}$ & $\begin{array}{rrr}23 & 25 & 9.201 \\
23 & 25 & 0.796 \\
23 & 24 & 52.410 \\
23 & 24 & 43.919 \\
23 & 24 & 35.345\end{array}$ & $\begin{array}{l}+.181 \\
+.146 \\
+.180 \\
+.169 \\
+.125\end{array}$ & $\begin{array}{l}\mathrm{C} \\
\mathrm{C} \\
\mathrm{C} \\
\mathrm{C} \\
\mathrm{C}\end{array}$ & $\begin{array}{rrr}-438 & 12.24 \\
-439 & 5.87 \\
-440 & 0.49 \\
-440 & 55.17 \\
-4 & 41 & 50.43\end{array}$ & $\begin{array}{l}+1.26 \\
+1.83 \\
+1.71 \\
+1.83 \\
+1.57\end{array}$ \\
\hline $\begin{array}{r}\text { Sept. } \\
4.5 \\
9.5 \\
10.5 \\
11.5\end{array}$ & $\begin{array}{l}\mathrm{P} \\
\mathrm{P} \\
\mathrm{Ry} \\
\mathrm{P}\end{array}$ & $\mathbf{E}$ & $\begin{array}{l}\mathrm{C} \\
\mathrm{C} \\
\mathrm{C} \\
\mathrm{C}\end{array}$ & $\begin{array}{rrr}23 & 23 & 52.072 \\
23 & 23 & 8.227 \\
23 & 22 & 59.336 \\
23 & 22 & 50.519\end{array}$ & $\begin{array}{l}+.102 \\
+.207 \\
+.146 \\
+.169\end{array}$ & $\begin{array}{l}\mathrm{C} \\
\mathrm{C} \\
\mathrm{C} \\
\mathrm{C}\end{array}$ & $\begin{array}{rrr}-446 & 28.71 \\
-451 & 9.69 \\
-452 & 6.01 \\
-4 & 53 & 3.01\end{array}$ & $\begin{array}{l}+1.59 \\
+2.11 \\
+2.19 \\
+1.49\end{array}$ \\
\hline
\end{tabular}


NINE-INCH TRANSIT CIRCLE OBSERVATIONS, 1913-1926

\begin{tabular}{|c|c|c|c|c|c|c|c|c|}
\hline \multicolumn{9}{|c|}{$\mathbf{N E P T U N E}$} \\
\hline Date & Obsr. & $\mathrm{Cl}$. & Limb & $\begin{array}{l}\text { App. R. A. } \\
\text { of Center }\end{array}$ & $\begin{array}{l}\text { Corr. to } \\
\text { Am. Eph. }\end{array}$ & Limb & $\begin{array}{l}\text { App. Decl. } \\
\text { of Center }\end{array}$ & $\begin{array}{l}\text { Corr. to } \\
\text { Am. Eph. }\end{array}$ \\
\hline $\begin{array}{c}1913 \\
\text { Dee. } 27.6 \\
29.6 \\
1914\end{array}$ & $\stackrel{\mathrm{M}}{\mathrm{P}}$ & $\mathrm{E}$ & $\stackrel{\mathrm{C}}{\mathrm{C}}$ & 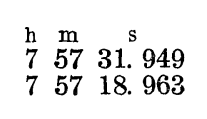 & $\begin{array}{l}\text { s } \\
-0.121 \\
-.077\end{array}$ & $\underset{\mathrm{C}}{\mathrm{C}}$ & $\begin{array}{rcc}\circ & \prime & \prime \prime \\
+20 & 15 & 4.67 \\
+20 & 15 & 41.65\end{array}$ & $\begin{array}{l}-0.13 \\
-.35\end{array}$ \\
\hline $\begin{array}{r}\text { Jan. } \quad 13.5 \\
15.5\end{array}$ & $\begin{array}{l}\mathrm{M} \\
\mathrm{M}\end{array}$ & & $\begin{array}{l}\mathrm{C} \\
\mathrm{C}\end{array}$ & $\begin{array}{lll}7 & 55 & 36.008 \\
7 & 55 & 21.816\end{array}$ & $\begin{array}{l}-.072 \\
-.134\end{array}$ & $\underset{\mathrm{C}}{\mathrm{C}}$ & $\begin{array}{r}+202035.30 \\
+2021 \\
+24.84\end{array}$ & \pm .10 \\
\hline $\begin{array}{r}17.5 \\
27.5 \\
28.5 \\
29.5 \\
\text { Feb. } \quad 7.4\end{array}$ & $\begin{array}{l}\mathrm{P} \\
\mathrm{P} \\
\mathrm{M} \\
\mathrm{Ep} \\
\mathrm{M}\end{array}$ & $\stackrel{\mathrm{E}}{\mathrm{W}}$ & $\begin{array}{l}\mathrm{C} \\
\mathrm{C} \\
\mathrm{C} \\
\mathrm{C} \\
\mathrm{C}\end{array}$ & $\begin{array}{lrr}7 & 55 & 7.632 \\
7 & 53 & 57.394 \\
7 & 53 & 50.448 \\
7 & 53 & 43.530 \\
7 & 52 & 43.548\end{array}$ & $\begin{array}{l}=.178 \\
=: 116 \\
=: 152 \\
=: 190 \\
=.142\end{array}$ & $\begin{array}{l}\mathrm{C} \\
\mathrm{C} \\
\mathrm{C} \\
\mathrm{C} \\
\mathrm{C}\end{array}$ & 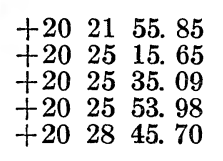 & $\begin{array}{l}+.25 \\
\pm .15 \\
\pm .11 \\
-.82 \\
.00\end{array}$ \\
\hline $\begin{array}{r}8.4 \\
9.4\end{array}$ & $\mathrm{M}$ & & $\stackrel{\mathrm{C}}{\mathrm{C}}$ & $\begin{array}{lll}7 & 52 & 37.150 \\
7 & 52 & 30.852\end{array}$ & $\begin{array}{l}-.130 \\
-.088\end{array}$ & $\underset{\mathrm{C}}{\mathrm{C}}$ & $\begin{array}{r}+2029 \\
+2029.64 \\
+21.65\end{array}$ & $\begin{array}{l}-.36 \\
-.45\end{array}$ \\
\hline 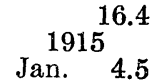 & $\mathbf{P}$ & & $\mathrm{C}$ & $\begin{array}{llll}7 & 51 & 48.310\end{array}$ & -.120 & $\mathrm{C}$ & +203124.19 & +.39 \\
\hline $\begin{array}{l}\text { Jan. } \\
\quad 4.5 \\
\\
7.5\end{array}$ & $S$ & & $\mathrm{C}$ & $\begin{array}{lll}8 & 6 & 14.098\end{array}$ & -.082 & C & $+1954 \quad 3.19$ & -.21 \\
\hline $\begin{array}{r}7.5 \\
8.5 \\
9.5 \\
13.5 \\
21.5\end{array}$ & $\begin{array}{l}\mathrm{Sr} \\
\mathrm{Sr} \\
\mathrm{M} \\
\mathrm{S} \\
\mathrm{Sr}\end{array}$ & & $\begin{array}{l}\mathrm{C} \\
\mathrm{C} \\
\mathrm{C} \\
\mathrm{C}\end{array}$ & $\begin{array}{lll}8 & 5 & 53.773 \\
8 & 5 & 46.916 \\
8 & 5 & 40.027 \\
8 & 5 & 12.392 \\
8 & 4 & \end{array}$ & $\begin{array}{l}=.137 \\
=.154 \\
=.173 \\
=.058\end{array}$ & $\begin{array}{l}\mathrm{C} \\
\mathrm{C} \\
\mathrm{C} \\
\mathrm{C} \\
\mathrm{C}\end{array}$ & $\begin{array}{rrr}+1955 & 5.36 \\
+1955 & 26.30 \\
+1955 & 48.11 \\
+1957 & 12.57 \\
+20 & 0 & 4.43\end{array}$ & $\begin{array}{l}=.34 \\
\bar{y} .40 \\
\pm .31 \\
-.33 \\
-.67\end{array}$ \\
\hline $\begin{array}{r}25.5 \\
28.5 \\
29.5 \\
\text { Feb. } 9.4 \\
\\
10.4\end{array}$ & $\begin{array}{l}\mathrm{P} \\
\mathrm{S} \\
\mathrm{M} \\
\mathrm{S} \\
\mathrm{P}\end{array}$ & $\underset{E}{W}$ & $\begin{array}{l}\mathrm{C} \\
\mathrm{C} \\
\mathrm{C} \\
\mathrm{C} \\
\mathrm{C}\end{array}$ & $\begin{array}{rrr}8 & 3 & 48.094 \\
8 & 3 & 27.195 \\
8 & 3 & 20.385 \\
8 & 2 & 6.697 \\
8 & 2 & 0.378\end{array}$ & $\begin{array}{l}=.136 \\
=: 165 \\
=: 065 \\
=: 153 \\
=.072\end{array}$ & $\begin{array}{l}\mathrm{C} \\
\mathrm{C} \\
\mathrm{C} \\
\mathrm{C} \\
\mathrm{C}\end{array}$ & $\begin{array}{rrr}+20 & 1 & 30.74 \\
+20 & 2 & 34.33 \\
+20 & 2 & 55.90 \\
+20 . & 6 & 40.67 \\
+20 & 7 & 0.50\end{array}$ & $\begin{array}{l}-.16 \\
=.37 \\
+.10 \\
-.33 \\
-.10\end{array}$ \\
\hline $\begin{array}{r}17.4 \\
18.4 \\
20.4 \\
1916\end{array}$ & $\begin{array}{l}\mathrm{Sr} \\
\mathrm{P} \\
\mathrm{Sr}\end{array}$ & & $\begin{array}{l}\mathrm{C} \\
\mathrm{C} \\
\mathrm{C}\end{array}$ & $\begin{array}{lll}8 & 1 & 17.233 \\
8 & 1 & 11.517 \\
8 & 0 & 59.966\end{array}$ & $\begin{array}{l}=.207 \\
=.073 \\
=.164\end{array}$ & $\begin{array}{l}\mathrm{C} \\
\mathrm{C} \\
\mathrm{C}\end{array}$ & $\begin{array}{rrr}+20 & 9 & 12.55 \\
+20 & 9 & 29.75 \\
+20 & 10 & 5.71\end{array}$ & $\begin{array}{l}=.05 \\
=.85 \\
-.19\end{array}$ \\
\hline $\begin{array}{ll}\text { Jan. } & 2.6\end{array}$ & $\mathrm{~s}$ & & $\mathrm{C}$ & $\begin{array}{lll}8 & 15 & 59.096\end{array}$ & -.124 & C & +192752.36 & -.14 \\
\hline $\begin{array}{r}3.6 \\
7.5 \\
8.5 \\
18.5 \\
23.5\end{array}$ & $\begin{array}{l}\mathrm{P} \\
\mathrm{S} \\
\mathrm{Sr} \\
\mathrm{M} \\
\mathrm{Sr}\end{array}$ & $\mathrm{E}$ & $\begin{array}{l}\mathrm{C} \\
\mathrm{C} \\
\mathrm{C} \\
\mathrm{C} \\
\mathrm{C}\end{array}$ & $\begin{array}{lll}8 & 15 & 52.704 \\
8 & 15 & 26.257 \\
8 & 15 & 19.597 \\
8 & 14 & 10.884 \\
8 & 13 & 35.993\end{array}$ & $\begin{array}{l}=.046 \\
=.133 \\
=.083 \\
=.116 \\
-.087\end{array}$ & $\begin{array}{l}\mathrm{C} \\
\mathrm{C} \\
\mathrm{C} \\
\mathrm{C} \\
\mathbf{C}\end{array}$ & $\begin{array}{rrr}+19 & 28 & 13.64 \\
+19 & 29 & 40.50 \\
+19 & 30 & 3.05 \\
+19 & 33 & 48.89 \\
+19 & 35 & 42.83\end{array}$ & $\begin{array}{l}-.26 \\
-.40 \\
+.05 \\
+.19 \\
-.37\end{array}$ \\
\hline 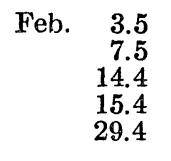 & $\begin{array}{l}\mathrm{S} \\
\mathrm{S} \\
\mathrm{P} \\
\mathrm{Sr} \\
\mathrm{P}\end{array}$ & W & $\begin{array}{l}\mathrm{C} \\
\mathrm{C} \\
\mathrm{C} \\
\mathrm{C} \\
\mathrm{C}\end{array}$ & $\begin{array}{rrr}8 & 12 & 19.898 \\
8 & 11 & 53.111 \\
8 & 11 & 8.089 \\
8 & 11 & 1.933 \\
8 & 9 & 43.053\end{array}$ & $\begin{array}{l}=.162 \\
=: 139 \\
=: 121 \\
=: 077 \\
.127\end{array}$ & $\begin{array}{l}\mathrm{C} \\
\mathbf{C} \\
\mathbf{C} \\
\mathbf{C} \\
\mathbf{C}\end{array}$ & $\begin{array}{r}+193951.65 \\
+1941 \\
+19.62 \\
+19434.19 \\
+19447.04 \\
+194825.54\end{array}$ & $\begin{array}{l}-.35 \\
+.02 \\
+.29 \\
-.16 \\
-.56\end{array}$ \\
\hline $\begin{array}{lr}\text { Mar. } & 4.4 \\
\text { Dec. } & 17.6 \\
& 22.6 \\
& 25.6\end{array}$ & $\begin{array}{l}\mathrm{Sr} \\
\mathrm{M} \\
\mathrm{S} \\
\mathrm{Sr}\end{array}$ & $\begin{array}{l}\mathrm{W} \\
\mathrm{E} \\
\mathrm{E}\end{array}$ & $\begin{array}{l}\mathrm{C} \\
\mathrm{C} \\
\mathrm{C} \\
\mathrm{C}\end{array}$ & $\begin{array}{rrr}8 & 9 & 23.769 \\
8 & 26 & 53.138 \\
8 & 26 & 26.559 \\
8 & 26 & 9.668\end{array}$ & $\begin{array}{l}=.101 \\
=.092 \\
=.141 \\
=.082\end{array}$ & $\begin{array}{l}\text { C } \\
\mathrm{C} \\
\mathrm{C} \\
\mathrm{C}\end{array}$ & $\begin{array}{rrr}+19 & 49 & 29.72 \\
+18 & 55 & 29.02 \\
+18 & 297 \\
+18 & 58 & 2.56\end{array}$ & $\begin{array}{l}-.28 \\
\pm .32 \\
\pm .21 \\
-.14\end{array}$ \\
\hline \begin{tabular}{rr}
\multicolumn{2}{c}{1917} \\
Jan. $\quad 6.6$ \\
7.6 \\
9.5 \\
18.5 \\
25.5
\end{tabular} & $\begin{array}{l}\mathrm{P} \\
\mathrm{M} \\
\mathrm{P} \\
\mathrm{P} \\
\mathrm{S}\end{array}$ & W & $\begin{array}{l}\mathrm{C} \\
\mathrm{C} \\
\mathrm{C} \\
\mathrm{C} \\
\mathrm{C}\end{array}$ & $\begin{array}{lll}8 & 24 & 55.487 \\
8 & 24 & 48.909 \\
8 & 24 & 35.700 \\
8 & 23 & 34.432 \\
8 & 22 & 45.941\end{array}$ & $\begin{array}{l}=.103 \\
=: 131 \\
=: 110 \\
=: 128 \\
=.129\end{array}$ & $\begin{array}{l}\mathrm{C} \\
\mathrm{C} \\
\mathrm{C} \\
\mathrm{C} \\
\mathrm{C}\end{array}$ & $\begin{array}{rrr}+19 & 2 & 25.03 \\
+19 & 2 & 47.22 \\
+19 & 3 & 33.61 \\
+19 & 7 & 7.94 \\
+19 & 9 & 58.08\end{array}$ & $\begin{array}{l}+.93 \\
+.12 \\
+.01 \\
+.06 \\
+.58\end{array}$ \\
\hline $\begin{array}{r}26.5 \\
28.5 \\
30.5 \\
\text { Feb. } 7.5 \\
14.4\end{array}$ & $\begin{array}{l}\mathrm{Sr} \\
\mathrm{M} \\
\mathrm{Sr} \\
\mathrm{Sr} \\
\mathrm{P}\end{array}$ & W & $\begin{array}{l}\mathrm{C} \\
\mathrm{C} \\
\mathrm{C} \\
\mathrm{C} \\
\mathrm{C}\end{array}$ & $\begin{array}{lll}8 & 22 & 39.034 \\
8 & 22 & 25.149 \\
8 & 22 & 11.393 \\
8 & 21 & 17.219 \\
8 & 20 & 31.929\end{array}$ & $\begin{array}{l}=.096 \\
=: 121 \\
=.097 \\
=.141 \\
=.171\end{array}$ & $\begin{array}{l}\mathrm{C} \\
\mathrm{C} \\
\mathrm{C} \\
\mathrm{C} \\
\mathrm{C}\end{array}$ & $\begin{array}{rrr}+19 & 10 & 22.50 \\
+19 & 11 & 10.47 \\
+19 & 11 & 57.97 \\
+19 & 15 & 6.02 \\
+19 & 17 & 43.74\end{array}$ & $\begin{array}{l}+.80 \\
+.47 \\
-.03 \\
+.18 \\
+.14\end{array}$ \\
\hline
\end{tabular}




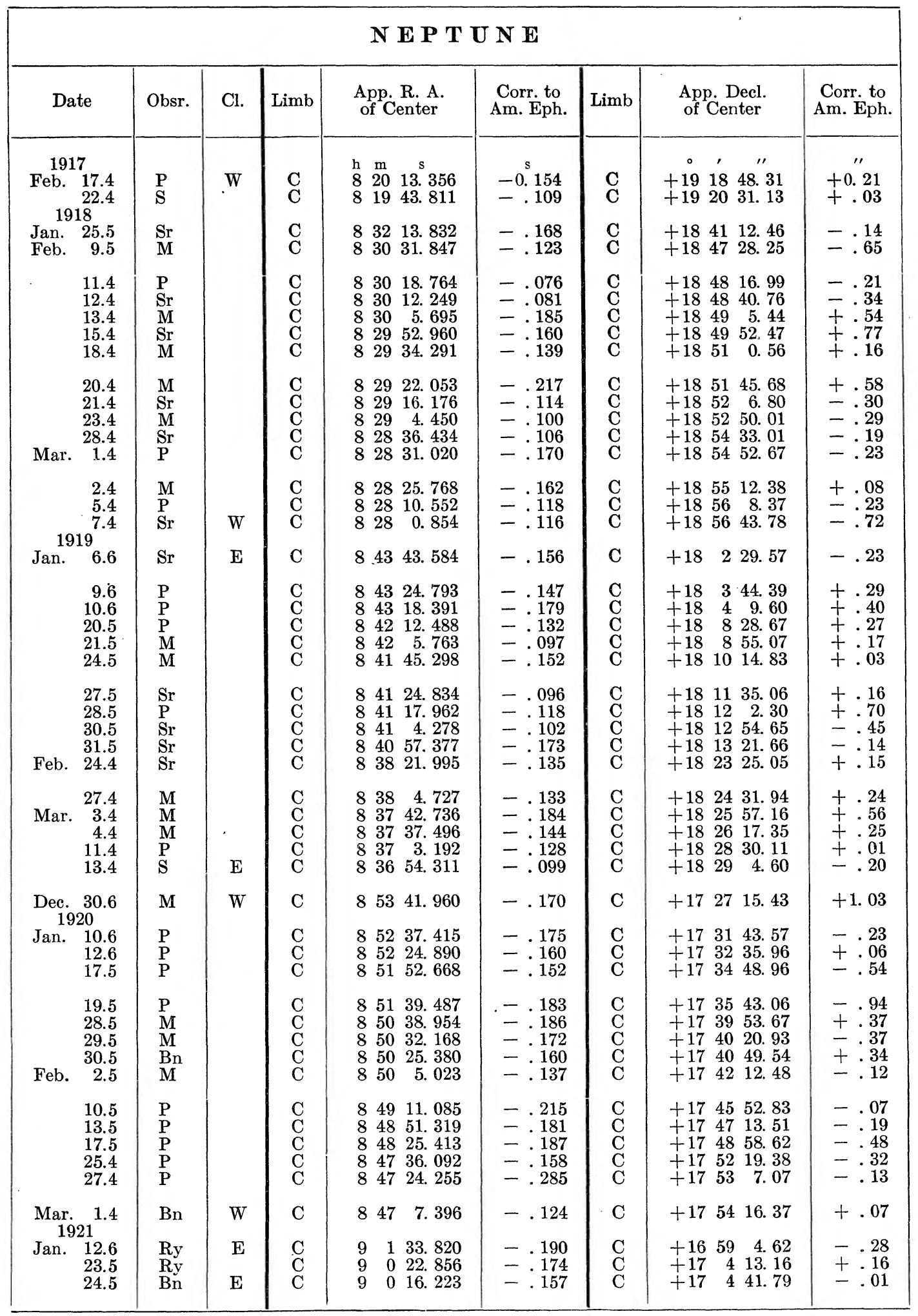




\begin{tabular}{|c|c|c|c|c|c|c|c|c|}
\hline \multicolumn{9}{|c|}{$\mathbf{N} \mathbf{E} \mathbf{T} \mathbf{U} \mathbf{E}$} \\
\hline Date & Obsr. & $\mathrm{Cl}$. & Limb & $\begin{array}{l}\text { App. R. A. } \\
\text { of Center }\end{array}$ & $\begin{array}{l}\text { Corr. to } \\
\text { Am. Eph. }\end{array}$ & $\operatorname{Limb}$ & $\begin{array}{l}\text { App. Decl. } \\
\text { of Center }\end{array}$ & $\begin{array}{l}\text { Corr. to } \\
\text { Am. Eph. }\end{array}$ \\
\hline \begin{tabular}{lr}
\multicolumn{2}{c}{1921} \\
Jan. & 27.5 \\
Feb. & 4.5 \\
& 5.5 \\
6.5 \\
\\
11.5
\end{tabular} & $\begin{array}{l}\mathrm{M} \\
\mathrm{Bn} \\
\mathrm{P} \\
\mathrm{Ry} \\
\mathrm{P}\end{array}$ & $\mathrm{E}$ & $\begin{array}{l}\mathrm{C} \\
\mathrm{C} \\
\mathrm{C}\end{array}$ & $\begin{array}{llr}\mathrm{h} & \mathrm{m} & \mathrm{s} \\
8 & 59 & 56.181 \\
8 & 59 & 2.210 \\
8 & 58 & 55.463 \\
8 & 59 & \\
8 & 58 & 15.300\end{array}$ & $\begin{aligned} & \mathrm{s} \\
&-0.129 \\
&=.140 \\
&=.147 \\
&-.210\end{aligned}$ & $\begin{array}{l}\mathrm{C} \\
\mathrm{C} \\
\mathrm{C} \\
\mathrm{C} \\
\mathrm{C}\end{array}$ & \begin{tabular}{rrr}
0 & \multicolumn{1}{r}{} \\
+17 & 6 & 8.15 \\
+17 & 10 & 0.17 \\
+17 & 10 & 29.21 \\
+17 & 10 & 58.95 \\
+17 & 13 & 21.61
\end{tabular} & $\begin{array}{l}\quad l \prime \\
-0.25 \\
=.33 \\
=.29 \\
+.65 \\
+.41\end{array}$ \\
\hline $\begin{array}{l}12.5 \\
15.5 \\
16.5 \\
24.4 \\
25.4\end{array}$ & $\begin{array}{l}\mathrm{Bn} \\
\mathrm{P} \\
\mathrm{Bn} \\
\mathrm{Ry} \\
\mathrm{P}\end{array}$ & & $\begin{array}{l}\mathrm{C} \\
\mathrm{C} \\
\mathrm{C} \\
\mathrm{C}\end{array}$ & $\begin{array}{rrr}8 & 58 & 8.736 \\
8 & 57 & 49.079 \\
8 & 57 & 42.603 \\
8 & 56 & 52.623 \\
8 & 56 & \end{array}$ & $\begin{array}{l}-.164 \\
=.211 \\
=.217 \\
=.177\end{array}$ & $\begin{array}{l}\mathrm{C} \\
\mathrm{C} \\
\mathrm{C} \\
\mathrm{C}\end{array}$ & 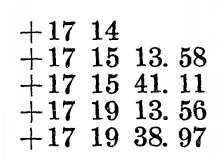 & $\begin{array}{l}+.58 \\
+.51 \\
+.06 \\
-.03\end{array}$ \\
\hline $\begin{array}{r}\text { Mar. } 1.4 \\
3.4 \\
7.4\end{array}$ & $\begin{array}{l}\mathrm{P} \\
\mathrm{Bn} \\
\mathrm{P}\end{array}$ & $\mathrm{E}$ & $\begin{array}{l}\mathrm{C} \\
\mathrm{C} \\
\mathrm{C}\end{array}$ & $\begin{array}{lll}8 & 56 & 23.178 \\
8 & 56 & 11.991 \\
8 & 55 & 50.301\end{array}$ & $\begin{array}{l}-.212 \\
=.119 \\
-.189\end{array}$ & $\begin{array}{l}\mathrm{C} \\
\mathrm{C} \\
\mathrm{C}\end{array}$ & $\begin{array}{rrr}+17 & 21 & 19.21 \\
+17 & 22 & 6.95 \\
+17 & 23 & 38.67\end{array}$ & $\begin{array}{l}+.81 \\
+.75 \\
+.87\end{array}$ \\
\hline Jan. 14.6 & $\mathrm{M}$ & $\mathrm{W}$ & $\mathrm{C}$ & $9 \quad 1032.571$ & -.159 & $\mathrm{C}$ & +162433.95 & -.35 \\
\hline $\begin{array}{l}23.5 \\
24.5 \\
25.5 \\
26.5 \\
30.5\end{array}$ & $\begin{array}{l}\mathrm{Bn} \\
\mathrm{P} \\
\mathrm{Bn} \\
\mathrm{M} \\
\mathrm{Ry}\end{array}$ & & $\begin{array}{l}\text { C } \\
\mathbf{C} \\
\mathbf{C} \\
\mathbf{C} \\
\mathbf{C}\end{array}$ & $\begin{array}{lll}9 & 9 & 35.377 \\
9 & 9 & 28.844 \\
9 & 9 & 22.272 \\
9 & 9 & 15.698 \\
9 & 8 & 49.136\end{array}$ & $\begin{array}{l}=.183 \\
=.176 \\
=.168 \\
=.132 \\
=.094\end{array}$ & $\begin{array}{l}\mathrm{C} \\
\mathrm{C} \\
\mathrm{C} \\
\mathrm{C} \\
\mathrm{C}\end{array}$ & 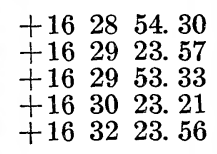 & $\begin{array}{l}+.30 \\
\pm .03 \\
+.03 \\
+.11 \\
+.56\end{array}$ \\
\hline $\begin{array}{cr} & 31.5 \\
\text { Feb. } & 24.5 \\
& 28.4 \\
\text { Mar. } & 5.4 \\
& 8.4\end{array}$ & $\begin{array}{l}\mathrm{P} \\
\mathrm{P} \\
\mathrm{P} \\
\mathrm{Ry} \\
\mathrm{Ry}\end{array}$ & & $\begin{array}{l}\mathrm{C} \\
\mathrm{C} \\
\mathrm{C} \\
\mathrm{C} \\
\mathrm{C}\end{array}$ & $\begin{array}{rrr}9 & 8 & 42.324 \\
9 & 6 & 5.352 \\
9 & 5 & 41.209 \\
9 & 5 & 12.484 \\
9 & 4 & 56.191\end{array}$ & $\begin{array}{l}=.216 \\
=.158 \\
=.191 \\
=.226 \\
=.189\end{array}$ & $\begin{array}{l}\mathrm{C} \\
\mathrm{C} \\
\mathrm{C} \\
\mathrm{C} \\
\mathrm{C}\end{array}$ & 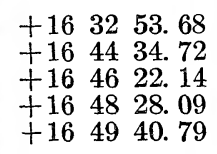 & $\begin{array}{l}+.58 \\
+.32 \\
+.94 \\
+.09 \\
+.69\end{array}$ \\
\hline $\begin{array}{l}12.4 \\
13.4 \\
15.4 \\
16.4 \\
17.4\end{array}$ & $\begin{array}{l}\mathrm{Ry} \\
\mathrm{M} \\
\mathrm{Bn} \\
\mathrm{Bn} \\
\mathrm{M}\end{array}$ & & $\begin{array}{l}\mathrm{C} \\
\mathrm{C} \\
\mathrm{C} \\
\mathrm{C}\end{array}$ & $\begin{array}{lll}9 & 4 & 35.584 \\
9 & 4 & \\
9 & 4 & 20.975 \\
9 & 4 & 16.420 \\
9 & 4 & 11.819\end{array}$ & $\begin{array}{l}-.186 \\
-.265 \\
=.160 \\
-.201\end{array}$ & $\begin{array}{l}\mathrm{C} \\
\mathrm{C} \\
\mathrm{C} \\
\mathrm{C} \\
\mathrm{C}\end{array}$ & 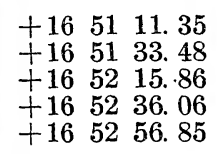 & $\begin{array}{l}+.25 \\
+.58 \\
+.76 \\
+.46 \\
+1.05\end{array}$ \\
\hline \begin{tabular}{lr}
\multicolumn{2}{c}{1923} \\
Jan. & 9.6 \\
& 30.5 \\
Feb. & 7.5 \\
& 8.5
\end{tabular} & $\begin{array}{l}\mathrm{P} \\
\mathrm{Ry} \\
\mathrm{Ry} \\
\mathrm{Ry}\end{array}$ & & $\begin{array}{l}\mathrm{C} \\
\mathrm{C} \\
\mathrm{C} \\
\mathrm{C}\end{array}$ & $\begin{array}{rrr}9 & 20 & 8.294 \\
9 & 17 & 57.910 \\
9 & 17 & 4.760 \\
9 & 16 & 58.078\end{array}$ & $\begin{array}{l}=.186 \\
=.160 \\
=.180 \\
=.202\end{array}$ & $\begin{array}{l}\mathrm{C} \\
\mathrm{C} \\
\mathrm{C} \\
\mathrm{C}\end{array}$ & $\begin{array}{r}+154527.40 \\
+155544.84 \\
+155954.26 \\
+16 \quad 0 \quad 24.86\end{array}$ & $\begin{array}{l}+.80 \\
+.24 \\
+.76 \\
+.36\end{array}$ \\
\hline $\begin{array}{l}11.5 \\
16.5 \\
19.5 \\
20.5 \\
21.5\end{array}$ & $\begin{array}{l}\text { Ry } \\
P \\
P \\
P \\
\text { Ry }\end{array}$ & & $\begin{array}{l}\mathrm{C} \\
\mathrm{C} \\
\mathrm{C} \\
\mathrm{C} \\
\mathrm{C}\end{array}$ & $\begin{array}{rrr}9 & 16 & 38.139 \\
9 & 16 & 5.251 \\
9 & 15 & 45.796 \\
9 & 15 & 39.403 \\
9 & 15 & 32.960\end{array}$ & $\begin{array}{l}=.201 \\
=.179 \\
=.184 \\
=.167 \\
=.240\end{array}$ & $\begin{array}{l}\mathrm{C} \\
\mathrm{C} \\
\mathrm{C} \\
\mathrm{C} \\
\mathrm{C}\end{array}$ & $\begin{array}{rrr}+16 & 1 & 57.64 \\
+16 & 4 & 31.12 \\
+16 & 6 & 0.52 \\
+16 & 6 & 30.52 \\
+16 & 7 & 0.31\end{array}$ & $\begin{array}{l}+.24 \\
+.72 \\
+.02 \\
+.32 \\
+.61\end{array}$ \\
\hline $\begin{array}{r}23.5 \\
\text { Mar. } 1.4 \\
2.4 \\
4.4 \\
5.4\end{array}$ & $\begin{array}{l}\text { Ry } \\
B n \\
B n \\
\text { Ry } \\
P\end{array}$ & & $\begin{array}{l}\mathrm{C} \\
\mathrm{C} \\
\mathrm{C} \\
\mathrm{C} \\
\mathrm{C}\end{array}$ & $\begin{array}{lll}9 & 15 & 20.316 \\
9 & 14 & 43.689 \\
9 & 14 & 37.794 \\
9 & 14 & 26.067 \\
9 & 14 & 20.357\end{array}$ & $\begin{array}{l}-.244 \\
=.171 \\
=.146 \\
=.223 \\
=.193\end{array}$ & $\begin{array}{l}\mathrm{C} \\
\mathrm{C} \\
\mathrm{C} \\
\mathrm{C} \\
\mathrm{C}\end{array}$ & $\begin{array}{rrr}+16 & 7 & 58.59 \\
+16 & 10 & 47.07 \\
+16 & 11 & 14.50 \\
+16 & 12 & 8.85 \\
+16 & 12 & 35.07\end{array}$ & $\begin{array}{l}+.49 \\
\pm .33 \\
+.10 \\
+.55 \\
+.47\end{array}$ \\
\hline$\underset{1924}{\operatorname{Mar} .4}$ & Ry & & $\mathrm{C}$ & 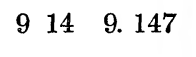 & -.143 & $\mathrm{C}$ & +161326.93 & +.63 \\
\hline $\begin{array}{rr}\text { Jan. } & 14.6 \\
18.6 \\
23.6\end{array}$ & $\begin{array}{l}\text { Bn } \\
\text { Ry } \\
\text { Ry }\end{array}$ & & $\begin{array}{l}\mathrm{C} \\
\mathrm{C} \\
\mathrm{C}\end{array}$ & $\begin{array}{lll}9 & 28 & 41.381 \\
9 & 28 & 17.799 \\
9 & 27 & 47.178\end{array}$ & $\begin{array}{l}=.219 \\
=.211 \\
=.132\end{array}$ & $\begin{array}{l}\mathrm{C} \\
\mathrm{C} \\
\mathrm{C}\end{array}$ & $\begin{array}{rrr}+15 & 9 & 38.25 \\
+15 & 11 & 34.42 \\
+15 & 14 & 5.72\end{array}$ & $\begin{array}{l}+1.05 \\
+.42 \\
+.32\end{array}$ \\
\hline $\begin{array}{cr}\text { Feb. } & 8.5 \\
13.5 \\
16.5 \\
\\
27.5 \\
\text { Mar. } 3.4\end{array}$ & $\begin{array}{l}\mathrm{Bn} \\
\mathrm{Bn} \\
\mathrm{P} \\
\mathrm{Ry} \\
\mathrm{Ry}\end{array}$ & W & $\begin{array}{l}\mathrm{C} \\
\mathrm{C} \\
\mathrm{C} \\
\mathrm{C} \\
\mathrm{C}\end{array}$ & $\begin{array}{rrr}9 & 26 & 3.148 \\
9 & 25 & 30.125 \\
9 & 25 & 10.397 \\
9 & 24 & 0.349 \\
9 & 23 & 30.210\end{array}$ & $\begin{array}{l}=.202 \\
=.185 \\
=.233 \\
=.181 \\
=.180\end{array}$ & $\begin{array}{l}\mathrm{C} \\
\mathrm{C} \\
\mathrm{C} \\
\mathrm{C} \\
\mathrm{C}\end{array}$ & 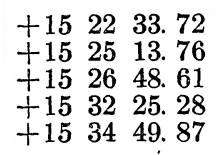 & $\begin{array}{l}+.72 \\
+.66 \\
+.61 \\
+.38 \\
+.97\end{array}$ \\
\hline
\end{tabular}




\begin{tabular}{|c|c|c|c|c|c|c|c|c|}
\hline \multicolumn{9}{|c|}{$\mathbf{N} \mathbf{E} \mathbf{T} \mathbf{U} \mathbf{E}$} \\
\hline Date & Obsr. & $\mathrm{Cl}$. & Limb & $\begin{array}{l}\text { App. R. A. } \\
\text { of Center }\end{array}$ & $\begin{array}{l}\text { Corr. to } \\
\text { Am. Eph. }\end{array}$ & Limb & $\begin{array}{l}\text { App. Decl. } \\
\text { of Center }\end{array}$ & $\begin{array}{l}\text { Corr. to } \\
\text { Am. Eph. }\end{array}$ \\
\hline \begin{tabular}{rr}
\multicolumn{2}{c}{1924} \\
Mar. 7.4 \\
8.4 \\
13.4 \\
14.4 \\
15.4
\end{tabular} & $\begin{array}{l}\mathrm{P} \\
\mathrm{Ry} \\
\mathrm{Ry} \\
\mathrm{M} \\
\mathrm{Bn}\end{array}$ & W & $\begin{array}{l}\mathrm{C} \\
\mathrm{C} \\
\mathrm{C} \\
\mathrm{C} \\
\mathrm{C}\end{array}$ & \begin{tabular}{llr}
$\mathrm{h}$ & $\mathrm{m}$ & \multicolumn{1}{c}{$\mathrm{s}$} \\
9 & 23 & 7.208 \\
9 & 23 & 1.571 \\
9 & 22 & 34.738 \\
9 & 22 & 29.590 \\
9 & 22 & 24.588
\end{tabular} & $\begin{array}{r}\mathrm{s} \\
-0.152 \\
-.199 \\
-.212 \\
-.230 \\
-.192\end{array}$ & $\begin{array}{l}\mathrm{C} \\
\mathrm{C} \\
\mathrm{C} \\
\mathrm{C} \\
\mathrm{C}\end{array}$ & \begin{tabular}{rrr}
$\circ$ & $\prime$ & \multicolumn{1}{c}{} \\
+15 & 36 & 39.21 \\
+15 & 37 & 6.00 \\
+15 & 39 & 13.99 \\
+15 & 39 & 37.39 \\
+15 & 40 & 1.55
\end{tabular} & $\begin{array}{l}1 " \\
+0.51 \\
+.70 \\
+1.19 \\
+.29 \\
+.55\end{array}$ \\
\hline \begin{tabular}{cc}
\multicolumn{2}{c}{1925} \\
Jan. 7.1 \\
15.1 \\
21.1 \\
31.0
\end{tabular} & $\begin{array}{l}\mathrm{P} \\
\mathrm{Bn} \\
\mathrm{P} \\
\mathrm{Ry}\end{array}$ & & $\begin{array}{l}\mathrm{C} \\
\mathrm{C} \\
\mathrm{C} \\
\mathrm{C}\end{array}$ & $\begin{array}{lll}9 & 38 & 16.198 \\
9 & 37 & 33.497 \\
9 & 36 & 58.516 \\
9 & 35 & 56.149\end{array}$ & $\begin{array}{l}-.162 \\
-.163 \\
-.174 \\
-.171\end{array}$ & $\begin{array}{l}\mathrm{C} \\
\mathrm{C} \\
\mathrm{C} \\
\mathrm{C}\end{array}$ & $\begin{array}{rrr}+14 & 27 & 2.75 \\
+14 & 30 & 44.49 \\
+1433 & 43.14 \\
+1439 & 0.70\end{array}$ & $\begin{array}{r}+.45 \\
+1.09 \\
+.14 \\
.00\end{array}$ \\
\hline $\begin{array}{r}\text { Feb. } \quad 1.0 \\
5.0 \\
8.0 \\
9.0 \\
13.0\end{array}$ & $\begin{array}{l}\mathrm{P} \\
\mathrm{Ry} \\
\mathrm{Ry} \\
\mathrm{P} \\
\mathrm{P}\end{array}$ & & $\begin{array}{l}\mathrm{C} \\
\mathrm{C} \\
\mathrm{C} \\
\mathrm{C} \\
\mathrm{C}\end{array}$ & $\begin{array}{llr}9 & 35 & 49.756 \\
9 & 35 & 23.793 \\
9 & 35 & 4.140 \\
9 & 34 & 57.533 \\
9 & 34 & 31.312\end{array}$ & $\begin{array}{l}-.134 \\
=.137 \\
=.160 \\
=.207 \\
-.158\end{array}$ & $\begin{array}{l}\mathbf{C} \\
\mathbf{C} \\
\mathbf{C} \\
\mathbf{C} \\
\mathbf{C}\end{array}$ & 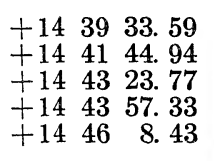 & $\begin{array}{r}+.29 \\
+.44 \\
+.27 \\
+.83 \\
+.13\end{array}$ \\
\hline $\begin{array}{l}19.0 \\
20.0 \\
21.0\end{array}$ & $\begin{array}{l}\mathrm{Bn} \\
\mathrm{Bn} \\
\mathrm{P}\end{array}$ & $W$ & $\begin{array}{l}\mathrm{C} \\
\mathrm{C} \\
\mathrm{C}\end{array}$ & $\begin{array}{llll}9 & 33 & 52.086 \\
9 & 33 & 45 . & 638 \\
9 & 33 & 39 . & 104\end{array}$ & $\begin{array}{l}-.174 \\
=.152 \\
-.236\end{array}$ & $\begin{array}{l}\mathbf{C} \\
\mathbf{C} \\
\mathbf{C}\end{array}$ & $\begin{array}{r}+144925.34 \\
+144957.49 \\
+145029.08\end{array}$ & $\begin{array}{l}+.84 \\
+.69 \\
+.08\end{array}$ \\
\hline \multicolumn{9}{|c|}{$\mathbf{V E S T A} \mathbf{A}^{1}$} \\
\hline \begin{tabular}{rr}
\multicolumn{2}{c}{1916} \\
Jan. & 2.8 \\
3.8 \\
7.8 \\
18.7 \\
23.7
\end{tabular} & $\begin{array}{l}\mathrm{S} \\
\mathrm{P} \\
\mathrm{S} \\
\mathrm{M} \\
\mathrm{Sr}\end{array}$ & $\mathrm{E}$ & & $\begin{array}{lll}13 & 14 & 36.210 \\
13 & 15 & 53.390 \\
13 & 20 & 54.635 \\
13 & 33 & 36.998 \\
13 & 38 & 46.832\end{array}$ & & & $\begin{array}{llll}- & 0 & 19 & 29.67 \\
- & 0 & 23 & 54.63 \\
- & 0 & 40 & 19.39 \\
- & 1 & 14 & 43.23 \\
- & 1 & 24 & 48.20\end{array}$ & \\
\hline $\begin{array}{rr}\text { Apr. } & 10.5 \\
12.5 \\
15.5 \\
18.5 \\
\text { May } 10.4\end{array}$ & $\begin{array}{l}\mathrm{M} \\
\mathrm{Sr} \\
\mathrm{S} \\
\mathrm{P} \\
\mathrm{P}\end{array}$ & $\begin{array}{l}\mathrm{W} \\
\mathrm{E}\end{array}$ & & 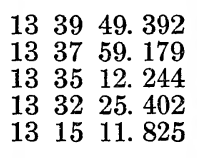 & & & 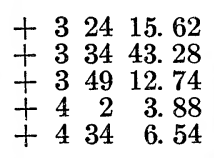 & \\
\hline 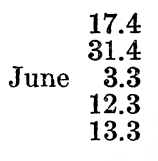 & $\begin{array}{l}\mathbf{S} \\
\mathbf{M} \\
\mathbf{M} \\
\mathrm{Sr} \\
\mathrm{Sr}\end{array}$ & $\mathbf{E}$ & & $\begin{array}{rrr}13 & 11 & 56.681 \\
13 & 9 & 39.305 \\
13 & 9 & 54.666 \\
13 & 12 & 11.998 \\
13 & 12 & 35.371\end{array}$ & & & $\begin{array}{rrr}+ & 19 & 23.61 \\
+ & 17 & 26.16 \\
+2 & 59 & 9.55 \\
+1 & 55 & 37.75 \\
+ & 47 & 50.22\end{array}$ & \\
\hline
\end{tabular}

1 No correction has been applied for parallax to the observed declination of Vesta. 
Sun (Obs. - Eph.)

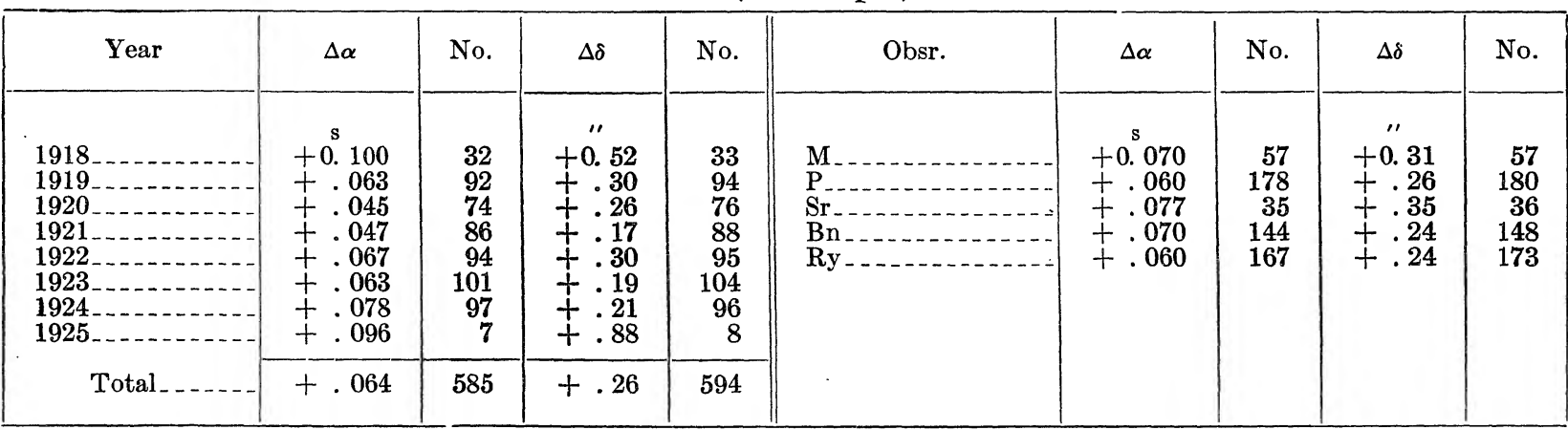

Mercury (Obs.-Eph.)

\begin{tabular}{|c|c|c|c|c|c|c|c|c|c|}
\hline \multirow{2}{*}{ Year } & \multirow{2}{*}{$\Delta \alpha$} & \multirow{2}{*}{ No. } & \multirow{2}{*}{$\Delta \delta$} & \multirow{2}{*}{ No. } & \multirow{2}{*}{ Obsr. } & \multicolumn{3}{|c|}{$\Delta \alpha$} & \multirow{2}{*}{$\Delta \delta$} \\
\hline & & & & & & $\mathrm{C}$ & I & II & \\
\hline $\begin{array}{l}1918- \\
1919- \\
1920 \\
1921 \\
1922 \\
1923 \\
1924\end{array}$ & $\begin{array}{r}\text { s } \\
+0.099 \\
+.083 \\
+.072 \\
+.079 \\
+.094 \\
+.097 \\
+.129\end{array}$ & $\begin{array}{l}14 \\
53 \\
40 \\
37 \\
46 \\
36 \\
40\end{array}$ & \begin{aligned} & \multicolumn{1}{l}{0.37} \\
&$=. .28 \\
&=.23 \\
&=.29 \\
&=.17 \\
&=.04 \\
&=.38\end{aligned}$ & $\begin{array}{l}14 \\
48 \\
39 \\
38 \\
46 \\
36 \\
39\end{array}$ & \multirow{2}{*}{ Total } & $\begin{array}{r}\mathrm{s} \\
+0.049 \\
+.142 \\
+.194 \\
+.085\end{array}$ & $\begin{array}{r}\mathrm{s} \\
+0.018 \\
+.064 \\
+.092 \\
+.116 \\
+.095\end{array}$ & $\begin{array}{r}\mathrm{s} \\
+0.089 \\
+.100 \\
+.048 \\
+.086 \\
+.084\end{array}$ & \begin{aligned} & \multicolumn{1}{l}{} \\
$= & 0.13 \\
= & .45 \\
= & .25 \\
= & .18 \\
- & .10\end{aligned}$ \\
\hline Total_. & +.092 & 266 & -.24 & 260 & & +.108 & +.087 & +.090 & -.25 \\
\hline
\end{tabular}

Venus (Obs.-Eph.)

\begin{tabular}{|c|c|c|c|c|c|c|c|c|c|}
\hline \multirow{2}{*}{ Conj. } & \multirow{2}{*}{$\Delta \alpha$} & \multirow{2}{*}{ No. } & \multirow{2}{*}{$\Delta \delta$} & \multirow{2}{*}{ No. } & \multirow{2}{*}{ Obsr. } & \multicolumn{2}{|c|}{$\Delta \alpha$} & \multicolumn{2}{|c|}{$\Delta \delta$} \\
\hline & & & & & & I & II & $\mathrm{N}$ & $\mathbf{S}$ \\
\hline $\begin{array}{l}1919.7 \\
1921.3 \\
1922.9 \\
1924.5\end{array}$ & $\begin{array}{r}\text { s } \\
+0.013 \\
.019 \\
+.051 \\
+.029\end{array}$ & $\begin{array}{l}29 \\
32 \\
31 \\
30\end{array}$ & $\begin{array}{r}\prime \prime \\
-0.67 \\
-.25 \\
-.00 \\
-.06\end{array}$ & $\begin{array}{l}27 \\
31 \\
30 \\
31\end{array}$ & $\begin{array}{l}\mathrm{M} \\
\mathbf{P} \\
\mathrm{Sr} \\
\mathrm{Bn} \\
\mathrm{Ry}\end{array}$ & $\begin{array}{r}\mathrm{s} \\
+0.038 \\
-.016 \\
=.032 \\
=.012 \\
-.075\end{array}$ & $\begin{array}{r}\text { s } \\
-0.015 \\
+.078 \\
+.003 \\
+.075 \\
+.060\end{array}$ & \begin{aligned} & \multicolumn{1}{c}{} \\
&$= .88 \\
&=.14 \\
&=.96 \\
&=.26 \\
&+.19\end{aligned}$ & $\begin{aligned} & = \\
+ & 0.25 \\
= & .47 \\
= & .52 \\
= & .11 \\
- & .39\end{aligned}$ \\
\hline Total_ & +.019 & 122 & -.24 & 119 & Total & -.031 & +.062 & -.17 & -.31 \\
\hline
\end{tabular}

Moon $(O-C)$

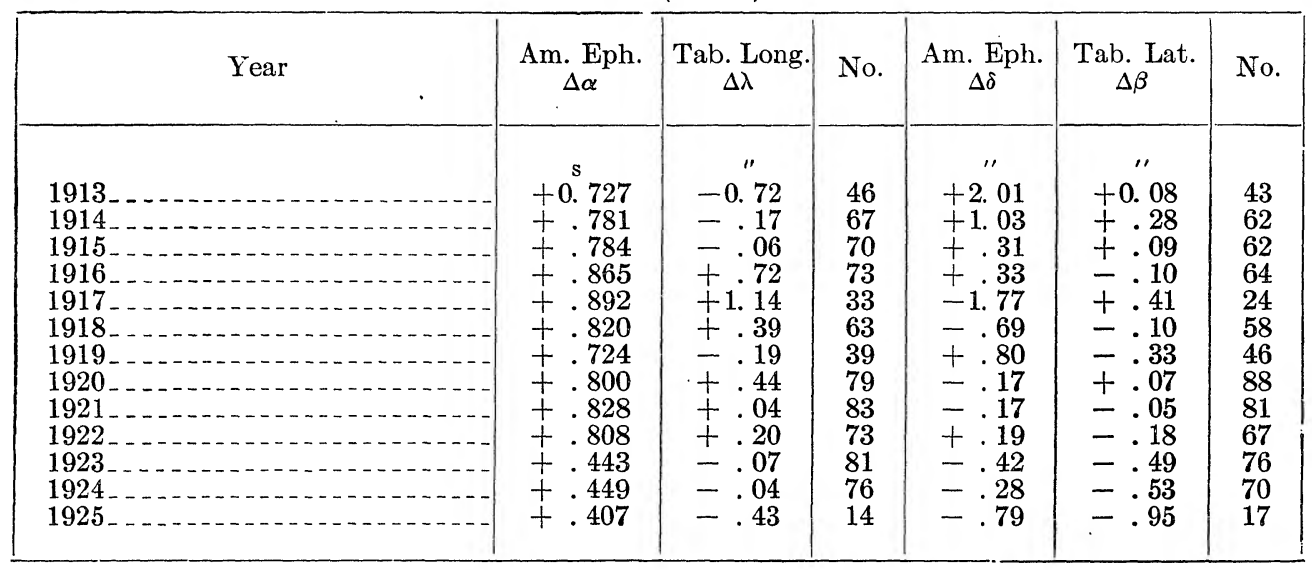


RESULTS OF OBSERVATIONS OF THE SUN, MOON, AND PLANETS

Moon, Longitude, Means by Lunations $(0-C)$

\begin{tabular}{|c|c|c|c|c|c|c|c|c|c|c|c|c|c|}
\hline & January & $\begin{array}{l}\text { Febru- } \\
\text { ary }\end{array}$ & March & April & May & June & July & August & $\underset{\text { Ser }}{\text { Septem- }}$ & October & $\begin{array}{l}\text { Novem- } \\
\text { ber }\end{array}$ & $\begin{array}{c}\text { Decem- } \\
\text { ber }\end{array}$ & Mean \\
\hline $1913 \ldots$ & " & " & " & " & " & " & " & $-1.96_{9}$ & $-0.80_{8}$ & ${ }^{\prime \prime}-70_{10}$ & $-0.05_{10}$ & -0.189 & $-0.72_{46}^{\prime \prime}$ \\
\hline 1914 & $+0.53_{7}$ & $+0.50_{3}$ & $-0.80_{7}$ & $-0.60_{8}$ & $+0.40_{3}$ & $-0.51_{7}$ & $+0.62_{6}$ & & $-0.70_{9}$ & $-0.23_{7}$ & $-0.77_{3}$ & $\left\{\begin{array}{r}+0.42_{4} \\
+0.40_{6}\end{array}\right.$ & $-0.01_{70}$ \\
\hline 1915 & $+1.56_{5}$ & & $\left\{\begin{array}{l}+0.022_{8} \\
-0.37_{9}\end{array}\right.$ & $-0.55_{2}$ & & $-0.11_{8}$ & $-0.32_{5}$ & & $-0.86_{10}$ & $-1.10_{6}$ & $+0.14_{5}$ & $+1.12_{9}$ & $-0.05_{67}$ \\
\hline $\begin{array}{l}1916 \ldots \\
1917 \ldots\end{array}$ & $\begin{array}{l}+0.66_{5} \\
+1.36_{5}\end{array}$ & $\begin{array}{r}+0.88_{4} \\
+1.30_{6}\end{array}$ & $\begin{array}{r}+0.57_{9} \\
+1.45_{4}\end{array}$ & $\begin{array}{l}-0.10_{8} \\
+0.85_{8}\end{array}$ & $\begin{array}{r}+0.70_{3} \\
+1.12_{6}\end{array}$ & $\begin{array}{r}+0.50_{5} \\
+0.92_{4}\end{array}$ & $+0.40_{4}$ & $-0.80_{1}$ & $+1.35_{8}$ & $+1.06_{8}$ & & $+0.84_{8}$ & $\begin{array}{l}+0.71_{73} \\
+1.14_{33}\end{array}$ \\
\hline $\begin{array}{l}1918 \ldots \\
1919 .-\end{array}$ & $\begin{array}{l}+0.16_{5} \\
-0.10_{5}\end{array}$ & $\begin{array}{l}+0.355_{9} \\
-0.07_{3}\end{array}$ & $\begin{array}{l}+1.04_{12} \\
+0.10_{5}\end{array}$ & $\begin{array}{l}+1.04_{5} \\
+1.03_{3}\end{array}$ & $\begin{array}{l}-0.18_{9} \\
-0.10_{1}\end{array}$ & $\begin{array}{l}-0.63_{7} \\
-0.58_{6}\end{array}$ & $-0.70_{1}$ & $-0.74_{5}$ & $-1.00_{2}$ & $\begin{array}{l}+1.06_{7} \\
-0.50_{3}\end{array}$ & $\begin{array}{l}+0.23_{7} \\
+0.50_{3}\end{array}$ & $-0.20_{2}$ & $\begin{array}{l}+0.39_{63} \\
-0.19_{37}\end{array}$ \\
\hline 192 & $+0.46_{5}$ & $+1.28_{5}$ & $+1.55_{4}$ & $+0.50_{8}$ & $+0.07_{6}$ & $\left\{\begin{array}{l}+0.37_{11} \\
+0.28_{6}\end{array}\right.$ & $+0.49_{7}$ & $-1.85_{4}$ & $+0.82_{11}$ & $+0.14_{10}$ & $+0.10_{4}$ & $-0.30_{1}$ & $+0.39_{82}$ \\
\hline $\begin{array}{l}1921 \ldots \\
1922 \ldots\end{array}$ & $\begin{array}{l}+0.27_{6} \\
+0.90_{6}\end{array}$ & $\begin{array}{l}+1.50_{5} \\
+0.62_{5}\end{array}$ & $\begin{array}{l}+0.65_{6} \\
+0.21_{9}\end{array}$ & $-0.12_{6}$ & $\left\{\begin{array}{l}-0.60_{9} \\
+0.04_{8}\end{array}\right.$ & $\begin{array}{l}-0.26_{8} \\
-0.55_{6}\end{array}$ & $\begin{array}{r}-0.34_{8} \\
+0.12\end{array}$ & $\begin{array}{l}-1.13_{10} \\
-1.23_{3}\end{array}$ & $\begin{array}{l}+0.16_{5} \\
-0.10_{8}\end{array}$ & $\begin{array}{r}+0.53_{9} \\
+0.21_{9}\end{array}$ & $\begin{array}{l}+1.18_{4} \\
+0.55_{9}\end{array}$ & $\begin{array}{l}+0.47_{6} \\
+0.75_{6}\end{array}$ & $\begin{array}{l}+0.05_{82} \\
+0.20_{73}\end{array}$ \\
\hline Mean. & +0.64 & +0.80 & +0.44 & +0.26 & +0.22 & -0.06 & +0.16 & -1.38 & -0.14 & +0.05 & +0.31 & +0.40 & \\
\hline $1923 \ldots$ & $\left\{\begin{array}{r}+0.33_{3} \\
+0.43_{3}\end{array}\right.$ & & $\left\{\begin{array}{l}-0.32_{5} \\
+0.03_{6}\end{array}\right.$ & \}$+0.17_{6}$ & $+0.22_{8}$ & $-0.04_{5}$ & $+0.03_{7}$ & $-0.60_{7}$ & $-0.11_{9}$ & $+0.26_{7}$ & $-0.34_{9}$ & $-0.43_{6}$ & $-0.07_{81}$ \\
\hline $\begin{array}{l}1924 \ldots \\
1925 \ldots\end{array}$ & $\begin{array}{l}-0.27_{6} \\
-0.23_{10}\end{array}$ & $\begin{array}{r}-0.20_{3} \\
-0.43_{6}\end{array}$ & $\begin{array}{l}0.37_{7} \\
\end{array}$ & $+0.08_{5}$ & $+0.15_{4}$ & $-0.93_{3}$ & $-0.09_{9}$ & $+0.13_{7}$ & $-0.05_{8}$ & $-0.50_{8}$ & $+0.15_{6}$ & $+0.05_{8}$ & $\begin{array}{l}-0.06_{74} \\
-0.30_{16}\end{array}$ \\
\hline Mean_. & -0.02 & -0.32 & +0.03 & +0.12 & +0.18 & -0.48 & -0.03 & -0.23 & -0.08 & -0.12 & -0.10 & -0.19 & \\
\hline
\end{tabular}

Moon, Latitude, Means by Lunations $(O-C)$

\begin{tabular}{|c|c|c|c|c|c|c|c|c|c|c|c|c|c|}
\hline - & January & $\begin{array}{c}\text { Febru- } \\
\text { ary }\end{array}$ & March & April & May & June & July & August & $\begin{array}{c}\text { Septem- } \\
\text { ber }\end{array}$ & October & $\begin{array}{c}\text { Novem- } \\
\text { ber }\end{array}$ & $\begin{array}{c}\text { Decem- } \\
\text { ber }\end{array}$ & Mean \\
\hline 1913 & " & " & " & " & " & " & " & $\begin{array}{c}\prime \prime \\
+0.37_{7}\end{array}$ & $\begin{array}{l}\prime \prime \\
+0.20_{6}\end{array}$ & $-0.18_{9}$ & $-0.12_{11}^{\prime \prime}$ & $-0.21_{9}$ & $-0.02_{42}$ \\
\hline 1914 & $-0.57_{7}$ & $+1.03_{3}$ & $-0.13_{6}$ & $+0.93_{7}$ & $-0.63_{3}$ & $-0.30_{7}$ & $+0.25_{4}$ & & $-0.01_{7}$ & $+0.61_{7}$ & $+2.00_{3}$ & $\left\{\begin{array}{l}+0.50_{4} \\
+0.31_{7}\end{array}\right.$ & $+0.25_{65}$ \\
\hline 1915 & $+0.22_{4}$ & & $\left\{\begin{array}{l}+0.27_{7} \\
+0.5\end{array}\right.$ & $-0.35_{2}$ & & $-0.57_{7}$ & $-0.77_{4}$ & & $-0.11_{9}$ & $-0.52_{6}$ & $+1.30_{5}$ & $\begin{array}{r}1 \\
+0.42_{8}\end{array}$ & $+0.07_{50}$ \\
\hline $\begin{array}{l}1916 \ldots \\
1917 .-\end{array}$ & $\begin{array}{r}-0.08_{4} \\
+0.42_{4}\end{array}$ & $\begin{array}{l}+0.17_{3} \\
+0.22_{4}\end{array}$ & $\begin{array}{r}+0.01_{7} \\
+0.09_{8} \\
+0.05_{2}\end{array}$ & $\begin{array}{l}+0.17_{7} \\
+0.41_{7}\end{array}$ & $\begin{array}{r}+0.40_{3} \\
+1.08_{5}\end{array}$ & $\begin{array}{l}-0.77_{4} \\
-0.55_{2}\end{array}$ & $-1.77_{3}$ & & $-0.20_{6}$ & $-0.12_{8}$ & $-0.12_{9}$ & $+0.47_{9}$ & $\begin{array}{r}-0.07_{64} \\
+0.41_{24}\end{array}$ \\
\hline $\begin{array}{l}1918= \\
1919\end{array}$ & $\begin{array}{l}-0.10_{5} \\
-0.26_{5}\end{array}$ & $\begin{array}{r}-0.45_{8} \\
-0.12_{4}\end{array}$ & $\begin{array}{r}+0.166_{10} \\
-0.60_{7}\end{array}$ & $\begin{array}{r}-0.82_{4} \\
+0.07_{3}\end{array}$ & $\begin{array}{l}-0.11_{9} \\
-1.70_{2}\end{array}$ & $\begin{array}{r}+0.29_{7} \\
-0.58_{6}\end{array}$ & $-2.00_{1}$ & $-0.26_{7}$ & $-0.60_{2}$ & $\begin{array}{l}-0.03_{6} \\
-0.40_{3}\end{array}$ & $\begin{array}{l}-0.13_{6} \\
+0.57_{4}\end{array}$ & $-0.03_{3}$ & $\begin{array}{l}-0.10_{58} \\
-0.38_{44}\end{array}$ \\
\hline $1920 \ldots$ & $-0.68_{5}$ & $+0.13_{6}$ & $-0.20_{6}$ & $+0.78_{10}$ & $0.00_{10}$ & $\left\{\begin{array}{l}-0.04_{10} \\
-0.22\end{array}\right.$ & $-0.30_{7}$ & $+0.67_{6}$ & $+0.25_{10}$ & $+0.42_{9}$ & $-0.37_{3}$ & & $+0.10_{90}$ \\
\hline $\begin{array}{l}1921 \\
1922 \ldots\end{array}$ & $\begin{array}{l}-0.46_{5} \\
-0.68_{5}\end{array}$ & $\begin{array}{l}-0.50_{5} \\
-0.92_{5}\end{array}$ & $\begin{array}{l}+0.03_{7} \\
-0.61_{8}\end{array}$ & $\begin{array}{l}+0.28_{5} \\
-0.70_{2}\end{array}$ & $\begin{array}{l}-0.16_{8} \\
+0.07_{6}\end{array}$ & $\begin{array}{l}+0.16_{7} \\
+0.08_{4}\end{array}$ & $\begin{array}{l}-0.29_{7} \\
-0.50_{5}\end{array}$ & $\begin{array}{l}+0.04_{11} \\
-0.16_{5}\end{array}$ & $\begin{array}{l}-0.31_{8} \\
-0.52_{6}\end{array}$ & $\begin{array}{l}+0.41_{8} \\
+0.43_{7}\end{array}$ & $\begin{array}{l}+0.32_{5} \\
+0.45_{8}\end{array}$ & $\begin{array}{r}-0.34_{5} \\
+0.18_{6}\end{array}$ & $\begin{array}{l}-0.05_{81} \\
-0.18_{67}\end{array}$ \\
\hline Mean_. & -0.24 & -0.06 & -0.04 & +0.09 & -0.13 & -0.25 & -0.57 & +0.13 & -0.16 & +0.07 & +0.43 & +0.16 & \\
\hline 1923 & $\begin{cases}-3.10_{2} \\
-0.18\end{cases}$ & & $\left\{\begin{array}{l}-0.92_{4} \\
-0.70_{6}\end{array}\right.$ & $-0.40_{6}$ & $-0.37_{6}$ & $-0.53_{3}$ & $-0.32_{5}$ & -0.029 & $-0.28_{8}$ & $-0.67_{10}$ & $-0.71_{7}$ & $-0.05_{6}$ & $-0.49_{76}$ \\
\hline $\begin{array}{l}1924 \\
1925\end{array}$ & $\begin{array}{l}-0.36_{5} \\
-1.37_{7}\end{array}$ & $\begin{array}{l}-0.82_{4} \\
-0.65_{10}\end{array}$ & $-0.89_{7}$ & $+0.16_{5}$ & $-0.03_{3}$ & $-1.20_{1}$ & $-0.16_{8}$ & $-0.60_{7}$ & $-0.70_{6}$ & $-0.54_{11}$ & $-0.75_{6}$ & $-0.77_{7}$ & $\begin{array}{l}-0.53_{70} \\
-0.95_{17}\end{array}$ \\
\hline Mean_. & -0.64 & -0.74 & -0.84 & -0.12 & -0.20 & -0.53 & -0.24 & -0.31 & -0.49 & -0.60 & -0.73 & -0.41 & \\
\hline
\end{tabular}

$137506^{\circ}-33-11$ 
Mars (Obs. - Tab.)

\begin{tabular}{|c|c|c|c|c|c|c|}
\hline Opp. & $\Delta \alpha$ & No. & $\Delta \delta$ & No. & $\alpha$ & $\delta$ \\
\hline $\begin{array}{l}1914.0 \\
1916.1 \\
1918.2 \\
1920.3 \\
1924.4\end{array}$ & $\begin{array}{l}\text { s } \\
-0.022 \\
+.009 \\
+.036 \\
+.027 \\
+.051 \\
-.021\end{array}$ & $\begin{array}{l}20 \\
16 \\
19 \\
20 \\
20 \\
20\end{array}$ & $\begin{aligned} & \prime \prime \\
+ & 0.11 \\
\pm & .11 \\
= & .23 \\
= & .22 \\
= & .32 \\
- & .20\end{aligned}$ & $\begin{array}{l}21 \\
17 \\
20 \\
21 \\
20 \\
20\end{array}$ & $\begin{array}{r}\mathrm{h} \\
7.0 \\
9.5 \\
11.9 \\
14.0 \\
17.1 \\
22.4\end{array}$ & $\begin{array}{l}\circ \\
+26 \\
+19 \\
+5 \\
-11 \\
-25 \\
-17\end{array}$ \\
\hline Total $\ldots$ & +.013 & 115 & -.13 & 119 & & \\
\hline
\end{tabular}

Jupiter (Obs. - Eph.)

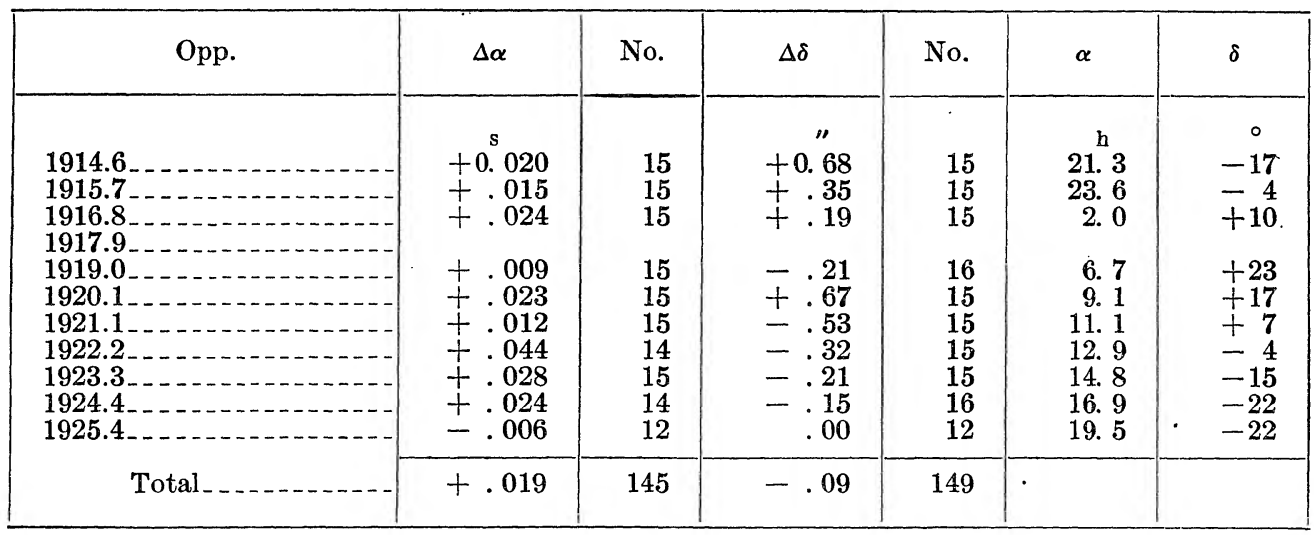

Saturn (Obs.-Eph.)

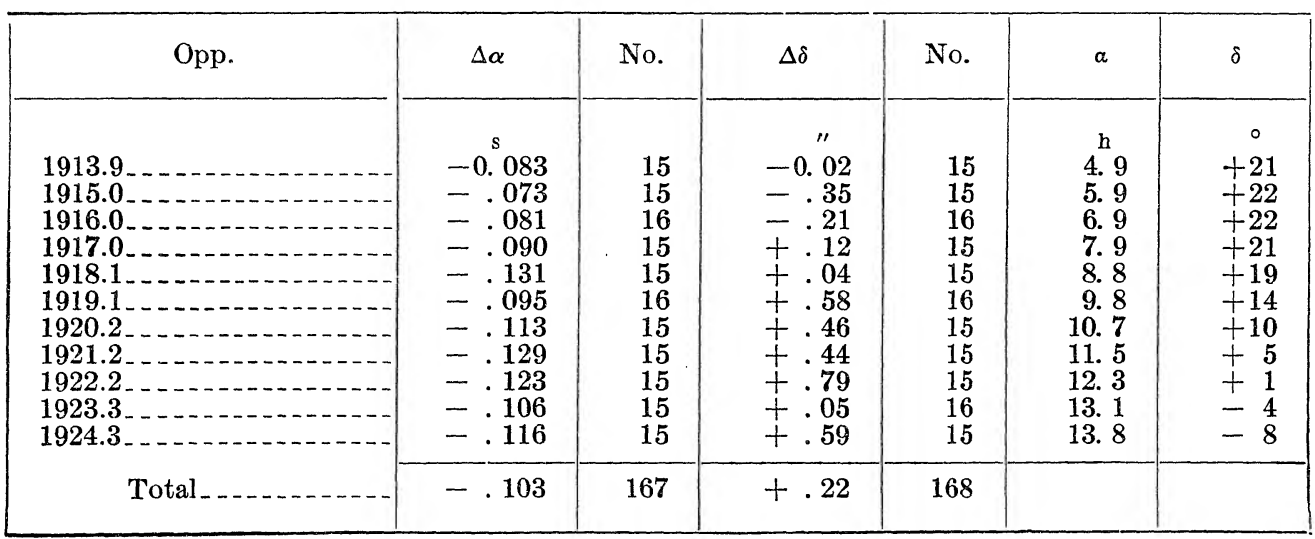


RESULTS OF OBSERVATIONS OF THE SUN, MOON, AND PLANETS

Uranus (Obs. - Eph.)

\begin{tabular}{|c|c|c|c|c|c|c|}
\hline Opp. & $\Delta \alpha$ & No. & $\Delta \delta$ & No. & $\alpha$ & $\delta$ \\
\hline $\begin{array}{l}1914.6 \\
1915.6 \\
1916.6 \\
1918.7 \\
1919.7 \\
1920.7 \\
1921.7\end{array}$ & $\begin{array}{l}\text { s } \\
+0.161 \\
+.134 \\
+.142 \\
\\
+.121 \\
+.120 \\
+.114 \\
+.127 \\
+.131 \\
+.145\end{array}$ & $\begin{array}{l}15 \\
16 \\
15 \\
16 \\
15 \\
15\end{array}$ & $\begin{array}{r} \\
\quad " \\
+0.59 \\
+.07 \\
+.52 \\
\\
+.41 \\
+.69 \\
+1.09 \\
+1.30 \\
+1.22 \\
+1.47\end{array}$ & $\begin{array}{l}16 \\
16 \\
15 \\
17 \\
15 \\
15\end{array}$ & $\begin{array}{l}22.1 \\
22.4 \\
22.6 \\
22.9 \\
23.1 \\
23.4\end{array}$ & 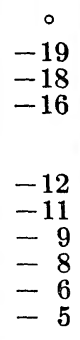 \\
\hline Total & +.133 & 139 & +.82 & 140 & & \\
\hline
\end{tabular}

Neptune (Obs.-Eph.)

\begin{tabular}{|c|c|c|c|c|c|c|}
\hline Opp. & $\Delta \alpha$ & No. & $\Delta \delta$ & No. & $\alpha$ & $\delta$ \\
\hline $\begin{array}{l}1914.1 \\
1915.1 \\
1916.1 \\
1917.1 \\
1918.1 \\
1919.1 \\
1920.1 \\
1921.1 \\
1922.1 \\
1923.1 \\
1924.1 \\
1925.1\end{array}$ & $\begin{array}{l}\mathrm{s} \\
-0.127 \\
-.126 \\
-.110 \\
-.123 \\
-.136 \\
-.136 \\
-.176 \\
-.174 \\
-.180 \\
-.195 \\
-.194 \\
-.169\end{array}$ & $\begin{array}{l}12 \\
13 \\
12 \\
15 \\
15 \\
16 \\
15 \\
14 \\
15 \\
15 \\
13 \\
12\end{array}$ & $\begin{array}{l}{ }^{\prime \prime} \\
-0.16 \\
-.26 \\
-.16 \\
+.20 \\
-.08 \\
+.14 \\
-.10 \\
+.24 \\
+.40 \\
+.39 \\
+.64 \\
+.44\end{array}$ & $\begin{array}{l}12 \\
14 \\
12 \\
15 \\
15 \\
16 \\
15 \\
15 \\
16 \\
15 \\
13 \\
12\end{array}$ & $\begin{array}{l}\text { h } \\
7.9 \\
8.1 \\
8.2 \\
8.4 \\
8.5 \\
8.7 \\
8.8 \\
8.9 \\
9.1 \\
9.3 \\
9.4 \\
9.6\end{array}$ & $\begin{array}{l}\circ \\
+20 \\
+20 \\
+20 \\
+19 \\
+19 \\
+18 \\
+18 \\
+17 \\
+17 \\
+16 \\
+15 \\
+15\end{array}$ \\
\hline Total $\ldots \ldots \ldots$ & -.154 & 167 & +.14 & 170 & & \\
\hline
\end{tabular}

